\title{
Slow Passthrough Around the World: A New Import for Developing Countries?
}

\author{
Jeffrey Frankel, David Parsley and Shang Jin-Wei
}

CID Working Paper No. 116

February 2005

(C) Copyright 2005 Jeffrey Frankel, David Parsley and Shang Jin-Wei and the President and Fellows of Harvard College
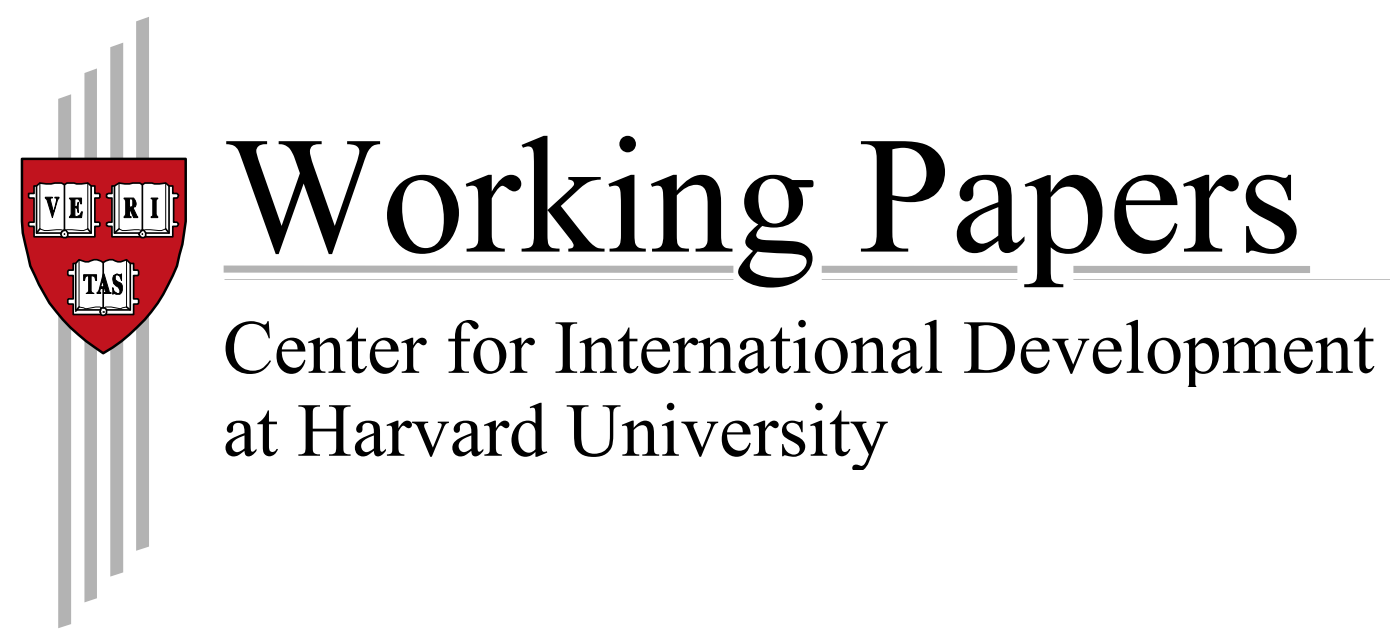


\title{
Slow Passthrough Around the World: A New Import for Developing Countries?
}

\author{
Jeffrey Frankel, David Parsley, and Shang-Jin Wei
}

\begin{abstract}
$\underline{\text { Abstract }}$
Developing countries traditionally experience passthrough of exchange rate changes that is greater and more rapid than high-income countries experience. This is true equally of the determination of prices of imported goods, prices of local competitors' products, and the general CPI. But developing countries in the 1990s experienced a rapid downward trend in the degree of passthrough and speed of adjustment, more so than did high-income countries. As a consequence, slow and incomplete passthrough is no longer exclusively a luxury of industrial countries. Using a new data set -- prices of eight narrowly defined brand commodities, observed in 76 countries -- we find empirical support for some of the factors that have been hypothesized in the literature, but not for others. Significant determinants of the passthrough coefficient include per capita incomes, bilateral distance, tariffs, country size, wages, long-term inflation, and long-term exchange rate variability. Some of these factors changed during the 1990s. Part (and only part) of the downward trend in passthrough to imported goods prices, and in turn to competitors' prices and the CPI, can be explained by changes in the monetary environment including a fall in long-term inflation. Real wages work to reduce passthrough to competitors' prices and the CPI, confirming the hypothesized role of distribution and retail costs in pricing to market. Rising distribution costs, due perhaps to the Balassa-Samuelson-Baumol effect, could contribute to the decline in the passthrough coefficient in some developing countries.
\end{abstract}

The authors would like to thank Charles Engel, Linda Goldberg, and participants at seminars at the IMF and Federal Reserve Board for comments. 


\title{
Slow Passthrough Around the World: A New Import for Developing Countries?
}

\author{
Jeffrey Frankel, David Parsley, and Shang-Jin Wei
}

\section{Introduction}

The phenomenon of slow or incomplete passthrough, which has long been a phenomenon of the market in the United States and other rich countries, increasingly characterizes small developing countries as well. In that sense, slow passthrough was "imported" by lower-income countries in the 1990s. In the aftermath of large devaluations in East Asia, Latin America, and other emerging market countries between December 1994 (Mexico) and December 2001 (Argentina), most observers feared correspondingly large increases in local currency prices. That such price increases did not materialize was a welcome surprise, but was a surprise nonetheless: the conventional wisdom had long been that passthrough is relatively rapid and complete in countries that are small, or less developed, or both. This is why the "small open economy" model has in the past been thought more applicable to them than to rich countries.

The apparent decline in the passthrough coefficient in developing countries in the 1990s has been much discussed informally. Yet it has not been extensively documented, let alone explained. Most of the many econometric studies of passthrough, even those that examine a recent decline in the passthrough coefficient, have focused on prices of imports into industrialized countries, rather than into developing countries. For example, Otani, Shiratsuka, and Shirota (2003) find a decline in passthrough for imports into Japan, which they attribute to increased penetration by intra-firm imports and to a decline in global inflation. ${ }^{1}$ Campa and Goldberg (2001) find a decline in the passthrough coefficient in the 1990s, which they attribute to changing commodity composition more than to a less inflationary environment; but their data set again consists solely of industrialized countries.

A few studies include lower-income countries. Choudhri and Hakura (2001) extend to a sample of 71, including developing countries, the Taylor (2001) and Gagnon and Ihrig (2004) findings that a low-inflation environment reduced passthrough to the CPI in the 1990s. Borensztein and De Gregorio (1999) and Goldfajn and Werlang (2000), study the low passthrough of recent large devaluations in developing countries. ${ }^{2}$ Saiki (2004) includes two developing countries in her study of whether a switch in monetary regime to inflation-targeting is associated with a fall in the passthrough coefficient. .Devereux and Yetman (2002) have 122 countries in their sample. But these are all studies of influences on aggregate price measures,

\footnotetext{
${ }^{1}$ Taylor (2001) proposed that a decline in passthrough of exchange rate changes into the CPI in the 1990s was due to a lower inflationary environment, and looked at US data. Gagnon and Ihrig (2001) extended this claim to a sample of 11 industrialized countries, finding that the standard deviation of inflation explains the coefficient better than does the average inflation rate.

${ }^{2}$ The BIS (2002, p. 92) is among those attributing the low passthrough to the CPI of recent large devaluations in developing countries to a decline in long-run inflation. But Burstein, Eichenbaum and Rebelo (2002) attribute the low observed passthrough in general price indices to the disappearance from consumption of newly expensive import goods, and their replacement in the indices by inferior local substitutes.
} 
the CPI in particular, not on import prices. Few studies concentrate on imports of specific goods into developing countries. ${ }^{3}$ A primary goal of this paper is to extend the literature to a broad sample that includes developing countries, where the question is particularly salient in light of recent experience, and to examine the reported decline in their passthrough coefficients and possible explanations for it.

It is important to be explicit about the degree of disaggregation. "Passed through" to what? We must distinguish between passthrough, on the one hand, in the narrow sense of the determination of prices of goods that are physically imported -- or at least are physically identical with goods that are imported -- versus, on the other hand, the broader sense of the determination of the general price level. There is also an intermediate question: the determination of prices of goods that may be relatively close substitutes for the imported goods but that are produced at home. Gradual passthrough to the general price level has been extensively documented, even for developing countries. The decline in passthrough to the general price level in the 1990s has also been documented. It is primarily to the question of passthrough to narrowly defined import prices that the present paper seeks to contribute. But we will also trace through effects on the prices of domestically produced substitutes and to the general price level, thus facilitating a connection between our findings on passthrough narrowly defined and others' findings on passthrough defined more broadly.

We use data on imports into 76 countries, for eight narrowly defined goods. They are (given with their country of origin): Marlboro Cigarettes (US), Coca-cola (US), Cognac (France), Gilbey's gin (US), Time magazine (US), Kodak Color Film (US), Cointreau Liqueur (France), and Martini \& Rossi Vermouth (Italy). Our data pertain to literally the identical product across different countries. The period is 1990-2001. Further details are given below.

\section{Hypotheses to be tested}

Any theory of incomplete passthrough must posit some barrier to arbitrage between the import good in the country of origin and the same good in the country of purchase. Among the candidate explanations for the barrier to arbitrage are: trade distortions, transportation costs, and the local value added that enters into the distribution process between the dock and the point of retail. ${ }^{4}$ Beyond the question of the arbitrage barriers, some theories model the "pricing to market" phenomenon as a case of optimal price discrimination by firms. ${ }^{5}$ Others, perhaps

\footnotetext{
${ }^{3}$ Aw (1993) examines exports from Taiwan to four countries of footwear, but they are heavily affected by quotas. Also, like Maloney's (1994) study of Chile, the data are for an earlier period. Parsley (2002) examines exports from Hong Kong, and finds little pricing to market.

${ }^{4}$ Among those who emphasize the importance in incomplete passthrough of local distribution costs consisting of nontraded inputs are Burstein, Eichenbaum and Rebelo (2002), Burstein, Neves and Rebelo (2003), Campa and Goldberg (2004), Corsetti and Dedola (2002), Frankel (1984), and many others. Parsley and Wei (2003) offer some detailed evidence that the law of one price holds much better for traded inputs than for the product sold to consumers. They seek to reaffirm the conventional wisdom that the failure of PPP can be partly explained by non-traded goods and services, by refuting the Engel (1999) challenge, that the explanation lies solely in failures of the law of one price among traded goods. Burstein, Eichenbaum, and Rebelo (2003) is another challenge to Engel, this time for four largedevaluation episodes.

${ }^{5}$ In addition to Dornbusch (1987) and Krugman (1987), the price discrimination theory is featured in Marston (1991), Gagnon and Knetter (1995), Yang (1997), Corsetti and Dedola (2002), among many others.
} 
motivated by the notion that the barriers to arbitrage are smaller in the long run than in the short run, model prices as completely sticky in the local currency at a moment in time, but adjusting gradually over time. ${ }^{6}$ Given the diversity of models that have been proposed, it would be good to be able to choose among them empirically or, if it turns out that all raise factors that are important determinants of passthrough, then to get an idea of their relative importance. A second important goal of this paper is to try to see which of the factors that are invoked in the theoretical literature are in practice important and which are not, using a panel of data that extends across a broad set of countries.

There are important connections between the recent experience of developing countries and the various competing models in the theoretical literature. Three testable hypotheses apply (after eliminating the compositional effects that affect aggregate price measures). (1) A priori, if passthrough coefficients are higher in small countries, one could attribute that to a paucity of local substitutes in small countries, as in some models of price discrimination. ${ }^{7}$ (2) If passthrough coefficients are higher (for retail prices) in poor countries, one could attribute that to lower costs for labor and commercial real estate, which are the non-traded inputs into the distribution and retail process. (3) If passthrough coefficients have declined over time, one could attribute that to (a) a less inflationary environment, or (b) to costs of labor and rent that rise over time, as in the Balassa-Samuelson effect (internationally) or Baumol effect (domestically).

The question whether passthrough is indeed lower for developing countries is important for a number of reasons. It matters for the determination of the trade balance, and for whether the small open economy model is appropriate. It also matters for a country's choice of exchange rate regime. It has been observed that developing countries generally are more reluctant to see their exchange rates fluctuate than rich industrial countries are, a phenomenon sometimes labeled "fear of floating." Even among those that have adopted inflation targeting as a monetary framework, it is more common to see them intervene heavily and frequently in the foreign exchange market than developed economies. Several explanations have been proposed for this phenomenon. For the purpose of this paper, we note that a relatively high degree of passthrough for developing countries has been cited as a rationale for the developing countries' stance on exchange rates (e.g., Ho and McCauley, 2003).

One further motivation for this research arises in the recent emphasis that the PPP literature has given to the issue that heterogeneity in parameters can create bias in the estimates from aggregate data. ${ }^{8}$ Allowing parameters to vary by means of fixed effects, across goods or across countries, is one way to address the problem. But a better way is to model the variables

\footnotetext{
${ }^{6}$ Kasa (1992) shows how adjustment costs can generate incomplete passthrough in the short-run. Ghosh and Wolf (1995) study changes in the local price of the Economist magazine in various countries in response to exchange rate changes, and argue that the timing supports the sticky price view, arising from menu costs, better than the pricing to market view, arising from price discrimination. Devereux and Yetman (2002) apply a menu-cost model to the endogenous determination of passthrough. Burstein, Eichenbaum, and Rebelo (2003) includes another sticky-price model.

${ }^{7}$ In the Cournot oligopoly model of Dornbusch (1987), for example, the extent of passthrough is determined by the proportion of foreign firms present in the domestic marketplace, relative to domestic firms.

${ }^{8}$ Imbs, Mumtaz, Ravn, and Rey (2002) claim that problems of aggregation are the source of estimates of apparently slow convergence to PPP. Chen and Engel (2004) reject their argument.
} 
on which the parameters depend. To the extent that variation in passthrough behavior across countries is an issue (especially rich vs. poor countries), and to the extent that such variation is related to differences in income, size, and so forth, our approach may be able to shed more light on the true parameters.

\section{A brief further review of the models in the literature, and their predictions}

A major empirical discovery of recent years is that goods markets are less integrated than previously thought. The Law of One Price fails by surprisingly large margins, even when tests are applied to goods that are narrowly defined into homogeneous categories. An important subset of this research looks at slow or incomplete passthrough of exchange rate changes into import prices: when the exchange rate changes, the price of an imported good does not seem to change by the full amount, at least in the short run.

We have learned steadily about the phenomenon of slow or incomplete passthrough. The subject gets a boost every time there are large increases in a country's exchange rate followed by surprisingly small increases in import prices. For example, some of the early contributions date from the dollar devaluations of the early 1970s. ${ }^{9}$ Next, the large swing in the dollar in the 1980s, unaccompanied by matching swings in import prices, produced a large literature, both theoretical and empirical, on slow or incomplete passthrough. ${ }^{10}$ Krugman (1987) gave it the name Pricing to Market, to indicate that firms were deliberately setting prices in different countries with an eye to their competitors in the local markets. Theoretical models showed how firms should pricediscriminate optimally, as a function of demand elasticities -- for example, Knetter $(1989,1993)$ and Dornbusch (1987). If local demand is highly elastic, foreign firms are forced to absorb exchange rate fluctuations in their profit margins rather than passing them fully through in local markets. More recently, the case of Local Currency Pricing (the price of the importable is unchanged in domestic currency) has been successfully incorporated into modern macroeconomic theory, as a starkly different case from the traditional assumption of Producer Currency Pricing (the change in the exchange rate is fully passed through to the import price). ${ }^{11}$ There is also an extensive empirical literature. ${ }^{12}$

As already noted, any theory of incomplete passthrough must begin with a reason why the law of one price fails, that is, with a barrier to arbitrage. To whatever extent we are talking

\footnotetext{
${ }^{9}$ Kreinen (1977) and Magee (1973).

${ }^{10}$ There are also other reasons why the literature on incomplete passthrough took off in the late 1980s: it provided an application for some tools of game theory that had then been newly imported into international trade theory from industrial organization; it provided an application for new mathematical techniques of option-pricing with continuous-time stochastic processes (e.g., Dixit, Krugman, Baldwin); the micro price data needed for empirical work became available (e.g., Knetter, 1989, 1993); the partial equilibrium exercise of taking exchange rate movements as given became more interesting when models to explain the exchange rate had clearly failed; and slow passthrough into the US market -- particularly in the case of automobiles and other exports from long-horizon Japanese producers (e.g., Marston, 1990; Froot and Klemperer, 1989; Feenstra, 1989; Parsley, 1993; Gagnon and Knetter, 1995; Ohno, 1989) -seemed to help explain the slow reaction of the US trade balance to the 1985-97 depreciation of the dollar (Mann, 1986).

${ }^{11}$ The models of Devereux and Engel (2002) and Devereux, Engel and Storgaard (2002) show how the absence of passthrough reduces the real effects of exchange rate variation.

${ }^{12}$ See Goldberg and Knetter (1997) for a survey.
} 
about goods that are not identical to the foreign good, or perhaps not even close substitutes for it, no further explanation is required. But when we are talking about the identical good, the obvious candidates for barriers to arbitrage fall into two categories: (1) the transport costs, tariffs, and other trade barriers that intervene between the port in the country of export and the port in the country of import, and (2) the costs of distribution and retail that intervene between the dock in the country of import and the customer at the store counter. Many modelers have focused on just one category of barrier or the other, but both are potentially important. We will proxy transport costs with bilateral distance between exporting country and importing country, and will measure trade barriers with data on commodity-specific tariffs. We might expect the effect of distance to be the same for rich and poor importers alike; but poor countries are more likely to have higher trade barriers. We will proxy the costs of distribution and retail by the country's wage rate. These are nontraded services, and so the Baumol and Balassa-Samuelson effects lead us to expect that they may play a smaller role in developing countries than in rich countries. Indeed, the low real cost of retail services in poor countries, as a fraction of the value of the product, should constitute one of the possible explanations for the traditional proposition that passthrough is higher in developing countries.

Whatever their choice for an explanation for the failure of arbitrage, modelers can also be distinguished according to their view of price-setting behavior. Here we distinguish three categories of models: (1) prices are sticky in local currency in the short-run, (2) firms follow rule-of-thumb markup pricing, so that an increase in the exchange rate will be fully passed through to local prices (but in some cases there may be a lag, until previous shipments are sold), ${ }^{13}$ and (3) firms engage in price-discrimination, optimally "pricing to market" so as to take into account the relevant demand elasticities. Price stickiness should show up as slow adjustment, leaving aside the degree of passthrough that holds in the long run. Markup pricing predicts substantial passthrough, but perhaps with a short lag. It seems less straightforward to get at the hypothesis of price discrimination than the other influences. One piece of evidence that might support price discrimination is that small economies tend to experience higher longrun passthrough than large countries, even after conditioning for other determinants like income per capita or wages. The rationale is the Dornbusch (1987) finding from a Cournot oligopoly model: under optimal price discrimination, the passthrough coefficient will be determined by the share of foreign firms in the domestic marketplace, relative to domestic firms (times the degree of competition, defined as the reciprocal of price as a markup over marginal cost). The more heavily foreign firms outnumber domestic firms, the higher the degree of passthrough. In the limit, a very small country under perfect competition experiences complete passthrough. Admittedly there may be an important difference between the size of the presence of foreign versus domestic firms in the domestic market for a particular commodity, and the size of the foreign presence in the economy in the aggregate.

Another piece of evidence in favor of the price discrimination hypothesis would be if passthrough to local prices of imported goods tended to behave similarly to prices of local substitutes. ${ }^{14}$ Under the mark-up pricing hypothesis, by contrast, a devaluation should soon open

\footnotetext{
${ }^{13}$ With full mark-up pricing, passthrough may be complete even though the Law of One Price fails. The two criteria differ. Failure of complete passthrough will invalidate even relative Purchasing Power Parity, while failure of the Law of One Price need invalidate only Absolute PPP.

${ }^{14}$ We would expect passthrough to prices of other local goods to be lower, however. In an optimal-pricing model where imports are intermediate products, Bacchetta and Van Wincoop (2002) show passthrough to consumer goods prices to be low if there is local competition.
} 
up a discrete wedge between the import price and the prices of local competitors. Under the sticky price hypothesis the devaluation should also have that effect, but only gradually over time.

One instance of the size hypothesis is that passthrough is particularly low into the world's largest market, the United States, a proposition that goes back at least to Kreinen (1977). Knetter 1993) and Campa and Goldberg (2002), however, found this to be an artifact of composition, that such apparent differences in passthrough across countries tend to disappear for given industries. So the US dummy is another proposition to be tested.

Another composition hypothesis is the claim of Burstein, Eichenbaum, and Rebelo that recent estimates of low passthrough of devaluations to the CPI are due in part to substitution away from high-end varieties of imports to lower-quality substitutes. If this phenomenon were to constitute the entire explanation for incomplete passthrough, then we would expect that the passthrough would be close to complete and instantaneous for the completely disaggregated import goods in our sample. The ability to discriminate among such hypotheses is one benefit of working with products that are so narrowly defined as to be literally identical in the exporting and importing countries - a pack of Marlboro cigarettes, etc.

We also test two hypotheses regarding a country's longer-term monetary environment. Chronic high rates of inflation affect a country's economic structure, including such institutions as indexation of wages and automatic passthrough of exchange rate changes, as a number of models have shown. We measure the average inflation rate over the preceding five years, to see if it affects the extent of concurrent passthrough and the speed of subsequent adjustment. If so, the lower-inflation environment of the 1990s, relative to the 1970s and 1980s, would clearly be a leading candidate to explain any decline in the passthrough coefficient.

We also test the effect of exchange rate volatility, measured as the standard deviation of monthly exchange rate changes over the preceding five years. At first glance, one might expect exchange rate variability to have the same positive effect on the passthrough coefficient as the long run inflation rate. ${ }^{15}$ The logic here, however, is quite different; it is almost the opposite. Krugman (1989), Froot and Klemperer (1989) and Taylor (2000) hypothesized that a given exchange rate change is less likely to be passed through to import prices in an environment where such fluctuations are common and transitory. Firms fear losing market share, and will wait to see whether the exchange rate change looks permanent before modifying local prices. Thus we expect variability (around the trend) to have a negative effect on the passthrough coefficient, not a positive effect.

There is some empirical documentation in the literature for the familiar claim that exchange rate pass-through tends to be higher in developing economies than in rich countries has been documented. For example, Choudhri and Hakura (2001) reported that for a sample of 12 emerging market economies during 1979-2000, their average one-year pass-through is $26 \%$ (with some individual pass-through degrees as high as 40\%). This is much higher than the average one-year pass-through for a group of non-G3 industrial countries (12\%) or G3 (only $7 \%) .{ }^{16}$ But few have offered explicit explanations for the differential. It may be that low-income countries are on average smaller and more inflation-prone than rich countries. Or it may be that due to

\footnotetext{
${ }^{15}$ Indeed, in the thoery of Devereux and Yetman (2002), exchange rate variability, like price instability, should raise the passthrough coefficient. But they sometimes find the opposite, empirically.

${ }^{16}$ Ho and McCauley (2003).
} 
lower real wages and rents, distribution and retail costs tend to be less important for them. Think of a street vendor in a poor country, as compared to an expensive retail operation in a rich country. (Tariffs and transport costs on the other hand are likely to be more important for developing countries, on average.) It would be useful to know if the difference in passthrough behavior that has been observed historically is a result of these other factors, or pertains to the difference in income per se. Answering this question is another goal of this paper.

\section{Our Approach}

\section{Description of the Data}

The individual goods prices used in this study were compiled by the Economist Intelligence Unit (EIU). The EIU data are collected as part of the Worldwide Cost of Living Survey, and are designed for use by human resource managers in the design of compensation policies. The EIU description is at http://eiu.e-numerate.com/asp/wcol_HelpWhatIsWCOL.asp. The data set contains more than two hundred local currency retail prices of (mostly generic) goods and services collected from 120 cities around the world (some goods are priced at two locations and both prices appear in the data set). The data have been collected annually, during the first week of September since 1990.

We focus on one city (the capital) per country (76 countries), and on well defined, specific products. The EIU product descriptions include the brand name, physical attributes, e.g., size, volume, and in some cases, the type of retail establishment where the price was observed, e.g., supermarket versus mid-priced outlet. Where multiple prices are available for the same product, we selected the supermarket price. Alcoholic beverages are heavily represented in the list: French VSOP Cognac, Gilbey's Gin, Cointreau, and Martini and Rossi Vermouth. This reflects our selection criterion: that the survey must specify the brand that has been priced, rather than anything about the types of products the EIU thinks important for its survey. Most product specifications in the survey are too generic for our criterion, e.g., "facial tissues, box of 100." 17

An additional restriction we imposed on the sample was that the goods be associated with a particular country of origin. We recognize that some of our products might have significant local value added, e.g., Time magazine may be printed locally, Coca-Cola may be locally bottled, and Philip Morris may have Marlboro production facilities outside the United States. Our assumption, however, is that even for these cases the primary content (news articles, CocaCola syrup, Burley tobacco, and/or the recipe) is exported from the U.S. Applying these restrictions yields a sample of 76 cities, eight well-defined goods for the years 1990 to 2001 .

We also collected the prices of local competitor products from the $E I U$ data set. For comparison, Table A lists the imported products, their country of origin, and the domestic substitute good that we use in our analysis. The choice of domestic substitute prices is dictated by the data set and is clearly more precisely matched for some products, e.g., Marlboro cigarettes than others. However, our results are surprisingly robust across products.

\footnotetext{
${ }^{17}$ French VSOP Cognac is an exception to this stringent brand-specific rule; however after checking with the $E I U$ we were told that the Cognac brands were, in fact, specified as Remy Martin, or Courvoisier; however another brand would be surveyed if these were not available, as long as it was VSOP, and not VS, XO, or 3-star. Moreover, our empirical findings also apply to this more general category.
} 
A complete listing of the countries is presented in Table B. For these same countries and years, we use also the nominal exchange rates, hourly labor costs, that are included in the EIU data base.

All of the price series were checked for coding errors. First, price observations (in common currency) that differed from the cross-sectional mean by more than a factor of three were set to missing. Next, price series missing one year's observation were interpolated using the average of the previous and next year's values. Next, we attempted to catch potential coding errors by focusing on within-product/country price swings. Specifically, price changes within a given city of more than $60 \%$, that were subsequently reversed in the next period, were also replaced by the average of the previous and next year's values. Finally, in our reported regressions, we first ran preliminary regressions to identify the largest residual outliers. The observations associated with the top 1 percent of the residuals in these preliminary regressions were then excluded prior to obtaining the estimates reported in the tables.

In addition to the $E I U$ data, we obtained aggregate consumer price indices (used to deflate the nominal wage, and to compute long-term five-year rolling averages of inflation), per capita real GDP, monthly bilateral exchange rate, and aggregate real GDP data from the World Economic Outlook data base. We obtained simple average tariff levels from Table 6.6 of the World Bank publication World Development Indicators 2001. For each country, the tariff data are available for two years - once in the early 1990s and once for the late 1990s. We use the first reported value in our bilateral tariff rate calculations for the years 1990-95 and the most recent tariff rate for the years 1996-2001. Finally, the distance between importer and exporter cities was calculated using the great circle formula using each city's latitude and longitude data obtained from the UN web site www.un.org/Depts/unsd/demog/ctry.htm.

We also obtained matching unit value data from the United Nations Commodity Trade Statistics Database (Comtrade), and matching product-specific tariff data from the UNCTADTRAINS data base. The unit value data were thoroughly checked for errors using the same procedures as for the price data.

\section{The equations estimated}

We begin by estimating an error correction equation using all of the data, i.e., we pool the eight goods, twelve years, and seventy-six countries. We estimate equation 1 below, where $\Delta \equiv$ the first-difference operator.

$$
\begin{gathered}
\Delta p_{t}^{i m p}=\beta_{1} \Delta s_{t}+\beta_{2} \Delta p_{t}^{\exp }+\sum_{i} \lambda_{i} \Delta s_{t} X_{i}+\gamma e c m_{t-1}+\sum_{i} \alpha_{i} e c m_{t-1} X_{i}+ \\
+ \text { product and country dummies }+\varepsilon_{t} .
\end{gathered}
$$

For the base case estimation, the matrix $X$ is empty. The variables initially included in equation (1) are defined as: 


$$
\begin{aligned}
p_{t}^{i m p} & =\log \text { price of import good in local currency } \\
s_{t} & =\log \text { bilateral exchange rate (importer's currency per unit of exporter's currency) } \\
p_{t}^{\exp } & =\log \text { exporter's price (in the exporting country) } \\
e c m_{t-1} & =\text { error correction term }\left(p_{t-1}^{i m p}-s_{t-1}-p_{t-1}^{\exp }\right),
\end{aligned}
$$

After reporting estimates from this basic equation, we sequentially add variables to the $X$ matrix. This approach allows us to begin with a very simple equation that explains changes in local prices as a function only of changes in the exchange rate and changes in the price of the identical commodity, and an error-correction process. This tells us the contemporaneous or short-term passthrough coefficient and the speed of subsequent adjustment [the negative ECM term]. Next, we look for possible (unconditional) time trends in the degree of short-term passthrough and the speed of adjustment, by interacting a time trend with the exchange rate change and with the ECM term. Finally, we add successive variables (from the list below) that might help explain these two parameters and their trends. We begin with relative income (importer/exporter) because we want to know if passthrough is (unconditionally) stronger for poor countries than rich. Then we proceed to condition on a sequence of further variables, not only to see if they are important determinants in their own right, but also to see if they claim some of the explanatory power of the trend and the income term. Specifically, we sequentially add the following variables to the matrix $X$ in equation (1):

$$
\begin{aligned}
\text { trend } & =\text { trend } \\
\text { income }_{t} & =\log (\text { import country per capita GDP/export country per capita GDP }) \\
\text { tariff }_{t} & =\log (\text { import country tariff levels }) \\
\text { dist } & =\log \text { distance (between importer and exporter }) \\
\text { size }_{t} & =\log (\text { import country GDP/export country GDP }) \\
r \text { waget } & =\log \text { real wage (in import country) } \\
\text { infl }_{t} & =\log \text { inflation (average of previous five year's, in import country) } \\
x r v o l & =\text { standard deviation of previous five year's monthly log changes in bilateral exchange rates } \\
\text { US dummy } & =\text { dummy variable ( } 1 \text { if US is importer, } 0 \text { otherwise). }
\end{aligned}
$$

\section{Results}

For the tables below, we report results for the complete set of commodities. However, in separate appendices, we also report results on a good-by-good basis.

\section{The Determination of Retail Prices of Imported Goods}

Table 1 presents the coefficient estimates for the determination of imported goods prices at the retail stage, as specified in equation 1. We report standard errors corrected for heteroscedasticity and autocorrelation (Newey-West). There are dummy variables for countries as well as commodities. The advantage of allowing dummy variables is that it takes care of any country-specific or commodity-specific omitted variables, such as whether some market are 
more highly competitive than others. The disadvantage, of course, is that it means throwing out a lot of potentially useful variation in the data. It is our hope that our independent variables, such as income, size, and so forth, will explicitly capture much of what would otherwise be heterogeneity in the parameters.

In column 1, the specification includes only the change in the exchange rate, the change in exporter's price, and last period's deviation from the law of one price (and good and country dummies). According to the estimates in column 1, pass-through is highly significant, but far from complete after one year (0.42). The passthrough of changes in the exporter's price is also highly significant, but much smaller in magnitude, a pattern that will hold throughout. The difference between the two kinds of passthrough is large and significant, and so we will not impose the constraint that they are equal as the law of one price would require. ${ }^{18}$

The coefficient on the error correction term captures long-run reversion to absolute price parity. Although highly significant statistically, the estimate of .11 suggests that convergence is quite slow. The half life is 6.1 years $(\ln (.5) / \ln (1-.107))$, somewhat above the 'consensus' noted by Rogoff (1996). In the first year, the failure of the law of one price is apparently due to slow adjustment far more than to a long-run passthrough coefficient that falls short of one. This suggests that sticky prices play a large role, relative to either optimal price discrimination or ruleof-thumb mark-up pricing. We also note that since our data are (a) sampled at a point in time, and (b) disaggregated by product, recent theoretical arguments suggesting that slow convergence may be due to product-aggregation bias, or temporal-aggregation bias, apparently do not apply to these data.

Column 2 reports highly significant downward time trends in both the magnitude of the passthrough coefficient and the magnitude of the ECM term. Remarkably, the trend is estimated to be strong enough to eliminate more than $2 / 3$ of the passthrough coefficient over a ten-year period $\left(10^{*} .053 / .76=.70\right)$. Perhaps the linear trend is not the best functional form, however; after all we don't think that the passthrough coefficient should go negative.

In column 3 we add the income term: the log of relative per capita income (the importer relative to the exporter), interacted both with the change in the exchange rate and the ECM term. At this point the interaction variables in $X$, so far, are: income, and a trend. The result is a highly significant negative effect on the passthrough coefficient, confirming lower passthrough for rich countries than poor. There is no tendency for the time trend in the passthrough coefficient to lose strength when controlling for income; this indicates that one cannot explain the tendency for the coefficient to decline globally simply to a convergence of income levels. (Indeed, Figure 1, a graph of the average income per capita, PPP basis, vis-à-vis the US shows no overall tendency toward income convergence among these countries during the 12 years of the sample.) There is no significant implication of income for the ECM term, the speed of adjustment.

In column 4 we control for tariffs and distance. Both coefficients have the hypothesized negative signs, but neither is statistically significant. Distance does, however, have a highly significant effect on the ECM term, suggesting quite sensibly that transport costs slow down the

\footnotetext{
${ }^{18}$ It is possible that there is an element of endogeneity to the export prices - that a depreciation of the Moroccan currency against the French franc shows up partly as a decline in the price of the export product in France, not just as an increase in the price in Morocco. But as almost all the exporters are large countries, we guess that this effect may be small.
} 
speed of adjustment, as in a sticky price model, or possibly a rule-of-thumb markup pricing model.

Size, introduced in column 5, is not statistically significant. Even if it had been, Figure 2 shows no sign of convergence in the size of countries' economies within our sample. Thus growth by small countries does not appear to have been the source of declining passthrough in our sample. The coefficient on wages in column 6 again is of the hypothesized sign (for the distribution and retail models) but not statistically significant.

Long-term inflation, in columns 7-8, is significant, especially if we do not control for exchange rate variability at the same time. The trend term falls somewhat when controlling for inflation. Furthermore, long-term inflation is also a significant determinant of the ECM term, signifying that adjustment takes place more quickly in an inflationary environment. And the trend in the ECM term also falls sharply and loses significance when controlling for inflation. Thus we conclude that the inflationary environment is an important determinant of the speed and degree of passthrough, and that the decline in inflation during the 1990s is one reason for the decline in both of these parameters.

To complete the attribution of declining pass-through to a less-inflationary environment, as in Taylor (2000), we should document the extent to which inflation did indeed decline in our sample. Figure 3 depicts what has happened to average inflation for the 76 countries considered here during the twelve years of this study. In accord with conventional wisdom, inflation has been falling everywhere. Both the mean and its cross-country standard deviation have declined since 1990. ${ }^{19}$ In our sample of countries, average (un-weighted) inflation fell from 22 percent per year in 1990 to 6 percent in 2001. According to the estimates in the table, this magnitude of decline in average inflation (18 percentage points) implies a decline in the average pass-through coefficient of about six percentage points $\left(.06=18^{*} .36\right)$. The decline for the median country is smaller, from .07 in the first part of the sample to .03 in 2000 and 2001 . Nevertheless, the decline in inflation is apparently one component of the overall observed decline in pass-through. Figure 4 illustrates the contrast between the passthrough coefficient in high-inflation countries and low inflation countries, and between the first half of the sample period and the lowerinflation second half.

Poor countries have historically had higher tariffs and higher inflation rates than rich countries. One might have therefore expected that the estimated effect of the income term would change when controlling for such factors -- that it would have been biased upward before controlling for tariffs, and downward before controlling for inflation. But that does not happen in Table 1.

Exchange rate variability is also a significant determinant of the ECM term, but with a sign that indicates a positive effect on the speed of adjustment, the opposite from the KrugmanFroot-Klemperer-Taylor prediction. It may be that this term is capturing changing long run trends in the same way that the inflation term is.

\footnotetext{
${ }^{19}$ Indeed, the decline in the average inflation rate in industrial countries has been steady over three decades: from $12 \%$ in the second half of the 1970 s to $2 \%$ in the second half of the 1990 s. The average inflation rate among developing countries has moved less monotonically, but also has declined substantially more recently (from $25 \%$ in the second half of the 1970 s to $13 \%$ in the second half of the 1990s). Tytell and Wei (2003).
} 
Finally, the dummy representing when the US is an importer is significant. This confirms the consensus that pricing to market is more common in the world's largest national market. Exporters to the United States absorb exchange rate fluctuations in profit margins, rather than passing them through to their customers. Because our data are so narrowly defined, it is not possible that this finding is due to the sort of composition differences to which others have attributed findings of low passthrough to US price indices. ${ }^{20}$ [It should be noted, however, that we are talking about only three products; the other goods are US products and so are excluded.]

For robustness, Appendix Table 1 drops some observations where one might have qualms about the data, such as prices of alcoholic beverages in Moslem countries. Most of the results are qualitatively the same as before. Appendix Table 5 reports the same regressions, but without the country dummies. Again, most of the results are qualitatively similar.

The next order of business is to examine other points along the chain of pricing passthrough. The complete chain runs from the country of export to dockside in the country of import, to retail in the country of import, to locally produced competing goods, to the general price level. So far we have looked only at the second passthrough, to the import price at the retail level.

\section{Determination of prices of local substitutes}

Tables 2, and Appendix tables 2 and 6, show the next stage, passthrough to the prices of locally produced goods that are competitive with the specific imported goods in our sample. For example, we use a local cigarette brand as the relevant substitute for Marlboros, local beer as a substitute for imported alcoholic beverages, a local newspaper as a substitute for Time magazine, and so on. (Appendix Table A gives the complete list of commodities and substitutes.)

Obviously these goods are not perfect substitutes for the imports. As one would expect, the $\mathrm{R}^{2}$ is somewhat smaller; and the passthrough of exchange rate changes to the local substitutes is less than the passthrough to imports. But it is still highly significant, and only slightly smaller [.37 as compared to .40 , in the complete sample with country dummy variables; or .56 as compared to .58 , in the version that includes country dummies]. ${ }^{21}$ The main difference is in the ECM parameter: adjustment is far slower for the local substitutes than for the imports themselves. Furthermore the downward trend in the passthrough coefficient is even stronger than for imports themselves. (The trend in the ECM coefficient suggests that adjustment has been speeding up in the case of the local substitutes, when estimated with the full sample and dummies.)

As before, the relative income term is statistically significant, suggesting that richer countries have lower passthrough coefficients; but the effect is only half as strong as for the case of import prices. Distance appears significant, but paradoxically appears to have the effect of increasing the passthrough coefficient. Size has a consistent and significant effect on the ECM term: larger countries exhibit slower adjustment, as expected. Real wages have the negative effect on the passthrough coefficient that is hypothesized -- higher labor costs create more of a margin insulating prices of local substitutes from import competition -- and are often highly significant statistically. Long-term inflation has the hypothesized positive effect on the degree of

\footnotetext{
${ }^{20}$ Knetter (1993) and Campa and Goldberg (2002). [Otani, Shiratsuka and Shirota (2003) similarly conclude that composition cannot be the entire explanation for the decline of passthrough, for the case of the Japanese market.]

${ }^{21}$ That the passthrough coefficient is similar suggests higher substitution than one might expect.
} 
contemporaneous passthrough, and is highly significant. Exchange rate variability again has the unexpected effect of increasing the speed of subsequent adjustment. As was also the case with import prices, some but not all of the downward trend in passthrough to local prices is explained, by long-term inflation.

Some of the differences relative to import prices are noteworthy: that distance, size, and wages are now important determinants of the level as short-run passthrough, whereas previously distance and wages mattered only for the speed of adjustment.

Without country dummies (Appendix Table 6), the unconditional passthrough coefficient is higher (.56), with an equally strong downward trend. Most of the results are as before. Richer countries clearly have a lower passthrough coefficient [but appear to have a faster speed]. Tariffs, wages, size and long-term inflation have strong effects on the level of passthrough. Size works strongly to slow down the speed of adjustment. Exchange rate variability again has the unexpected significant effect of increasing the speed. [Distance has a puzzling positive coefficient. The US dummy appears very significant and of the unexpected sign; but this should probably be discounted because it is estimated from only three non-U.S. export goods -cointreau, cognac and vermouth -- all three of which unfortunately have the same domestic competitor good, beer.]

\section{Determination of the CPI}

Next we leap to the highest level of aggregation: the determination of the consumer price index, in Table 3 and Appendix tables 3 and 7. As one would expect, there is a clear fall in the magnitude and significance of passthrough - but the coefficient remains fairly strong (.28-.59) and highly significant. The estimated speed of adjustment falls enough to lose statistical significance. The downward trend in the passthrough coefficient is just as strong as before. But now there is also a significant trend toward a slower speed of adjustment. The speed of adjustment is slower the higher is the country's income, when not conditioning on other variables, as expected. But there is no indication that the passthrough coefficient depends (unconditionally) on income. And when conditioning on wages and the monetary variables, passthrough is actually significantly higher in rich countries. [The speed of adjustment also increases conditionally with income, in Appendix table 7 which excludes the country dummies.]

Tariffs and distance are both estimated to have negative effects on the passthrough coefficient, as hypothesized. Size is significant, but of the wrong sign: big countries appear to experience more passthrough. The effect of wages is highly significant and of the right sign: labor costs reduce passthrough to the CPI. Both monetary variables are highly significant and of the right sign: long-term inflation raises the level and speed of passthrough to the CPI, while long-term exchange rate variability lowers the level and speed of passthrough.

The results are similar when the country dummies are omitted (Appendix Table 7). The most notable difference is that higher wages are now seen to slow down the speed of adjustment significantly.

\section{Determination of Import Prices at the Dock}

Finally, we go back to the determination of the prices of our narrowly defined import goods when they first arrive in the country. These data are disaggregated unit value prices. The prices are observed, figuratively speaking, "at the dock;" in other words, the stage before the 
retail prices that we began by examining above. Figure 5 shows that the passthrough coefficient is higher for the prices at the dock than for the same imports at retail, higher for retail import prices than for local competitor prices, and higher for local competitor prices than for the aggregate price index. This is precisely what we would expect, but it is nice to see it.

Table 4, and Appendix Tables 4 and 8, seek to explain passthrough to unit value import prices, with the same variables that we used to explain other local price measures. [To the extent that price discrimination is the right model of price determination, we might expect that these incountry wholesale prices would exhibit similar pricing to market behavior as do the retail prices of the same goods. But to the extent that retailers follow inertial sticky-price rules, we might expect that passthrough will be more immediate at the wholesale level.]

The table shows that passthrough to prices in the port behaves quite differently than to

retail. It is not surprising to find a higher overall passthrough coefficient, as noted: .53-.68. But, more strikingly, there is an upward trend in the coefficient, at .04 per year, and also in the speed of adjustment. The estimated trend in the passthrough coefficient, in theory, should be strong enough to reach 1.0 -- complete passthrough -- by the end of the sample period. (Extrapolating the estimated trend in the ECM term, by contrast, suggests it would take more than a century to reach instantaneous adjustment.) Moreover, income is highly significant, appearing to suggest that richer countries have higher passthrough. Perhaps wholesale markets are more competitive and less regulated in rich countries than poor.

More in line with our a priori reasoning, tariffs, distance and size all work to reduce passthrough significantly. [Distance paradoxically works to increase the speed of adjustment, however.]. Unlike the retail case, the effect of inflation on the passthrough coefficient is of the wrong sign, and insignificant. Evidently the rising importance of pricing to market, and the role of a less inflationary environment, are entirely retail phenomenon.

Unit value data are traditionally viewed as less reliable than other price data. That is one reason why we have placed these results last. But this suspicion is less justified for prices of highly disaggregated goods such as we are using, than it would be for aggregate indices of import prices. Furthermore, that we have a strong passthrough estimate and that we are able to identify specific significant determinants of it, suggest that the unit value prices may not be subject to large measurement error.

\section{Passthrough in Developing Countries}

Our last task in this paper is to break out the results for low-income countries, separately from high-income countries. Our motivations for doing so were laid out at the beginning, including the lack of detailed econometric scrutiny that they have received in the past. Moreover, the results in the preceding section have confirmed the traditional wisdom that the passthrough coefficient varies with per capita income. Perhaps the most striking lesson of this paper emerges only in this section: some of the aspects of the determination of local retail prices that we have identified in the preceding sections turn out to be phenomena that apply primarily or even exclusively to developing countries.

In Tables 5-8 we report for each coefficient the base-case estimate from the sample of high-income countries, and then the estimate for developing countries (defined as either lowincome or middle-income) expressed as a deviation from the base case. Table 5 reports the determination of retail import prices. Equation 1 shows that for developing countries, the level 
of the (unconditional) passthrough coefficient is almost four times as high as it is for highincome countries. In Table 6, for competitors' prices, and Table 7, for the CPI, the passthrough coefficient in poor countries is an even greater multiple of that in rich countries, on the order of ten times as high. Table 8 , for unit values, is the exception. The estimate for the passthrough coefficient shows no significant difference between rich and developing countries.

In Equation 2 of Table 5, the clear downward trend in the passthrough coefficient during the sample period is twice as high for developing countries as for rich countries. But because the initial level of passthrough is high, the trend is not enough to eliminate the poor-rich differential by the end of the period. The downward trend is also substantially stronger for poor countries in the determination of competitors' prices and the CPI. Indeed, in the case of the CPI, the downward trend in the pass-through coefficient for developing countries is strong enough to eliminate all of the difference by the end of the 12-year sample period.

The ECM term in these tables suggests significantly faster adjustment for poor countries than rich in the case of retail imported goods (Equation 1 of Table 5). [The difference is not significant in the case of local competitors' prices or unit values, and is significantly slower in the case of the CPI.] While the ECM term has no trend at all for rich countries, there is a strong downward trend in the speed of adjustment for developing countries, which by the end of the period is again sufficient to outweigh the initial difference in speeds.

Equation 3 of Table 5 suggests that, even within the set of developing countries alone, higher income continues to mean lower passthrough to import prices. The effect whereby higher wages reduce the passthrough coefficient, however, turns out to be only a property of rich countries. The effect whereby greater distance means significantly slower adjustment turns out apply equally to both sets of countries. The breakdown sheds light on the earlier finding that long-term exchange rate variability increases the speed of adjustment: this turns out to be a property only of developing countries. For rich countries, exchange rate variability delays adjustment, as hypothesized by Froot-Klemperer-Krugman-Taylor. Most likely the result for poor countries is dominated by time variation in trends, even though we are also conditioning on long-term inflation variability, and even though we have removed a (constant) trend from both monetary measures before computing variability.

In the determination of local competitors' prices, Table 6 , the difference in trends is enough to eliminate more than half of the difference in passthrough by the end of the sample period. The phenomenon whereby long-term high inflation increases passthrough turns out to be a property of rich countries. Controlling for the effect of lower inflation all but eliminates the downward trend in rich-country passthrough to competitors' prices. But we do not explain the downward trend for developing countries, either with inflation or with any other variables.

[Long-term inflation appears to slow down adjustment of competitor prices for rich countries, but only for them. (This puzzle applies also to the CPI in the next table.) Controlling for the change in inflation in turn all but eliminates the apparent trend of acceleration of the ECM parameter. Again, the effect whereby exchange rate variability appears to raise the speed of adjustment turns out to be a phenomenon of the developing countries alone.]

In the determination of the CPI, Table 7, as already noted, the downward trend in passthrough for developing countries is strong enough to eliminate the entire differential vis-àvis rich countries. In this case we can identify the main reason for the downward trend: longterm exchange rate variability has a strong negative effect on passthrough for developing 
countries (the opposite of the effect for rich). When controlling for exchange rate variability, the downward trend for poor countries all but disappears. This suggests that the trend estimates are capturing low passthrough during the currency crises of the latter half of the sample period. Tariffs and distance both turn out to reduce the passthrough coefficient even more strongly for developing countries than they do for rich countries. But the pattern whereby tariffs and distance slow down the speed of adjustment turns out to apply only to rich countries.

Generalizing across the three different price measures, the passthrough coefficient has traditionally been higher for lower-income countries, but a strong downward trend in the coefficient during the course of the 1990s eliminated much of the gap. A similar story can be told for the speed of adjustment in the case of import prices. But in the case of the CPI, it is rich countries that have the faster adjustment. Tariffs and distance have highly significant downward effects on passthrough to the CPI. The monetary variables have the hypothesized effect for developing country CPIs, but not necessarily in other cases.

Those results pertain to the various retail prices. The case of passthrough to the prices in the port, reported in Table 8 is a different story. Here there is not much difference in the passthrough coefficient between rich and poor countries (although the speed of adjustment is higher for poor countries than rich). Both have passthrough coefficients in the neighborhood of .7. Tariffs have the hypothesized significant negative effect on passthrough for rich countries (when controlling for other variables like size), but only for rich countries. Rich countries are also the only ones to show the unexpected negative effect of long-term inflation on passthrough to unit prices and positive effect of exchange rate variability on the speed of adjustment.

\section{Other Extensions} drafts.

We have pursued a number of extensions in response to comments from readers of earlier

We tried adding on the right-hand side of the import price equation measures of domestic inflation, particularly changes in prices of competing goods and changes in the cost of domestic inputs (wages). We had omitted them from our basic results, because we had already been using the former as the dependent variable in the local prices regression and the latter as an interactive component of the Balassa-Samuelson variable. But this is not a good enough reason, in that theory requires these variables. The least we can do to respond to concerns that they are missing from the equation -- and, worse yet, that such measures of domestic inflation are correlated with exchange rate changes -- is to try adding them to the right-hand side. We did try this, and found that the basic conclusions about the coefficient trend did not change. ${ }^{22}$

Three other important extensions are to look for:

(1) asymmetric effects between appreciation and depreciation

(2) threshhold effects whereby the proportionate effect on prices of large devaluations is proportionately different -- presumably, smaller -- than for small devaluations.

(3) cyclical factors, as an additional determinant on the list.

\footnotetext{
${ }^{22}$ Charles Engel suggested this line of exploration to us.
} 
The salient motivation in all three cases is the emerging market currency crashes of the late 1990s. The observation that inflation rates did not rise nearly to the extent of currency depreciation during the recent crises in Asia and Latin America does not necessarily imply a consensus that the pass-through coefficient has declined structurally. Goldfajn and Werlang (2000) argue that the pass-through measured during these crises may be lower than during a normal, tranquil time. The reason is that recession could act to depress domestic prices, hence generating a spurious appearance that domestic prices do not respond much to exchange rate depreciation. Carranza, Galdon-Sanchez, and Gomez Biscarri (2004) find evidence of such asymmetries in passthrough of devaluation to the CPI in 15 emerging market countries.

Thus we checked with our data if one still saw a secular decline in the degree of passthrough among developing countries after controlling for the effect of crisis-related recession episodes, via asymmetry and/or a business cycle effect. For comparison with previous tables, we report results for each stage (retail, local competitor, CPI, and unit value) in Appendix Tables 912. In Appendix Tables 13-16, we present the results for the rich-poor comparison.

The output gap interacted with exchange rate change appears statistically significant, but the direction is opposite the hypothesized effect (passthrough appears higher in recessions, not lower). The threshold effect, while significant, goes the wrong way: increases in the exchange rate above $25 \%$ are found to have proportionately larger passthrough effects, not smaller. We did find strong evidence of asymmetry. In fact we cannot reject the hypothesis that appreciation is not passed through at all, suggesting downward price rigidity. This is an interesting finding. But the significant downward trend in the passthrough coefficient remains nonetheless. In short, the decline in passthrough during this period does not appear to be merely an artifact of the highprofile currency crashes in emerging markets.

\section{To Conclude}

We have produced a lot of results, but we still have things to do.

\section{Next steps}

A number of possible extensions remain for future work. It would be nice to ground the estimation in a theoretical model of the passthrough coefficients for prices of imports and local substitutes, simultaneously. The parameters should be modeled as depending, at a minimum, on a parameter representing the magnitude of barriers to arbitrage and another representing the competitiveness of local markets.

We could relax the constraint that passthrough is complete in the long run. The spirit of the models of optimal price discrimination such as Dornbusch (1987), Gagnon and Knetter (1995), and others is that passthrough is incomplete even in the very long run and even for the most disaggregated of products. One possible empirical extension is to apply the TAR (threshold autoregressive) technique, to reflect that arbitrage should in theory work within a band determined by barriers (tariffs, transport, distribution costs, etc.), rather than as a linear autoregressive process.

We could allow for correlation of errors across commodities. In Appendices a1-a8, b1$\mathrm{b} 8$, and $\mathrm{c} 1-\mathrm{c} 8$, we report results we have obtained for individual commodities. They show a lot of variation (which we attribute to garden-variety estimation error). The results reported here were for the eight commodities pooled together, though with commodity-specific dummies. 


\section{Summary of conclusions}

To summarize our findings,

1. As one would expect, passthrough of exchange rate changes is greatest in the determination of prices of imported goods at the dock, is less to prices of the same goods at the retail level, somewhat less to prices of local substitutes for such goods, and still less to the CPI.

2. Nevertheless, even for import prices at the dock (and even in developing countries), passthrough is not complete and instantaneous. We would reject the idea that the Burstein, Eichenbaum, and Rebelo hypothesis constitutes the entire explanation for incomplete passthrough to the CPI, though nothing rules out that it could explain part.

3. Transport costs (as proxied by distance) are an important barrier to arbitrage reducing or slowing passthrough at all four stages -- dockside imports, retail, competitors' prices, and the CPI.

4. Tariffs are another important barrier.

5. There is clear evidence of stickiness: that is, inertia, followed by slow adjustment, as reflected in an error-correction process;

6. Importers do indeed "price to market" in that passthrough to retail import prices is not just incomplete, but is broadly similar to passthrough to the prices of local substitutes. (There is no need to choose between the price discrimination model and the price stickiness model; both are probably important.)

7. There is a little evidence of a size effect -- passthrough is higher or faster in a small country than in a large one - but much less than one would expect.

8. A particular example, for retail prices, is that passthrough is much smaller into the United States import market than into other countries. This result cannot be attributed to composition effects, because the goods are so disaggregated.

9. Per capita income is perhaps the second most robust determinant of the passthrough coefficient (after distance). It generally does not lose value when conditioning on wages (but it sometimes loses some explanatory power when conditioning on long-term inflation). As implied by the "small open economy model," poor countries have traditionally experienced higher passthrough.

10. The monetary climate is also important: passthrough coefficients are significantly higher in an environment of high inflation. Often they are also influenced by an environment of transitory exchange rate fluctuations.

11. There is some evidence that passthrough to price of imports on the dock has actually gone up (perhaps due to declining transportation costs).

12. Otherwise, passthrough to retail prices (or imports, substitutes, and the CPI) did indeed experience a substantial downward trend during the 1990s - both a decrease in the contemporaneous coefficient and a (small) decrease in the speed of subsequent adjustment.

13. In particular, retail passthrough coefficients have historically been much higher in poor countries than in rich ones, but the coefficient in poor countries declined significantly in the 1990s. The downward trend among rich countries is much less, and for the CPI is not statistically significant.

14. Some of the downward trends in the degree of passthrough and speed of adjustment can be explained by changes in its determinants, but some of the trend remains unexplained.

15. One factor in explaining part of the decline in the passthrough coefficient in the 1990s is a decline in the inflationary environment. 
16. The hypothesized monetary variables are particularly relevant in explaining the decline in the passthrough to developing-country CPIs.

17. Higher wages have a strong negative effect on passthrough to the local competitors' prices and the CPI, supporting the hypothesized importance of distribution and retail costs.

Controlling for wages reverses the tendency for passthrough to the CPI to decline as income grows (compare equations 5 and 6 in Table 3). A possible interpretation is that the role of distribution costs in pricing to market may become increasingly important as countries achieve higher incomes, due to the Balassa-Samuelson-Baumol effect. 


\section{$\underline{\text { References }}$}

Aw, Bee-Yan, 1993, "Price Discrimination and Markups in Export Markets," Journal of Development Economics 42, 315-336.

Bacchetta, Philippe, and Eric van Wincoop, 2002, "Why Do Consumer Prices React Less than Import Prices to Exchange Rates?” NBER Working Paper No. 9352, Nov. Journal of the European Economic Association 2003.

Bank for International Settlements, 2002, $72^{\text {nd }}$ Annual Report, Basle.

Borensztein, Eduardo, and Jose De Gregorio, 1999, "Devaluation and Inflation after Currency Crises," International Monetary Fund.

Burstein, Ariel, Martin Eichenbaum, and Sergio Rebelo, 2002, "What Are Rates of Inflation So Low After Large Devaluations?" NBER, January.

Burstein, Ariel, Martin Eichenbaum, and Sergio Rebelo, 2003. "Large Devaluations and the Real Exchange Rate," IMF seminar, September.

Burstein, Ariel, Joao Neves and Sergio Rebelo, 2003 "Distribution Costs and Real Exchange Rate Dynamics During Exchange-Rate-Based-Stabilizations,” Journal of Monetary Economics, 50: 1189-1214.

Campa, Jose, and Linda Goldberg, 2002, "Exchange Rate Pass-Through into Import Prices, A Macro or Micro Phenomenon?" NBER WP no. 8934.

Campa, Jose, and Linda Goldberg, 2004, "Do Distribution Margins Solve the Exchange Rate Disconnect Puzzle?" forthcoming, Federal Reserve Bank of New York.

Carranza, Luis, Jose Enrique Galdon-Sanchez, and Javier Gomez Biscarri, 2004, "Exchange Rate and Inflation Dynamics in Dollarized Economies,” FCEE WP no. 10/04,, Universidad de Navarra, Spain, May.

Chen, Shiu-Sheng, and Charles Engel, 2004,’Does ‘Aggregation Bias' Explain the PPP Puzzle,” NBER Working Paper No. 10304.

Choudhri, Ehsan, and Dalia Hakura, 2001, "Exchange Rate Pass-Through to Domestic Prices: Does the Inflationary Environment Matter?” IMF Working Paper WP/01/194.

Corsetti, Giancarlo, and Luca Dedola, 2002, “Macroeconomics of International Price Discrimination,” June.

Devereux, Michael, and Charles Engel, 2002, "Exchange Rate Pass-Through, Exchange Rate Volatility and Exchange Rate Disconnect," Carnegie-Rochester Conference (Nov. 2001); March.

Devereux, Michael, Charles Engel, and Peter Storgaard, 2002, "Endogenous Exchange Rate Pass-Through When Nominal Prices Are Set in Advance," CEPR Discussion Paper 3608, October.

Devereux, Michael, and James Yetman, 2002, "Price Setting and Exchange Rate Pass-Through: Theory and Evidence," Hong Kong Institute for Monetary Research Working Paper No. 22/2002, December.

Dornbusch, Rudiger, 1987, "Exchange Rates and Prices," American Economic Review, 77, 1, March , 93-106.

Engel, Charles, “Accounting for US Real Exchange Rate Changes,” Journal of Political Economy, vol. 107, 507-38.

Feenstra, Robert, 1989, "Symmetric Pass-Through of Tariffs and Exchange Rates Under Imperfect Competition: An Empirical Test," Journal of International Economics, 27, no. 1,2, August, pp 25-45. 
Frankel, Jeffrey, 1984, "The Theory of Trade in Middle Products: Extension," American Economic Review, June.

Froot, Kenneth, and Paul Klemperer, 1989, "Exchange Rate Pass-Through When Market Share Matters," American Economic Review 79, no. 4, Sept., 637-54.

Gagnon, Joseph, and Michael Knetter, 1995, "Markup Adjustment and Exchange Rate Fluctuations: Evidence from Panel Data on Automobile Exports," Journal of International Money and Finance, 14, no. 2 , April, 289-310.

Gagnon, Joseph, and Jane Ihrig, 2004, "Monetary Policy and Exchange Rate Pass-Through,” International Journal of Finance and Economics, vol. 9 no. 4, October, 315-338.

Ghosh, Atish R. and Holger Wolf, 1995, "Pricing in International Markets: Lessons from the Economist," March.

Goldberg, Pinelopi, and Michael Knetter, 1997, "Goods Prices and Exchange Rates: What Have We Learned?" Journal of Economic Literature XXXV, September, 1243-1272.

Goldfajn, Ilan, and Sergio Werlang, 2000,"The Pass-Through from Depreciation to Inflation: A Panel Study," Economics Department, PUC-Rio, Texto Para Discussao No. 424.

Ho, Corrinne, and Robert McCauley, 2003, "Living with Flexible Exchange Rates: Issues and Recent Experience in Inflation Targeting Emerging Market Economies," Bank for International Settlements Working Paper 130, Basel.

Imbs, Jean, Haroon Mumtaz, Morton O. Ravn, Helene Rey, 2002, "PPP Strikes Back: Aggregation and the Real Exchange Rate,“ NBER Working Paper No. 9372, December.

Kasa, Kennth, 1992, “Adjustment Costs and Pricing-to-Market: Theory and Evidence,” Journal of International Economics, 32, no. 1-2, Feb., pp. 1-30.

Knetter, Michael, 1989, "Price Discrimination by U.S. and German Exporters," American Economic Review, 79, no. 1, March 198-210.

Knetter, Michael, 1993, “International Comparisons of Price-to-Market Behavior," American Economic Review 83, no. 3, June, 473-86.

Kreinen, Mordechai, 1977, "The Effect of Exchange Rate Changes on the Prices and Volume of Foreign Trade," International Monetary Fund Staff Papers, July, 24, no. 2, 297-329.

Krugman, Paul, 1987, "Pricing to Market When the Exchange Rate Changes," in Sven Arndt and J. David Richardson, eds., Real-Financial Linkages Among Open Economies, (MIT Press: Cambridge), 49-70.

Krugman, Paul, 1989, "The Delinking of Exchange Rates from Reality," Chapter 2 in Exchange Rate Instability, MIT Press.

Magee, Stephen, 1973, "Currency Contracts, Passthrough and Devaluation,” Brookings Papers on Economic Activity, 1, 202-23.

Maloney, William, 1994, "Exchange Rate Uncertainty and the Law of One Price," CIBER [CIDER?] working paper 94-010, October

Mann, Catherine, 1986, “Prices, Profit Margins, and Exchange Rates,” Federal Reserve Bulletin, 72, June, 366-79.

Marston, Richard, 1990, "Pricing to Market in Japanese Manufacturing," J. of International Economics 29, Dec., 217-36.

Ohno, Kenichi, 1989, “Export Pricing Behavior of Manufacturing: A US-Japan Comparison,” International Monetary

Fund Staff Papers 36, Sept, 550-79. 
Otani, Akira, Shigenori Shiratsuka, and Toyoichiro Shirota, 2003, "The Decline in the Exchange Rate PassThrough: Evidence from Japanese Import Prices," Monetary and Economic Studies, Bank of Japan, vol. 21, no. 3, October; p.53-81.

Parsley, David, 1993, “Exchange Rate Pass-Through: Evidence from Aggregate Japanese Exports,” Southern Economic Journal, 60, no. 2, October, 454-62.

Parsley, David, 2002, “Pricing in International Markets: A ‘Small Country’ Benchmark,” Vanderbilt University.

Parsley, David, and Shang-Jin Wei, "A Prism into the PPP Puzzles: The Micro-Foundations of Big Mac Real Exchange Rates,” NBER Working Paper No. 10074, Nov. 2003.

Rogoff, Kenneth, 1996, “The Purchasing Power Parity Puzzle, J. Economic Literature, 34: (2), 647-68.

Saiki, Ayako, 2004, "The Change in Inflation Persistence and Exchange Rate Pass-Through for Inflation Targeting Countries," Essay 2 in Ph.D. dissertation, Brandeis University

Taylor, John, 2000, "Low Inflation, Pass-Through and the Pricing Power of Firms," European Economic Review, 44, no. 7, June, 1389-1408.

Tytell, Irina, and Shang-Jin Wei, 2003, "Does Financial Globalization Induce Better Macroeconomic Policies?" forthcoming, NBER working papers.

Yang, Jiawen, 1997, "Exchange Rate Pass-Through in US Manufacturing Industries," Review of Economics and Statistiges, 95-104. 
Figure 1: Average log per capita income relative to the US

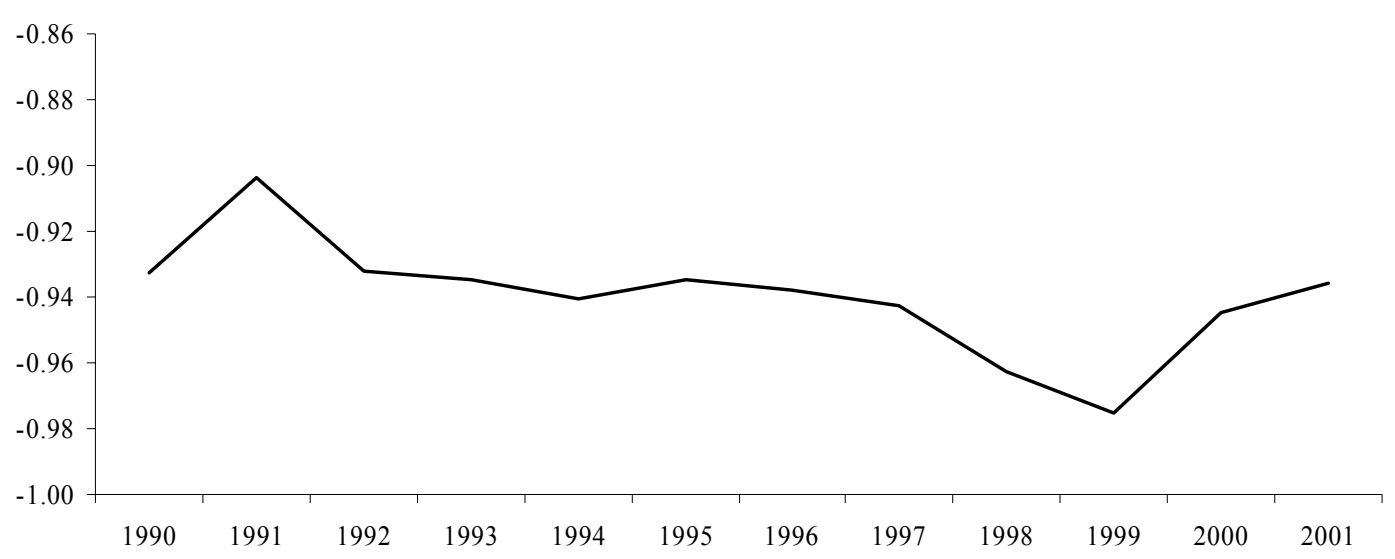

Figure 2: Average log Real GDP relative to the US

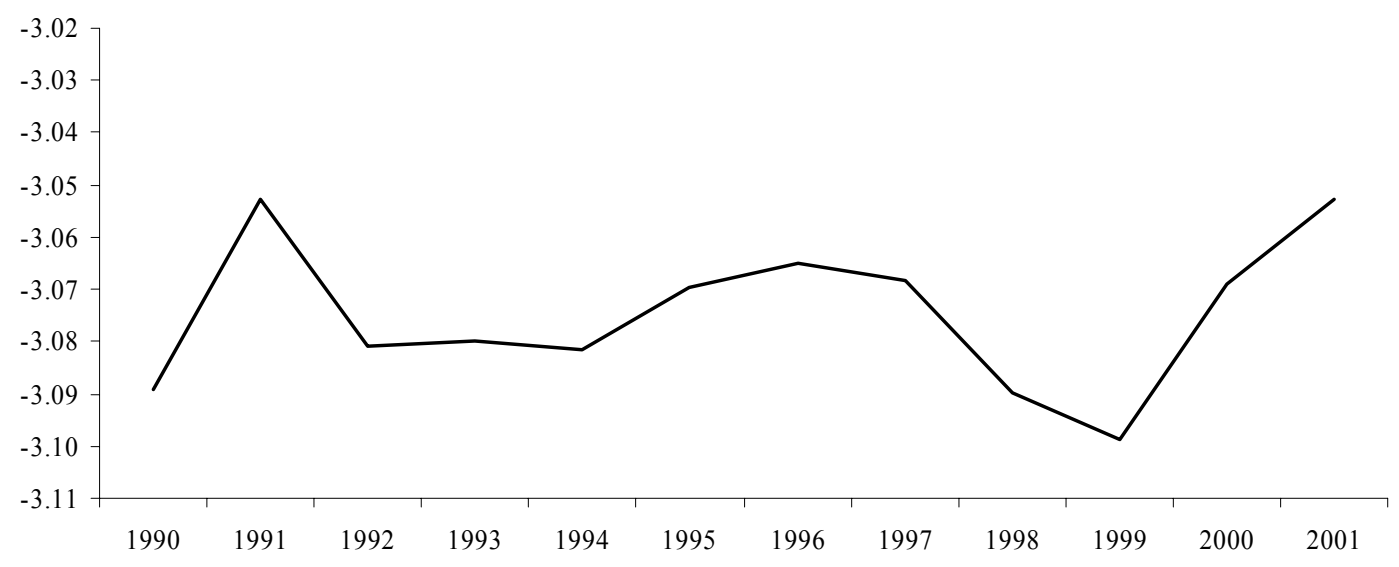

Figure 3: Inflation Decline in Sample

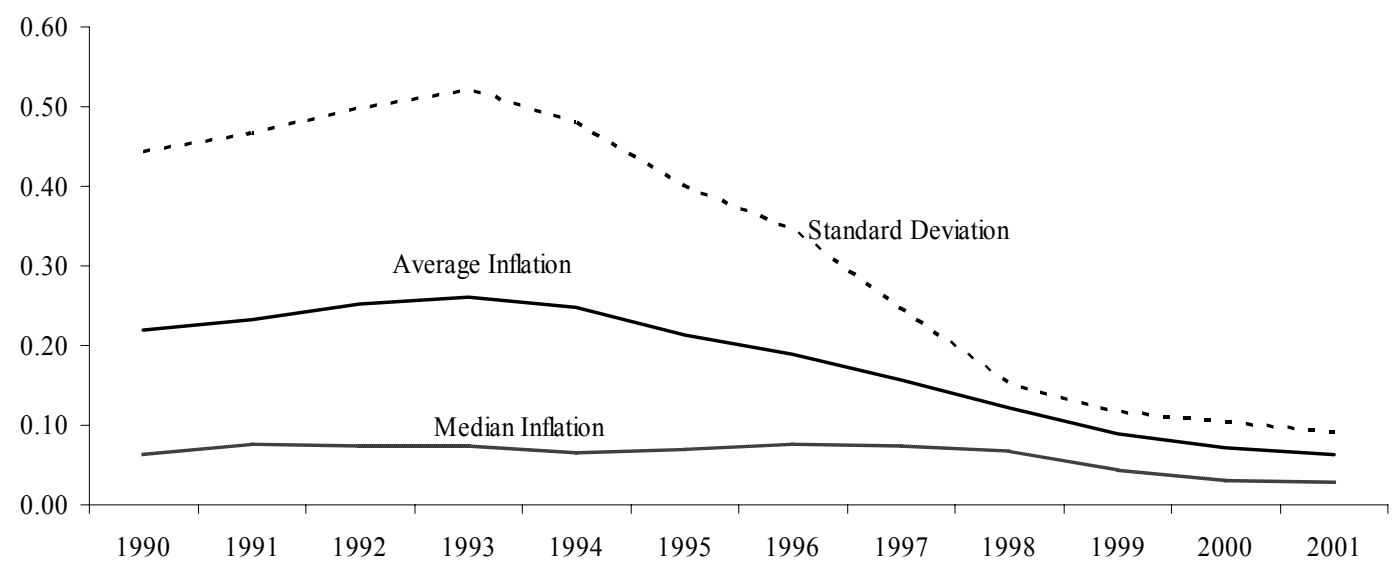


Figure 4: Passthrough coefficient and Inflation

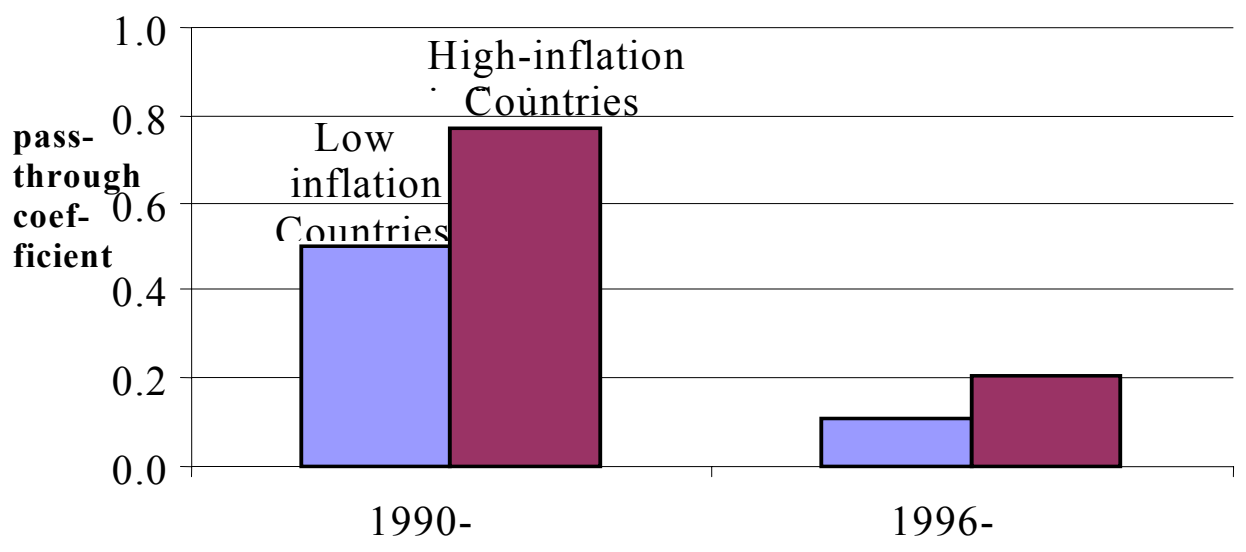

Figure 5: Exchange Rate Passthrough

to Domestic Prices

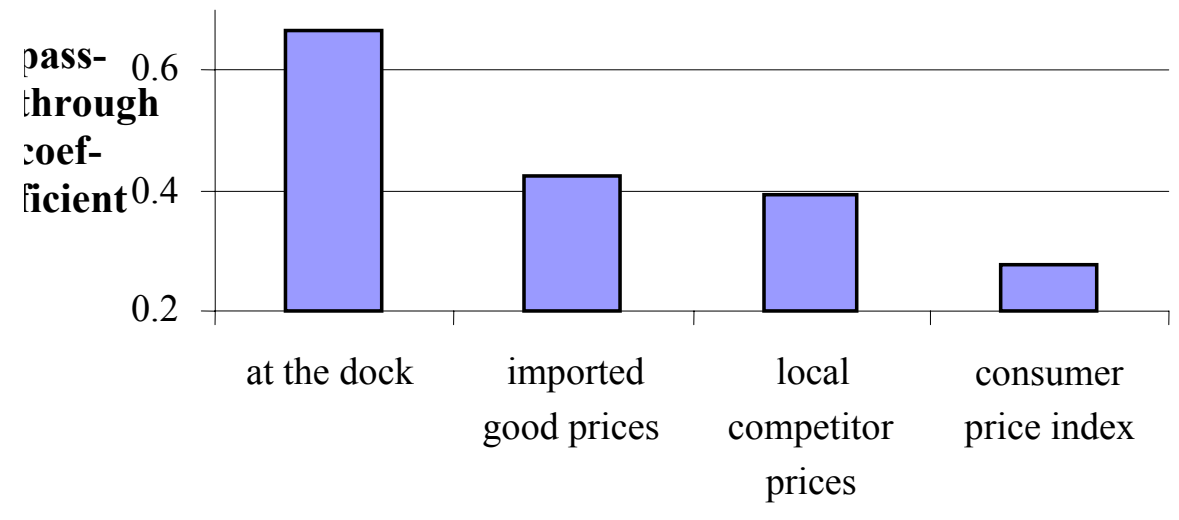


Table A: Goods Included

\begin{tabular}{llll}
\hline Goods (from Supermarket) & Exporting Country & Competing Prices from EIU \\
\hline 1. & Marlboro Cigarettes (pack of 20) & United States & Local brand cigarettes \\
2. & Coca-cola $(1$ liter) & United States & Mineral water \\
3. & Cognac, French VSOP $(700 \mathrm{ml})$ & France & Local brand beer \\
4. & Gilbey's Gin, or equivalent $(700 \mathrm{ml})$ & United States & Local brand beer \\
5. & Time magazine & United States & Daily local newspaper \\
6. & Kodak Color Film $(36$ exposures $)$ & United States & Compact disk album \\
7. & Cointreau Liqueur $(700 \mathrm{ml})$ & France & Local brand beer \\
8. & Martini \& Rossi Vermouth $(1$ liter $)$ & Italy & Local brand beer \\
\hline
\end{tabular}

Table B: Countries Included

\begin{tabular}{llllll}
\hline 1 & Argentina & 20 Egypt & 39 Libya & 58 Saudi Arabia \\
2 & Australia & 21 Finland & 40 Luxembourg & 59 Senegal \\
3 & Austria & 22 France & 41 Malaysia & 60 Singapore \\
4 & Azerbaijan & 23 Gabon & 42 Mexico & 61 South Africa \\
5 & Bahrain & 24 Germany & 43 Morocco & 62 South Korea \\
6 & Bangladesh & 25 Greece & 44 Netherlands & 63 Spain \\
7 & Belgium & 26 Guatemala & 45 New Zealand & 64 Sri Lanka \\
8 & Brazil & 27 Hong Kong & 46 Nigeria & 65 Sweden \\
9 & Cameroon & 28 Hungary & 47 Norway & 66 Switzerland \\
10 & Canada & 29 Iceland & 48 Pakistan & 67 Taiwan \\
11 & Chile & 30 India & 49 Panama & 68 Thailand \\
12 & China & 31 Indonesia & 50 Papua N. Guinea & 69 Tunisia \\
13 & Colombia & 32 Iran & 51 Paraguay & 70 Turkey \\
14 & Costa Rica & 33 Ireland & 52 Peru & 71 U. Arab Emirates \\
15 & Cote d'Ivoire & 34 Israel & 53 Philippines & 72 U. Kingdom \\
16 & Croatia & 35 Italy & 54 Poland & 73 U. States \\
17 Czech Republic & 36 Japan & 55 Portugal & 74 Uruguay \\
18 & Denmark & 37 Jordan & 56 Romania & 75 Venezuela \\
19 & Ecuador & 38 Kenya & 57 Russia & 76 Vietnam \\
\hline
\end{tabular}

Table C: Unit Value Series

\begin{tabular}{|c|c|c|}
\hline Goods & \multicolumn{2}{|c|}{ SITC Code } \\
\hline 1. Cigarettes & Cigarettes (tobacco) (4 digit) under Tobacco, manufactured & 1222 \\
\hline 2. Coca-cola & Flavored waters, non alcoholic (5 digit) under Beverage non-alcohol nes & 11102 \\
\hline 3. Cognac & Brandies/marc, etc (5 digit) under Distilled Alcoholic beverages & 11242 \\
\hline 4. Gin & Gin/Geneva (5 digit) under Distilled alcoholic beverages & 11245 \\
\hline 5. Time magazine & Newspapers/periodic nes (5 digit) under Newspapers/periodicals & 89229 \\
\hline 6. Color Film & Photo film roll unexposed (4 digit) under Photographic supplies & 8823 \\
\hline 7. Cointreau & Brandies/marc, etc ( 5 digit) under Distilled Alcoholic beverages & 11242 \\
\hline 8. Vermouth & Vermouth/flavored wine ( 5 digit) under Wines of fresh grapes & 11213 \\
\hline
\end{tabular}


Table 1: Pass-through to Imported Good Prices

\begin{tabular}{|c|c|c|c|c|c|c|c|c|c|c|}
\hline change in & exchange rate & $\begin{array}{c}\text { Eqn 1 } \\
0.423 \text { *** } \\
(0.029)\end{array}$ & $\begin{array}{c}\text { Eqn2 } \\
0.758 \text { *** } \\
(0.063)\end{array}$ & $\begin{array}{c}\text { Eqn3 } \\
0.690 \text { *** } \\
(0.065)\end{array}$ & $\begin{array}{r}\text { Eqn 4 } \\
1.215 \\
(0.922)\end{array}$ & $\begin{array}{r}\text { Eqn 5 } \\
1.350 \\
(0.921)\end{array}$ & $\begin{array}{c}\text { Eqn 6 } \\
1.794 \text { ** } \\
(0.878)\end{array}$ & $\begin{array}{r}\text { Eqn } 7 \\
1.076 \\
(0.812)\end{array}$ & $\begin{array}{r}\text { Eqn 8 } \\
1.168 \\
(0.822)\end{array}$ & $\begin{array}{r}\text { Eqn 9 } \\
1.353 \\
(0.848)\end{array}$ \\
\hline change in & exporter's price & $\begin{array}{l}0.086 * * * \\
(0.024)\end{array}$ & $\begin{array}{l}0.100 * * * \\
(0.024)\end{array}$ & $\begin{array}{l}0.103^{* * *} \\
(0.024)\end{array}$ & $\begin{array}{l}0.1022^{* * *} \\
(0.025)\end{array}$ & $\begin{array}{l}0.1011^{* * * *} \\
(0.025)\end{array}$ & $\begin{array}{l}0.066 * * \\
(0.029)\end{array}$ & $\begin{array}{l}0.050 * \\
(0.029)\end{array}$ & $\begin{array}{l}0.050 * \\
(0.029)\end{array}$ & $\begin{array}{l}0.060 * * \\
(0.028)\end{array}$ \\
\hline$($ delta s)* & trend & & $\begin{array}{l}-0.053 * * * \\
(0.008)\end{array}$ & $\begin{array}{l}-0.053^{* * *} \\
(0.008)\end{array}$ & $\begin{array}{l}-0.052^{* * *} \\
(0.008)\end{array}$ & $\begin{array}{l}-0.052^{* * *} \\
(0.008)\end{array}$ & $\begin{array}{l}-0.072^{* * *} \\
(0.010)\end{array}$ & $\begin{array}{l}-0.050^{* * *} \\
(0.010)\end{array}$ & $\begin{array}{l}-0.050 * * * \\
(0.010)\end{array}$ & $\begin{array}{l}-0.048^{* * *} \\
(0.010)\end{array}$ \\
\hline$($ delta s)* & $\begin{array}{l}\log [\text { per capitaRGDP(importer) / } \\
\text { per capitaRGDP(exporter)] }\end{array}$ & & & $\begin{array}{l}-0.035^{* * *} \\
(0.009)\end{array}$ & $\begin{array}{l}-0.039^{* * *} \\
(0.010)\end{array}$ & $\begin{array}{l}-0.057 * * * \\
(0.017)\end{array}$ & $\begin{array}{l}-0.072^{* * *} \\
(0.022)\end{array}$ & $\begin{array}{l}-0.044 * \\
(0.023)\end{array}$ & $\begin{array}{l}-0.047^{* *} \\
(0.024)\end{array}$ & $\begin{array}{l}-0.058 * * \\
(0.023)\end{array}$ \\
\hline$($ delta s)* & tariff levels & & & & $\begin{array}{r}-0.085 \\
(0.174)\end{array}$ & $\begin{array}{r}-0.116 \\
(0.174)\end{array}$ & $\begin{array}{r}-0.170 \\
(0.148)\end{array}$ & $\begin{array}{r}-0.066 \\
(0.142)\end{array}$ & $\begin{array}{r}-0.082 \\
(0.144)\end{array}$ & $\begin{array}{r}-0.109 \\
(0.143)\end{array}$ \\
\hline$($ delta s)* & $\log$ distance & & & & $\begin{array}{r}-0.014 \\
(0.039)\end{array}$ & $\begin{array}{r}-0.007 \\
(0.040)\end{array}$ & $\begin{array}{r}-0.013 \\
(0.051)\end{array}$ & $\begin{array}{r}-0.013 \\
(0.050)\end{array}$ & $\begin{array}{r}-0.013 \\
(0.050)\end{array}$ & $\begin{array}{r}-0.027 \\
(0.050)\end{array}$ \\
\hline$($ delta s)* & $\log [$ RGDP(importer)/RGDP(exporter)] & & & & & $\begin{array}{r}0.019 \\
(0.015)\end{array}$ & $\begin{array}{c}0.038 * \\
(0.020)\end{array}$ & $\begin{array}{r}0.019 \\
(0.020)\end{array}$ & $\begin{array}{r}0.024 \\
(0.021)\end{array}$ & $\begin{array}{r}0.031 \\
(0.020)\end{array}$ \\
\hline$($ delta s)* & log real wage $(\$)$ & & & & & & $\begin{array}{r}-0.006 \\
(0.008)\end{array}$ & $\begin{array}{r}-0.005 \\
(0.008)\end{array}$ & $\begin{array}{r}-0.005 \\
(0.008)\end{array}$ & $\begin{array}{r}-0.005 \\
(0.007)\end{array}$ \\
\hline$($ delta s)* & long term inflation & & & & & & & $\begin{array}{c}0.353 * \\
(0.202)\end{array}$ & $\begin{array}{c}0.358 * \\
(0.202)\end{array}$ & $\begin{array}{l}0.237^{* * *} \\
(0.067)\end{array}$ \\
\hline$(\text { delta } \mathrm{s})^{*}$ & long term exchange rate variability & & & & & & & $\begin{array}{r}-1.211 \\
(1.292)\end{array}$ & $\begin{array}{r}-1.261 \\
(1.295)\end{array}$ & \\
\hline$($ delta s)* & US Importer dummy & & & & & & & & $\begin{array}{l}-0.365^{* *} \\
(0.162)\end{array}$ & $\begin{array}{l}-0.4411^{* * *} \\
(0.162)\end{array}$ \\
\hline & Error Correction term (ECM) & $\begin{array}{l}-0.107^{* * *} \\
(0.007)\end{array}$ & $\begin{array}{l}-0.159 \text { *** } \\
(0.016)\end{array}$ & $\begin{array}{l}-0.158^{\text {**** }} \\
(0.015)\end{array}$ & $\begin{array}{l}-0.436^{* * *} \\
(0.105)\end{array}$ & $\begin{array}{l}-0.458 * * * \\
(0.105)\end{array}$ & $\begin{array}{l}-0.602^{* * *} \\
(0.116)\end{array}$ & $\begin{array}{l}-0.385^{* * * *} \\
(0.116)\end{array}$ & $\begin{array}{l}-0.383^{* * *} \\
(0.116)\end{array}$ & $\begin{array}{l}-0.465^{* * *} \\
(0.118)\end{array}$ \\
\hline $\mathrm{ECM}^{*}$ & trend & & $\begin{array}{l}0.007^{* * *} \\
(0.002)\end{array}$ & $\begin{array}{l}0.007 \text { *** } \\
(0.002)\end{array}$ & $\begin{array}{l}0.008^{* * *} \\
(0.002)\end{array}$ & $\begin{array}{l}0.008^{* * *} \\
(0.002)\end{array}$ & $\begin{array}{l}0.006^{* * *} \\
(0.002)\end{array}$ & $\begin{array}{c}0.004 * \\
(0.002)\end{array}$ & $\begin{array}{l}0.004 * \\
(0.002)\end{array}$ & $\begin{array}{l}0.004 * \\
(0.002)\end{array}$ \\
\hline $\mathrm{ECM}^{*}$ & $\begin{array}{l}\log [\text { per capitaRGDP(importer) / } \\
\text { per capitaRGDP(exporter)] }\end{array}$ & & & $\begin{array}{r}0.001 \\
(0.002)\end{array}$ & $\begin{array}{r}0.003 \\
(0.003)\end{array}$ & $\begin{array}{l}0.006 * \\
(0.004)\end{array}$ & $\begin{array}{r}-0.001 \\
(0.006)\end{array}$ & $\begin{array}{r}0.000 \\
(0.006)\end{array}$ & $\begin{array}{r}-0.001 \\
(0.006)\end{array}$ & $\begin{array}{r}-0.002 \\
(0.006)\end{array}$ \\
\hline $\mathrm{ECM}^{*}$ & tariff levels & & & & $\begin{array}{r}0.021 \\
(0.017)\end{array}$ & $\begin{array}{r}0.025 \\
(0.018)\end{array}$ & $\begin{array}{r}0.034 \\
(0.022)\end{array}$ & $\begin{array}{r}0.006 \\
(0.021)\end{array}$ & $\begin{array}{r}0.006 \\
(0.021)\end{array}$ & $\begin{array}{r}0.012 \\
(0.022)\end{array}$ \\
\hline $\mathrm{ECM}^{*}$ & log distance & & & & $\begin{array}{l}0.018^{* *} \\
(0.008)\end{array}$ & $\begin{array}{l}0.018 * * \\
(0.009)\end{array}$ & $\begin{array}{l}0.040^{* * *} \\
(0.010)\end{array}$ & $\begin{array}{l}0.029^{* * *} \\
(0.011)^{*}\end{array}$ & $\begin{array}{l}0.030^{* * *} \\
(0.011)\end{array}$ & $\begin{array}{l}0.036^{* * *} \\
(0.010)\end{array}$ \\
\hline ECM* & $\log [$ RGDP(importer)/RGDP(exporter) $]$ & & & & & $\begin{array}{r}-0.003 \\
(0.004)\end{array}$ & $\begin{array}{r}0.001 \\
(0.006)\end{array}$ & $\begin{array}{r}0.000 \\
(0.005)\end{array}$ & $\begin{array}{r}0.001 \\
(0.006)\end{array}$ & $\begin{array}{r}0.003 \\
(0.006)\end{array}$ \\
\hline $\mathrm{ECM}^{*}$ & log real wage $(\$)$ & & & & & & $\begin{array}{l}0.017 * * \\
(0.007)\end{array}$ & $\begin{array}{r}0.001 \\
(0.008)\end{array}$ & $\begin{array}{r}0.001 \\
(0.008)\end{array}$ & $\begin{array}{r}0.006 \\
(0.008)\end{array}$ \\
\hline ECM* & long term inflation & & & & & & & $\begin{array}{l}-0.083 * \\
(0.046)\end{array}$ & $\begin{array}{l}-0.083 * \\
(0.046)\end{array}$ & $\begin{array}{l}-0.092 * * \\
(0.045)\end{array}$ \\
\hline $\mathrm{ECM}^{*}$ & long term exchange rate variability & & & & & & & $\begin{array}{l}-0.185 * * \\
(0.072)\end{array}$ & $\begin{array}{l}-0.183 * * \\
(0.072)\end{array}$ & \\
\hline $\mathrm{ECM}^{*}$ & US Importer dummy & & & & & & & & $\begin{array}{r}-0.057 \\
(0.062)\end{array}$ & $\begin{array}{r}-0.062 \\
(0.063)\end{array}$ \\
\hline & \# of Observations & 5677 & 5677 & 5677 & 5192 & 5192 & 3316 & 3250 & 3250 & 3316 \\
\hline & Adjusted R-squared & 0.297 & 0.315 & 0.321 & 0.330 & 0.330 & 0.316 & 0.288 & 0.288 & 0.330 \\
\hline & Country dummies & yes & yes & yes & yes & yes & yes & yes & yes & yes \\
\hline & Product dummies & yes & yes & yes & yes & yes & yes & yes & yes & yes \\
\hline
\end{tabular}

Full sample

$*=10 \%, * *=5 \%$, and $* * *=1 \%$ levels of significance

product specific (ps) tariffs 
Table 2: Pass-through to Local Competitor Prices

\begin{tabular}{|c|c|c|c|c|c|c|c|c|c|c|}
\hline & & Eqn 1 & Eqn2 & Eqn3 & Eqn 4 & Eqn 5 & Eqn 6 & Eqn 7 & Eqn 8 & Eqn 9 \\
\hline change in & exchange rate & $\begin{array}{l}0.396^{* * *} \\
(0.027)\end{array}$ & $\begin{array}{l}0.756^{* * *} \\
(0.053)\end{array}$ & $\begin{array}{l}0.723^{* * *} \\
(0.054)\end{array}$ & $\begin{array}{r}-0.382 \\
(0.550)\end{array}$ & $\begin{array}{r}-0.017 \\
(0.563)\end{array}$ & $\begin{array}{r}-0.668 \\
(0.786)\end{array}$ & $\begin{array}{r}-1.015 \\
(0.791)\end{array}$ & $\begin{array}{r}-1.117 \\
(0.800)\end{array}$ & $\begin{array}{r}-1.142 \\
(0.832)\end{array}$ \\
\hline change in & exporter's price & $\begin{array}{l}0.052 * \\
(0.027)\end{array}$ & $\begin{array}{r}0.038 \\
(0.028)\end{array}$ & $\begin{array}{r}0.040 \\
(0.028)\end{array}$ & $\begin{array}{r}0.022 \\
(0.030)\end{array}$ & $\begin{array}{r}0.019 \\
(0.030)\end{array}$ & $\begin{array}{r}0.014 \\
(0.037)\end{array}$ & $\begin{array}{r}0.013 \\
(0.035)\end{array}$ & $\begin{array}{r}0.013 \\
(0.035)\end{array}$ & $\begin{array}{r}0.009 \\
(0.037)\end{array}$ \\
\hline$($ delta s)* & trend & & $\begin{array}{l}-0.057^{* * *} \\
(0.007)^{2}\end{array}$ & $\begin{array}{l}-0.057^{* * *} \\
(0.007)\end{array}$ & $\begin{array}{l}-0.057^{* * *} \\
(0.007)\end{array}$ & $\begin{array}{l}-0.058^{* * *} \\
(0.007)\end{array}$ & $\begin{array}{l}-0.082^{* * *} \\
(0.009)\end{array}$ & $\begin{array}{l}-0.050^{* * *} \\
(0.010)\end{array}$ & $\begin{array}{l}-0.050 * * * \\
(0.010)\end{array}$ & $\begin{array}{l}-0.0633^{* * *} \\
(0.011)\end{array}$ \\
\hline$($ delta s)* & $\begin{array}{l}\log [\text { per capitaRGDP(importer) / } \\
\text { per capitaRGDP(exporter)] }\end{array}$ & & & $\begin{array}{l}-0.018^{* * *} \\
(0.006)\end{array}$ & $\begin{array}{l}-0.020 * * * \\
(0.006)\end{array}$ & $\begin{array}{l}-0.061^{* * *} \\
(0.016)\end{array}$ & $\begin{array}{l}-0.094^{* * *} \\
(0.025)\end{array}$ & $\begin{array}{l}-0.113^{* * *} \\
(0.023)\end{array}$ & $\begin{array}{l}-0.109 * * * \\
(0.023)\end{array}$ & $\begin{array}{l}-0.078 * * * \\
(0.026)\end{array}$ \\
\hline$($ delta s)* & tariff levels & & & & $\begin{array}{r}0.011 \\
(0.093)\end{array}$ & $\begin{array}{r}-0.075 \\
(0.097)\end{array}$ & $\begin{array}{r}-0.010 \\
(0.133)\end{array}$ & $\begin{array}{r}0.126 \\
(0.133)\end{array}$ & $\begin{array}{r}0.144 \\
(0.135)\end{array}$ & $\begin{array}{r}0.053 \\
(0.144)\end{array}$ \\
\hline$($ delta s)* & $\log$ distance & & & & $\begin{array}{l}0.118^{* * *} \\
(0.039)\end{array}$ & $\begin{array}{l}0.134^{* * *} \\
(0.039)\end{array}$ & $\begin{array}{l}0.193 \text { *** } \\
(0.054)\end{array}$ & $\begin{array}{l}0.139^{* * *} \\
(0.049)^{* *}\end{array}$ & $\begin{array}{l}0.139 \text { *** } \\
(0.049)\end{array}$ & $\begin{array}{l}0.186 \text { *** } \\
(0.055)\end{array}$ \\
\hline$($ delta s)* & $\log [$ RGDP(importer)/RGDP(exporter) $]$ & & & & & $\begin{array}{l}0.042 \text { *** } \\
(0.015)\end{array}$ & $\begin{array}{l}0.078^{\text {*** }} \\
(0.022)\end{array}$ & $\begin{array}{l}0.099^{\text {**** }} \\
(0.021)^{\text {n }}\end{array}$ & $\begin{array}{l}0.093 \text { *** } \\
(0.022)\end{array}$ & $\begin{array}{l}0.065^{* * *} \\
(0.023)\end{array}$ \\
\hline$($ delta s)* & log real wage $(\$)$ & & & & & & $\begin{array}{l}-0.019 \text { ** } \\
(0.008)\end{array}$ & $\begin{array}{r}-0.010 \\
(0.007)\end{array}$ & $\begin{array}{r}-0.010 \\
(0.007)\end{array}$ & $\begin{array}{l}-0.018 * * \\
(0.008)\end{array}$ \\
\hline$($ delta s)* & long term inflation & & & & & & & $\begin{array}{l}0.6211^{* *} \\
(0.254)\end{array}$ & $\begin{array}{l}0.615 \text { ** } \\
(0.254)\end{array}$ & $\begin{array}{l}0.180 \text { *** } \\
(0.063)\end{array}$ \\
\hline$($ delta s)* & long term exchange rate variability & & & & & & & $\begin{array}{r}-2.222 \\
(1.640)\end{array}$ & $\begin{array}{r}-2.166 \\
(1.643)\end{array}$ & \\
\hline$($ delta s)* & US Importer dummy & & & & & & & & $\begin{array}{l}0.436 \text { ** } \\
(0.182)\end{array}$ & $\begin{array}{l}0.478 \text { *** } \\
(0.184)\end{array}$ \\
\hline & Error Correction term (ECM) & $\begin{array}{l}-0.018^{* * *} \\
(0.006)\end{array}$ & $\begin{array}{r}0.006 \\
(0.014)\end{array}$ & $\begin{array}{r}0.005 \\
(0.014)\end{array}$ & $\begin{array}{r}0.023 \\
(0.084)\end{array}$ & $\begin{array}{r}0.047 \\
(0.084)\end{array}$ & $\begin{array}{r}-0.033 \\
(0.113)\end{array}$ & $\begin{array}{r}-0.038 \\
(0.111)\end{array}$ & $\begin{array}{r}-0.037 \\
(0.111)\end{array}$ & $\begin{array}{r}-0.004 \\
(0.113)\end{array}$ \\
\hline $\mathrm{ECM}^{*}$ & trend & & $\begin{array}{l}-0.003 \text { ** } \\
(0.001)\end{array}$ & $\begin{array}{l}-0.003 \text { ** } \\
(0.001)\end{array}$ & $\begin{array}{l}-0.003 * * \\
(0.002)\end{array}$ & $\begin{array}{l}-0.003 * * \\
(0.002)\end{array}$ & $\begin{array}{r}-0.001 \\
(0.002)\end{array}$ & $\begin{array}{r}-0.002 \\
(0.002)\end{array}$ & $\begin{array}{r}-0.002 \\
(0.002)\end{array}$ & $\begin{array}{r}-0.001 \\
(0.002)\end{array}$ \\
\hline $\mathrm{ECM}^{*}$ & $\begin{array}{l}\log [\text { per capitaRGDP(importer) / } \\
\text { per capitaRGDP(exporter)] }\end{array}$ & & & $\begin{array}{r}-0.001 \\
(0.002)\end{array}$ & $\begin{array}{r}-0.002 \\
(0.002)\end{array}$ & $\begin{array}{l}-0.008 * * \\
(0.004)\end{array}$ & $\begin{array}{l}-0.020^{* * *} \\
(0.006)\end{array}$ & $\begin{array}{l}-0.016^{* * *} \\
(0.006)\end{array}$ & $\begin{array}{l}-0.016^{* * *} \\
(0.006)\end{array}$ & $\begin{array}{l}-0.020 * * * \\
(0.006)\end{array}$ \\
\hline $\mathrm{ECM}^{*}$ & tariff levels & & & & $\begin{array}{r}-0.009 \\
(0.012)\end{array}$ & $\begin{array}{r}-0.012 \\
(0.012)\end{array}$ & $\begin{array}{r}-0.025 \\
(0.020)\end{array}$ & $\begin{array}{r}-0.026 \\
(0.019)\end{array}$ & $\begin{array}{r}-0.026 \\
(0.019)\end{array}$ & $\begin{array}{r}-0.030 \\
(0.020)\end{array}$ \\
\hline $\mathrm{ECM}^{*}$ & $\log$ distance & & & & $\begin{array}{r}0.003 \\
(0.007)\end{array}$ & $\begin{array}{r}0.003 \\
(0.007)\end{array}$ & $\begin{array}{l}0.027^{* * *} \\
(0.008)\end{array}$ & $\begin{array}{l}0.027 \text { *** } \\
(0.009)\end{array}$ & $\begin{array}{l}0.027^{* * *} \\
(0.009)\end{array}$ & $\begin{array}{l}0.027^{* * *} \\
(0.008)\end{array}$ \\
\hline ECM* & $\log [$ RGDP(importer)/RGDP(exporter) $]$ & & & & & $\begin{array}{l}0.006 * \\
(0.003)\end{array}$ & $\begin{array}{l}0.015^{* * *} \\
(0.005)\end{array}$ & $\begin{array}{l}0.011 * * \\
(0.005)\end{array}$ & $\begin{array}{l}0.011 * * \\
(0.005)\end{array}$ & $\begin{array}{l}0.015^{* * *} \\
(0.005)\end{array}$ \\
\hline $\mathrm{ECM}^{*}$ & log real wage $(\$)$ & & & & & & $\begin{array}{l}0.019^{* * *} \\
(0.007)^{*}\end{array}$ & $\begin{array}{c}0.013 * \\
(0.007)\end{array}$ & $\begin{array}{c}0.013 * \\
(0.007)\end{array}$ & $\begin{array}{l}0.018 \text { ** } \\
(0.007)\end{array}$ \\
\hline ECM* & long term inflation & & & & & & & $\begin{array}{r}0.011 \\
(0.025)\end{array}$ & $\begin{array}{r}0.011 \\
(0.025)\end{array}$ & $\begin{array}{r}-0.005 \\
(0.026)\end{array}$ \\
\hline $\mathrm{ECM}^{*}$ & long term exchange rate variability & & & & & & & $\begin{array}{l}-0.175 * * \\
(0.077)\end{array}$ & $\begin{array}{l}-0.176 * * \\
(0.077)\end{array}$ & \\
\hline ECM* & US Importer dummy & & & & & & & & $\begin{array}{r}-0.018 \\
(0.053)\end{array}$ & $\begin{array}{r}-0.041 \\
(0.053)\end{array}$ \\
\hline & \# of Observations & 5630 & 5630 & 5630 & 5077 & 5077 & 3299 & 3235 & 3235 & 3299 \\
\hline & Adjusted R-squared & 0.224 & 0.240 & 0.242 & 0.248 & 0.250 & 0.241 & 0.259 & 0.259 & 0.246 \\
\hline & Country dummies & yes & yes & yes & yes & yes & yes & yes & yes & yes \\
\hline & Product dummies & yes & yes & yes & yes & yes & yes & yes & yes & yes \\
\hline
\end{tabular}

Full sample

Product dummies

$*=10 \%, * *=5 \%$, and $* * *=1 \%$ levels of significance

product specific (ps) tariffs 
Table 3: Pass-through to the CPI

\begin{tabular}{|c|c|c|c|c|c|c|c|c|}
\hline change in & exchange rate & $\begin{array}{c}\text { Eqn 1 } \\
0.279 * * * \\
(0.052)\end{array}$ & $\begin{array}{c}\text { Eqn2 } \\
0.602 \\
(0.103)\end{array}$ & $\begin{array}{c}\text { Eqn3 } \\
0.623 \\
(0.135)\end{array}$ & $\begin{array}{c}\text { Eqn } 4 \\
3.254 * * \\
(1.641)\end{array}$ & $\begin{array}{c}\text { Eqn 5 } \\
4.898 \text { ** } \\
(2.061)\end{array}$ & $\begin{array}{c}\text { Eqn 6 } \\
5.621 \\
(1.840)\end{array}$ & $\begin{array}{c}\text { Eqn } 7 \\
2.616 \text { ** } \\
(1.331)\end{array}$ \\
\hline$($ delta s)* & trend & & $\begin{array}{l}-0.054^{* * *} \\
(0.011)\end{array}$ & $\begin{array}{l}-0.055^{* * *} \\
(0.011)\end{array}$ & $\begin{array}{l}-0.054^{* * *} \\
(0.012)\end{array}$ & $\begin{array}{l}-0.057 * * * \\
(0.012)\end{array}$ & $\begin{array}{l}-0.066^{* * *} \\
(0.011)\end{array}$ & $\begin{array}{r}-0.011 \\
(0.007)\end{array}$ \\
\hline$($ delta s)* & $\begin{array}{l}\log [\text { per capitaRGDP(importer) / } \\
\text { per capitaRGDP(exporter)] }\end{array}$ & & & $\begin{array}{r}0.009 \\
(0.040)\end{array}$ & $\begin{array}{r}-0.037 \\
(0.041)\end{array}$ & $\begin{array}{l}-0.099 * \\
(0.052)\end{array}$ & $\begin{array}{l}0.777^{* * *} \\
(0.195)^{*}\end{array}$ & $\begin{array}{l}0.466 * * \\
(0.100)\end{array}$ \\
\hline$($ delta s)* & tariff levels & & & & $\begin{array}{r}-0.509 \\
(0.356)\end{array}$ & $\begin{array}{l}-0.860 * * \\
(0.438)\end{array}$ & $\begin{array}{l}-0.934 * * \\
(0.446)\end{array}$ & $\begin{array}{r}-0.287 \\
(0.306)\end{array}$ \\
\hline$($ delta s)* & $\log$ distance & & & & $\begin{array}{r}-0.031 \\
(0.055)\end{array}$ & $\begin{array}{r}-0.001 \\
(0.074)\end{array}$ & $\begin{array}{r}-0.157 \\
(0.131)\end{array}$ & $\begin{array}{l}-0.156 * * \\
(0.069)\end{array}$ \\
\hline$($ delta s)* & $\log [$ RGDP(importer)/RGDP(exporter)] & & & & & $\begin{array}{l}0.084 * \\
(0.043)\end{array}$ & $\begin{array}{r}0.034 \\
(0.034)\end{array}$ & $\begin{array}{l}0.055 * * \\
(0.020)\end{array}$ \\
\hline$($ delta s)* & log real wage $(\$)$ & & & & & & $\begin{array}{l}-0.540^{* * *} \\
(0.077)^{*}\end{array}$ & $\begin{array}{l}-0.334 * * \\
(0.050)\end{array}$ \\
\hline$($ delta s)* & long term inflation & & & & & & & $\begin{array}{l}0.907 * * \\
(0.139)\end{array}$ \\
\hline$($ delta s)* & long term exchange rate variability & & & & & & & $\begin{array}{l}-4.303 * * \\
(0.929)\end{array}$ \\
\hline & Error Correction term (ECM) & $\begin{array}{r}-0.014 \\
(0.009)\end{array}$ & $\begin{array}{r}-0.003 \\
(0.006)\end{array}$ & $\begin{array}{r}-0.003 \\
(0.006)\end{array}$ & $\begin{array}{r}-0.417 \\
(0.319)\end{array}$ & $\begin{array}{r}-0.428 \\
(0.307)\end{array}$ & $\begin{array}{l}-0.900 * \\
(0.477)\end{array}$ & $\begin{array}{l}0.461 * * \\
(0.189)\end{array}$ \\
\hline $\mathrm{ECM}^{*}$ & trend & & $\begin{array}{l}0.003^{* * *} \\
(0.001)^{2}\end{array}$ & $\begin{array}{l}0.004^{* * *} \\
(0.001)\end{array}$ & $\begin{array}{l}0.008^{* * *} \\
(0.002)\end{array}$ & $\begin{array}{l}0.008^{* * *} \\
(0.002)\end{array}$ & $\begin{array}{l}0.010^{* * *} \\
(0.003)\end{array}$ & $\begin{array}{l}0.006 * * \\
(0.002)\end{array}$ \\
\hline $\mathrm{ECM}^{*}$ & $\begin{array}{l}\log [\text { per capitaRGDP(importer) / } \\
\text { per capitaRGDP(exporter)] }\end{array}$ & & & $\begin{array}{r}0.015 \\
(0.011)\end{array}$ & $\begin{array}{l}0.014 * \\
(0.008)\end{array}$ & $\begin{array}{r}0.010 \\
(0.008)\end{array}$ & $\begin{array}{r}0.012 \\
(0.055)\end{array}$ & $\begin{array}{r}-0.056 \\
(0.039)\end{array}$ \\
\hline $\mathrm{ECM}^{*}$ & tariff levels & & & & $\begin{array}{r}0.089 \\
(0.067)\end{array}$ & $\begin{array}{r}0.091 \\
(0.065)\end{array}$ & $\begin{array}{l}0.194 * \\
(0.102)\end{array}$ & $\begin{array}{l}-0.099 * * \\
(0.040)\end{array}$ \\
\hline $\mathrm{ECM}^{*}$ & $\log$ distance & & & & $\begin{array}{l}-0.006^{* * *} \\
(0.002)\end{array}$ & $\begin{array}{r}-0.004 \\
(0.004)\end{array}$ & $\begin{array}{l}-0.011 * \\
(0.006)\end{array}$ & $\begin{array}{r}0.002 \\
(0.004)\end{array}$ \\
\hline $\mathrm{ECM}^{*}$ & $\log [$ RGDP(importer)/RGDP(exporter)] & & & & & $\begin{array}{r}0.005 \\
(0.007)\end{array}$ & $\begin{array}{r}0.003 \\
(0.008)\end{array}$ & $\begin{array}{r}0.003 \\
(0.006)\end{array}$ \\
\hline $\mathrm{ECM}^{*}$ & log real wage $(\$)$ & & & & & & $\begin{array}{r}-0.003 \\
(0.029)\end{array}$ & $\begin{array}{l}0.040 * \\
(0.021)\end{array}$ \\
\hline $\mathrm{ECM}^{*}$ & long term inflation & & & & & & & $\begin{array}{l}-0.162 * * \\
(0.039)\end{array}$ \\
\hline $\mathrm{ECM}^{*}$ & long term exchange rate variability & & & & & & & $\begin{array}{r}0.115 * \\
(0.065)\end{array}$ \\
\hline & \# of Observations & 732 & 732 & 732 & 732 & 732 & 732 & 732 \\
\hline & Adjusted R-squared & 0.693 & 0.693 & 0.693 & 0.693 & 0.693 & 0.693 & 0.693 \\
\hline & Country dummies & yes & yes & yes & yes & yes & yes & yes \\
\hline
\end{tabular}

full sample

Average Tariffs

$*=10 \%, * *=5 \%$, and $* * *=1 \%$ levels of significance 
Table 4: Pass-through to Unit Values

\begin{tabular}{|c|c|c|c|c|c|c|c|c|c|c|}
\hline change in & exchange rate & $\begin{array}{c}\text { Eqn 1 } \\
0.6666^{* * *} \\
(0.067)\end{array}$ & $\begin{array}{c}\text { Eqn2 } \\
0.423 \\
(0.144)\end{array}$ & $\begin{array}{c}\text { Eqn3 } \\
0.451 \text { *** } \\
(0.143)\end{array}$ & $\begin{array}{c}\operatorname{Eqn~} 4_{5.339}^{* * *} \\
(1.265)\end{array}$ & $\begin{array}{c}\text { Eqn 5 } \\
5.1699^{* * *} \\
(1.367)\end{array}$ & $\begin{array}{r}\text { Eqn 6 } \\
1.966 \\
(1.520)\end{array}$ & $\begin{array}{r}\text { Eqn } 7 \\
1.663 \\
(1.508)\end{array}$ & $\begin{array}{r}\text { Eqn 8 } \\
1.697 \\
(1.537)\end{array}$ & $\begin{array}{r}\text { Eqn 9 } \\
2.195 \\
(1.572)\end{array}$ \\
\hline change in & exporter's price & $\begin{array}{r}0.094 \\
(0.063)\end{array}$ & $\begin{array}{l}0.136 * * \\
(0.065)\end{array}$ & $\begin{array}{l}0.138 * * \\
(0.065)\end{array}$ & $\begin{array}{l}0.137 * * \\
(0.068)\end{array}$ & $\begin{array}{l}0.135 * * \\
(0.068)\end{array}$ & $\begin{array}{l}0.263^{* * *} \\
(0.072)\end{array}$ & $\begin{array}{l}0.258 \text { *** } \\
(0.073)\end{array}$ & $\begin{array}{l}0.257^{* * *} \\
(0.073)\end{array}$ & $\begin{array}{l}0.271^{* * *} \\
(0.072)\end{array}$ \\
\hline$($ delta s)* & trend & & $\begin{array}{l}0.037 * * \\
(0.017)\end{array}$ & $\begin{array}{l}0.039 * * \\
(0.017)\end{array}$ & $\begin{array}{l}0.041 * * \\
(0.017)\end{array}$ & $\begin{array}{l}0.042 * * \\
(0.018)\end{array}$ & $\begin{array}{l}0.049 * * \\
(0.021)\end{array}$ & $\begin{array}{l}0.040 * \\
(0.024)\end{array}$ & $\begin{array}{l}0.040 * \\
(0.024)\end{array}$ & $\begin{array}{r}0.033 \\
(0.024)\end{array}$ \\
\hline$($ delta s)* & $\begin{array}{l}\log [\text { per capitaRGDP(importer) / } \\
\text { per capitaRGDP(exporter)] }\end{array}$ & & & $\begin{array}{l}0.028 * \\
(0.015)\end{array}$ & $\begin{array}{l}0.028 * * \\
(0.014)\end{array}$ & $\begin{array}{r}0.040 \\
(0.041)\end{array}$ & $\begin{array}{l}0.105 * * \\
(0.051)\end{array}$ & $\begin{array}{l}0.151^{* * *} \\
(0.055)\end{array}$ & $\begin{array}{l}0.149^{* * *} \\
(0.055)\end{array}$ & $\begin{array}{l}0.100 * \\
(0.053)\end{array}$ \\
\hline$($ delta s)* & tariff levels & & & & $\begin{array}{l}-0.482 * * \\
(0.235)\end{array}$ & $\begin{array}{l}-0.448 * \\
(0.255)\end{array}$ & $\begin{array}{r}-0.066 \\
(0.296)\end{array}$ & $\begin{array}{r}-0.040 \\
(0.299)\end{array}$ & $\begin{array}{r}-0.046 \\
(0.304)\end{array}$ & $\begin{array}{r}-0.084 \\
(0.301)\end{array}$ \\
\hline$($ delta s)* & $\log$ distance & & & & $\begin{array}{l}-0.290 \text { *** } \\
(0.074)\end{array}$ & $\begin{array}{l}-0.292 \text { *** } \\
(0.074)\end{array}$ & $\begin{array}{l}-0.163 * * \\
(0.083)\end{array}$ & $\begin{array}{r}-0.141 \\
(0.093)\end{array}$ & $\begin{array}{r}-0.141 \\
(0.093)\end{array}$ & $\begin{array}{l}-0.159 * \\
(0.082)\end{array}$ \\
\hline$($ delta s)* & $\log [$ RGDP(importer)/RGDP(exporter)] & & & & & $\begin{array}{r}-0.013 \\
(0.040)\end{array}$ & $\begin{array}{l}-0.116 * * \\
(0.047)\end{array}$ & $\begin{array}{l}-0.156^{* * *} \\
(0.047)\end{array}$ & $\begin{array}{l}-0.154 * * * \\
(0.049)\end{array}$ & $\begin{array}{l}-0.115 * * \\
(0.049)\end{array}$ \\
\hline$($ delta s)* & log real wage $(\$)$ & & & & & & $\begin{array}{r}-0.002 \\
(0.013)\end{array}$ & $\begin{array}{r}-0.020 \\
(0.016)\end{array}$ & $\begin{array}{r}-0.020 \\
(0.016)\end{array}$ & $\begin{array}{r}-0.005 \\
(0.013)\end{array}$ \\
\hline$($ delta s)* & long term inflation & & & & & & & $\begin{array}{r}-0.553 \\
(0.404)\end{array}$ & $\begin{array}{r}-0.551 \\
(0.404)\end{array}$ & $\begin{array}{r}-0.337 \\
(0.223)\end{array}$ \\
\hline$($ delta s)* & long term exchange rate variability & & & & & & & $\begin{array}{r}1.350 \\
(2.202)\end{array}$ & $\begin{array}{r}1.330 \\
(2.214)\end{array}$ & \\
\hline$($ delta s)* & US Importer dummy & & & & & & & & $\begin{array}{r}-0.140 \\
(0.278)\end{array}$ & $\begin{array}{r}-0.169 \\
(0.269)\end{array}$ \\
\hline & Error Correction term (ECM) & $\begin{array}{l}-0.218 * * * \\
(0.030)\end{array}$ & $\begin{array}{l}-0.183^{* * *} \\
(0.033)\end{array}$ & $\begin{array}{l}-0.186^{* * *} \\
(0.034)\end{array}$ & $\begin{array}{r}0.064 \\
(0.121)\end{array}$ & $\begin{array}{r}0.091 \\
(0.135)\end{array}$ & $\begin{array}{c}0.266 * \\
(0.148)\end{array}$ & $\begin{array}{l}0.388 \text { *** } \\
(0.148)\end{array}$ & $\begin{array}{l}0.388 \text { *** } \\
(0.148)\end{array}$ & $\begin{array}{c}0.283 * \\
(0.149)\end{array}$ \\
\hline $\mathrm{ECM}^{*}$ & trend & & $\begin{array}{l}-0.005 * * \\
(0.002)\end{array}$ & $\begin{array}{l}-0.005 * * \\
(0.002)\end{array}$ & $\begin{array}{l}-0.005 \text { ** } \\
(0.002)\end{array}$ & $\begin{array}{l}-0.005 * * \\
(0.002)\end{array}$ & $\begin{array}{l}-0.006 * \\
(0.003)\end{array}$ & $\begin{array}{l}-0.007 \text { ** } \\
(0.003)\end{array}$ & $\begin{array}{l}-0.007 * * \\
(0.003)\end{array}$ & $\begin{array}{l}-0.006 \text { ** } \\
(0.003)\end{array}$ \\
\hline $\mathrm{ECM}^{*}$ & $\begin{array}{l}\log [\text { per capitaRGDP(importer) } / \\
\text { per capitaRGDP(exporter)] }\end{array}$ & & & $\begin{array}{r}-0.002 \\
(0.003)\end{array}$ & $\begin{array}{r}-0.005 \\
(0.004)\end{array}$ & $\begin{array}{r}-0.008 \\
(0.005)\end{array}$ & $\begin{array}{r}-0.007 \\
(0.009)\end{array}$ & $\begin{array}{r}-0.003 \\
(0.009)\end{array}$ & $\begin{array}{r}-0.003 \\
(0.009)\end{array}$ & $\begin{array}{r}-0.007 \\
(0.009)\end{array}$ \\
\hline $\mathrm{ECM}^{*}$ & tariff levels & & & & $\begin{array}{r}0.015 \\
(0.025)\end{array}$ & $\begin{array}{r}0.011 \\
(0.029)\end{array}$ & $\begin{array}{r}0.002 \\
(0.024)\end{array}$ & $\begin{array}{r}-0.020 \\
(0.021)\end{array}$ & $\begin{array}{r}-0.020 \\
(0.021)\end{array}$ & $\begin{array}{r}-0.001 \\
(0.024)\end{array}$ \\
\hline $\mathrm{ECM}^{*}$ & $\log$ distance & & & & $\begin{array}{l}-0.038^{* * *} \\
(0.012)\end{array}$ & $\begin{array}{l}-0.039 * * * \\
(0.012)\end{array}$ & $\begin{array}{l}-0.048^{* * *} \\
(0.017)\end{array}$ & $\begin{array}{l}-0.052^{* * *} \\
(0.019)\end{array}$ & $\begin{array}{l}-0.052 * * * \\
(0.019)\end{array}$ & $\begin{array}{l}-0.048^{* * *} \\
(0.017)\end{array}$ \\
\hline $\mathrm{ECM}^{*}$ & $\log [$ RGDP(importer)/RGDP(exporter)] & & & & & $\begin{array}{r}0.003 \\
(0.006)\end{array}$ & $\begin{array}{r}0.007 \\
(0.007)\end{array}$ & $\begin{array}{r}0.004 \\
(0.007)\end{array}$ & $\begin{array}{r}0.004 \\
(0.007)\end{array}$ & $\begin{array}{r}0.007 \\
(0.007)\end{array}$ \\
\hline $\mathrm{ECM}^{*}$ & log real wage $(\$)$ & & & & & & $\begin{array}{r}-0.001 \\
(0.015)\end{array}$ & $\begin{array}{r}-0.015 \\
(0.016)\end{array}$ & $\begin{array}{r}-0.015 \\
(0.016)\end{array}$ & $\begin{array}{r}-0.005 \\
(0.016)\end{array}$ \\
\hline $\mathrm{ECM}^{*}$ & long term inflation & & & & & & & $\begin{array}{r}-0.017 \\
(0.052)\end{array}$ & $\begin{array}{r}-0.017 \\
(0.052)\end{array}$ & $\begin{array}{r}-0.030 \\
(0.047)\end{array}$ \\
\hline $\mathrm{ECM}^{*}$ & long term exchange rate variability & & & & & & & $\begin{array}{r}-0.149 \\
(0.107)\end{array}$ & $\begin{array}{r}-0.149 \\
(0.106)\end{array}$ & \\
\hline $\mathrm{ECM}^{*}$ & US Importer dummy & & & & & & & & $\begin{array}{r}0.164 \\
(0.115)\end{array}$ & $\begin{array}{r}0.158 \\
(0.116)\end{array}$ \\
\hline & \# of Observations & 3774 & 3774 & 3774 & 3548 & 3548 & 2625 & 2561 & 2561 & 2625 \\
\hline & Adjusted R-squared & 0.151 & 0.153 & 0.154 & 0.162 & 0.161 & 0.153 & 0.155 & 0.154 & 0.154 \\
\hline & Country dummies & yes & yes & yes & yes & yes & yes & yes & yes & yes \\
\hline & Product dummies & yes & yes & yes & yes & yes & yes & yes & yes & yes \\
\hline
\end{tabular}

Full sample

Product dummies

$*=10 \%, * *=5 \%$, and $* * *=1 \%$ levels of significance

product specific (ps) tariffs 


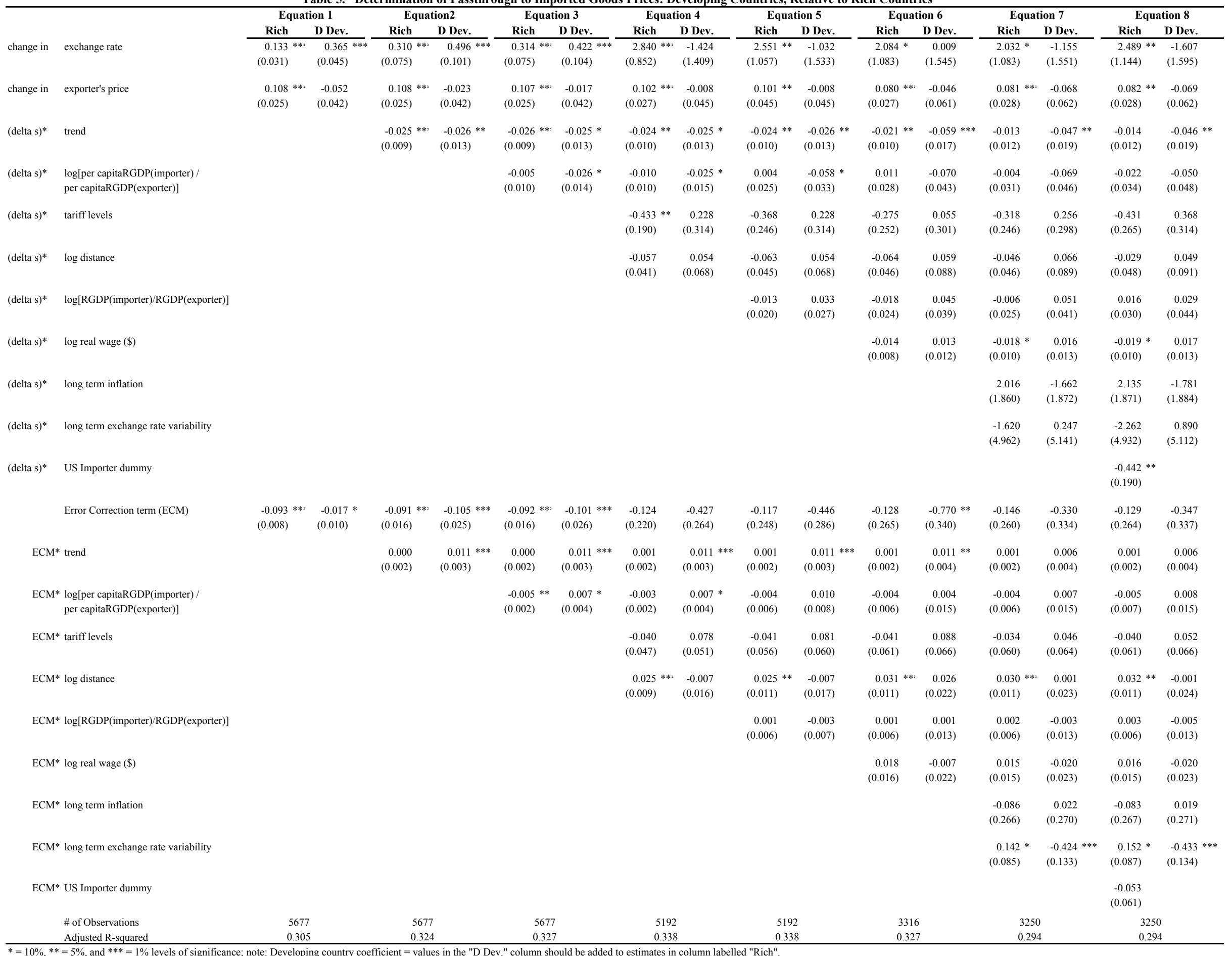


Table 6. Determination of Passthrough to Local Competitor Prices: Developing Countries, Relative to Rich Countries

\begin{tabular}{|c|c|c|c|c|c|c|c|c|c|c|c|c|c|c|c|c|c|}
\hline & & \multicolumn{2}{|c|}{ Equation 1} & \multicolumn{2}{|c|}{ Equation2 } & \multicolumn{2}{|c|}{ Equation 3} & \multicolumn{2}{|c|}{ Equation 4} & \multicolumn{2}{|c|}{ Equation 5} & \multicolumn{2}{|c|}{ Equation 6} & \multicolumn{2}{|c|}{ Equation 7} & \multicolumn{2}{|c|}{ Equation 8} \\
\hline & & Rich & D Dev. & Rich & D Dev. & Rich & D Dev. & Rich & D Dev. & Rich & D Dev. & Rich & D Dev. & Rich & D Dev. & Rich & D Dev. \\
\hline change in & exchange rate & $\begin{array}{r}0.035 \\
(0.031)\end{array}$ & $\begin{array}{l}c^{0.456^{* * *}} \\
(0.044)\end{array}$ & $\begin{array}{l}0.158^{* * *} \\
(0.076)\end{array}$ & $\begin{array}{l}c^{0.676^{* * *}} \\
(0.096)\end{array}$ & $\begin{array}{l}0.168 \text { ** } \\
(0.076)\end{array}$ & $\begin{array}{l}0.656^{* * *} \\
(0.098)\end{array}$ & $\begin{array}{r}-0.439 \\
(0.576)\end{array}$ & $\begin{array}{r}0.773 \\
(0.884)\end{array}$ & $\begin{array}{r}0.839 \\
(0.741)\end{array}$ & $\begin{array}{r}-0.319 \\
(0.999)\end{array}$ & $\begin{aligned} 0.957 \\
(0.772)\end{aligned}$ & $\begin{array}{r}-1.580 \\
(1.301)\end{array}$ & $\begin{array}{r}0.523 \\
(0.874)\end{array}$ & $\begin{array}{r}-1.564 \\
(1.432)\end{array}$ & $\begin{array}{r}-0.129 \\
(0.938)\end{array}$ & $\begin{array}{r}-0.912 \\
(1.470)\end{array}$ \\
\hline change in & exporter's price & $\begin{array}{r}0.046 \\
(0.034)\end{array}$ & $\begin{array}{r}-0.007 \\
(0.051)\end{array}$ & $\begin{array}{r}0.036 \\
(0.034)\end{array}$ & $\begin{array}{r}-0.012 \\
(0.051)\end{array}$ & $\begin{array}{r}0.036 \\
(0.034)\end{array}$ & $\begin{array}{r}-0.011 \\
(0.052)\end{array}$ & $\begin{array}{r}0.020 \\
(0.037)\end{array}$ & $\begin{array}{r}-0.011 \\
(0.056)\end{array}$ & $\begin{array}{r}0.017 \\
(0.045)\end{array}$ & $\begin{array}{r}-0.010 \\
(0.055)\end{array}$ & $\begin{array}{r}0.017 \\
(0.040)\end{array}$ & $\begin{array}{r}-0.020 \\
(0.076)\end{array}$ & $\begin{array}{r}0.014 \\
(0.040)\end{array}$ & $\begin{array}{r}0.000 \\
(0.072)\end{array}$ & $\begin{array}{r}0.013 \\
(0.040)\end{array}$ & $\begin{array}{r}0.000 \\
(0.072)\end{array}$ \\
\hline$\left(\right.$ delta s) ${ }^{*}$ & trend & & & $\begin{array}{l}-0.016 * \\
(0.010)\end{array}$ & $\begin{array}{l}-0.040 * * * \\
(0.012)\end{array}$ & $\begin{array}{l}-0.018 * \\
(0.010)\end{array}$ & $\begin{array}{l}-0.039 * * * \\
(0.012)\end{array}$ & $\begin{array}{l}-0.019 * \\
(0.010)\end{array}$ & $\begin{array}{l}-0.037 \text { *** } \\
(0.013)\end{array}$ & $\begin{array}{l}-0.022 * * \\
(0.010)\end{array}$ & $\begin{array}{l}-0.036 * * * \\
(0.013)\end{array}$ & $\begin{array}{l}-0.022 * * \\
(0.011)\end{array}$ & $\begin{array}{l}-0.073 \# \# \\
(0.016)\end{array}$ & $\begin{array}{r}-0.009 \\
(0.012)\end{array}$ & $\begin{array}{l}-0.054 * * * \\
(0.020)\end{array}$ & $\begin{array}{r}-0.007 \\
(0.012)\end{array}$ & $\begin{array}{l}-0.056 * * * \\
(0.020)\end{array}$ \\
\hline (delta s)* & $\begin{array}{l}\log [\text { per capitaRGDP(importer) / } \\
\text { per capitaRGDP(exporter)] }\end{array}$ & & & & & $\begin{array}{r}-0.014 \\
(0.009)\end{array}$ & $\begin{array}{r}0.009 \\
(0.012)\end{array}$ & $\begin{array}{r}-0.008 \\
(0.009)\end{array}$ & $\begin{array}{r}-0.002 \\
(0.012)\end{array}$ & $\begin{array}{l}-0.062 * *: \\
(0.022)\end{array}$ & $\begin{array}{r}0.024 \\
(0.029)\end{array}$ & $\begin{array}{l}-0.077 * *: \\
(0.026)\end{array}$ & $\begin{array}{c}0.028 \text { \# } \\
(0.047)\end{array}$ & $\begin{array}{l}-0.072 * * \\
(0.030)\end{array}$ & $\begin{array}{r}-0.049 \\
(0.045)\end{array}$ & $\begin{array}{r}-0.045 \\
(0.033)\end{array}$ & $\begin{array}{r}-0.077 \\
(0.047)\end{array}$ \\
\hline$\left(\right.$ delta s)* ${ }^{*}$ & tariff levels & & & & & & & $\begin{array}{r}0.068 \\
(0.123)\end{array}$ & $\begin{array}{r}0.070 \\
(0.196)\end{array}$ & $\begin{array}{r}-0.204 \\
(0.166)\end{array}$ & $\begin{array}{r}0.070 \\
(0.196)\end{array}$ & $\begin{array}{r}-0.244 \\
(0.172)\end{array}$ & $\begin{array}{c}0.223 \# \# \\
(0.226)\end{array}$ & $\begin{array}{r}-0.218 \\
(0.187)\end{array}$ & $\begin{array}{r}0.345 \\
(0.243)\end{array}$ & $\begin{array}{r}-0.056 \\
(0.205)\end{array}$ & $\begin{array}{r}0.183 \\
(0.257)\end{array}$ \\
\hline (delta s)* & log distance & & & & & & & $\begin{array}{r}0.034 \\
(0.042)\end{array}$ & $\begin{array}{r}0.062 \\
(0.065)\end{array}$ & $\begin{array}{r}0.051 \\
(0.044)\end{array}$ & $\begin{array}{r}0.062 \\
(0.065)\end{array}$ & $\begin{array}{r}0.062 \\
(0.046)\end{array}$ & $\begin{array}{r}0.148 \\
(0.092)\end{array}$ & $\begin{array}{r}0.063 \\
(0.050)\end{array}$ & $\begin{array}{r}0.099 \\
(0.090)\end{array}$ & $\begin{array}{r}0.037 \\
(0.052)\end{array}$ & $\begin{array}{r}0.124 \\
(0.091)\end{array}$ \\
\hline$\left(\right.$ delta s)* ${ }^{*}$ & $\log [$ RGDP(importer)/RGDP(exporter)] & & & & & & & & & $\begin{array}{r}0.051 \\
(0.019)\end{array}$ & $\begin{array}{r}-0.022 \\
(0.026)\end{array}$ & $\begin{array}{r}0.066 \\
(0.024)\end{array}$ & $\begin{array}{r}-0.027 \\
(0.043)\end{array}$ & $\begin{array}{l}0.067 \text { **: } \\
(0.025)\end{array}$ & $\begin{array}{r}0.044 \\
(0.042)\end{array}$ & $\begin{array}{r}0.034 \\
(0.029)\end{array}$ & $\begin{array}{l}0.076 * \\
(0.044)\end{array}$ \\
\hline (delta s)* & log real wage $(\$)$ & & & & & & & & & & & $\begin{array}{r}-0.001 \\
(0.008)\end{array}$ & $\begin{array}{r}-0.014 \\
(0.011)\end{array}$ & $\begin{array}{r}-0.006 \\
(0.008)\end{array}$ & $\begin{array}{r}-0.003 \\
(0.011)\end{array}$ & $\begin{array}{r}-0.004 \\
(0.008)\end{array}$ & $\begin{array}{r}-0.004 \\
(0.011)\end{array}$ \\
\hline (delta s)* & long term inflation & & & & & & & & & & & & & $\begin{array}{l}2.980 \text { ** } \\
(1.501)\end{array}$ & $\begin{array}{r}-2.376 \\
(1.527)\end{array}$ & $\begin{array}{l}2.810 * \\
(1.506)\end{array}$ & $\begin{array}{r}-2.206 \\
(1.531)\end{array}$ \\
\hline (delta s)* & long term exchange rate variability & & & & & & & & & & & & & $\begin{array}{r}1.980 \\
(4.541)\end{array}$ & $\begin{array}{r}-4.503 \\
(4.848)\end{array}$ & $\begin{array}{r}2.941 \\
(4.578)\end{array}$ & $\begin{array}{r}-5.463 \\
(4.881)\end{array}$ \\
\hline (delta s)* & US Importer dummy & & & & & & & & & & & & & & & $\begin{array}{l}0.669 \text { *** } \\
(0.215)\end{array}$ & \\
\hline & Error Correction term (ECM) & $\begin{array}{r}-0.006 \\
(0.008)\end{array}$ & $\begin{array}{r}-0.013 \\
(0.010)\end{array}$ & $\begin{array}{c}0.029 * \\
(0.016)\end{array}$ & $\begin{array}{l}-0.032 \\
(0.025)\end{array}$ & $\begin{array}{l}0.028 * \\
(0.016)\end{array}$ & $\begin{array}{r}-0.031 \\
(0.025)\end{array}$ & $\begin{array}{r}-0.046 \\
(0.160)\end{array}$ & $\begin{array}{r}0.105 \\
(0.198)\end{array}$ & $\begin{array}{r}0.136 \\
(0.182)\end{array}$ & $\begin{array}{r}-0.042 \\
(0.217)\end{array}$ & $\begin{array}{r}0.150 \\
(0.189)\end{array}$ & $\begin{array}{l}-0.190 \\
(0.265)\end{array}$ & $\begin{array}{r}0.016 \\
(0.197)\end{array}$ & $\begin{array}{l}-0.181 \\
(0.275)\end{array}$ & $\begin{array}{r}0.015 \\
(0.200)\end{array}$ & $\begin{array}{r}-0.180 \\
(0.276)\end{array}$ \\
\hline $\mathrm{ECM}$ & trend & & & $\begin{array}{l}-0.005 * *: \\
(0.002)\end{array}$ & $\begin{array}{r}0.003 \\
(0.003)\end{array}$ & $\begin{array}{l}-0.004 * * \\
(0.002)\end{array}$ & $\begin{array}{r}0.002 \\
(0.003)\end{array}$ & $\begin{array}{l}-0.005 * * \\
(0.002)\end{array}$ & $\begin{array}{r}0.003 \\
(0.003)\end{array}$ & $\begin{array}{l}-0.005 * *: \\
(0.002)\end{array}$ & $\begin{array}{r}0.003 \\
(0.003)\end{array}$ & $\begin{array}{l}-0.005 * *: \\
(0.002)\end{array}$ & $\begin{array}{l}0.008 * * \\
(0.004)\end{array}$ & $\begin{array}{r}-0.002 \\
(0.002)\end{array}$ & $\begin{array}{r}0.005 \\
(0.005)\end{array}$ & $\begin{array}{r}-0.002 \\
(0.002)\end{array}$ & $\begin{array}{r}0.005 \\
(0.005)\end{array}$ \\
\hline ECM & $\begin{array}{l}\text { log[per capitaRGDP(importer) / } \\
\text { per capitaRGDP(exporter)] }\end{array}$ & & & & & $\begin{array}{l}-0.006 * *: \\
(0.002)\end{array}$ & $\begin{array}{l}0.005 * \\
(0.003)\end{array}$ & $\begin{array}{l}-0.006 * * \\
(0.002)\end{array}$ & $\begin{array}{r}0.004 \\
(0.003)\end{array}$ & $\begin{array}{l}-0.019 * *: \\
(0.006)\end{array}$ & $\begin{array}{r}0.011 \\
(0.007)\end{array}$ & $\begin{array}{l}-0.019 * * \\
(0.007)\end{array}$ & $\begin{array}{r}-0.008 \\
(0.014)\end{array}$ & $\begin{array}{l}-0.019 * *: \\
(0.007)\end{array}$ & $\begin{array}{r}0.000 \\
(0.013)\end{array}$ & $\begin{array}{l}-0.018 * * \\
(0.007)\end{array}$ & $\begin{array}{r}0.000 \\
(0.013)\end{array}$ \\
\hline ECM & " tariff levels & & & & & & & $\begin{array}{r}-0.007 \\
(0.034)\end{array}$ & $\begin{array}{r}0.003 \\
(0.037)\end{array}$ & $\begin{array}{r}-0.052 \\
(0.040)\end{array}$ & $\begin{array}{r}0.045 \\
(0.043)\end{array}$ & $\begin{array}{r}-0.053 \\
(0.043)\end{array}$ & $\begin{array}{r}0.028 \\
(0.050)\end{array}$ & $\begin{array}{r}-0.031 \\
(0.044)\end{array}$ & $\begin{array}{r}0.009 \\
(0.050)\end{array}$ & $\begin{array}{r}-0.030 \\
(0.045)\end{array}$ & $\begin{array}{r}0.008 \\
(0.051)\end{array}$ \\
\hline ECM & " log distance & & & & & & & $\begin{array}{r}0.012 \\
(0.009)\end{array}$ & $\begin{array}{r}-0.018 \\
(0.014)\end{array}$ & $\begin{array}{l}0.020 \text { ** } \\
(0.009)\end{array}$ & $\begin{array}{l}-0.026 * \\
(0.014)\end{array}$ & $\begin{array}{r}0.018 \\
(0.011)\end{array}$ & $\begin{array}{r}0.016 \\
(0.018)\end{array}$ & $\begin{array}{l}0.019 * \\
(0.012)\end{array}$ & $\begin{array}{r}0.019 \\
(0.019)\end{array}$ & $\begin{array}{r}0.018 \\
(0.012)\end{array}$ & $\begin{array}{r}0.020 \\
(0.019)\end{array}$ \\
\hline ECM & $\log [$ RGDP(importer)/RGDP(exporter)] & & & & & & & & & $\begin{array}{l}0.013 \text { **: } \\
(0.005)\end{array}$ & $\begin{array}{l}-0.008 \\
(0.006)\end{array}$ & $\begin{array}{c}0.013 \text { ** } \\
(0.006)\end{array}$ & $\begin{array}{r}0.009 \\
(0.012)\end{array}$ & $\begin{array}{c}0.014 * * \\
(0.006)\end{array}$ & $\begin{array}{l}-0.001 \\
(0.011)\end{array}$ & $\begin{array}{c}0.0133^{* *} \\
(0.006)\end{array}$ & $\begin{array}{r}-0.001 \\
(0.011)\end{array}$ \\
\hline ECM & " log real wage (\$) & & & & & & & & & & & $\begin{array}{r}-0.008 \\
(0.015)\end{array}$ & $\begin{array}{l}0.044{ }^{* *} \\
(0.020)\end{array}$ & $\begin{array}{r}0.002 \\
(0.015)\end{array}$ & $\begin{array}{r}0.018 \\
(0.020)\end{array}$ & $\begin{array}{r}0.002 \\
(0.015)\end{array}$ & $\begin{array}{r}0.018 \\
(0.020)\end{array}$ \\
\hline $\mathrm{ECM}$ & " long term inflation & & & & & & & & & & & & & $\begin{array}{c}0.530 * \\
(0.273)\end{array}$ & $\begin{array}{l}-0.502 * \\
(0.275)\end{array}$ & $\begin{array}{c}0.537 \text { * } \\
(0.274)\end{array}$ & $\begin{array}{l}-0.509 * \\
(0.275)\end{array}$ \\
\hline $\mathrm{ECM}$ & * long term exchange rate variability & & & & & & & & & & & & & $\begin{array}{r}0.099 \\
(0.065)\end{array}$ & $\begin{array}{l}-0.323 * * \\
(0.129)\end{array}$ & $\begin{array}{r}0.085 \\
(0.065)\end{array}$ & $\begin{array}{l}-0.309{ }^{* *} \\
(0.129)\end{array}$ \\
\hline $\mathrm{ECM}$ & " US Importer dummy & & & & & & & & & & & & & & & $\begin{array}{r}-0.003 \\
(0.062)\end{array}$ & \\
\hline & \# of Observations & 5630 & & 5630 & & 5630 & & 5077 & & 5077 & & 3299 & & 3235 & & 3235 & \\
\hline & Adjusted R-squared & 0.236 & & 0.250 & & 0.250 & & 0.255 & & 0.256 & & 0.252 & & 0.264 & & 0.265 & \\
\hline
\end{tabular}


Table 7. Determination of Passthrough to the CPI: Developing Countries, Relative to Rich Countries

\begin{tabular}{|c|c|c|c|c|c|c|c|c|c|c|c|c|c|c|c|}
\hline & & \multicolumn{2}{|c|}{ Equation 1} & \multicolumn{2}{|c|}{ Equation2 } & \multicolumn{2}{|c|}{ Equation 3} & \multicolumn{2}{|c|}{ Equation 4} & \multicolumn{2}{|c|}{ Equation 5} & \multicolumn{2}{|c|}{ Equation 6} & \multicolumn{2}{|c|}{ Equation 7} \\
\hline & & Rich & D Dev. & Rich & D Dev. & Rich & D Dev. & Rich & D Dev. & Rich & D Dev. & Rich & D Dev. & Rich & D Dev. \\
\hline change in & exchange rate & $\begin{array}{r}0.017 \\
(0.011)\end{array}$ & $\begin{array}{l}0.336^{* * *} \\
(0.065)\end{array}$ & $\begin{array}{r}0.001 \\
(0.034)\end{array}$ & $\begin{array}{l}0.677^{* * *} \\
(0.124)\end{array}$ & $\begin{array}{r}-0.022 \\
(0.033)\end{array}$ & $\begin{array}{l}1.131 \text { *** } \\
(0.220)\end{array}$ & $\begin{array}{c}1.620 * \\
(0.851)\end{array}$ & $\begin{array}{r}1.017 \\
(2.038)\end{array}$ & $\begin{array}{c}1.733 * \\
(1.031)\end{array}$ & $\begin{array}{r}2.338 \\
(2.416)\end{array}$ & $\begin{array}{l}1.858 * \\
(1.072)\end{array}$ & $\begin{array}{r}-2.086 \\
(3.377)\end{array}$ & $\begin{array}{c}1.915 * \\
(1.079)\end{array}$ & $\begin{array}{r}-3.681 \\
(2.656)\end{array}$ \\
\hline change in & exporter's price & $\begin{array}{l}-0.021 * *: \\
(0.007)\end{array}$ & $\begin{array}{l}-0.073 \text { *** } \\
(0.028)\end{array}$ & $\begin{array}{l}-0.024 * *: \\
(0.008)\end{array}$ & $\begin{array}{r}0.002 \\
(0.027)\end{array}$ & $\begin{array}{l}-0.023 * *: \\
(0.008)\end{array}$ & $\begin{array}{r}0.013 \\
(0.027)\end{array}$ & $\begin{array}{l}-0.019 * * \\
(0.009)\end{array}$ & $\begin{array}{r}0.022 \\
(0.032)\end{array}$ & $\begin{array}{r}-0.018 \\
(0.045)\end{array}$ & $\begin{array}{r}0.031 \\
(0.033)\end{array}$ & $\begin{array}{r}-0.017 \\
(0.011)\end{array}$ & $\begin{array}{r}0.049 \\
(0.044)\end{array}$ & $\begin{array}{l}-0.019 * \\
(0.011)\end{array}$ & $\begin{array}{r}0.021 \\
(0.031)\end{array}$ \\
\hline (delta s)* & trend & & & $\begin{array}{r}0.002 \\
(0.004)\end{array}$ & $\begin{array}{l}-0.059 * * * \\
(0.013)\end{array}$ & $\begin{array}{r}0.002 \\
(0.004)\end{array}$ & $\begin{array}{l}-0.065 * * * \\
(0.013)\end{array}$ & $\begin{array}{r}0.001 \\
(0.004)\end{array}$ & $\begin{array}{l}-0.063 * * * \\
(0.014)\end{array}$ & $\begin{array}{r}0.001 \\
(0.004)\end{array}$ & $\begin{array}{l}-0.0655^{* * *} \\
(0.014)\end{array}$ & $\begin{array}{r}0.001 \\
(0.004)\end{array}$ & $\begin{array}{l}-0.101 * * * \\
(0.021)\end{array}$ & $\begin{array}{r}0.005 \\
(0.006)\end{array}$ & $\begin{array}{r}0.002 \\
(0.015)\end{array}$ \\
\hline (delta s)* & $\begin{array}{l}\log [\text { per capitaRGDP(importer) / } \\
\text { per capitaRGDP(exporter)] }\end{array}$ & & & & & $\begin{array}{r}-0.063 \\
(0.045)\end{array}$ & $\begin{array}{l}0.2511^{* * *} \\
(0.085)\end{array}$ & $\begin{array}{r}-0.040 \\
(0.046)\end{array}$ & $\begin{array}{l}0.235 \text { ** } \\
(0.098)\end{array}$ & $\begin{array}{r}-0.036 \\
(0.049)\end{array}$ & $\begin{array}{l}0.151 * \\
(0.090)\end{array}$ & $\begin{array}{r}-0.050 \\
(0.088)\end{array}$ & $\begin{array}{l}1.120 * * * \\
(0.248)\end{array}$ & $\begin{array}{r}0.102 \\
(0.102)\end{array}$ & $\begin{array}{l}0.453 \text { *** } \\
(0.167)\end{array}$ \\
\hline (delta s)* & tariff levels & & & & & & & $\begin{array}{l}-0.336 * \\
(0.192)\end{array}$ & $\begin{array}{r}-0.440 \\
(0.493)\end{array}$ & $\begin{array}{r}-0.358 \\
(0.227)\end{array}$ & $\begin{array}{r}-0.440 \\
(0.493)\end{array}$ & $\begin{array}{l}-0.396 * \\
(0.233)\end{array}$ & $\begin{array}{r}0.153 \\
(0.638)\end{array}$ & $\begin{array}{l}-0.452 * * \\
(0.220)\end{array}$ & $\begin{array}{l}1.072 \text { ** } \\
(0.528)\end{array}$ \\
\hline (delta s) ${ }^{*}$ & log distance & & & & & & & $\begin{array}{r}-0.007 \\
(0.024)\end{array}$ & $\begin{array}{r}0.122 \\
(0.109)\end{array}$ & $\begin{array}{r}-0.006 \\
(0.024)\end{array}$ & $\begin{array}{r}0.122 \\
(0.109)\end{array}$ & $\begin{array}{r}0.000 \\
(0.027)\end{array}$ & $\begin{array}{r}0.086 \\
(0.290)\end{array}$ & $\begin{array}{r}0.009 \\
(0.025)\end{array}$ & $\begin{array}{r}-0.194 \\
(0.165)\end{array}$ \\
\hline (delta s)* & $\log [$ RGDP(importer)/RGDP(exporter)] & & & & & & & & & $\begin{array}{r}0.003 \\
(0.009)\end{array}$ & $\begin{array}{l}0.079 * \\
(0.041)\end{array}$ & $\begin{array}{r}0.007 \\
(0.010)\end{array}$ & $\begin{array}{l}-0.099 * \\
(0.056)\end{array}$ & $\begin{array}{r}0.003 \\
(0.012)\end{array}$ & $\begin{array}{r}0.008 \\
(0.048)\end{array}$ \\
\hline (delta s)* & log real wage (\$) & & & & & & & & & & & $\begin{array}{r}-0.002 \\
(0.039)\end{array}$ & $\begin{array}{l}-0.684 * * * \\
(0.094)\end{array}$ & $\begin{array}{r}-0.020 \\
(0.042)\end{array}$ & $\begin{array}{l}-0.369 * * * \\
(0.071)\end{array}$ \\
\hline (delta s)* & long term inflation & & & & & & & & & & & & & $\begin{array}{r}0.920 \\
(0.938)\end{array}$ & $\begin{array}{r}0.055 \\
(0.954)\end{array}$ \\
\hline$\left(\right.$ delta s) ${ }^{*}$ & long term exchange rate variability & & & & & & & & & & & & & $\begin{array}{l}2.301 * * \\
(1.133)\end{array}$ & $\begin{array}{l}-7.133 * * * \\
(1.549)\end{array}$ \\
\hline & Error Correction term (ECM) & $\begin{array}{l}-0.037 * *: \\
(0.012)\end{array}$ & $\begin{array}{c}0.025 * \\
(0.014)\end{array}$ & $\begin{array}{l}-0.037 \text { **: } \\
(0.012)\end{array}$ & $\begin{array}{l}0.036 * * * \\
(0.014)\end{array}$ & $\begin{array}{l}-0.039 * *: \\
(0.013)\end{array}$ & $\begin{array}{l}0.039 \text { *** } \\
(0.014)\end{array}$ & $\begin{array}{r}-0.989 \\
(0.704)\end{array}$ & $\begin{array}{r}0.697 \\
(0.753)\end{array}$ & $\begin{array}{r}-0.923 \\
(0.700)\end{array}$ & $\begin{array}{r}0.617 \\
(0.749)\end{array}$ & $\begin{array}{r}-0.984 \\
(0.719)\end{array}$ & $\begin{array}{r}0.216 \\
(0.897)\end{array}$ & $\begin{array}{r}-0.807 \\
(0.684)\end{array}$ & $\begin{array}{r}1.155 \\
(0.706)\end{array}$ \\
\hline $\mathrm{ECM}$ & * trend & & & $\begin{array}{r}0.000 \\
(0.000)\end{array}$ & $\begin{array}{l}0.003 * * * \\
(0.001)\end{array}$ & $\begin{array}{r}0.000 \\
(0.001)\end{array}$ & $\begin{array}{l}0.006 \text { *** } \\
(0.002)\end{array}$ & $\begin{array}{r}0.000 \\
(0.001)\end{array}$ & $\begin{array}{l}0.008 \text { *** } \\
(0.002)\end{array}$ & $\begin{array}{r}0.000 \\
(0.001)\end{array}$ & $\begin{array}{l}0.008 * * * \\
(0.002)\end{array}$ & $\begin{array}{r}-0.002 \\
(0.001)\end{array}$ & $\begin{array}{l}0.015 * * \\
(0.006)\end{array}$ & $\begin{array}{r}0.001 \\
(0.002)\end{array}$ & $\begin{array}{l}0.010 * * * \\
(0.004)\end{array}$ \\
\hline ECM & $\begin{array}{l}\text { * log[per capitaRGDP(importer) / } \\
\text { per capitaRGDP(exporter)] }\end{array}$ & & & & & $\begin{array}{r}-0.008 \\
(0.016)\end{array}$ & $\begin{array}{r}0.032 \\
(0.021)\end{array}$ & $\begin{array}{r}-0.018 \\
(0.015)\end{array}$ & $\begin{array}{l}0.057 \text { *** } \\
(0.021)\end{array}$ & $\begin{array}{r}-0.012 \\
(0.017)\end{array}$ & $\begin{array}{l}0.046 * * \\
(0.022)\end{array}$ & $\begin{array}{r}0.023 \\
(0.041)\end{array}$ & $\begin{array}{r}0.022 \\
(0.108)\end{array}$ & $\begin{array}{r}0.022 \\
(0.039)\end{array}$ & $\begin{array}{r}-0.117 \\
(0.088)\end{array}$ \\
\hline ECM & * tariff levels & & & & & & & $\begin{array}{r}0.207 \\
(0.149)\end{array}$ & $\begin{array}{r}-0.144 \\
(0.159)\end{array}$ & $\begin{array}{r}0.193 \\
(0.148)\end{array}$ & $\begin{array}{r}-0.127 \\
(0.158)\end{array}$ & $\begin{array}{r}0.205 \\
(0.152)\end{array}$ & $\begin{array}{r}-0.038 \\
(0.190)\end{array}$ & $\begin{array}{r}0.166 \\
(0.144)\end{array}$ & $\begin{array}{r}-0.241 \\
(0.149)\end{array}$ \\
\hline ECM & * $\log$ distance & & & & & & & $\begin{array}{r}-0.001 \\
(0.001)\end{array}$ & $\begin{array}{r}0.001 \\
(0.004)\end{array}$ & $\begin{array}{r}0.001 \\
(0.002)\end{array}$ & $\begin{array}{r}0.001 \\
(0.006)\end{array}$ & $\begin{array}{r}0.001 \\
(0.003)\end{array}$ & $\begin{array}{r}-0.009 \\
(0.013)\end{array}$ & $\begin{array}{r}-0.001 \\
(0.004)\end{array}$ & $\begin{array}{r}0.006 \\
(0.009)\end{array}$ \\
\hline ECM & * $\log [$ RGDP(importer)/RGDP(exporter)] & & & & & & & & & $\begin{array}{l}0.005 * \\
(0.003)\end{array}$ & $\begin{array}{r}0.002 \\
(0.008)\end{array}$ & $\begin{array}{l}0.010 * * \\
(0.003)\end{array}$ & $\begin{array}{r}-0.004 \\
(0.015)\end{array}$ & $\begin{array}{l}0.008 \text { ** } \\
(0.003)\end{array}$ & $\begin{array}{r}-0.002 \\
(0.012)\end{array}$ \\
\hline $\mathrm{ECM}$ & * log real wage $(\$)$ & & & & & & & & & & & $\begin{array}{r}-0.019 \\
(0.016)\end{array}$ & $\begin{array}{r}0.016 \\
(0.052)\end{array}$ & $\begin{array}{r}-0.012 \\
(0.015)\end{array}$ & $\begin{array}{l}0.085 * \\
(0.044)\end{array}$ \\
\hline ECM & * long term inflation & & & & & & & & & & & & & $\begin{array}{r}0.473 \\
(0.485)\end{array}$ & $\begin{array}{r}-0.629 \\
(0.487)\end{array}$ \\
\hline $\mathrm{ECM}$ & * long term exchange rate variability & & & & & & & & & & & & & $\begin{array}{r}-0.007 \\
(0.034)\end{array}$ & $\begin{array}{r}0.156 \\
(0.097)\end{array}$ \\
\hline & $\begin{array}{l}\text { \# of Observations } \\
\text { Adjusted R-squared }\end{array}$ & $\begin{array}{c}732 \\
0.716\end{array}$ & & $\begin{array}{l}710 \\
0.747\end{array}$ & & $\begin{array}{l}710 \\
0.767\end{array}$ & & $\begin{array}{c}579 \\
0.778\end{array}$ & & $\begin{array}{r}579 \\
0.786\end{array}$ & & $\begin{array}{c}350 \\
0.861\end{array}$ & & $\begin{array}{c}345 \\
0.907\end{array}$ & \\
\hline
\end{tabular}


Table 8. Determination of Passthrough to Unit Values: Developing Countries, Relative to Rich Countries

\begin{tabular}{|c|c|c|c|c|c|c|c|c|c|c|c|c|c|c|c|c|c|}
\hline \multirow{3}{*}{ change in } & \multirow[b]{3}{*}{ exchange rate } & \multicolumn{2}{|c|}{ Equation 1} & \multicolumn{2}{|c|}{ Equation2 } & \multicolumn{2}{|c|}{ Equation 3} & \multicolumn{2}{|c|}{ Equation 4} & \multicolumn{2}{|c|}{ Equation 5} & \multicolumn{2}{|c|}{ Equation 6} & \multicolumn{2}{|c|}{ Equation 7} & \multicolumn{2}{|c|}{ Equation 8} \\
\hline & & Rich & D Dev. & Rich & D Dev. & Rich & D Dev. & Rich & D Dev. & Rich & D Dev. & Rich & D Dev. & Rich & D Dev. & Rich & D Dev. \\
\hline & & $\begin{array}{l}0.681 \text { **: } \\
(0.112)\end{array}$ & $\begin{array}{l}-0.022 \\
(0.144)\end{array}$ & $\begin{array}{l}0.516 * * \\
(0.262)\end{array}$ & $\begin{aligned}-0.139 \\
(0.317)\end{aligned}$ & $\begin{array}{c}0.485 * \\
(0.265)\end{array}$ & $\begin{array}{l}-0.062 \\
(0.321)\end{array}$ & $\begin{array}{l}8.496 \text { **: } \\
(1.781)\end{array}$ & $\begin{array}{l}-3.408 \\
(2.355)\end{array}$ & $\begin{array}{l}8.144 \text { **: } \\
(2.557)\end{array}$ & $\begin{array}{l}-3.142 \\
(3.024)\end{array}$ & $\begin{array}{l}7.244 * *: \\
(2.404)\end{array}$ & $\begin{array}{l}-7.188^{* *} \\
(3.012)\end{array}$ & $\begin{array}{l}6.966 * *: \\
(2.459)\end{array}$ & $\begin{array}{l}-5.754 * \\
(3.114)\end{array}$ & $\begin{array}{l}7.422 * * \\
(2.647)\end{array}$ & $\begin{array}{l}-6.213 * \\
(3.260)\end{array}$ \\
\hline change in & exporter's price & $\begin{array}{l}0.226 * *: \\
(0.069)\end{array}$ & $\begin{array}{l}-0.237 * * \\
(0.116)\end{array}$ & $\begin{array}{l}0.322 \text { **: } \\
(0.077)\end{array}$ & $\begin{array}{l}-0.328 * * * \\
(0.121)\end{array}$ & $\begin{array}{l}0.322 * *: \\
(0.077)\end{array}$ & $\begin{array}{l}-0.327 * * * \\
(0.121)\end{array}$ & $\begin{array}{l}0.327 \text { **: } \\
(0.081)\end{array}$ & $\begin{array}{l}-0.336 * * * \\
(0.126)\end{array}$ & $\begin{array}{l}0.326^{* *} \\
(0.045)\end{array}$ & $\begin{array}{l}-0.333 * * * \\
(0.126)\end{array}$ & $\begin{array}{l}0.307 * *: \\
(0.084)\end{array}$ & $\begin{array}{r}-0.082 \\
(0.144)\end{array}$ & $\begin{array}{l}0.3111^{* *}: \\
(0.084)\end{array}$ & $\begin{array}{r}-0.104 \\
(0.152)\end{array}$ & $\begin{array}{l}0.309 * * \\
(0.084)\end{array}$ & $\begin{array}{r}-0.102 \\
(0.151)\end{array}$ \\
\hline$($ delta s)* & trend & & & $\begin{array}{r}0.029 \\
(0.028)\end{array}$ & $\begin{array}{r}0.011 \\
(0.035)\end{array}$ & $\begin{array}{r}0.033 \\
(0.028)\end{array}$ & $\begin{array}{r}0.009 \\
(0.035)\end{array}$ & $\begin{array}{r}0.016 \\
(0.032)\end{array}$ & $\begin{array}{r}0.032 \\
(0.039)\end{array}$ & $\begin{array}{r}0.017 \\
(0.035)\end{array}$ & $\begin{array}{r}0.032 \\
(0.041)\end{array}$ & $\begin{array}{r}0.024 \\
(0.033)\end{array}$ & $\begin{array}{c}0.042 \# \text { \# } \\
(0.045)\end{array}$ & $\begin{array}{r}-0.039 \\
(0.055)\end{array}$ & $\begin{array}{r}0.082 \\
(0.066)\end{array}$ & $\begin{array}{c}-0.039 \\
(0.055)\end{array}$ & $\begin{array}{r}0.082 \\
(0.066)\end{array}$ \\
\hline$\left(\right.$ delta s)* ${ }^{*}$ & $\begin{array}{l}\log [\text { per capitaRGDP(importer) / } \\
\text { per capitaRGDP(exporter) }]\end{array}$ & & & & & $\begin{array}{r}0.032 \\
(0.020)\end{array}$ & $\begin{array}{r}-0.003 \\
(0.027)\end{array}$ & $\begin{array}{r}0.010 \\
(0.020)\end{array}$ & $\begin{array}{r}0.028 \\
(0.027)\end{array}$ & $\begin{array}{r}0.018 \\
(0.085)\end{array}$ & $\begin{array}{r}0.028 \\
(0.097)\end{array}$ & $\begin{array}{r}0.031 \\
(0.088)\end{array}$ & $\begin{array}{c}0.142 \# \\
(0.115)\end{array}$ & $\begin{array}{l}0.127 * \\
(0.067)\end{array}$ & $\begin{array}{r}0.037 \\
(0.112)\end{array}$ & $\begin{array}{r}0.108 \\
(0.077)\end{array}$ & $\begin{array}{r}0.055 \\
(0.118)\end{array}$ \\
\hline$($ delta s)* & tariff levels & & & & & & & $\begin{array}{l}-1.446 \text { **: } \\
(0.420)\end{array}$ & $\begin{array}{r}0.988 \\
(0.699)\end{array}$ & $\begin{array}{l}-1.358 \text { ** } \\
(0.632)\end{array}$ & $\begin{array}{r}0.988 \\
(0.699)\end{array}$ & $\begin{array}{l}-1.248 \text { ** } \\
(0.598)\end{array}$ & $\begin{array}{c}1.442 \text { \#\# } \\
(0.677)\end{array}$ & $\begin{array}{l}-1.224 * * \\
(0.611)\end{array}$ & $\begin{array}{l}1.401 * * \\
(0.687)\end{array}$ & $\begin{array}{l}-1.337 \text { ** } \\
(0.678)\end{array}$ & $\begin{array}{l}1.514 * * \\
(0.746)\end{array}$ \\
\hline$\left(\right.$ delta s) ${ }^{*}$ & $\log$ distance & & & & & & & $\begin{array}{r}-0.111 \\
(0.141)\end{array}$ & $\begin{array}{r}-0.196 \\
(0.185)\end{array}$ & $\begin{array}{r}-0.122 \\
(0.156)\end{array}$ & $\begin{array}{r}-0.196 \\
(0.185)\end{array}$ & $\begin{array}{l}-0.085 \text { **: } \\
(0.157)\end{array}$ & $\begin{array}{r}-0.032 \\
(0.192)\end{array}$ & $\begin{array}{r}0.003 \\
(0.237)\end{array}$ & $\begin{array}{r}-0.211 \\
(0.263)\end{array}$ & $\begin{array}{r}0.021 \\
(0.248)\end{array}$ & $\begin{array}{r}-0.228 \\
(0.272)\end{array}$ \\
\hline$($ delta s)* & $\log [$ RGDP(importer)/RGDP(exporter)] & & & & & & & & & $\begin{array}{r}-0.010 \\
(0.081)\end{array}$ & $\begin{array}{r}0.001 \\
(0.093)\end{array}$ & $\begin{array}{r}-0.017 \\
(0.085)\end{array}$ & $\begin{array}{r}-0.174 \\
(0.109)\end{array}$ & $\begin{array}{l}-0.133 \text { ** } \\
(0.056)\end{array}$ & $\begin{array}{r}-0.063 \\
(0.099)\end{array}$ & $\begin{array}{r}-0.109 \\
(0.070)\end{array}$ & $\begin{array}{r}-0.086 \\
(0.107)\end{array}$ \\
\hline$\left(\right.$ delta s) ${ }^{*}$ & log real wage (\$) & & & & & & & & & & & $\begin{array}{r}0.003 \\
(0.023)\end{array}$ & $\begin{array}{r}-0.007 \\
(0.030)\end{array}$ & $\begin{array}{r}-0.055 \\
(0.050)\end{array}$ & $\begin{array}{r}0.059 \\
(0.055)\end{array}$ & $\begin{array}{r}-0.056 \\
(0.050)\end{array}$ & $\begin{array}{r}0.059 \\
(0.055)\end{array}$ \\
\hline$($ delta s)* & long term inflation & & & & & & & & & & & & & $\begin{array}{l}-16.703 * * \\
(8.483)\end{array}$ & $\begin{array}{l}15.998 * \\
(8.487)\end{array}$ & $\begin{array}{l}-16.205 * \\
(8.284)\end{array}$ & $\begin{array}{l}15.500 * \\
(8.288)\end{array}$ \\
\hline$($ delta s)* & long term exchange rate variability & & & & & & & & & & & & & $\begin{array}{r}8.604 \\
(7.164)\end{array}$ & $\begin{array}{r}-6.531 \\
(7.504)\end{array}$ & $\begin{array}{r}7.571 \\
(7.342)\end{array}$ & $\begin{array}{r}-5.498 \\
(7.679)\end{array}$ \\
\hline$($ delta s)* & US Importer dummy & & & & & & & & & & & & & & & $\begin{array}{r}-0.572 \\
(0.482)\end{array}$ & \\
\hline & Error Correction term (ECM) & $\begin{array}{l}-0.210 * *: \\
(0.036)\end{array}$ & $\begin{array}{r}-0.014 \\
(0.016)\end{array}$ & $\begin{array}{l}-0.145 * *: \\
(0.034)\end{array}$ & $\begin{array}{l}-0.082 * * \\
(0.035)\end{array}$ & $\begin{array}{l}-0.146 * *: \\
(0.035)\end{array}$ & $\begin{array}{l}-0.094 * * \\
(0.038)\end{array}$ & $\begin{array}{r}0.094 \\
(0.243)\end{array}$ & $\begin{array}{r}-0.116 \\
(0.292)\end{array}$ & $\begin{array}{r}0.090 \\
(0.251)\end{array}$ & $\begin{array}{r}-0.201 \\
(0.306)\end{array}$ & $\begin{array}{r}0.190 \\
(0.195)\end{array}$ & $\begin{array}{r}0.234 \\
(0.268)\end{array}$ & $\begin{array}{r}0.171 \\
(0.214)\end{array}$ & $\begin{array}{r}0.429 \\
(0.283)\end{array}$ & $\begin{array}{r}0.169 \\
(0.214)\end{array}$ & $\begin{array}{r}0.432 \\
(0.284)\end{array}$ \\
\hline $\mathrm{ECM}^{*}$ & trend & & & $\begin{array}{l}-0.010 * *: \\
(0.003)\end{array}$ & $\begin{array}{l}0.010 \text { ** } \\
(0.004)\end{array}$ & $\begin{array}{l}-0.010^{* *:} \\
(0.003)\end{array}$ & $\begin{array}{l}0.010 * * \\
(0.004)\end{array}$ & $\begin{array}{l}-0.011 \text { **: } \\
(0.003)\end{array}$ & $\begin{array}{l}0.010 \text { ** } \\
(0.005)\end{array}$ & $\begin{array}{l}-0.010 * *: \\
(0.003)\end{array}$ & $\begin{array}{l}0.010 \text { ** } \\
(0.005)\end{array}$ & $\begin{array}{l}-0.010 * *: \\
(0.003)\end{array}$ & $\begin{array}{l}0.012 * * \\
(0.006)\end{array}$ & $\begin{array}{l}-0.007 * \\
(0.004)\end{array}$ & $\begin{array}{r}0.006 \\
(0.006)\end{array}$ & $\begin{array}{l}-0.007 * \\
(0.004)\end{array}$ & $\begin{array}{r}0.006 \\
(0.006)\end{array}$ \\
\hline $\mathrm{ECM}^{*}$ & $\begin{array}{l}\log [\text { per capitaRGDP(importer) / } \\
\text { per capitaRGDP(exporter)] }\end{array}$ & & & & & $\begin{array}{r}-0.002 \\
(0.003)\end{array}$ & $\begin{array}{r}-0.002 \\
(0.004)\end{array}$ & $\begin{array}{r}-0.005 \\
(0.004)\end{array}$ & $\begin{array}{r}-0.001 \\
(0.004)\end{array}$ & $\begin{array}{l}-0.016 * \\
(0.009)\end{array}$ & $\begin{array}{r}0.015 \\
(0.012)\end{array}$ & $\begin{array}{r}-0.010 \\
(0.011)\end{array}$ & $\begin{array}{r}0.000 \\
(0.020)\end{array}$ & $\begin{array}{r}-0.009 \\
(0.010)\end{array}$ & $\begin{array}{r}0.008 \\
(0.018)\end{array}$ & $\begin{array}{r}-0.009 \\
(0.010)\end{array}$ & $\begin{array}{r}0.008 \\
(0.018)\end{array}$ \\
\hline $\mathrm{ECM}^{*}$ & tariff levels & & & & & & & $\begin{array}{r}0.022 \\
(0.081)\end{array}$ & $\begin{array}{r}-0.001 \\
(0.084)\end{array}$ & $\begin{array}{r}0.018 \\
(0.085)\end{array}$ & $\begin{array}{r}0.013 \\
(0.088)\end{array}$ & $\begin{array}{r}0.020 \\
(0.064)\end{array}$ & $\begin{array}{r}-0.035 \\
(0.070)\end{array}$ & $\begin{array}{r}0.012 \\
(0.057)\end{array}$ & $\begin{array}{r}-0.056 \\
(0.063)\end{array}$ & $\begin{array}{r}0.012 \\
(0.058)\end{array}$ & $\begin{array}{r}-0.056 \\
(0.064)\end{array}$ \\
\hline $\mathrm{ECM}^{*}$ & $\log$ distance & & & & & & & $\begin{array}{l}-0.041 * \\
(0.025)\end{array}$ & $\begin{array}{r}0.005 \\
(0.027)\end{array}$ & $\begin{array}{r}-0.037 \\
(0.028)\end{array}$ & $\begin{array}{r}0.003 \\
(0.030)\end{array}$ & $\begin{array}{r}-0.050 \\
(0.031)\end{array}$ & $\begin{array}{r}0.000 \\
(0.032)\end{array}$ & $\begin{array}{c}-0.047 \text { * } \\
(0.028)\end{array}$ & $\begin{array}{r}-0.011 \\
(0.028)\end{array}$ & $\begin{array}{c}-0.047 * \\
(0.028)\end{array}$ & $\begin{array}{c}-0.011 \\
(0.028)\end{array}$ \\
\hline $\mathrm{ECM}^{*}$ & $\log [$ RGDP(importer)/RGDP(exporter)] & & & & & & & & & $\begin{array}{r}0.011 \\
(0.009)\end{array}$ & $\begin{array}{r}-0.017 \\
(0.012)\end{array}$ & $\begin{array}{r}0.009 \\
(0.009)\end{array}$ & $\begin{array}{r}0.004 \\
(0.017)\end{array}$ & $\begin{array}{r}0.009 \\
(0.008)\end{array}$ & $\begin{array}{r}-0.003 \\
(0.015)\end{array}$ & $\begin{array}{r}0.008 \\
(0.008)\end{array}$ & $\begin{array}{r}-0.003 \\
(0.015)\end{array}$ \\
\hline $\mathrm{ECM}^{*}$ & log real wage (\$) & & & & & & & & & & & $\begin{array}{r}-0.023 \\
(0.026)\end{array}$ & $\begin{array}{r}0.046 \\
(0.031)\end{array}$ & $\begin{array}{r}-0.020 \\
(0.022)\end{array}$ & $\begin{array}{r}0.027 \\
(0.027)\end{array}$ & $\begin{array}{c}-0.020 \\
(0.022)\end{array}$ & $\begin{array}{r}0.027 \\
(0.027)\end{array}$ \\
\hline $\mathrm{ECM}^{+}$ & long term inflation & & & & & & & & & & & & & $\begin{array}{r}0.512 \\
(0.532)\end{array}$ & $\begin{array}{c}-0.542 \\
(0.537)\end{array}$ & $\begin{array}{r}0.508 \\
(0.531)\end{array}$ & $\begin{array}{c}-0.538 \\
(0.535)\end{array}$ \\
\hline $\mathrm{ECM}^{*}$ & long term exchange rate variability & & & & & & & & & & & & & $\begin{array}{c}-0.429 * \\
(0.260)\end{array}$ & $\begin{array}{c}0.491 \text { * } \\
(0.278)\end{array}$ & $\begin{array}{l}-0.428 * \\
(0.259)\end{array}$ & $\begin{array}{c}0.490 * \\
(0.277)\end{array}$ \\
\hline $\mathrm{ECM}^{*}$ & US Importer dummy & & & & & & & & & & & & & & & $\begin{array}{r}0.136 \\
(0.104)\end{array}$ & \\
\hline & $\begin{array}{l}\text { \# of Observations } \\
\text { Adiusted R-squared }\end{array}$ & $\begin{array}{l}3774 \\
0.152\end{array}$ & & $\begin{array}{l}3774 \\
0.155\end{array}$ & & $\begin{array}{l}3774 \\
0.156\end{array}$ & & $\begin{array}{l}3548 \\
0.163\end{array}$ & & $\begin{array}{l}3548 \\
0.163\end{array}$ & & $\begin{array}{l}2625 \\
0.158\end{array}$ & & $\begin{array}{l}2561 \\
0.165\end{array}$ & & $\begin{array}{l}2561 \\
0.165\end{array}$ & \\
\hline
\end{tabular}


Appendix Table 1: Pass-through to Imported Good Prices: Restricted Sample

\begin{tabular}{|c|c|c|c|c|c|c|c|c|c|c|}
\hline change in & exchange rate & $\begin{array}{c}\text { Eqn 1 } \\
0.5655^{* * *} \\
(0.029)\end{array}$ & $\begin{array}{c}\text { Eqn2 } \\
0.796 \text { *** } \\
(0.064)\end{array}$ & $\begin{array}{c}\text { Eqn3 } \\
0.703 \text { *** } \\
(0.065)\end{array}$ & $\begin{array}{c}\text { Eqn 4 } \\
4.519 * * * \\
(0.597)\end{array}$ & $\begin{array}{c}\text { Eqn 5 } \\
4.807 \text { *** } \\
(0.639)\end{array}$ & $\begin{array}{c}\text { Eqn 6 } \\
4.327 \text { *** } \\
(1.002)\end{array}$ & $\begin{array}{c}\text { Eqn } 7 \\
2.186 * * \\
(0.924)\end{array}$ & $\begin{array}{c}\text { Eqn 8 } \\
2.282 * * \\
(0.941)\end{array}$ & $\begin{array}{c}\text { Eqn 9 } \\
3.762 * * * \\
(0.991)\end{array}$ \\
\hline change in & exporter's price & $\begin{array}{l}0.053 * \\
(0.031)\end{array}$ & $\begin{array}{l}0.073 * * \\
(0.031)\end{array}$ & $\begin{array}{l}0.084^{* * *} \\
(0.030)\end{array}$ & $\begin{array}{l}0.083^{* * *} \\
(0.032)\end{array}$ & $\begin{array}{l}0.083 * * * \\
(0.032)\end{array}$ & $\begin{array}{l}0.081 * * \\
(0.037)\end{array}$ & $\begin{array}{l}0.077^{* *} \\
(0.036)\end{array}$ & $\begin{array}{l}0.078 * * \\
(0.036)\end{array}$ & $\begin{array}{l}0.080^{* *} \\
(0.037)\end{array}$ \\
\hline$($ delta s)* & trend & & $\begin{array}{l}-0.036^{* * *} \\
(0.009)\end{array}$ & $\begin{array}{l}-0.042 * * * \\
(0.009)\end{array}$ & $\begin{array}{l}-0.044^{* * *} \\
(0.009)\end{array}$ & $\begin{array}{l}-0.044^{* * *} \\
(0.009)\end{array}$ & $\begin{array}{l}-0.052 * * * \\
(0.011)\end{array}$ & $\begin{array}{l}-0.041^{* * *} \\
(0.011)^{2}\end{array}$ & $\begin{array}{l}-0.041^{* * *} \\
(0.011)\end{array}$ & $\begin{array}{l}-0.027^{* *} \\
(0.011)\end{array}$ \\
\hline$(\text { delta } \mathrm{s})^{*}$ & $\begin{array}{l}\log [\text { per capitaRGDP(importer) / } \\
\text { per capitaRGDP(exporter)] }\end{array}$ & & & $\begin{array}{l}-0.051^{* * *} \\
(0.009)^{*}\end{array}$ & $\begin{array}{l}-0.051 * * * \\
(0.010)\end{array}$ & $\begin{array}{l}-0.076^{* * *} \\
(0.017)\end{array}$ & $\begin{array}{l}-0.100 * * * \\
(0.027)\end{array}$ & $\begin{array}{r}-0.030 \\
(0.025)\end{array}$ & $\begin{array}{r}-0.034 \\
(0.026)\end{array}$ & $\begin{array}{l}-0.085 * * * \\
(0.028)\end{array}$ \\
\hline$($ delta s)* & tariff levels & & & & $\begin{array}{l}-0.535^{* * *} \\
(0.101)\end{array}$ & $\begin{array}{l}-0.602 * * * \\
(0.111)^{* *}\end{array}$ & $\begin{array}{l}-0.405 * * \\
(0.165)\end{array}$ & $\begin{array}{r}-0.085 \\
(0.162)\end{array}$ & $\begin{array}{r}-0.101 \\
(0.166)\end{array}$ & $\begin{array}{l}-0.345 \text { ** } \\
(0.162)\end{array}$ \\
\hline$($ delta s)* & $\log$ distance & & & & $\begin{array}{l}-0.1388^{* * *} \\
(0.042)\end{array}$ & $\begin{array}{l}-0.129^{* * *} \\
(0.042)\end{array}$ & $\begin{array}{l}-0.180 \text { *** } \\
(0.056)\end{array}$ & $\begin{array}{l}-0.144^{* * *} \\
(0.054)\end{array}$ & $\begin{array}{l}-0.145 * * * \\
(0.054)\end{array}$ & $\begin{array}{l}-0.182 \text { *** } \\
(0.056)\end{array}$ \\
\hline$($ delta s)* & $\log [$ RGDP(importer)/RGDP(exporter)] & & & & & $\begin{array}{c}0.027 \text { * } \\
(0.016)\end{array}$ & $\begin{array}{l}0.059 * * \\
(0.025)\end{array}$ & $\begin{array}{r}-0.006 \\
(0.021)\end{array}$ & $\begin{array}{r}-0.002 \\
(0.022)\end{array}$ & $\begin{array}{l}0.052 \text { ** } \\
(0.026)\end{array}$ \\
\hline$($ delta s)* & log real wage $(\$)$ & & & & & & $\begin{array}{l}-0.020 * * \\
(0.009)\end{array}$ & $\begin{array}{r}-0.011 \\
(0.009)\end{array}$ & $\begin{array}{r}-0.011 \\
(0.009)\end{array}$ & $\begin{array}{l}-0.016 * \\
(0.008)\end{array}$ \\
\hline$($ delta s)* & long term inflation & & & & & & & $\begin{array}{r}0.174 \\
(0.185)\end{array}$ & $\begin{array}{r}0.175 \\
(0.185)\end{array}$ & $\begin{array}{l}0.259 \text { *** } \\
(0.063)\end{array}$ \\
\hline$($ delta s)* & long term exchange rate variability & & & & & & & $\begin{array}{r}0.075 \\
(1.151)\end{array}$ & $\begin{array}{r}0.060 \\
(1.152)\end{array}$ & \\
\hline$(\text { delta } s)^{*}$ & US Importer dummy & & & & & & & & $\begin{array}{r}-0.263 \\
(0.164)\end{array}$ & $\begin{array}{l}-0.509 \text { *** } \\
(0.172)\end{array}$ \\
\hline & Error Correction term (ECM) & $\begin{array}{l}-0.086 \text { *** } \\
(0.007)\end{array}$ & $\begin{array}{l}-0.148^{* * *} \\
(0.017)\end{array}$ & $\begin{array}{l}-0.146 \text { *** } \\
(0.016)\end{array}$ & $\begin{array}{l}-0.259 * * \\
(0.106)\end{array}$ & $\begin{array}{l}-0.223 * \\
(0.117)\end{array}$ & $\begin{array}{l}-0.265 * \\
(0.158)\end{array}$ & $\begin{array}{l}-0.278 * \\
(0.154)\end{array}$ & $\begin{array}{l}-0.265 * \\
(0.156)\end{array}$ & $\begin{array}{r}-0.195 \\
(0.162)\end{array}$ \\
\hline $\mathrm{ECM}^{*}$ & trend & & $\begin{array}{l}0.008^{* * *} \\
(0.002)\end{array}$ & $\begin{array}{l}0.008^{* * *} \\
(0.002)\end{array}$ & $\begin{array}{l}0.008^{* * *} \\
(0.002)\end{array}$ & $\begin{array}{l}0.008^{* * *} \\
(0.002)\end{array}$ & $\begin{array}{l}0.008 \text { *** } \\
(0.002)\end{array}$ & $\begin{array}{l}0.006 \text { ** } \\
(0.002)\end{array}$ & $\begin{array}{l}0.006 \text { ** } \\
(0.002)\end{array}$ & $\begin{array}{l}0.007 \text { *** } \\
(0.002)\end{array}$ \\
\hline $\mathrm{ECM}^{*}$ & $\begin{array}{l}\text { log[per capitaRGDP(importer) / } \\
\text { per capitaRGDP(exporter)] }\end{array}$ & & & $\begin{array}{r}-0.001 \\
(0.002)\end{array}$ & $\begin{array}{r}0.000 \\
(0.002)\end{array}$ & $\begin{array}{r}-0.002 \\
(0.004)\end{array}$ & $\begin{array}{r}0.004 \\
(0.006)\end{array}$ & $\begin{array}{r}0.007 \\
(0.005)\end{array}$ & $\begin{array}{r}0.006 \\
(0.006)\end{array}$ & $\begin{array}{r}0.003 \\
(0.006)\end{array}$ \\
\hline $\mathrm{ECM}^{*}$ & tariff levels & & & & $\begin{array}{r}0.006 \\
(0.020)\end{array}$ & $\begin{array}{r}-0.001 \\
(0.022)\end{array}$ & $\begin{array}{r}-0.003 \\
(0.033)\end{array}$ & $\begin{array}{r}0.006 \\
(0.032)\end{array}$ & $\begin{array}{r}0.003 \\
(0.033)\end{array}$ & $\begin{array}{r}-0.013 \\
(0.034)\end{array}$ \\
\hline $\mathrm{ECM}^{*}$ & ${ }^{*} \log$ distance & & & & $\begin{array}{r}0.010 \\
(0.008)\end{array}$ & $\begin{array}{r}0.010 \\
(0.008)\end{array}$ & $\begin{array}{r}0.016 \\
(0.010)\end{array}$ & $\begin{array}{r}0.012 \\
(0.010)\end{array}$ & $\begin{array}{r}0.013 \\
(0.011)\end{array}$ & $\begin{array}{r}0.013 \\
(0.011)\end{array}$ \\
\hline $\mathrm{ECM}^{*}$ & * $\log [$ RGDP(importer)/RGDP(exporter)] & & & & & $\begin{array}{r}0.002 \\
(0.003)\end{array}$ & $\begin{array}{r}-0.004 \\
(0.004)\end{array}$ & $\begin{array}{c}-0.008 * \\
(0.004)\end{array}$ & $\begin{array}{r}-0.007 \\
(0.005)\end{array}$ & $\begin{array}{r}-0.004 \\
(0.005)\end{array}$ \\
\hline $\mathrm{ECM}^{*}$ & * log real wage $(\$)$ & & & & & & $\begin{array}{r}0.001 \\
(0.007)\end{array}$ & $\begin{array}{r}-0.007 \\
(0.007)\end{array}$ & $\begin{array}{r}-0.007 \\
(0.008)\end{array}$ & $\begin{array}{r}-0.005 \\
(0.008)\end{array}$ \\
\hline $\mathrm{ECM}^{*}$ & * long term inflation & & & & & & & $\begin{array}{r}-0.065 \\
(0.045)\end{array}$ & $\begin{array}{r}-0.066 \\
(0.045)\end{array}$ & $\begin{array}{r}-0.057 \\
(0.047)\end{array}$ \\
\hline ECM* & * long term exchange rate variability & & & & & & & $\begin{array}{r}-0.112 \\
(0.077)\end{array}$ & $\begin{array}{r}-0.109 \\
(0.078)\end{array}$ & \\
\hline ECM* & * US Importer dummy & & & & & & & & $\begin{array}{r}-0.040 \\
(0.038)\end{array}$ & $\begin{array}{r}-0.054 \\
(0.037)\end{array}$ \\
\hline & \# of Observations & 3776 & 3776 & 3776 & 3520 & 3520 & 2351 & 2323 & 2323 & 2351 \\
\hline & Adjusted R-squared & 0.251 & 0.266 & 0.288 & 0.306 & 0.307 & 0.268 & 0.244 & 0.244 & 0.288 \\
\hline & Country dummies & yes & yes & yes & yes & yes & yes & yes & yes & yes \\
\hline & Product dummies & yes & yes & yes & yes & yes & yes & yes & yes & yes \\
\hline
\end{tabular}


Appendix Table 2: Pass-through to Local Competitor Prices: Restricted Sample

\begin{tabular}{|c|c|c|c|c|c|c|c|c|c|c|}
\hline change in & exchange rate & $\begin{array}{c}\text { Eqn 1 } \\
0.543 * * * \\
(0.027)\end{array}$ & $\begin{array}{c}\text { Eqn2 } \\
0.917 * * * \\
(0.062)\end{array}$ & $\begin{array}{c}\text { Eqn3 } \\
0.858 * * * \\
(0.060)\end{array}$ & $\begin{array}{c}\text { Eqn 4 } \\
1.530 * * \\
(0.666)\end{array}$ & $\begin{array}{c}\text { Eqn 5 } \\
1.989 * * * \\
(0.672)\end{array}$ & $\begin{array}{r}\text { Eqn 6 } \\
0.446 \\
(0.910)\end{array}$ & $\begin{array}{r}\text { Eqn } 7 \\
-0.213 \\
(0.976)\end{array}$ & $\begin{array}{r}\text { Eqn 8 } \\
-0.395 \\
(0.999)\end{array}$ & $\begin{array}{c}\text { Eqn 9 } \\
-0.168 \\
(0.966)\end{array}$ \\
\hline change in & exporter's price & $\begin{array}{r}0.040 \\
(0.028)\end{array}$ & $\begin{array}{r}0.040 \\
(0.028)\end{array}$ & $\begin{array}{l}0.049 * \\
(0.027)\end{array}$ & $\begin{array}{r}0.034 \\
(0.029)\end{array}$ & $\begin{array}{r}0.032 \\
(0.029)\end{array}$ & $\begin{array}{r}0.044 \\
(0.036)\end{array}$ & $\begin{array}{l}0.057 * \\
(0.033)\end{array}$ & $\begin{array}{l}0.056 * \\
(0.033)\end{array}$ & $\begin{array}{r}0.042 \\
(0.035)\end{array}$ \\
\hline$($ delta s)* & trend & & $\begin{array}{l}-0.057^{* * *} \\
(0.009)\end{array}$ & $\begin{array}{l}-0.062 * * * \\
(0.008)\end{array}$ & $\begin{array}{l}-0.061^{* * *} \\
(0.008)\end{array}$ & $\begin{array}{l}-0.062 * * * \\
(0.008)\end{array}$ & $\begin{array}{l}-0.087^{* * *} \\
(0.010)\end{array}$ & $\begin{array}{l}-0.060 * * * \\
(0.010)\end{array}$ & $\begin{array}{l}-0.060^{* * *} \\
(0.010)\end{array}$ & $\begin{array}{l}-0.072^{* * *} \\
(0.011)\end{array}$ \\
\hline$($ delta s)* & $\begin{array}{l}\log [\text { per capitaRGDP(importer) / } \\
\text { per capitaRGDP(exporter)] }\end{array}$ & & & $\begin{array}{l}-0.033^{* * *} \\
(0.006)\end{array}$ & $\begin{array}{l}-0.033^{* * *} \\
(0.006)\end{array}$ & $\begin{array}{l}-0.073^{* * *} \\
(0.019)\end{array}$ & $\begin{array}{l}-0.090^{* * *} \\
(0.025)\end{array}$ & $\begin{array}{l}-0.108 * * * \\
(0.024)\end{array}$ & $\begin{array}{l}-0.101 * * * \\
(0.025)\end{array}$ & $\begin{array}{l}-0.073^{* * *} \\
(0.025)\end{array}$ \\
\hline$($ delta s)* & tariff levels & & & & $\begin{array}{l}-0.191 * \\
(0.114)\end{array}$ & $\begin{array}{l}-0.298 * * \\
(0.118)\end{array}$ & $\begin{array}{r}-0.065 \\
(0.152)\end{array}$ & $\begin{array}{r}0.086 \\
(0.155)\end{array}$ & $\begin{array}{r}0.117 \\
(0.160)\end{array}$ & $\begin{array}{r}0.017 \\
(0.164)\end{array}$ \\
\hline$($ delta s)* & $\log$ distance & & & & $\begin{array}{r}0.029 \\
(0.038)\end{array}$ & $\begin{array}{r}0.044 \\
(0.038)\end{array}$ & $\begin{array}{l}0.108 \text { ** } \\
(0.053)\end{array}$ & $\begin{array}{r}0.078 \\
(0.052)\end{array}$ & $\begin{array}{r}0.080 \\
(0.052)\end{array}$ & $\begin{array}{l}0.109 \text { ** } \\
(0.054)\end{array}$ \\
\hline$($ delta s)* & $\log [$ RGDP(importer)/RGDP(exporter)] & & & & & $\begin{array}{l}0.042 \text { ** } \\
(0.018)\end{array}$ & $\begin{array}{l}0.065^{* * *} \\
(0.023)\end{array}$ & $\begin{array}{l}0.088^{* * *} \\
(0.023)\end{array}$ & $\begin{array}{l}0.079^{* * *} \\
(0.025)\end{array}$ & $\begin{array}{l}0.049 \text { ** } \\
(0.024)\end{array}$ \\
\hline$($ delta s)* & log real wage $(\$)$ & & & & & & $\begin{array}{l}-0.016 * \\
(0.009)\end{array}$ & $\begin{array}{r}-0.009 \\
(0.008)\end{array}$ & $\begin{array}{r}-0.008 \\
(0.008)\end{array}$ & $\begin{array}{r}-0.013 \\
(0.009)\end{array}$ \\
\hline$($ delta s)* & long term inflation & & & & & & & $\begin{array}{l}0.437 \text { ** } \\
(0.191)\end{array}$ & $\begin{array}{l}0.436 \text { ** } \\
(0.190)\end{array}$ & $\begin{array}{l}0.178^{* * *} \\
(0.064)\end{array}$ \\
\hline$($ delta s)* & long term exchange rate variability & & & & & & & $\begin{array}{r}-1.186 \\
(1.193)\end{array}$ & $\begin{array}{r}-1.166 \\
(1.192)\end{array}$ & \\
\hline$($ delta s)* & US Importer dummy & & & & & & & & $\begin{array}{l}0.449 * * \\
(0.176)\end{array}$ & $\begin{array}{l}0.477^{* * *} \\
(0.178)\end{array}$ \\
\hline & Error Correction term (ECM) & $\begin{array}{l}-0.035 * * * \\
(0.006)\end{array}$ & $\begin{array}{l}-0.040 * * \\
(0.016)\end{array}$ & $\begin{array}{l}-0.042 * * * \\
(0.015)\end{array}$ & $\begin{array}{r}-0.016 \\
(0.085)\end{array}$ & $\begin{array}{r}0.114 \\
(0.091)\end{array}$ & $\begin{array}{r}0.078 \\
(0.135)\end{array}$ & $\begin{array}{r}0.065 \\
(0.121)\end{array}$ & $\begin{array}{r}0.062 \\
(0.123)\end{array}$ & $\begin{array}{r}0.127 \\
(0.131)\end{array}$ \\
\hline $\mathrm{ECM}^{*}$ & * trend & & $\begin{array}{r}0.001 \\
(0.002)\end{array}$ & $\begin{array}{r}0.000 \\
(0.002)\end{array}$ & $\begin{array}{r}0.001 \\
(0.002)\end{array}$ & $\begin{array}{r}0.001 \\
(0.002)\end{array}$ & $\begin{array}{r}0.000 \\
(0.002)\end{array}$ & $\begin{array}{r}-0.001 \\
(0.002)\end{array}$ & $\begin{array}{l}-0.001 \\
(0.002)\end{array}$ & $\begin{array}{r}0.000 \\
(0.002)\end{array}$ \\
\hline $\mathrm{ECM}^{*}$ & $\begin{array}{l}{ }^{*} \log [\text { per capitaRGDP(importer) / } \\
\text { per capitaRGDP(exporter)] }\end{array}$ & & & $\begin{array}{l}-0.006 * * * \\
(0.002)\end{array}$ & $\begin{array}{l}-0.007 * * * \\
(0.002)\end{array}$ & $\begin{array}{l}-0.017^{* * *} \\
(0.004)\end{array}$ & $\begin{array}{l}-0.022 * * * \\
(0.005)\end{array}$ & $\begin{array}{l}-0.017 * * * \\
(0.005)\end{array}$ & $\begin{array}{l}-0.017^{* * *} \\
(0.005)\end{array}$ & $\begin{array}{l}-0.022^{* * *} \\
(0.005)\end{array}$ \\
\hline $\mathrm{ECM}^{*}$ & * tariff levels & & & & $\begin{array}{r}-0.011 \\
(0.013)\end{array}$ & $\begin{array}{l}-0.030 \text { ** } \\
(0.014)\end{array}$ & $\begin{array}{r}-0.037 \\
(0.025)\end{array}$ & $\begin{array}{r}-0.034 \\
(0.023)\end{array}$ & $\begin{array}{r}-0.033 \\
(0.023)\end{array}$ & $\begin{array}{l}-0.044 * \\
(0.025)\end{array}$ \\
\hline $\mathrm{ECM}^{*}$ & * log distance & & & & $\begin{array}{r}0.002 \\
(0.007)\end{array}$ & $\begin{array}{r}0.001 \\
(0.007)\end{array}$ & $\begin{array}{c}0.015 * \\
(0.008)\end{array}$ & $\begin{array}{c}0.014 * \\
(0.008)\end{array}$ & $\begin{array}{c}0.014 * \\
(0.008)\end{array}$ & $\begin{array}{c}0.013 * \\
(0.008)\end{array}$ \\
\hline $\mathrm{ECM}^{*}$ & * $\log [$ RGDP(importer)/RGDP(exporter)] & & & & & $\begin{array}{l}0.0111^{* * *} \\
(0.003)^{2}\end{array}$ & $\begin{array}{l}0.014 * * * \\
(0.004)\end{array}$ & $\begin{array}{l}0.009 * * \\
(0.004)\end{array}$ & $\begin{array}{l}0.009 * * \\
(0.005)\end{array}$ & $\begin{array}{l}0.014^{* * *} \\
(0.005)\end{array}$ \\
\hline $\mathrm{ECM}^{*}$ & * log real wage $(\$)$ & & & & & & $\begin{array}{l}0.013 \text { ** } \\
(0.006)\end{array}$ & $\begin{array}{r}0.005 \\
(0.006)\end{array}$ & $\begin{array}{r}0.005 \\
(0.006)\end{array}$ & $\begin{array}{r}0.009 \\
(0.006)\end{array}$ \\
\hline $\mathrm{ECM}^{*}$ & * long term inflation & & & & & & & $\begin{array}{r}-0.045 \\
(0.029)\end{array}$ & $\begin{array}{r}-0.044 \\
(0.029)\end{array}$ & $\begin{array}{r}-0.045 \\
(0.036)\end{array}$ \\
\hline $\mathrm{ECM}^{*}$ & * long term exchange rate variability & & & & & & & $\begin{array}{l}-0.145 * * \\
(0.063)\end{array}$ & $\begin{array}{l}-0.146 * * \\
(0.063)\end{array}$ & \\
\hline $\mathrm{ECM}^{*}$ & * US Importer dummy & & & & & & & & $\begin{array}{r}0.019 \\
(0.030)\end{array}$ & $\begin{array}{r}-0.003 \\
(0.030)\end{array}$ \\
\hline & \# of Observations & 3796 & 3796 & 3796 & 3480 & 3480 & 2350 & 2326 & 2326 & 2350 \\
\hline & Adjusted R-squared & 0.207 & 0.228 & 0.243 & 0.256 & 0.261 & 0.256 & 0.282 & 0.282 & 0.264 \\
\hline & Country dummies & yes & yes & yes & yes & yes & yes & yes & yes & yes \\
\hline & Product dummies & yes & yes & yes & yes & yes & yes & yes & yes & yes \\
\hline
\end{tabular}


Appendix Table 3: Pass-through to the CPI: Restricted Sample

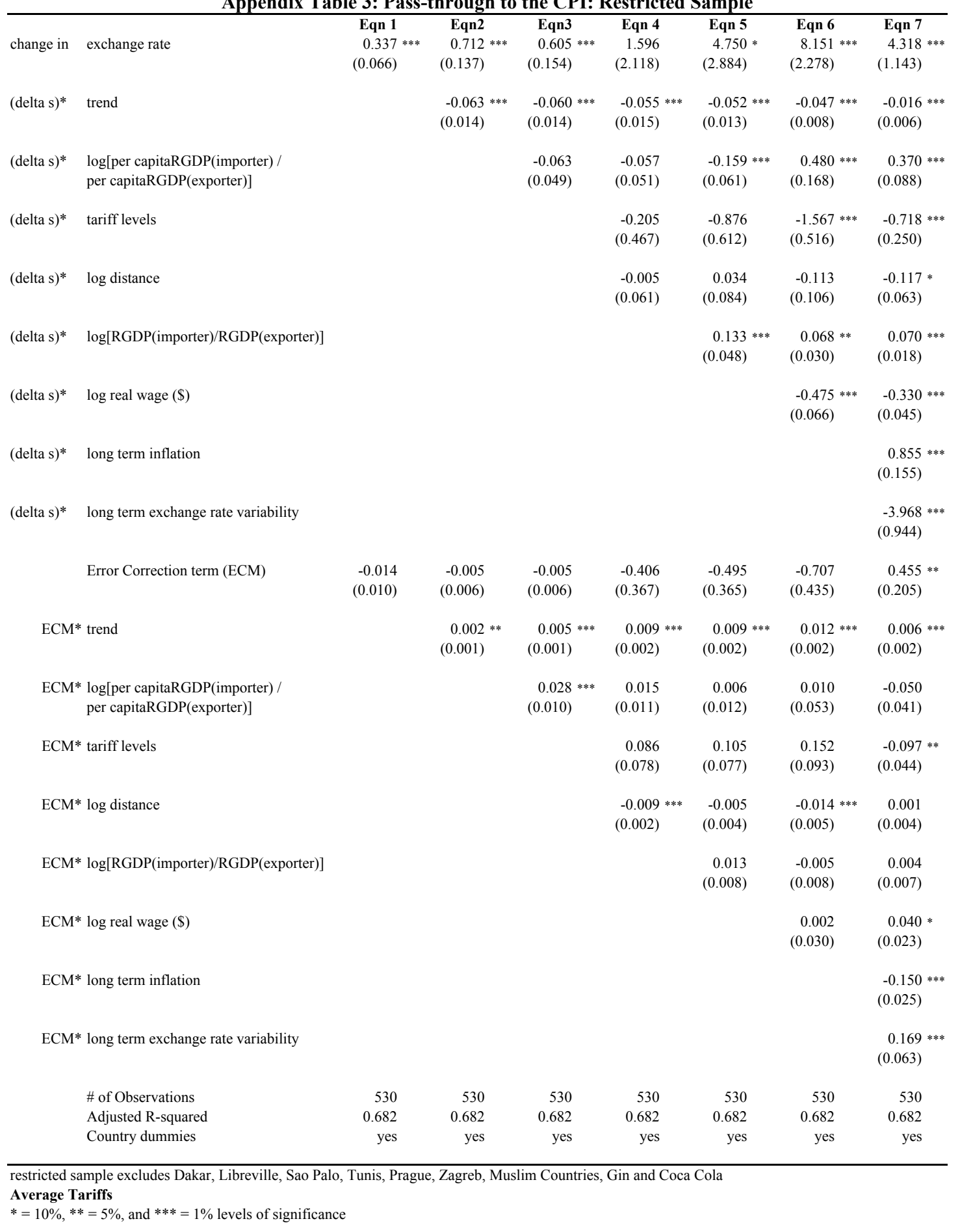


Appendix Table 4: Pass-through to Unit Values: Restricted Sample

\begin{tabular}{|c|c|c|c|c|c|c|c|c|c|c|}
\hline & & Eqn 1 & Eqn2 & Eqn3 & Eqn 4 & Eqn 5 & Eqn 6 & Eqn 7 & Eqn 8 & Eqn 9 \\
\hline change in & exchange rate & $\begin{array}{l}0.529 \text { *** } \\
(0.030)\end{array}$ & $\begin{array}{l}0.818^{* * *} \\
(0.080)\end{array}$ & $\begin{array}{l}0.774 \text { *** } \\
(0.079)\end{array}$ & $\begin{array}{r}0.733 \\
(0.719)\end{array}$ & $\begin{array}{r}1.205 \\
(0.784)\end{array}$ & $\begin{array}{c}-0.116 \\
(1.035)\end{array}$ & $\begin{array}{r}-0.823 \\
(0.948)\end{array}$ & $\begin{array}{l}-1.060 \\
(0.970)\end{array}$ & $\begin{array}{l}-1.272 \\
(0.969)\end{array}$ \\
\hline change in & exporter's price & $\begin{array}{l}0.110 \text { *** } \\
(0.031)\end{array}$ & $\begin{array}{l}0.112^{* * *} \\
(0.031)\end{array}$ & $\begin{array}{l}0.105^{* * *} \\
(0.031)\end{array}$ & $\begin{array}{l}0.099^{* * *} \\
(0.033)\end{array}$ & $\begin{array}{l}0.101 \text { *** } \\
(0.033)\end{array}$ & $\begin{array}{l}0.112 \text { *** } \\
(0.038)\end{array}$ & $\begin{array}{l}0.115^{* * *} \\
(0.035)\end{array}$ & $\begin{array}{l}0.117^{* * *} \\
(0.035)\end{array}$ & $\begin{array}{l}0.107 * * * \\
(0.037)\end{array}$ \\
\hline$(\text { delta } s)^{*}$ & trend & & $\begin{array}{l}-0.038^{* * *} \\
(0.010)\end{array}$ & $\begin{array}{l}-0.049^{* * *} \\
(0.010)\end{array}$ & $\begin{array}{l}-0.047^{* * *} \\
(0.010)\end{array}$ & $\begin{array}{l}-0.047^{* * *} \\
(0.010)\end{array}$ & $\begin{array}{l}-0.073^{* * *} \\
(0.011)\end{array}$ & $\begin{array}{l}-0.057^{* * *} \\
(0.011)\end{array}$ & $\begin{array}{l}-0.058^{* * *} \\
(0.011)\end{array}$ & $\begin{array}{l}-0.058^{* * *} \\
(0.011)\end{array}$ \\
\hline$($ delta s)* & $\begin{array}{l}\log [\text { per capitaRGDP(importer) / } \\
\text { per capitaRGDP(exporter)] }\end{array}$ & & & $\begin{array}{l}-0.043^{* * *} \\
(0.006)\end{array}$ & $\begin{array}{l}-0.042 \text { *** } \\
(0.006)\end{array}$ & $\begin{array}{l}-0.072 \text { *** } \\
(0.022)\end{array}$ & $\begin{array}{l}-0.100 \text { *** } \\
(0.025)\end{array}$ & $\begin{array}{l}-0.103 \text { *** } \\
(0.027)\end{array}$ & $\begin{array}{l}-0.094 \text { *** } \\
(0.028)\end{array}$ & $\begin{array}{l}-0.076 \text { *** } \\
(0.024)\end{array}$ \\
\hline$($ delta s)* & tariff levels & & & & $\begin{array}{r}-0.014 \\
(0.128)\end{array}$ & $\begin{array}{r}-0.107 \\
(0.145)\end{array}$ & $\begin{array}{r}0.097 \\
(0.170)\end{array}$ & $\begin{array}{r}0.116 \\
(0.163)\end{array}$ & $\begin{array}{r}0.154 \\
(0.167)\end{array}$ & $\begin{array}{r}0.168 \\
(0.168)\end{array}$ \\
\hline$($ delta s)* & log distance & & & & $\begin{array}{r}0.013 \\
(0.041)\end{array}$ & $\begin{array}{r}0.015 \\
(0.041)\end{array}$ & $\begin{array}{r}0.069 \\
(0.057)\end{array}$ & $\begin{array}{l}0.114 \text { ** } \\
(0.054)\end{array}$ & $\begin{array}{l}0.117 \text { ** } \\
(0.054)\end{array}$ & $\begin{array}{l}0.130 \text { *** } \\
(0.050)\end{array}$ \\
\hline$($ delta s)* & $\log [$ RGDP(importer)/RGDP(exporter)] & & & & & $\begin{array}{r}0.032 \\
(0.022)\end{array}$ & $\begin{array}{l}0.060 \text { ** } \\
(0.024)\end{array}$ & $\begin{array}{l}0.080 \text { *** } \\
(0.026)\end{array}$ & $\begin{array}{l}0.068 \text { ** } \\
(0.028)\end{array}$ & $\begin{array}{c}0.053 \text { ** } \\
(0.024)\end{array}$ \\
\hline$(\text { delta } \mathrm{s})^{*}$ & log real wage $(\$)$ & & & & & & $\begin{array}{r}-0.008 \\
(0.009)\end{array}$ & $\begin{array}{r}0.000 \\
(0.008)\end{array}$ & $\begin{array}{r}0.001 \\
(0.008)\end{array}$ & $\begin{array}{r}-0.004 \\
(0.008)\end{array}$ \\
\hline$($ delta s)* & long term inflation & & & & & & & $\begin{array}{l}0.764^{* * *} \\
(0.197)\end{array}$ & $\begin{array}{l}0.762 \text { *** } \\
(0.197)\end{array}$ & $\begin{array}{l}0.660 \text { *** } \\
(0.128)\end{array}$ \\
\hline$($ delta s)* & long term exchange rate variability & & & & & & & $\begin{array}{r}-0.637 \\
(1.224)\end{array}$ & $\begin{array}{r}-0.615 \\
(1.223)\end{array}$ & \\
\hline$($ delta s)* & US Importer dummy & & & & & & & & $\begin{array}{l}0.576 * * * \\
(0.186)\end{array}$ & $\begin{array}{l}0.588 * * * \\
(0.183)\end{array}$ \\
\hline & Error Correction term (ECM) & $\begin{array}{l}-0.008 \text { ** } \\
(0.003)\end{array}$ & $\begin{array}{r}-0.007 \\
(0.006)\end{array}$ & $\begin{array}{r}-0.005 \\
(0.006)\end{array}$ & $\begin{array}{r}0.062 \\
(0.040)\end{array}$ & $\begin{array}{c}0.067 * \\
(0.041)\end{array}$ & $\begin{array}{l}0.135 \text { ** } \\
(0.057)\end{array}$ & $\begin{array}{l}0.135 \text { ** } \\
(0.056)\end{array}$ & $\begin{array}{l}0.136 \text { ** } \\
(0.056)\end{array}$ & $\begin{array}{l}0.124 \text { ** } \\
(0.057)\end{array}$ \\
\hline $\mathrm{ECM}^{*}$ & * trend & & $\begin{array}{r}0.000 \\
(0.001)\end{array}$ & $\begin{array}{r}0.000 \\
(0.001)\end{array}$ & $\begin{array}{r}0.000 \\
(0.001)\end{array}$ & $\begin{array}{r}0.000 \\
(0.001)\end{array}$ & $\begin{array}{r}-0.001 \\
(0.001)\end{array}$ & $\begin{array}{r}-0.001 \\
(0.001)\end{array}$ & $\begin{array}{r}-0.001 \\
(0.001)\end{array}$ & $\begin{array}{r}-0.001 \\
(0.001)\end{array}$ \\
\hline $\mathrm{ECM}^{*}$ & $\begin{array}{l}* \log [\text { per capitaRGDP(importer) } / \\
\text { per capitaRGDP(exporter)] }\end{array}$ & & & $\begin{array}{l}0.002 * * \\
(0.001)\end{array}$ & $\begin{array}{l}0.002 * \\
(0.001)\end{array}$ & $\begin{array}{r}0.001 \\
(0.002)\end{array}$ & $\begin{array}{r}0.004 \\
(0.002)\end{array}$ & $\begin{array}{r}0.002 \\
(0.002)\end{array}$ & $\begin{array}{r}0.002 \\
(0.002)\end{array}$ & $\begin{array}{r}0.004 \\
(0.002)\end{array}$ \\
\hline $\mathrm{ECM}^{*}$ & * tariff levels & & & & $\begin{array}{r}-0.001 \\
(0.007)\end{array}$ & $\begin{array}{r}-0.002 \\
(0.007)\end{array}$ & $\begin{array}{l}-0.021 * * \\
(0.010)\end{array}$ & $\begin{array}{l}-0.021 * * \\
(0.010)\end{array}$ & $\begin{array}{l}-0.021 * * \\
(0.010)\end{array}$ & $\begin{array}{l}-0.019 * * \\
(0.010)\end{array}$ \\
\hline ECM* & * log distance & & & & $\begin{array}{l}-0.007^{* * *} \\
(0.002)\end{array}$ & $\begin{array}{l}-0.007 * * * \\
(0.003)\end{array}$ & $\begin{array}{l}-0.006 * * \\
(0.003)\end{array}$ & $\begin{array}{l}-0.006 * \\
(0.003)\end{array}$ & $\begin{array}{l}-0.006 * \\
(0.003)\end{array}$ & $\begin{array}{l}-0.006 * \\
(0.003)\end{array}$ \\
\hline ECM* & * $\log [$ RGDP(importer)/RGDP(exporter)] & & & & & $\begin{array}{r}0.000 \\
(0.002)\end{array}$ & $\begin{array}{r}0.000 \\
(0.002)\end{array}$ & $\begin{array}{r}0.000 \\
(0.002)\end{array}$ & $\begin{array}{r}0.000 \\
(0.002)\end{array}$ & $\begin{array}{r}0.000 \\
(0.002)\end{array}$ \\
\hline ECM* & * log real wage $(\$)$ & & & & & & $\begin{array}{l}-0.008^{* * *} \\
(0.003)\end{array}$ & $\begin{array}{l}-0.007 * * \\
(0.003)\end{array}$ & $\begin{array}{l}-0.007 * * \\
(0.003)\end{array}$ & $\begin{array}{l}-0.008 * * \\
(0.003)\end{array}$ \\
\hline ECM* & * long term inflation & & & & & & & $\begin{array}{r}-0.007 \\
(0.013)\end{array}$ & $\begin{array}{r}-0.007 \\
(0.013)\end{array}$ & $\begin{array}{r}-0.018 \\
(0.016)\end{array}$ \\
\hline $\mathrm{ECM}^{*}$ & * long term exchange rate variability & & & & & & & $\begin{array}{r}0.029 \\
(0.030)\end{array}$ & $\begin{array}{r}0.028 \\
(0.030)\end{array}$ & \\
\hline $\mathrm{ECM}^{*}$ & * US Importer dummy & & & & & & & & $\begin{array}{r}-0.043 \\
(0.032)\end{array}$ & $\begin{array}{r}-0.038 \\
(0.032)\end{array}$ \\
\hline & \# of Observations & 2853 & 2853 & 2853 & 2640 & 2640 & 1945 & 1934 & 1934 & 1945 \\
\hline & Adjusted R-squared & 0.196 & 0.205 & 0.229 & 0.241 & 0.242 & 0.182 & 0.226 & 0.227 & 0.215 \\
\hline & Country dummies & yes & yes & yes & yes & yes & yes & yes & yes & yes \\
\hline & Product dummies & yes & yes & yes & yes & yes & yes & yes & yes & yes \\
\hline
\end{tabular}




\section{Appendix Table 5: Pass-through to Imported Good Prices}

\begin{tabular}{|c|c|c|c|c|c|c|c|c|c|c|}
\hline change in & exchange rate & $\begin{array}{c}\text { Eqn 1 } \\
0.581 * * * \\
(0.026)\end{array}$ & $\begin{array}{c}\text { Eqn2 } \\
0.865 * * * \\
(0.061)\end{array}$ & $\begin{array}{c}\text { Eqn3 } \\
0.7666^{* * *} \\
(0.063)\end{array}$ & $\begin{array}{c}\text { Eqn } 4 \\
2.459 * * * \\
(0.926)\end{array}$ & $\begin{array}{c}\text { Eqn 5 } \\
2.6144^{* * *} \\
(0.914)\end{array}$ & $\begin{array}{c}\text { Eqn 6 } \\
2.061 * * \\
(0.978)\end{array}$ & $\begin{array}{r}\text { Eqn } 7 \\
0.767 \\
(0.826)\end{array}$ & $\begin{array}{r}\text { Eqn 8 } \\
0.802 \\
(0.835)\end{array}$ & $\begin{array}{r}\text { Eqn 9 } \\
1.352 \\
(0.886)\end{array}$ \\
\hline change in & exporter's price & $\begin{array}{l}0.0811^{* * *} \\
(0.024)^{2}\end{array}$ & $\begin{array}{l}0.100^{* * *} \\
(0.025)^{2}\end{array}$ & $\begin{array}{l}0.103^{* * *} \\
(0.024)^{*}\end{array}$ & $\begin{array}{l}0.104^{* * *} \\
(0.026)\end{array}$ & $\begin{array}{l}0.103^{* * *} \\
(0.026)\end{array}$ & $\begin{array}{l}0.069^{* *} \\
(0.030)\end{array}$ & $\begin{array}{l}0.052 * \\
(0.029)\end{array}$ & $\begin{array}{l}0.053 * \\
(0.029)\end{array}$ & $\begin{array}{l}0.062 * * \\
(0.029)\end{array}$ \\
\hline$($ delta s)* & trend & & $\begin{array}{l}-0.044^{* * *} \\
(0.008)\end{array}$ & $\begin{array}{l}-0.046^{* * *} \\
(0.008)\end{array}$ & $\begin{array}{l}-0.046 * * * \\
(0.008)\end{array}$ & $\begin{array}{l}-0.047^{* * *} \\
(0.008)\end{array}$ & $\begin{array}{l}-0.057 * * * \\
(0.010)\end{array}$ & $\begin{array}{l}-0.034^{* * *} \\
(0.010)\end{array}$ & $\begin{array}{l}-0.034^{* * *} \\
(0.010)\end{array}$ & $\begin{array}{l}-0.028^{* * *} \\
(0.010)\end{array}$ \\
\hline$($ delta s)* & $\begin{array}{l}\log [\text { per capitaRGDP(importer) / } \\
\text { per capitaRGDP(exporter)] }\end{array}$ & & & $\begin{array}{l}-0.048 * * * \\
(0.008)\end{array}$ & $\begin{array}{l}-0.048 * * * \\
(0.009)\end{array}$ & $\begin{array}{l}-0.067^{* * *} \\
(0.016)\end{array}$ & $\begin{array}{l}-0.070 * * * \\
(0.023)\end{array}$ & $\begin{array}{r}-0.019 \\
(0.022)\end{array}$ & $\begin{array}{r}-0.020 \\
(0.022)\end{array}$ & $\begin{array}{l}-0.049^{* *} \\
(0.023)\end{array}$ \\
\hline$($ delta s)* & tariff levels & & & & $\begin{array}{r}-0.179 \\
(0.173)\end{array}$ & $\begin{array}{r}-0.215 \\
(0.171)\end{array}$ & $\begin{array}{r}-0.066 \\
(0.157)\end{array}$ & $\begin{array}{r}0.107 \\
(0.138)\end{array}$ & $\begin{array}{r}0.102 \\
(0.140)\end{array}$ & $\begin{array}{r}0.007 \\
(0.142)\end{array}$ \\
\hline$($ delta s)* & $\log$ distance & & & & $\begin{array}{l}-0.092 * * \\
(0.039)\end{array}$ & $\begin{array}{l}-0.085^{* *} \\
(0.040)\end{array}$ & $\begin{array}{l}-0.101 * \\
(0.055)\end{array}$ & $\begin{array}{r}-0.083 \\
(0.052)\end{array}$ & $\begin{array}{r}-0.084 \\
(0.052)\end{array}$ & $\begin{array}{l}-0.102 * \\
(0.053)\end{array}$ \\
\hline$($ delta s)* & $\log [$ RGDP(importer)/RGDP(exporter)] & & & & & $\begin{array}{r}0.020 \\
(0.014)\end{array}$ & $\begin{array}{r}0.027 \\
(0.021)\end{array}$ & $\begin{array}{r}-0.011 \\
(0.018)\end{array}$ & $\begin{array}{r}-0.009 \\
(0.019)\end{array}$ & $\begin{array}{r}0.014 \\
(0.021)\end{array}$ \\
\hline$($ delta s)* & log real wage (\$) & & & & & & $\begin{array}{r}-0.011 \\
(0.009)\end{array}$ & $\begin{array}{r}-0.005 \\
(0.008)\end{array}$ & $\begin{array}{r}-0.005 \\
(0.008)\end{array}$ & $\begin{array}{r}-0.006 \\
(0.007)\end{array}$ \\
\hline$($ delta s)* & long term inflation & & & & & & & $\begin{array}{l}0.443^{* * *} \\
(0.157)^{* *}\end{array}$ & $\begin{array}{l}0.445 \text { *** } \\
(0.157)\end{array}$ & $\begin{array}{l}0.329 \text { *** } \\
(0.069)\end{array}$ \\
\hline$($ delta s)* & long term exchange rate variability & & & & & & & $\begin{array}{r}-1.470 \\
(0.961)\end{array}$ & $\begin{array}{r}-1.489 \\
(0.962)\end{array}$ & \\
\hline$($ delta s)* & US Importer dummy & & & & & & & & $\begin{array}{r}-0.238 \\
(0.155)\end{array}$ & $\begin{array}{l}-0.384^{* *} \\
(0.165)\end{array}$ \\
\hline & Error Correction term (ECM) & $\begin{array}{l}-0.100 \text { *** } \\
(0.007)^{*}\end{array}$ & $\begin{array}{l}-0.158 \text { *** } \\
(0.014)\end{array}$ & $\begin{array}{l}-0.157^{* * *} \\
(0.014)\end{array}$ & $\begin{array}{l}-0.2511^{* * *} \\
(0.089)^{* *}\end{array}$ & $\begin{array}{l}-0.270 \text { *** } \\
(0.089)\end{array}$ & $\begin{array}{l}-0.360 \text { *** } \\
(0.107)\end{array}$ & $\begin{array}{l}-0.240 \text { ** } \\
(0.105)\end{array}$ & $\begin{array}{l}-0.234 * * \\
(0.105)\end{array}$ & $\begin{array}{l}-0.238^{* *} \\
(0.108)\end{array}$ \\
\hline ECM* & trend & & $\begin{array}{l}0.008 \text { *** } \\
(0.002)\end{array}$ & $\begin{array}{l}0.008^{* * *} \\
(0.002)\end{array}$ & $\begin{array}{l}0.009 \text { *** } \\
(0.002)\end{array}$ & $\begin{array}{l}0.009 \text { *** } \\
(0.002)\end{array}$ & $\begin{array}{l}0.007^{* * *} \\
(0.002)\end{array}$ & $\begin{array}{l}0.005^{* *} \\
(0.002)\end{array}$ & $\begin{array}{l}0.005 \text { ** } \\
(0.002)\end{array}$ & $\begin{array}{l}0.005^{* * *} \\
(0.002)\end{array}$ \\
\hline ECM* & $\begin{array}{l}\log [\text { per capitaRGDP(importer) / } \\
\text { per capitaRGDP(exporter)] }\end{array}$ & & & $\begin{array}{r}0.000 \\
(0.002)\end{array}$ & $\begin{array}{r}0.001 \\
(0.002)\end{array}$ & $\begin{array}{r}0.003 \\
(0.003)\end{array}$ & $\begin{array}{r}0.001 \\
(0.005)\end{array}$ & $\begin{array}{r}0.003 \\
(0.005)\end{array}$ & $\begin{array}{r}0.002 \\
(0.005)\end{array}$ & $\begin{array}{r}0.000 \\
(0.005)\end{array}$ \\
\hline $\mathrm{ECM}^{*}$ & tariff levels & & & & $\begin{array}{r}-0.002 \\
(0.014)\end{array}$ & $\begin{array}{r}0.000 \\
(0.014)\end{array}$ & $\begin{array}{r}0.013 \\
(0.020)\end{array}$ & $\begin{array}{r}0.000 \\
(0.020)\end{array}$ & $\begin{array}{r}-0.002 \\
(0.020)\end{array}$ & $\begin{array}{r}-0.006 \\
(0.020)\end{array}$ \\
\hline $\mathrm{ECM}^{*}$ & log distance & & & & $\begin{array}{r}0.011 \\
(0.008)\end{array}$ & $\begin{array}{r}0.012 \\
(0.008)\end{array}$ & $\begin{array}{l}0.021^{* *} \\
(0.009)\end{array}$ & $\begin{array}{r}0.015 \\
(0.010)\end{array}$ & $\begin{array}{r}0.016 \\
(0.010)\end{array}$ & $\begin{array}{l}0.018 * \\
(0.010)\end{array}$ \\
\hline $\mathrm{ECM}^{*}$ & $\log [$ RGDP(importer)/RGDP(exporter)] & & & & & $\begin{array}{r}-0.002 \\
(0.003)\end{array}$ & $\begin{array}{r}-0.002 \\
(0.004)\end{array}$ & $\begin{array}{r}-0.004 \\
(0.004)\end{array}$ & $\begin{array}{r}-0.004 \\
(0.004)\end{array}$ & $\begin{array}{r}0.000 \\
(0.005)\end{array}$ \\
\hline $\mathrm{ECM}^{*}$ & log real wage $(\$)$ & & & & & & $\begin{array}{l}0.010 * \\
(0.006)\end{array}$ & $\begin{array}{r}-0.002 \\
(0.006)\end{array}$ & $\begin{array}{r}-0.001 \\
(0.007)\end{array}$ & $\begin{array}{r}0.002 \\
(0.007)\end{array}$ \\
\hline $\mathrm{ECM}^{*}$ & long term inflation & & & & & & & $\begin{array}{l}-0.081 * \\
(0.046)\end{array}$ & $\begin{array}{l}-0.082 * \\
(0.046)\end{array}$ & $\begin{array}{l}-0.091 * \\
(0.047)\end{array}$ \\
\hline $\mathrm{ECM}^{*}$ & long term exchange rate variability & & & & & & & $\begin{array}{l}-0.248^{* * *} \\
(0.071)\end{array}$ & $\begin{array}{l}-0.245^{* * *} \\
(0.071)\end{array}$ & \\
\hline $\mathrm{ECM}^{*}$ & US Importer dummy & & & & & & & & $\begin{array}{r}-0.049 \\
(0.035)\end{array}$ & $\begin{array}{l}-0.065 * \\
(0.036)\end{array}$ \\
\hline & \# of Observations & 5677 & 5677 & 5677 & 5192 & 5192 & 3316 & 3250 & 3250 & 3316 \\
\hline & Adjusted R-squared & 0.246 & 0.263 & 0.275 & 0.285 & 0.286 & 0.264 & 0.263 & 0.262 & 0.293 \\
\hline & Country dummies & no & no & no & no & no & no & no & no & no \\
\hline & Product dummies & yes & yes & yes & yes & yes & yes & yes & yes & yes \\
\hline
\end{tabular}




\section{Appendix Table 6: Pass-through to Local Competitor Prices}

\begin{tabular}{|c|c|c|c|c|c|c|c|c|c|c|}
\hline change in & exchange rate & $\begin{array}{c}\text { Eqn 1 } \\
0.560 \text { *** } \\
(0.025)\end{array}$ & $\begin{array}{c}\text { Eqn2 } \\
0.925 * * * \\
(0.052)\end{array}$ & $\begin{array}{c}\text { Eqn3 } \\
0.860 * * * \\
(0.052)\end{array}$ & $\begin{array}{c}\text { Eqn 4 } \\
1.227 * * \\
(0.516)\end{array}$ & $\begin{array}{c}\text { Eqn 5 } \\
1.665 * * * \\
(0.522)\end{array}$ & $\begin{array}{r}\text { Eqn } 6 \\
-0.246 \\
(0.703)\end{array}$ & $\begin{array}{r}\text { Eqn } 7 \\
-1.059 \\
(0.758)\end{array}$ & $\begin{array}{c}\text { Eqn 8 } \\
-1.171 \\
(0.768)\end{array}$ & $\begin{array}{r}\text { Eqn 9 } \\
-0.782 \\
(0.761)\end{array}$ \\
\hline change in & exporter's price & $\begin{array}{l}0.055 * * \\
(0.028)\end{array}$ & $\begin{array}{l}0.049 * \\
(0.028)\end{array}$ & $\begin{array}{l}0.051 * \\
(0.028)\end{array}$ & $\begin{array}{r}0.037 \\
(0.030)\end{array}$ & $\begin{array}{r}0.034 \\
(0.030)\end{array}$ & $\begin{array}{r}0.034 \\
(0.038)\end{array}$ & $\begin{array}{r}0.031 \\
(0.036)\end{array}$ & $\begin{array}{r}0.030 \\
(0.036)\end{array}$ & $\begin{array}{r}0.029 \\
(0.037)\end{array}$ \\
\hline$($ delta s)* & trend & & $\begin{array}{l}-0.056 * * * \\
(0.007)\end{array}$ & $\begin{array}{l}-0.057^{* * *} \\
(0.007)\end{array}$ & $\begin{array}{l}-0.057^{* * *} \\
(0.007)\end{array}$ & $\begin{array}{l}-0.059 * * * \\
(0.007)\end{array}$ & $\begin{array}{l}-0.085^{* * *} \\
(0.009)\end{array}$ & $\begin{array}{l}-0.059^{* * *} \\
(0.011)\end{array}$ & $\begin{array}{l}-0.059 * * * \\
(0.011)\end{array}$ & $\begin{array}{l}-0.069^{* * *} \\
(0.011)\end{array}$ \\
\hline$($ delta s)* & $\begin{array}{l}\log [\text { per capitaRGDP(importer) / } \\
\text { per capitaRGDP(exporter)] }\end{array}$ & & & $\begin{array}{l}-0.031^{* * *} \\
(0.006)\end{array}$ & $\begin{array}{l}-0.032 * * * \\
(0.006)\end{array}$ & $\begin{array}{l}-0.078 * * * \\
(0.015)\end{array}$ & $\begin{array}{l}-0.079^{* * *} \\
(0.024)^{2}\end{array}$ & $\begin{array}{l}-0.083^{* * *} \\
(0.021)\end{array}$ & $\begin{array}{l}-0.079 * * * \\
(0.022)\end{array}$ & $\begin{array}{l}-0.063 * * \\
(0.025)\end{array}$ \\
\hline$($ delta s)* & tariff levels & & & & $\begin{array}{r}-0.131 \\
(0.087)\end{array}$ & $\begin{array}{l}-0.237^{* * *} \\
(0.089)\end{array}$ & $\begin{array}{r}0.071 \\
(0.115)\end{array}$ & $\begin{array}{l}0.243^{* *} \\
(0.121)\end{array}$ & $\begin{array}{l}0.260 * * \\
(0.123)\end{array}$ & $\begin{array}{r}0.127 \\
(0.126)\end{array}$ \\
\hline$($ delta s)* & log distance & & & & $\begin{array}{r}0.032 \\
(0.036)\end{array}$ & $\begin{array}{r}0.052 \\
(0.036)\end{array}$ & $\begin{array}{l}0.113 \text { ** } \\
(0.051)\end{array}$ & $\begin{array}{c}0.090 * \\
(0.049)\end{array}$ & $\begin{array}{c}0.092 * \\
(0.049)\end{array}$ & $\begin{array}{l}0.116 \text { ** } \\
(0.052)\end{array}$ \\
\hline$($ delta s)* & $\log [$ RGDP(importer)/RGDP(exporter)] & & & & & $\begin{array}{l}0.049^{* * *} \\
(0.015)\end{array}$ & $\begin{array}{l}0.053 \text { ** } \\
(0.022)\end{array}$ & $\begin{array}{l}0.066^{* * *} \\
(0.019)\end{array}$ & $\begin{array}{l}0.060 \text { *** } \\
(0.020)\end{array}$ & $\begin{array}{c}0.037 * \\
(0.023)\end{array}$ \\
\hline$($ delta s)* & log real wage $(\$)$ & & & & & & $\begin{array}{l}-0.021^{* * *} \\
(0.008)\end{array}$ & $\begin{array}{r}-0.009 \\
(0.007)\end{array}$ & $\begin{array}{r}-0.009 \\
(0.007)\end{array}$ & $\begin{array}{l}-0.019 * * \\
(0.008)\end{array}$ \\
\hline$($ delta s)* & long term inflation & & & & & & & $\begin{array}{l}0.531 \text { *** } \\
(0.200)\end{array}$ & $\begin{array}{l}0.527 \text { *** } \\
(0.200)\end{array}$ & $\begin{array}{l}0.193 \text { *** } \\
(0.061)\end{array}$ \\
\hline$($ delta s)* & long term exchange rate variability & & & & & & & $\begin{array}{r}-1.767 \\
(1.240)\end{array}$ & $\begin{array}{r}-1.727 \\
(1.240)\end{array}$ & \\
\hline$($ delta s)* & US Importer dummy & & & & & & & & $\begin{array}{l}0.5266^{* * *} \\
(0.166)\end{array}$ & $\begin{array}{l}0.529 * * * \\
(0.170)\end{array}$ \\
\hline & Error Correction term (ECM) & $\begin{array}{l}-0.036 * * * \\
(0.006)\end{array}$ & $\begin{array}{l}-0.030 * * \\
(0.014)\end{array}$ & $\begin{array}{l}-0.032 * * \\
(0.013)\end{array}$ & $\begin{array}{r}-0.007 \\
(0.074)\end{array}$ & $\begin{array}{r}0.079 \\
(0.075)\end{array}$ & $\begin{array}{r}0.018 \\
(0.105)\end{array}$ & $\begin{array}{r}-0.011 \\
(0.100)\end{array}$ & $\begin{array}{r}-0.012 \\
(0.100)\end{array}$ & $\begin{array}{r}0.022 \\
(0.103)\end{array}$ \\
\hline $\mathrm{ECM}^{*}$ & trend & & $\begin{array}{r}-0.001 \\
(0.002)\end{array}$ & $\begin{array}{r}-0.001 \\
(0.002)\end{array}$ & $\begin{array}{r}-0.001 \\
(0.002)\end{array}$ & $\begin{array}{r}-0.001 \\
(0.002)\end{array}$ & $\begin{array}{r}0.000 \\
(0.002)\end{array}$ & $\begin{array}{r}0.000 \\
(0.002)\end{array}$ & $\begin{array}{r}0.000 \\
(0.002)\end{array}$ & $\begin{array}{r}0.000 \\
(0.002)\end{array}$ \\
\hline $\mathrm{ECM}^{*}$ & $\begin{array}{l}\log [\text { per capitaRGDP(importer) / } \\
\text { per capitaRGDP(exporter)] }\end{array}$ & & & $\begin{array}{l}-0.004^{* *} \\
(0.002)\end{array}$ & $\begin{array}{l}-0.005^{* * *} \\
(0.002)\end{array}$ & $\begin{array}{l}-0.015^{* * *} \\
(0.003)\end{array}$ & $\begin{array}{l}-0.018^{* * *} \\
(0.005)\end{array}$ & $\begin{array}{l}-0.014^{* * *} \\
(0.005)\end{array}$ & $\begin{array}{l}-0.014 * * * \\
(0.005)\end{array}$ & $\begin{array}{l}-0.018 \text { *** } \\
(0.005)\end{array}$ \\
\hline $\mathrm{ECM}^{*}$ & tariff levels & & & & $\begin{array}{r}-0.005 \\
(0.010)\end{array}$ & $\begin{array}{l}-0.017 * \\
(0.010)\end{array}$ & $\begin{array}{r}-0.028 \\
(0.018)\end{array}$ & $\begin{array}{r}-0.024 \\
(0.017)\end{array}$ & $\begin{array}{r}-0.024 \\
(0.018)\end{array}$ & $\begin{array}{r}-0.030 \\
(0.018)\end{array}$ \\
\hline $\mathrm{ECM}^{*}$ & log distance & & & & $\begin{array}{r}0.000 \\
(0.007)\end{array}$ & $\begin{array}{r}-0.001 \\
(0.007)\end{array}$ & $\begin{array}{l}0.015 \text { ** } \\
(0.008)\end{array}$ & $\begin{array}{l}0.016 \text { ** } \\
(0.008)\end{array}$ & $\begin{array}{l}0.016 \text { ** } \\
(0.008)\end{array}$ & $\begin{array}{l}0.016^{* *} \\
(0.008)\end{array}$ \\
\hline $\mathrm{ECM}^{*}$ & $\log [$ RGDP(importer)/RGDP(exporter) $]$ & & & & & $\begin{array}{l}0.011 \text { *** } \\
(0.003)\end{array}$ & $\begin{array}{l}0.012 \text { *** } \\
(0.004)\end{array}$ & $\begin{array}{c}0.007 \text { * } \\
(0.004)\end{array}$ & $\begin{array}{r}0.007 \\
(0.004)\end{array}$ & $\begin{array}{l}0.012 \text { *** } \\
(0.005)\end{array}$ \\
\hline $\mathrm{ECM}^{*}$ & log real wage $(\$)$ & & & & & & $\begin{array}{r}0.009 \\
(0.006)\end{array}$ & $\begin{array}{r}0.007 \\
(0.006)\end{array}$ & $\begin{array}{r}0.007 \\
(0.006)\end{array}$ & $\begin{array}{l}0.011 * \\
(0.006)\end{array}$ \\
\hline $\mathrm{ECM}^{*}$ & long term inflation & & & & & & & $\begin{array}{r}0.022 \\
(0.023)\end{array}$ & $\begin{array}{r}0.023 \\
(0.023)\end{array}$ & $\begin{array}{r}0.006 \\
(0.027)\end{array}$ \\
\hline $\mathrm{ECM}^{*}$ & long term exchange rate variability & & & & & & & $\begin{array}{l}-0.237^{* * *} \\
(0.075)\end{array}$ & $\begin{array}{l}-0.239 * * * \\
(0.075)\end{array}$ & \\
\hline $\mathrm{ECM}^{*}$ & US Importer dummy & & & & & & & & $\begin{array}{r}0.028 \\
(0.029)\end{array}$ & $\begin{array}{r}0.001 \\
(0.030)\end{array}$ \\
\hline & \# of Observations & 5630 & 5630 & 5630 & 5077 & 5077 & 3299 & 3235 & 3235 & 3299 \\
\hline & Adjusted R-squared & 0.169 & 0.185 & 0.191 & 0.200 & 0.205 & 0.205 & 0.232 & 0.232 & 0.212 \\
\hline & Country dummies & no & no & no & no & no & no & no & no & no \\
\hline & Product dummies & yes & yes & yes & yes & yes & yes & yes & yes & yes \\
\hline
\end{tabular}




\section{Appendix Table 7: Pass-through to the CPI}

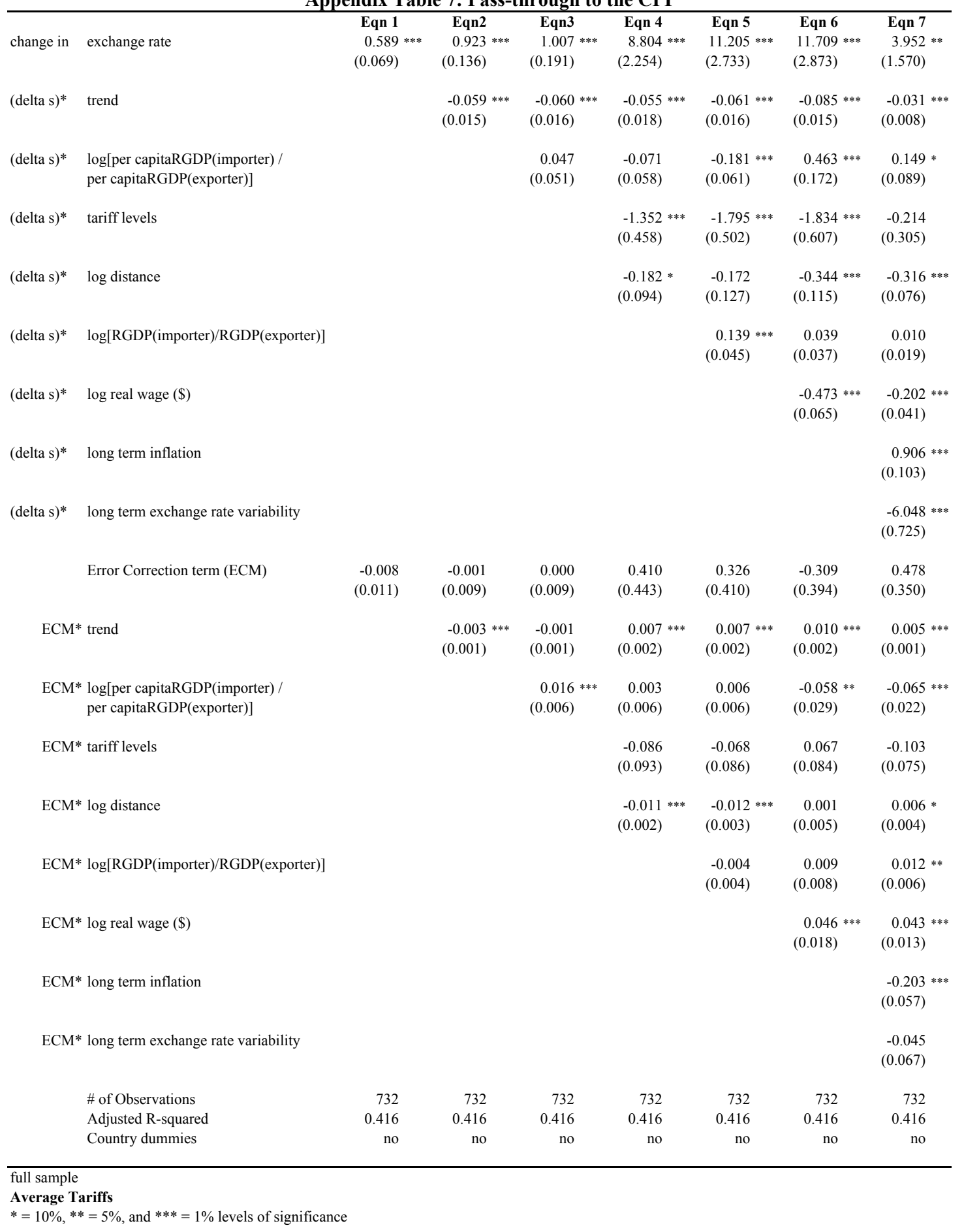


Appendix Table 8: Pass-through to Unit Values

\begin{tabular}{|c|c|c|c|c|c|c|c|c|c|c|}
\hline change in & exchange rate & $\begin{array}{c}\text { Eqn 1 } \\
0.675^{* * *} \\
(0.051)\end{array}$ & $\begin{array}{c}\text { Eqn2 } \\
0.402 * * * \\
(0.132)\end{array}$ & $\begin{array}{c}\text { Eqn3 } \\
0.425 * * * \\
(0.135)\end{array}$ & $\begin{array}{c}\text { Eqn 4 } \\
5.4522^{* * *} \\
(1.219)\end{array}$ & $\begin{array}{c}\text { Eqn 5 } \\
5.2822^{* * *} \\
(1.301)\end{array}$ & $\begin{array}{c}\text { Eqn 6 } \\
2.549 * \\
(1.546)\end{array}$ & $\begin{array}{c}\text { Eqn } 7 \\
2.486 * \\
(1.494)\end{array}$ & $\begin{array}{c}\text { Eqn 8 } \\
2.552 * \\
(1.517)\end{array}$ & $\begin{array}{c}\text { Eqn 9 } \\
2.663 * \\
(1.589)\end{array}$ \\
\hline change in & exporter's price & $\begin{array}{r}0.082 \\
(0.063)\end{array}$ & $\begin{array}{c}0.111 * \\
(0.065)\end{array}$ & $\begin{array}{c}0.114 * \\
(0.065)\end{array}$ & $\begin{array}{r}0.107 \\
(0.068)\end{array}$ & $\begin{array}{r}0.106 \\
(0.068)\end{array}$ & $\begin{array}{l}0.250 \text { *** } \\
(0.068)\end{array}$ & $\begin{array}{l}0.257 \text { *** } \\
(0.070)\end{array}$ & $\begin{array}{l}0.257 * * * \\
(0.070)\end{array}$ & $\begin{array}{l}0.2566^{* * *} \\
(0.069)\end{array}$ \\
\hline$($ delta s)* & trend & & $\begin{array}{l}0.040 \text { ** } \\
(0.018)\end{array}$ & $\begin{array}{l}0.040 \text { ** } \\
(0.017)\end{array}$ & $\begin{array}{l}0.046^{* * *} \\
(0.018)\end{array}$ & $\begin{array}{l}0.047^{* * *} \\
(0.018)\end{array}$ & $\begin{array}{l}0.045^{* *} \\
(0.020)\end{array}$ & $\begin{array}{l}0.041 * \\
(0.022)\end{array}$ & $\begin{array}{l}0.041 * \\
(0.022)\end{array}$ & $\begin{array}{l}0.039 * \\
(0.022)\end{array}$ \\
\hline$($ delta s)* & $\begin{array}{l}\log [\text { per capitaRGDP(importer) / } \\
\text { per capitaRGDP(exporter)] }\end{array}$ & & & $\begin{array}{r}0.004 \\
(0.014)\end{array}$ & $\begin{array}{r}0.016 \\
(0.013)\end{array}$ & $\begin{array}{r}0.029 \\
(0.037)\end{array}$ & $\begin{array}{l}0.085 * \\
(0.047)\end{array}$ & $\begin{array}{l}0.095 \text { ** } \\
(0.048)\end{array}$ & $\begin{array}{l}0.093 * \\
(0.049)\end{array}$ & $\begin{array}{l}0.083 * \\
(0.049)\end{array}$ \\
\hline$($ delta s)* & tariff levels & & & & $\begin{array}{l}-0.434 * \\
(0.224)\end{array}$ & $\begin{array}{l}-0.401 * \\
(0.242)\end{array}$ & $\begin{array}{r}-0.104 \\
(0.291)\end{array}$ & $\begin{array}{r}-0.096 \\
(0.299)\end{array}$ & $\begin{array}{r}-0.107 \\
(0.302)\end{array}$ & $\begin{array}{r}-0.097 \\
(0.295)\end{array}$ \\
\hline$($ delta s)* & $\log$ distance & & & & $\begin{array}{l}-0.336 * * * \\
(0.071)\end{array}$ & $\begin{array}{l}-0.338 * * * \\
(0.071)\end{array}$ & $\begin{array}{l}-0.205^{* *} \\
(0.084)\end{array}$ & $\begin{array}{l}-0.190 * * \\
(0.090)\end{array}$ & $\begin{array}{l}-0.190 * * \\
(0.090)\end{array}$ & $\begin{array}{l}-0.212 * * \\
(0.083)\end{array}$ \\
\hline$($ delta s)* & $\log [$ RGDP(importer)/RGDP(exporter)] & & & & & $\begin{array}{r}-0.014 \\
(0.036)\end{array}$ & $\begin{array}{l}-0.107 * * \\
(0.042)\end{array}$ & $\begin{array}{l}-0.114 * * * \\
(0.042)\end{array}$ & $\begin{array}{l}-0.110 * * \\
(0.044)\end{array}$ & $\begin{array}{l}-0.107^{* *} \\
(0.044)\end{array}$ \\
\hline$($ delta s)* & log real wage $(\$)$ & & & & & & $\begin{array}{r}-0.001 \\
(0.013)\end{array}$ & $\begin{array}{r}-0.012 \\
(0.015)\end{array}$ & $\begin{array}{r}-0.012 \\
(0.015)\end{array}$ & $\begin{array}{r}-0.004 \\
(0.013)\end{array}$ \\
\hline$($ delta s)* & long term inflation & & & & & & & $\begin{array}{r}0.069 \\
(0.312)\end{array}$ & $\begin{array}{r}0.073 \\
(0.313)\end{array}$ & $\begin{array}{r}-0.145 \\
(0.183)\end{array}$ \\
\hline$($ delta s)* & long term exchange rate variability & & & & & & & $\begin{array}{r}-1.504 \\
(1.810)\end{array}$ & $\begin{array}{r}-1.532 \\
(1.818)\end{array}$ & \\
\hline$($ delta s)* & US Importer dummy & & & & & & & & $\begin{array}{r}-0.232 \\
(0.267)\end{array}$ & $\begin{array}{r}-0.162 \\
(0.253)\end{array}$ \\
\hline & Error Correction term (ECM) & $\begin{array}{l}-0.180^{* * *} \\
(0.025)\end{array}$ & $\begin{array}{l}-0.155^{* * *} \\
(0.029)^{*}\end{array}$ & $\begin{array}{l}-0.159 * * * \\
(0.031)\end{array}$ & $\begin{array}{r}-0.052 \\
(0.114)\end{array}$ & $\begin{array}{r}-0.045 \\
(0.132)\end{array}$ & $\begin{array}{r}0.128 \\
(0.113)\end{array}$ & $\begin{array}{l}0.212 * \\
(0.108)\end{array}$ & $\begin{array}{l}0.209 * \\
(0.108)\end{array}$ & $\begin{array}{r}0.137 \\
(0.112)\end{array}$ \\
\hline ECM* & trend & & $\begin{array}{l}-0.004 * \\
(0.002)\end{array}$ & $\begin{array}{l}-0.004 * \\
(0.002)\end{array}$ & $\begin{array}{l}-0.004 * \\
(0.002)\end{array}$ & $\begin{array}{l}-0.004 * \\
(0.002)\end{array}$ & $\begin{array}{l}-0.005 * \\
(0.003)\end{array}$ & $\begin{array}{l}-0.006 * * \\
(0.003)\end{array}$ & $\begin{array}{l}-0.0066^{* *} \\
(0.003)\end{array}$ & $\begin{array}{l}-0.005^{* *} \\
(0.003)\end{array}$ \\
\hline $\mathrm{ECM}^{*}$ & $\begin{array}{l}\log [\text { per capitaRGDP(importer) / } \\
\text { per capitaRGDP(exporter)] }\end{array}$ & & & $\begin{array}{r}-0.004 \\
(0.003)\end{array}$ & $\begin{array}{l}-0.006 * \\
(0.003)\end{array}$ & $\begin{array}{r}-0.007 \\
(0.005)\end{array}$ & $\begin{array}{r}-0.008 \\
(0.008)\end{array}$ & $\begin{array}{r}-0.004 \\
(0.008)\end{array}$ & $\begin{array}{r}-0.004 \\
(0.008)\end{array}$ & $\begin{array}{r}-0.008 \\
(0.008)\end{array}$ \\
\hline ECM* & tariff levels & & & & $\begin{array}{r}0.021 \\
(0.023)\end{array}$ & $\begin{array}{r}0.020 \\
(0.027)\end{array}$ & $\begin{array}{r}0.016 \\
(0.021)\end{array}$ & $\begin{array}{r}-0.001 \\
(0.019)\end{array}$ & $\begin{array}{r}-0.001 \\
(0.019)\end{array}$ & $\begin{array}{r}0.014 \\
(0.021)\end{array}$ \\
\hline ECM* & log distance & & & & $\begin{array}{l}-0.025^{* * *} \\
(0.009)\end{array}$ & $\begin{array}{l}-0.025 * * * \\
(0.009)\end{array}$ & $\begin{array}{l}-0.037 \text { *** } \\
(0.014)\end{array}$ & $\begin{array}{l}-0.039^{* * *} \\
(0.015)\end{array}$ & $\begin{array}{l}-0.039 * * * \\
(0.015)\end{array}$ & $\begin{array}{l}-0.037^{* * *} \\
(0.014)\end{array}$ \\
\hline $\mathrm{ECM}^{*}$ & $\log [$ RGDP(importer)/RGDP(exporter)] & & & & & $\begin{array}{r}0.001 \\
(0.005)\end{array}$ & $\begin{array}{r}0.006 \\
(0.007)\end{array}$ & $\begin{array}{r}0.003 \\
(0.007)\end{array}$ & $\begin{array}{r}0.003 \\
(0.007)\end{array}$ & $\begin{array}{r}0.006 \\
(0.007)\end{array}$ \\
\hline ECM* & log real wage $(\$)$ & & & & & & $\begin{array}{r}0.001 \\
(0.013)\end{array}$ & $\begin{array}{r}-0.010 \\
(0.014)\end{array}$ & $\begin{array}{r}-0.010 \\
(0.014)\end{array}$ & $\begin{array}{r}-0.002 \\
(0.014)\end{array}$ \\
\hline ECM* & long term inflation & & & & & & & $\begin{array}{r}-0.014 \\
(0.051)\end{array}$ & $\begin{array}{r}-0.014 \\
(0.051)\end{array}$ & $\begin{array}{r}-0.024 \\
(0.045)\end{array}$ \\
\hline $\mathrm{ECM}^{*}$ & long term exchange rate variability & & & & & & & $\begin{array}{r}-0.093 \\
(0.103)\end{array}$ & $\begin{array}{r}-0.093 \\
(0.103)\end{array}$ & \\
\hline $\mathrm{ECM}^{*}$ & US Importer dummy & & & & & & & & $\begin{array}{r}-0.029 \\
(0.046)\end{array}$ & $\begin{array}{r}-0.049 \\
(0.043)\end{array}$ \\
\hline & \# of Observations & 3774 & 3774 & 3774 & 3548 & 3548 & 2625 & 2561 & 2561 & 2625 \\
\hline & Adjusted R-squared & 0.135 & 0.137 & 0.137 & 0.147 & 0.146 & 0.147 & 0.146 & 0.145 & 0.146 \\
\hline & Country dummies & no & no & no & no & no & no & no & no & no \\
\hline & Product dummies & yes & yes & yes & yes & yes & yes & yes & yes & yes \\
\hline
\end{tabular}

$*=10 \%, * *=5 \%$, and $* * *=1 \%$ levels of significance 
Appendix Table 9: Pass-through to Imported Good Prices

\begin{tabular}{|c|c|c|c|c|c|c|c|c|}
\hline change in & exchange rate & $\begin{array}{c}\text { Eqn 1 } \\
0.423 \\
(0.029)\end{array}$ & $\begin{array}{r}\text { Eqn2 } \\
0.010 \\
(0.061)\end{array}$ & $\begin{array}{c}\text { Eqn3 } \\
0.296 \\
(0.084)\end{array}$ & $\begin{array}{c}\text { Eqn } 4 \\
0.302 \\
(0.084)\end{array}$ & $\begin{array}{c}\text { Eqn 5 } \\
1.536 * \\
(0.859)\end{array}$ & $\begin{array}{r}\text { Eqn 6 } \\
1.159 \\
(0.824)\end{array}$ & $\begin{array}{r}\text { Eqn 7 } \\
1.116 \\
(0.826)\end{array}$ \\
\hline & depreciations & & $\begin{array}{l}0.241^{* * *} \\
(0.086)\end{array}$ & $\begin{array}{l}0.257 * * * \\
(0.086)\end{array}$ & $\begin{array}{l}0.219 \text { ** } \\
(0.088)\end{array}$ & $\begin{array}{l}0.214 * \\
(0.126)\end{array}$ & $\begin{array}{r}0.051 \\
(0.119)\end{array}$ & $\begin{array}{r}0.073 \\
(0.119)\end{array}$ \\
\hline & depreciations above $25 \%$ & & $\begin{array}{l}0.342 * * * \\
(0.052)\end{array}$ & $\begin{array}{l}0.269 * * * \\
(0.053)\end{array}$ & $\begin{array}{l}0.246 \text { *** } \\
(0.052)\end{array}$ & $\begin{array}{c}0.141 * \\
(0.086)\end{array}$ & $\begin{array}{r}0.080 \\
(0.091)\end{array}$ & $\begin{array}{r}0.071 \\
(0.091)\end{array}$ \\
\hline change in & exporter's price & $\begin{array}{l}0.086^{* * * *} \\
(0.024)\end{array}$ & $\begin{array}{l}0.070 * * * \\
(0.024)\end{array}$ & $\begin{array}{l}0.089^{* * *} \\
(0.024)\end{array}$ & $\begin{array}{l}0.092 \text { **** } \\
(0.024)\end{array}$ & $\begin{array}{l}0.060 * * \\
(0.029)\end{array}$ & $\begin{array}{l}0.049 * \\
(0.029)\end{array}$ & $\begin{array}{l}0.047 * \\
(0.029)\end{array}$ \\
\hline$($ delta s)* & trend & & & $\begin{array}{l}-0.040^{* * *} \\
(0.008)\end{array}$ & $\begin{array}{l}-0.041^{* * *} \\
(0.008)\end{array}$ & $\begin{array}{l}-0.061 * * * \\
(0.010)\end{array}$ & $\begin{array}{l}-0.049 * * * \\
(0.010)\end{array}$ & $\begin{array}{l}-0.050 * * * \\
(0.010)\end{array}$ \\
\hline$($ delta s)* & $\begin{array}{l}\log [\text { per capitaRGDP(importer) / } \\
\text { per capitaRGDP(exporter)] }\end{array}$ & & & & $\begin{array}{l}-0.024 \text { *** } \\
(0.009)\end{array}$ & $\begin{array}{l}-0.058 * * \\
(0.024)\end{array}$ & $\begin{array}{l}-0.042 * \\
(0.024)\end{array}$ & $\begin{array}{l}-0.047 * \\
(0.024)\end{array}$ \\
\hline$($ delta s)* & tariff levels & & & & & $\begin{array}{r}-0.158 \\
(0.145)\end{array}$ & $\begin{array}{r}-0.089 \\
(0.145)\end{array}$ & $\begin{array}{r}-0.086 \\
(0.146)\end{array}$ \\
\hline$($ delta s)* & log distance & & & & & $\begin{array}{r}-0.025 \\
(0.050)\end{array}$ & $\begin{array}{r}-0.017 \\
(0.050)\end{array}$ & $\begin{array}{r}-0.012 \\
(0.049)\end{array}$ \\
\hline$($ delta s)* & $\log [$ RGDP(importer)/RGDP(exporte & & & & & $\begin{array}{r}0.033 \\
(0.022)\end{array}$ & $\begin{array}{r}0.022 \\
(0.022)\end{array}$ & $\begin{array}{r}0.027 \\
(0.022)\end{array}$ \\
\hline$($ delta s)* & log real wage (\$) & & & & & $\begin{array}{r}-0.005 \\
(0.008)\end{array}$ & $\begin{array}{r}-0.004 \\
(0.008)\end{array}$ & $\begin{array}{r}-0.004 \\
(0.008)\end{array}$ \\
\hline$($ delta s)* & long term inflation & & & & & & $\begin{array}{c}0.330 * \\
(0.200)\end{array}$ & $\begin{array}{r}0.296 \\
(0.201)\end{array}$ \\
\hline$($ delta s)* & long term exchange rate variability & & & & & & $\begin{array}{r}-1.258 \\
(1.297)\end{array}$ & $\begin{array}{r}-1.344 \\
(1.292)\end{array}$ \\
\hline$($ delta s)* & US Importer dummy & & & & & & $\begin{array}{l}-0.325 \text { ** } \\
(0.164)\end{array}$ & $\begin{array}{l}-0.336 \text { ** } \\
(0.165)\end{array}$ \\
\hline$($ delta s)* & stage of business cycle & & & & & & & $\begin{array}{c}-0.677 * \\
(0.388)\end{array}$ \\
\hline & Error Correction term (ECM) & $\begin{array}{l}-0.107 * * * \\
(0.007)\end{array}$ & $\begin{array}{l}-0.105 * * * \\
(0.007)\end{array}$ & $\begin{array}{l}-0.158 * * * \\
(0.016)\end{array}$ & $\begin{array}{l}-0.158 \text { *** } \\
(0.016)\end{array}$ & $\begin{array}{l}-0.571^{* * *} \\
(0.117)\end{array}$ & $\begin{array}{l}-0.377^{* * *} \\
(0.116)\end{array}$ & $\begin{array}{l}-0.367^{* * *} \\
(0.115)\end{array}$ \\
\hline $\mathrm{ECM}^{*}$ & * trend & & & $\begin{array}{l}0.007 * * * \\
(0.002)\end{array}$ & $\begin{array}{l}0.007^{* * * *} \\
(0.002)^{2}\end{array}$ & $\begin{array}{l}0.006 * * * \\
(0.002)\end{array}$ & $\begin{array}{c}0.004 * \\
(0.002)\end{array}$ & $\begin{array}{c}0.004 * \\
(0.002)\end{array}$ \\
\hline $\mathrm{ECM}^{*}$ & $\begin{array}{l}\text { * log[per capitaRGDP(importer) / } \\
\text { per capitaRGDP(exporter)] }\end{array}$ & & & & $\begin{array}{r}0.001 \\
(0.002)\end{array}$ & $\begin{array}{r}0.000 \\
(0.006)\end{array}$ & $\begin{array}{r}-0.001 \\
(0.006)\end{array}$ & $\begin{array}{r}-0.001 \\
(0.006)\end{array}$ \\
\hline $\mathrm{ECM}^{*}$ & * tariff levels & & & & & $\begin{array}{r}0.030 \\
(0.022)\end{array}$ & $\begin{array}{r}0.005 \\
(0.021)\end{array}$ & $\begin{array}{r}0.004 \\
(0.021)\end{array}$ \\
\hline $\mathrm{ECM}^{*}$ & * log distance & & & & & $\begin{array}{l}0.038 * * * \\
(0.010)\end{array}$ & $\begin{array}{l}0.030^{* * * *} \\
(0.011)\end{array}$ & $\begin{array}{l}0.029 \text { *** } \\
(0.010)\end{array}$ \\
\hline $\mathrm{ECM}^{*}$ & * $\log [$ RGDP(importer)/RGDP(exporte & & & & & $\begin{array}{r}0.001 \\
(0.006)\end{array}$ & $\begin{array}{r}0.001 \\
(0.006)\end{array}$ & $\begin{array}{r}0.001 \\
(0.006)\end{array}$ \\
\hline $\mathrm{ECM}^{*}$ & * log real wage $(\$)$ & & & & & $\begin{array}{l}0.016 \text { ** } \\
(0.007)\end{array}$ & $\begin{array}{r}0.002 \\
(0.008)\end{array}$ & $\begin{array}{r}0.001 \\
(0.008)\end{array}$ \\
\hline ECM* & * long term inflation & & & & & & $\begin{array}{l}-0.082 * \\
(0.046)\end{array}$ & $\begin{array}{l}-0.079 * \\
(0.047)\end{array}$ \\
\hline ECM* & * long term exchange rate variability & & & & & & $\begin{array}{l}-0.175^{* *} \\
(0.072)\end{array}$ & $\begin{array}{l}-0.183^{* *} \\
(0.074)\end{array}$ \\
\hline $\mathrm{ECM}^{*}$ & * US Importer dummy & & & & & & $\begin{array}{r}-0.058 \\
(0.062)\end{array}$ & $\begin{array}{r}-0.056 \\
(0.062)\end{array}$ \\
\hline $\mathrm{ECM}^{*}$ & * stage of business cycle & & & & & & & $\begin{array}{r}0.090 \\
(0.101)\end{array}$ \\
\hline & \# of Observations & 5677 & 5677 & 5677 & 5677 & 3316 & 3250 & 3250 \\
\hline & Adjusted R-squared & 0.297 & 0.318 & 0.329 & 0.332 & 0.321 & 0.289 & 0.289 \\
\hline & Country dummies & yes & yes & yes & yes & yes & yes & yes \\
\hline & Product dummies & yes & yes & yes & yes & yes & yes & yes \\
\hline
\end{tabular}


Appendix Table 10: Pass-through to Local Competitor Prices

\begin{tabular}{|c|c|c|c|c|c|c|c|c|}
\hline change in & exchange rate & $\begin{array}{c}\text { Eqn 1 } \\
0.396 \text { *** } \\
(0.027)\end{array}$ & $\begin{array}{c}\text { Eqn2 } \\
0.162 * * \\
(0.064)\end{array}$ & $\begin{array}{c}\text { Eqn3 } \\
0.467 * * * \\
(0.082)\end{array}$ & $\begin{array}{c}\text { Eqn 4 } \\
0.470 * * * \\
(0.082)\end{array}$ & $\begin{array}{r}\text { Eqn 5 } \\
-0.688 \\
(0.791)\end{array}$ & $\begin{array}{r}\text { Eqn 6 } \\
-0.961 \\
(0.796)\end{array}$ & $\begin{array}{r}\text { Eqn } 7 \\
-0.898 \\
(0.811)\end{array}$ \\
\hline & depreciations & & $\begin{array}{r}-0.085 \\
(0.092)\end{array}$ & $\begin{array}{r}-0.067 \\
(0.093)\end{array}$ & $\begin{array}{r}-0.078 \\
(0.095)\end{array}$ & $\begin{array}{r}-0.064 \\
(0.115)\end{array}$ & $\begin{array}{r}-0.118 \\
(0.118)\end{array}$ & $\begin{array}{r}-0.115 \\
(0.118)\end{array}$ \\
\hline & depreciations above $25 \%$ & & $\begin{array}{l}0.475^{* * * *} \\
(0.052)\end{array}$ & $\begin{array}{l}0.395 * * * \\
(0.054)\end{array}$ & $\begin{array}{l}0.388 \text { *** } \\
(0.054)\end{array}$ & $\begin{array}{l}0.265 * * * \\
(0.090)\end{array}$ & $\begin{array}{l}0.228 \text { ** } \\
(0.089)\end{array}$ & $\begin{array}{l}0.236 * * * \\
(0.088)\end{array}$ \\
\hline change in & exporter's price & $\begin{array}{c}0.052 * \\
(0.027)\end{array}$ & $\begin{array}{r}0.035 \\
(0.027)\end{array}$ & $\begin{array}{r}0.026 \\
(0.027)\end{array}$ & $\begin{array}{r}0.027 \\
(0.027)\end{array}$ & $\begin{array}{r}0.007 \\
(0.037)\end{array}$ & $\begin{array}{r}0.010 \\
(0.035)\end{array}$ & $\begin{array}{r}0.009 \\
(0.035)\end{array}$ \\
\hline$($ delta s)* & trend & & & $\begin{array}{l}-0.042 * * * \\
(0.007)\end{array}$ & $\begin{array}{l}-0.043^{* * *} \\
(0.007)\end{array}$ & $\begin{array}{l}-0.070 * * * \\
(0.010)\end{array}$ & $\begin{array}{l}-0.047^{* * * *} \\
(0.011)^{2}\end{array}$ & $\begin{array}{l}-0.048^{* * *} \\
(0.010)\end{array}$ \\
\hline$($ delta s)* & $\begin{array}{l}\log [\text { per capitaRGDP(importer) / } \\
\text { per capitaRGDP(exporter)] }\end{array}$ & & & & $\begin{array}{r}-0.007 \\
(0.006)\end{array}$ & $\begin{array}{l}-0.070 * * \\
(0.027)\end{array}$ & $\begin{array}{l}-0.092 * * * \\
(0.024)\end{array}$ & $\begin{array}{l}-0.088^{* * *} \\
(0.024)\end{array}$ \\
\hline$($ delta s)* & tariff levels & & & & & $\begin{array}{r}-0.014 \\
(0.134)\end{array}$ & $\begin{array}{r}0.120 \\
(0.135)\end{array}$ & $\begin{array}{r}0.114 \\
(0.135)\end{array}$ \\
\hline$($ delta s)* & $\log$ distance & & & & & $\begin{array}{l}0.176^{* * * *} \\
(0.053)\end{array}$ & $\begin{array}{l}0.130^{* * * *} \\
(0.049)\end{array}$ & $\begin{array}{l}0.126 * * \\
(0.051)\end{array}$ \\
\hline$($ delta s)* & $\log [$ RGDP(importer)/RGDP(expor & & & & & $\begin{array}{l}0.062 * * \\
(0.024)\end{array}$ & $\begin{array}{l}0.080^{* * * *} \\
(0.023)\end{array}$ & $\begin{array}{l}0.076^{* * * *} \\
(0.023)\end{array}$ \\
\hline$($ delta s)* & log real wage $(\$)$ & & & & & $\begin{array}{l}-0.017 * * \\
(0.007)\end{array}$ & $\begin{array}{r}-0.009 \\
(0.006)\end{array}$ & $\begin{array}{r}-0.009 \\
(0.006)\end{array}$ \\
\hline$($ delta s)* & long term inflation & & & & & & $\begin{array}{l}0.587 \text { *** } \\
(0.244)\end{array}$ & $\begin{array}{l}0.594^{* *} \\
(0.235)\end{array}$ \\
\hline$($ delta s)* & long term exchange rate variability & & & & & & $\begin{array}{r}-2.327 \\
(1.635)\end{array}$ & $\begin{array}{r}-2.352 \\
(1.658)\end{array}$ \\
\hline$($ delta s)* & US Importer dummy & & & & & & $\begin{array}{l}0.484^{* * * *} \\
(0.184)\end{array}$ & $\begin{array}{l}0.5011^{* * *} \\
(0.183)^{2}\end{array}$ \\
\hline$($ delta s)* & stage of business cycle & & & & & & & $\begin{array}{r}0.137 \\
(0.696)\end{array}$ \\
\hline & Error Correction term (ECM) & $\begin{array}{l}-0.018 * * * \\
(0.006)\end{array}$ & $\begin{array}{l}-0.014 * * \\
(0.006)\end{array}$ & $\begin{array}{r}0.008 \\
(0.014)\end{array}$ & $\begin{array}{r}0.007 \\
(0.014)\end{array}$ & $\begin{array}{r}-0.011 \\
(0.114)\end{array}$ & $\begin{array}{r}-0.033 \\
(0.111)\end{array}$ & $\begin{array}{r}0.013 \\
(0.117)\end{array}$ \\
\hline $\mathrm{ECM}^{*}$ & trend & & & $\begin{array}{l}-0.003 \text { ** } \\
(0.001)\end{array}$ & $\begin{array}{l}-0.003 \text { ** } \\
(0.001)\end{array}$ & $\begin{array}{r}-0.001 \\
(0.002)\end{array}$ & $\begin{array}{r}-0.002 \\
(0.002)\end{array}$ & $\begin{array}{r}-0.002 \\
(0.002)\end{array}$ \\
\hline $\mathrm{ECM}^{*}$ & $\begin{array}{l}\text { log[per capitaRGDP(importer) / } \\
\text { per capitaRGDP(exporter)] }\end{array}$ & & & & $\begin{array}{r}-0.001 \\
(0.002)\end{array}$ & $\begin{array}{l}-0.020 * * * \\
(0.006)\end{array}$ & $\begin{array}{l}-0.017 \text { **** } \\
(0.006)\end{array}$ & $\begin{array}{l}-0.016 * * * \\
(0.006)\end{array}$ \\
\hline $\mathrm{ECM}^{*}$ & tariff levels & & & & & $\begin{array}{r}-0.028 \\
(0.020)\end{array}$ & $\begin{array}{r}-0.028 \\
(0.020)\end{array}$ & $\begin{array}{r}-0.030 \\
(0.020)\end{array}$ \\
\hline $\mathrm{ECM}^{*}$ & log distance & & & & & $\begin{array}{l}0.027 * * * \\
(0.008)\end{array}$ & $\begin{array}{l}0.027^{* * * *} \\
(0.009)\end{array}$ & $\begin{array}{l}0.023 \text { ** } \\
(0.009)\end{array}$ \\
\hline $\mathrm{ECM}^{*}$ & $\log [$ RGDP(importer)/RGDP(expor & & & & & $\begin{array}{l}0.015 * * * \\
(0.005)\end{array}$ & $\begin{array}{l}0.012 \text { ** } \\
(0.005)\end{array}$ & $\begin{array}{l}0.010 \text { ** } \\
(0.005)\end{array}$ \\
\hline $\mathrm{ECM}^{*}$ & log real wage $(\$)$ & & & & & $\begin{array}{l}0.019 * * * \\
(0.007)\end{array}$ & $\begin{array}{l}0.015 \text { ** } \\
(0.007)\end{array}$ & $\begin{array}{l}0.014 \text { ** } \\
(0.007)\end{array}$ \\
\hline $\mathrm{ECM}^{*}$ & long term inflation & & & & & & $\begin{array}{r}0.012 \\
(0.025)\end{array}$ & $\begin{array}{r}0.019 \\
(0.025)\end{array}$ \\
\hline $\mathrm{ECM}^{*}$ & long term exchange rate variability & & & & & & $\begin{array}{l}-0.158 * * \\
(0.074)\end{array}$ & $\begin{array}{l}-0.146 * \\
(0.077)\end{array}$ \\
\hline $\mathrm{ECM}^{*}$ & US Importer dummy & & & & & & $\begin{array}{r}-0.021 \\
(0.054)\end{array}$ & $\begin{array}{r}-0.007 \\
(0.053)\end{array}$ \\
\hline $\mathrm{ECM}^{*}$ & stage of business cycle & & & & & & & $\begin{array}{r}0.298^{* *} \\
(0.135)^{* *}\end{array}$ \\
\hline & \# of Observations & 5630 & 5630 & 5630 & 5630 & 3299 & 3235 & 3187 \\
\hline & Adjusted R-squared & 0.224 & 0.246 & 0.254 & 0.254 & 0.246 & 0.262 & 0.269 \\
\hline & Country dummies & yes & yes & yes & yes & yes & yes & yes \\
\hline & Product dummies & yes & yes & yes & yes & yes & yes & yes \\
\hline
\end{tabular}


Appendix Table 11: Pass-through to Imported the CPI

\begin{tabular}{|c|c|c|c|c|c|c|c|c|c|c|}
\hline & & Eqn 1 & Eqn2 & Eqn3 & Eqn 4 & Eqn 5 & Eqn 6 & Eqn 7 & Eqn 8 & Eqn 9 \\
\hline change in & exchange rate & $\begin{array}{l}0.279^{* * * *} \\
(0.052)\end{array}$ & $\begin{array}{l}-0.368 \text { *** } \\
(0.095)\end{array}$ & $\begin{array}{r}-0.129 \\
(0.113)\end{array}$ & $\begin{array}{r}-0.035 \\
(0.093)\end{array}$ & $\begin{array}{r}0.777 \\
(1.187)\end{array}$ & $\begin{array}{r}2.162 \\
(1.571)\end{array}$ & $\begin{array}{l}5.069^{* * *} \\
(1.780)\end{array}$ & $\begin{array}{l}2.726 * * \\
(1.241)\end{array}$ & $\begin{array}{r}-0.702 \\
(1.350)\end{array}$ \\
\hline & depreciations & & $\begin{array}{l}0.495 \text { **** } \\
(0.127)\end{array}$ & $\begin{array}{l}0.585 * * * \\
(0.134)\end{array}$ & $\begin{array}{l}0.615^{* * *} \\
(0.113)^{*}\end{array}$ & $\begin{array}{l}0.539^{* * * *} \\
(0.102)^{2}\end{array}$ & $\begin{array}{l}0.548 \text { **** } \\
(0.105)\end{array}$ & $\begin{array}{l}0.407 * * \\
(0.184)\end{array}$ & $\begin{array}{r}0.071 \\
(0.095)\end{array}$ & $\begin{array}{l}0.168 * \\
(0.089)\end{array}$ \\
\hline & depreciations above $25 \%$ & & $\begin{array}{l}0.352 \text { *** } \\
(0.063)^{2}\end{array}$ & $\begin{array}{l}0.243 * * * \\
(0.062)\end{array}$ & $\begin{array}{l}0.295^{* * *} \\
(0.070)\end{array}$ & $\begin{array}{l}0.314^{* * *} \\
(0.075)^{*}\end{array}$ & $\begin{array}{l}0.289 * * * \\
(0.069)\end{array}$ & $\begin{array}{r}0.156 \\
(0.103)\end{array}$ & $\begin{array}{l}0.147^{* * *} \\
(0.052)\end{array}$ & $\begin{array}{l}0.094 * \\
(0.050)\end{array}$ \\
\hline$($ delta s)* & trend & & & $\begin{array}{l}-0.042 * * * \\
(0.009)\end{array}$ & $\begin{array}{l}-0.044^{* * *} \\
(0.009)\end{array}$ & $\begin{array}{l}-0.044^{* * *} \\
(0.010)\end{array}$ & $\begin{array}{l}-0.047^{* * *} \\
(0.010)\end{array}$ & $\begin{array}{l}-0.062 * * * \\
(0.013)\end{array}$ & $\begin{array}{r}-0.007 \\
(0.008)\end{array}$ & $\begin{array}{r}-0.003 \\
(0.008)\end{array}$ \\
\hline (delta s)* & $\begin{array}{l}\log [\text { per capitaRGDP(importer) } / \\
\text { per capitaRGDP(exporter)] }\end{array}$ & & & & $\begin{array}{l}0.078^{* *} \\
(0.032)\end{array}$ & $\begin{array}{l}0.075^{* *} \\
(0.036)\end{array}$ & $\begin{array}{r}0.021 \\
(0.032)\end{array}$ & $\begin{array}{l}0.735^{* * * *} \\
(0.183)^{*}\end{array}$ & $\begin{array}{l}0.4377^{* * *} \\
(0.104)\end{array}$ & $\begin{array}{l}0.452 \text { **** } \\
(0.090)\end{array}$ \\
\hline$($ delta s)* & tariff levels & & & & & $\begin{array}{l}-0.384 * \\
(0.233)\end{array}$ & $\begin{array}{l}-0.669 * * \\
(0.309)\end{array}$ & $\begin{array}{l}-1.120^{* * *} \\
(0.407)\end{array}$ & $\begin{array}{r}-0.408 \\
(0.264)\end{array}$ & $\begin{array}{r}0.317 \\
(0.270)\end{array}$ \\
\hline$($ delta s)* & log distance & & & & & $\begin{array}{l}0.116^{* *} \\
(0.058)\end{array}$ & $\begin{array}{l}0.134 \text { ** } \\
(0.068)\end{array}$ & $\begin{array}{r}-0.019 \\
(0.108)\end{array}$ & $\begin{array}{c}-0.109 * \\
(0.060)\end{array}$ & $\begin{array}{l}-0.110 * \\
(0.057)\end{array}$ \\
\hline$($ delta s)* & $\log [$ RGDP(importer)/RGDP(expo & ter)] & & & & & $\begin{array}{l}0.065 \text { ** } \\
(0.032)\end{array}$ & $\begin{array}{r}0.040 \\
(0.033)\end{array}$ & $\begin{array}{l}0.054^{* * * *} \\
(0.019)\end{array}$ & $\begin{array}{l}0.051 \text { **** } \\
(0.018)\end{array}$ \\
\hline$($ delta s)* & log real wage $(\$)$ & & & & & & & $\begin{array}{l}-0.4555^{* * *} \\
(0.085)\end{array}$ & $\begin{array}{l}-0.2922^{* * *} \\
(0.052)\end{array}$ & $\begin{array}{l}-0.281 \text { *** } \\
(0.046)\end{array}$ \\
\hline$($ delta s)* & long term inflation & & & & & & & & $\begin{array}{l}0.857^{* * * *} \\
(0.124)\end{array}$ & $\begin{array}{l}0.775^{* * *} \\
(0.114)\end{array}$ \\
\hline$($ delta s)* & long term exchange rate variability & & & & & & & & $\begin{array}{l}-4.257 \text { **** } \\
(0.870)\end{array}$ & $\begin{array}{l}-4.784 * * * * \\
(0.770)\end{array}$ \\
\hline$($ delta s)* & stage of business cycle & & & & & & & & & $\begin{array}{l}-1.9999^{* * * *} \\
(0.393)\end{array}$ \\
\hline & Error Correction term (ECM) & $\begin{array}{c}-0.014 \\
(0.009)\end{array}$ & $\begin{array}{r}-0.011 \\
0.007\end{array}$ & $\begin{array}{r}-0.002 \\
0.005\end{array}$ & $\begin{array}{r}-0.001 \\
0.005\end{array}$ & $\begin{array}{r}-0.338 \\
0.243\end{array}$ & $\begin{array}{r}-0.346 \\
(0.232)\end{array}$ & $\begin{array}{c}-0.740 * \\
(0.447)\end{array}$ & $\begin{array}{l}0.472 \text { ** } \\
0.201\end{array}$ & $\begin{array}{l}0.468 \text { *** } \\
0.181\end{array}$ \\
\hline $\mathrm{ECM}^{*}$ & trend & & & $\begin{array}{l}0.003 \text { **** } \\
0.001\end{array}$ & $\begin{array}{l}0.006^{* * *} \\
0.001\end{array}$ & $\begin{array}{l}0.009^{\text {****}} \\
0.001\end{array}$ & $\begin{array}{l}0.009 \text { *** } \\
(0.001)\end{array}$ & $\begin{array}{l}0.0122^{* * *} \\
(0.002)\end{array}$ & $\begin{array}{l}0.006 \text { **** } \\
(0.002)\end{array}$ & $\begin{array}{l}0.007 \text { *** } \\
(0.002)\end{array}$ \\
\hline $\mathrm{ECM}^{*}$ & tariff levels & & & & & $\begin{array}{l}0.072 \\
0.051\end{array}$ & $\begin{array}{r}0.074 \\
(0.049)\end{array}$ & $\begin{array}{c}0.160 * \\
(0.096)\end{array}$ & $\begin{array}{l}-0.101 \text { ** } \\
(0.043)\end{array}$ & $\begin{array}{l}-0.100 \text { *** } \\
(0.039)\end{array}$ \\
\hline $\mathrm{ECM}^{*}$ & log distance & & & & & $\begin{array}{l}-0.0066^{* * *} \\
0.002\end{array}$ & $\begin{array}{r}-0.003 \\
(0.003)\end{array}$ & $\begin{array}{l}-0.0144^{* * *} \\
(0.005)\end{array}$ & $\begin{array}{r}0.000 \\
(0.004)\end{array}$ & $\begin{array}{r}-0.002 \\
(0.004)\end{array}$ \\
\hline $\mathrm{ECM}^{*}$ & $\log [$ RGDP(importer)/RGDP(expo & ter)] & & & & & $\begin{array}{r}0.006 \\
(0.007)\end{array}$ & $\begin{array}{r}0.000 \\
(0.008)\end{array}$ & $\begin{array}{r}0.001 \\
(0.006)\end{array}$ & $\begin{array}{r}0.001 \\
(0.006)\end{array}$ \\
\hline $\mathrm{ECM}^{*}$ & log real wage $(\$)$ & & & & & & & $\begin{array}{r}-0.012 \\
(0.028)\end{array}$ & $\begin{array}{r}0.033 \\
(0.020)\end{array}$ & $\begin{array}{r}0.033 \\
(0.020)\end{array}$ \\
\hline $\mathrm{ECM}^{*}$ & long term inflation & & & & & & & & $\begin{array}{l}-0.172 \text { **** } \\
(0.039)\end{array}$ & $\begin{array}{l}-0.180 \text { *** } \\
(0.040)\end{array}$ \\
\hline $\mathrm{ECM}^{*}$ & long term exchange rate variability & & & & & & & & $\begin{array}{l}0.126^{* *} \\
(0.059)\end{array}$ & $\begin{array}{r}0.049 \\
(0.054)\end{array}$ \\
\hline $\mathrm{ECM}^{*}$ & stage of business cycle & & & & & & & & & $\begin{array}{c}-0.153 * \\
(0.087)\end{array}$ \\
\hline & \# of Observations & 732 & 732 & 710 & 710 & 579 & 579 & 350 & 345 & 343 \\
\hline & $\begin{array}{l}\text { Adjusted R-squared } \\
\text { Country dummies }\end{array}$ & $\begin{array}{r}0.693 \\
\text { yes }\end{array}$ & $\begin{array}{r}0.761 \\
\text { yes }\end{array}$ & $\begin{array}{r}0.785 \\
\text { yes }\end{array}$ & $\begin{array}{r}0.795 \\
\text { yes }\end{array}$ & $\begin{array}{r}0.797 \\
\text { yes }\end{array}$ & $\begin{array}{r}0.805 \\
\text { yes }\end{array}$ & $\begin{array}{r}0.856 \\
\text { yes }\end{array}$ & $\begin{array}{r}0.896 \\
\text { yes }\end{array}$ & $\begin{array}{r}0.903 \\
\text { yes }\end{array}$ \\
\hline
\end{tabular}

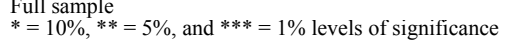


Appendix Table 12: Pass-through to Unit Values

\begin{tabular}{|c|c|c|c|c|c|c|c|c|}
\hline change in & exchange rate & $\begin{array}{c}\text { Eqn 1 } \\
0.666 \text { *** } \\
(0.067)\end{array}$ & $\begin{array}{r}\text { Eqn2 } \\
-0.036 \\
(0.186)\end{array}$ & $\begin{array}{r}\text { Eqn3 } \\
-0.336 \\
(0.235)\end{array}$ & $\begin{array}{r}\text { Eqn 4 } \\
-0.386 \\
(0.237)\end{array}$ & $\begin{array}{r}\text { Eqn 5 } \\
1.446 \\
(1.491)\end{array}$ & $\begin{array}{r}\text { Eqn 6 } \\
1.143 \\
(1.515)\end{array}$ & $\begin{array}{r}\text { Eqn } 7 \\
0.645 \\
(1.500)\end{array}$ \\
\hline & depreciations & & $\begin{array}{l}1.0211^{* * *} \\
(0.274)^{2}\end{array}$ & $\begin{array}{l}1.048^{* * * *} \\
(0.274)^{2}\end{array}$ & $\begin{array}{l}1.130 * * * \\
(0.277)\end{array}$ & $\begin{array}{l}0.609 \text { ** } \\
(0.301)\end{array}$ & $\begin{array}{l}0.625 \text { ** } \\
(0.305)\end{array}$ & $\begin{array}{r}0.441 \\
(0.306)\end{array}$ \\
\hline & depreciations above $25 \%$ & & $\begin{array}{r}-0.160 \\
(0.150)\end{array}$ & $\begin{array}{r}-0.157 \\
(0.152)\end{array}$ & $\begin{array}{r}-0.126 \\
(0.152)\end{array}$ & $\begin{array}{r}-0.195 \\
(0.192)\end{array}$ & $\begin{array}{r}-0.108 \\
(0.209)\end{array}$ & $\begin{array}{r}-0.153 \\
(0.211)\end{array}$ \\
\hline change in & exporter's price & $\begin{array}{r}0.094 \\
(0.063)\end{array}$ & $\begin{array}{r}0.082 \\
(0.063)\end{array}$ & $\begin{array}{l}0.122 * \\
(0.066)\end{array}$ & $\begin{array}{l}0.122 * \\
(0.066)\end{array}$ & $\begin{array}{l}0.263^{* * * *} \\
(0.073)^{2}\end{array}$ & $\begin{array}{l}0.252 * * * \\
(0.074)\end{array}$ & $\begin{array}{l}0.211 \text { *** } \\
(0.074)^{2}\end{array}$ \\
\hline$($ delta s)* & trend & & & $\begin{array}{l}0.042 \text { ** } \\
(0.017)\end{array}$ & $\begin{array}{l}0.046^{* * *} \\
(0.017)\end{array}$ & $\begin{array}{l}0.048^{* *} \\
(0.022)\end{array}$ & $\begin{array}{l}0.040 * \\
(0.024)\end{array}$ & $\begin{array}{l}0.041 * \\
(0.024)\end{array}$ \\
\hline$($ delta s)* & $\begin{array}{l}\log [\text { per capitaRGDP(importer) / } \\
\text { per capitaRGDP(exporter)] }\end{array}$ & & & & $\begin{array}{l}0.043^{* * *} \\
(0.016)\end{array}$ & $\begin{array}{r}0.084 \\
(0.056)\end{array}$ & $\begin{array}{l}0.137 * * \\
(0.058)\end{array}$ & $\begin{array}{l}0.1311^{* *} \\
(0.058)\end{array}$ \\
\hline$($ delta s)* & tariff levels & & & & & $\begin{array}{r}-0.039 \\
(0.292)\end{array}$ & $\begin{array}{r}-0.022 \\
(0.301)\end{array}$ & $\begin{array}{r}0.014 \\
(0.299)\end{array}$ \\
\hline$($ delta s)* & log distance & & & & & $\begin{array}{l}-0.154 * \\
(0.085)\end{array}$ & $\begin{array}{r}-0.132 \\
(0.094)\end{array}$ & $\begin{array}{r}-0.132 \\
(0.095)\end{array}$ \\
\hline$($ delta s)* & $\log [$ RGDP(importer)/RGDP(exporter & & & & & $\begin{array}{l}-0.088 * \\
(0.051)\end{array}$ & $\begin{array}{l}-0.131 * * \\
(0.051)\end{array}$ & $\begin{array}{l}-0.115 \text { ** } \\
(0.051)\end{array}$ \\
\hline$($ delta s)* & log real wage $(\$)$ & & & & & $\begin{array}{r}-0.001 \\
(0.013)\end{array}$ & $\begin{array}{r}-0.019 \\
(0.016)\end{array}$ & $\begin{array}{r}-0.025 \\
(0.017)\end{array}$ \\
\hline$($ delta s)* & long term inflation & & & & & & $\begin{array}{r}-0.625 \\
(0.400)\end{array}$ & $\begin{array}{r}-0.559 \\
(0.400)\end{array}$ \\
\hline$($ delta s)* & long term exchange rate variability & & & & & & $\begin{array}{r}1.545 \\
(2.221)\end{array}$ & $\begin{array}{r}1.391 \\
(2.235)\end{array}$ \\
\hline$($ delta s)* & US Importer dummy & & & & & & $\begin{array}{r}-0.043 \\
(0.274)\end{array}$ & $\begin{array}{r}0.244 \\
(0.268)\end{array}$ \\
\hline$($ delta s)* & stage of business cycle & & & & & & & $\begin{array}{l}0.602 \text { *** } \\
(0.142)\end{array}$ \\
\hline & Error Correction term (ECM) & $\begin{array}{l}-0.218^{* * *} \\
(0.030)\end{array}$ & $\begin{array}{l}-0.221 * * * \\
(0.030)\end{array}$ & $\begin{array}{l}-0.187^{* * * *} \\
(0.033)^{2}\end{array}$ & $\begin{array}{l}-0.1911^{* * *} \\
(0.034)\end{array}$ & $\begin{array}{r}0.236 \\
(0.148)\end{array}$ & $\begin{array}{l}0.352 * * \\
(0.146)\end{array}$ & $\begin{array}{l}0.332 \text { ** } \\
(0.159)\end{array}$ \\
\hline $\mathrm{ECM}^{*}$ & trend & & & $\begin{array}{l}-0.005 * * \\
(0.002)\end{array}$ & $\begin{array}{l}-0.005 * * \\
(0.002)\end{array}$ & $\begin{array}{l}-0.006 * \\
(0.003)\end{array}$ & $\begin{array}{l}-0.007 * * \\
(0.003)\end{array}$ & $\begin{array}{l}-0.006 * * \\
(0.003)\end{array}$ \\
\hline $\mathrm{ECM}^{*}$ & $\begin{array}{l}\log [\text { per capitaRGDP(importer) / } \\
\text { per capitaRGDP(exporter)] }\end{array}$ & & & & $\begin{array}{r}-0.002 \\
(0.003)\end{array}$ & $\begin{array}{r}-0.008 \\
(0.009)\end{array}$ & $\begin{array}{r}-0.003 \\
(0.009)\end{array}$ & $\begin{array}{r}-0.003 \\
(0.009)\end{array}$ \\
\hline $\mathrm{ECM}^{*}$ & tariff levels & & & & & $\begin{array}{r}0.003 \\
(0.024)\end{array}$ & $\begin{array}{r}-0.019 \\
(0.021)\end{array}$ & $\begin{array}{r}-0.013 \\
(0.022)\end{array}$ \\
\hline $\mathrm{ECM}^{*}$ & log distance & & & & & $\begin{array}{l}-0.045 * * * \\
(0.017)\end{array}$ & $\begin{array}{l}-0.049^{* * *} \\
(0.019)\end{array}$ & $\begin{array}{l}-0.050 \text { ** } \\
(0.020)\end{array}$ \\
\hline $\mathrm{ECM}^{*}$ & $\log [$ RGDP(importer)/RGDP(exporter & & & & & $\begin{array}{r}0.007 \\
(0.007)\end{array}$ & $\begin{array}{r}0.004 \\
(0.007)\end{array}$ & $\begin{array}{r}0.003 \\
(0.008)\end{array}$ \\
\hline $\mathrm{ECM}^{*}$ & log real wage (\$) & & & & & $\begin{array}{r}-0.002 \\
(0.015)\end{array}$ & $\begin{array}{r}-0.016 \\
(0.016)\end{array}$ & $\begin{array}{r}-0.014 \\
(0.017)\end{array}$ \\
\hline $\mathrm{ECM}^{*}$ & long term inflation & & & & & & $\begin{array}{r}-0.015 \\
(0.052)\end{array}$ & $\begin{array}{r}-0.006 \\
(0.053)\end{array}$ \\
\hline $\mathrm{ECM}^{*}$ & long term exchange rate variability & & & & & & $\begin{array}{r}-0.156 \\
(0.107)\end{array}$ & $\begin{array}{r}-0.174 \\
(0.114)\end{array}$ \\
\hline $\mathrm{ECM}^{*}$ & US Importer dummy & & & & & & $\begin{array}{r}0.149 \\
(0.110)\end{array}$ & $\begin{array}{r}0.143 \\
(0.109)\end{array}$ \\
\hline $\mathrm{ECM}^{*}$ & stage of business cycle & & & & & & & $\begin{array}{r}0.115 \\
(0.158)\end{array}$ \\
\hline & \# of Observations & 3774 & 3774 & 3774 & 3774 & 2625 & 2561 & 2522 \\
\hline & Adjusted R-squared & 0.151 & 0.155 & 0.157 & 0.158 & 0.154 & 0.155 & 0.161 \\
\hline & Country dummies & yes & yes & yes & yes & yes & yes & yes \\
\hline & Product dummies & yes & yes & yes & yes & yes & yes & yes \\
\hline
\end{tabular}


Appendix Table 13. Determination of Passthrough to Imported Goods Priees: Developing countries, Relative to Rich countries

\begin{tabular}{|c|c|c|c|c|c|c|c|c|c|c|c|c|c|c|c|c|c|}
\hline \multirow{3}{*}{ change in } & \multirow{3}{*}{ exchange rate } & \multirow{2}{*}{\multicolumn{4}{|c|}{ Equation 1 $\quad$ Equation2 }} & \multirow{2}{*}{\multicolumn{2}{|c|}{$\begin{array}{c}\text { Equation } 3 \\
\text { Rich }\end{array}$}} & \multirow{2}{*}{\multicolumn{2}{|c|}{$\begin{array}{c}\text { Equation } 4 \\
\text { Rich }\end{array}$}} & \multirow{2}{*}{\multicolumn{2}{|c|}{ Equation 5}} & \multicolumn{2}{|c|}{ Equation 6} & \multicolumn{2}{|c|}{ Equation 7} & \multicolumn{2}{|c|}{ Equation 8} \\
\hline & & & D Dev. & Rich & D Dev. & & & Rich & & Rich & & Rich & D Dev. & & D Dev. & Rich & D Dev. \\
\hline & & $\begin{array}{l}-0.052 \\
(0.059)\end{array}$ & $\begin{aligned} 0.125 \\
(0.111)\end{aligned}$ & $\begin{aligned} 0.126 \\
(0.092)\end{aligned}$ & $\begin{array}{c}0.249 * \\
(0.150)\end{array}$ & $\begin{array}{l}0.119 * * * \\
(0.003)\end{array}$ & $\begin{array}{l}0.232 \\
(0.153)\end{array}$ & $\begin{array}{l}0.869 * * \\
(0.339)\end{array}$ & $\begin{aligned} 0.257 \\
(0.610)\end{aligned}$ & $\begin{array}{l}0.847 \text { ** } \\
(0.335)\end{array}$ & $\begin{aligned} 0.244 \\
(0.612)\end{aligned}$ & $\begin{array}{c}0.821 \text { ** } \\
(0.331)\end{array}$ & $\begin{aligned} 0.972 \\
(0.825)\end{aligned}$ & $\begin{array}{l}0.984 * * \\
(0.383)\end{array}$ & $\begin{aligned} 0.232 \\
(0.879)\end{aligned}$ & $\begin{array}{r}0.590 \\
(0.372)\end{array}$ & $\begin{array}{r}0.709 \\
(0.887)\end{array}$ \\
\hline & depreciations & $\begin{array}{l}0.267 * * * \\
(0.088)\end{array}$ & $\begin{array}{r}-0.070 \\
(0.157)\end{array}$ & $\begin{array}{l}0.303 * * * \\
(0.089)\end{array}$ & $\begin{array}{r}-0.108 \\
(0.157)\end{array}$ & $\begin{array}{l}0.305 * * * \\
(0.004)\end{array}$ & $\begin{array}{l}-0.137 \\
(0.162)\end{array}$ & $\begin{array}{l}0.275 * * * \\
(0.103)\end{array}$ & $\begin{array}{r}-0.084 \\
(0.173)\end{array}$ & $\begin{array}{l}0.254 * * \\
(0.110)\end{array}$ & $\begin{array}{l}-0.027 \\
(0.181)\end{array}$ & $\begin{array}{l}0.235 * * \\
(0.112)\end{array}$ & $\begin{array}{r}-0.221 \\
(0.269)\end{array}$ & $\begin{array}{l}0.227 \text { ** } \\
(0.110)\end{array}$ & $\begin{array}{r}-0.388 \\
(0.256)\end{array}$ & $\begin{array}{l}0.235 * * \\
(0.111)\end{array}$ & $\begin{array}{r}-0.348 \\
(0.261)\end{array}$ \\
\hline & depreciations above $25 \%$ & $\begin{array}{r}0.093 \\
(0.143)\end{array}$ & $\begin{array}{r}0.242 \\
(0.155)\end{array}$ & $\begin{array}{r}0.064 \\
(0.149)\end{array}$ & $\begin{array}{r}0.197 \\
(0.162)\end{array}$ & $\begin{array}{r}0.078 \\
(0.149)\end{array}$ & $\begin{array}{r}0.172 \\
(0.162)\end{array}$ & $\begin{array}{r}0.105 \\
(0.151)\end{array}$ & $\begin{array}{r}0.125 \\
(0.165)\end{array}$ & $\begin{array}{r}0.116 \\
(0.152)\end{array}$ & $\begin{array}{r}0.108 \\
(0.166)\end{array}$ & $\begin{array}{r}0.128 \\
(0.150)\end{array}$ & $\begin{array}{r}0.000 \\
(0.192)\end{array}$ & $\begin{array}{r}0.238 \\
(0.151)\end{array}$ & $\begin{array}{r}-0.176 \\
(0.192)\end{array}$ & $\begin{array}{r}0.143 \\
(0.149)\end{array}$ & $\begin{array}{r}-0.104 \\
(0.196)\end{array}$ \\
\hline change in & exporter's priee & $\begin{array}{l}0.104 * * * \\
(0.025)\end{array}$ & $\begin{array}{l}-0.058 \\
(0.042)\end{array}$ & $\begin{array}{l}0.105 * * * \\
(0.025)\end{array}$ & $\begin{array}{r}-0.028 \\
(0.043)\end{array}$ & $\begin{array}{l}0.104 * * * \\
(0.025)\end{array}$ & $\begin{array}{r}-0.021 \\
(0.042)\end{array}$ & $\begin{array}{l}0.104 * * * \\
(0.028)\end{array}$ & $\begin{array}{r}-0.023 \\
(0.047)\end{array}$ & $\begin{array}{l}0.104 * * * \\
(0.028)\end{array}$ & $\begin{array}{r}-0.023 \\
(0.047)\end{array}$ & $\begin{array}{l}0.086 * * * \\
(0.029)\end{array}$ & $\begin{array}{r}-0.044 \\
(0.066)\end{array}$ & $\begin{array}{l}0.085 * * * * \\
(0.029)\end{array}$ & $\begin{array}{r}-0.056 \\
(0.065)\end{array}$ & $\begin{array}{l}0.086 * * * \\
(0.029)\end{array}$ & $\begin{array}{r}-0.063 \\
(0.066)\end{array}$ \\
\hline (delta s) $^{*}$ & trend & & & $\begin{array}{l}-0.027 * * * \\
(0.010)\end{array}$ & $\begin{array}{r}-0.013 \\
(0.013)\end{array}$ & $\begin{array}{l}-0.028 * * * \\
(0.010)\end{array}$ & $\begin{array}{r}-0.013 \\
(0.013)\end{array}$ & $\begin{array}{l}-0.026 * * * \\
(0.010)\end{array}$ & $\begin{array}{r}-0.010 \\
(0.013)\end{array}$ & $\begin{array}{l}-0.025 * * \\
(0.010)\end{array}$ & $\begin{array}{l}-0.012 \\
(0.013)\end{array}$ & $\begin{array}{l}-0.022 * * \\
(0.010)\end{array}$ & $\begin{array}{l}-0.060 * * * \\
(0.018)\end{array}$ & $\begin{array}{l}-0.029 * * \\
(0.013)\end{array}$ & $\begin{array}{r}-0.026 \\
(0.021)\end{array}$ & $\begin{array}{r}-0.018 \\
(0.013)\end{array}$ & $\begin{array}{l}-0.039 * \\
(0.021)\end{array}$ \\
\hline$\left(\right.$ delta s) ${ }^{*}$ & $\begin{array}{l}\log [\text { per capitaRGDP(importer) / } \\
\text { per capitaRGDP(exporter)] }\end{array}$ & & & & & $\begin{array}{r}0.007 \\
(0.011)\end{array}$ & $\begin{array}{l}-0.033 * * \\
(0.015)\end{array}$ & $\begin{array}{r}0.004 \\
(0.011)\end{array}$ & $\begin{array}{l}-0.029 * \\
(0.016)\end{array}$ & $\begin{array}{r}0.015 \\
(0.020)\end{array}$ & $\begin{array}{l}-0.061 * * \\
(0.030)\end{array}$ & $\begin{array}{r}0.017 \\
(0.023)\end{array}$ & $\begin{array}{l}-0.080 * \\
(0.044)\end{array}$ & $\begin{array}{r}0.001 \\
(0.026)\end{array}$ & $\begin{array}{r}-0.070 \\
(0.046)\end{array}$ & $\begin{array}{l}-0.024 \\
(0.027)\end{array}$ & $\begin{array}{r}-0.049 \\
(0.046)\end{array}$ \\
\hline$\left(\right.$ delta s)* ${ }^{*}$ & tariff levels & & & & & & & $\begin{array}{r}-0.066 \\
(0.041)\end{array}$ & $\begin{array}{r}-0.012 \\
(0.056)\end{array}$ & $\begin{array}{r}-0.060 \\
(0.041)\end{array}$ & $\begin{array}{l}-0.032 \\
(0.058)\end{array}$ & $\begin{array}{r}-0.051 \\
(0.041)\end{array}$ & $\begin{array}{r}-0.084 \\
(0.080)\end{array}$ & $\begin{array}{r}-0.065 \\
(0.042)\end{array}$ & $\begin{array}{r}0.034 \\
(0.076)\end{array}$ & $\begin{array}{r}-0.055 \\
(0.043)\end{array}$ & $\begin{array}{r}0.028 \\
(0.082)\end{array}$ \\
\hline$(\text { delta })^{*}$ & $\log$ distance & & & & & & & $\begin{array}{l}-0.073 * * \\
(0.036)\end{array}$ & $\begin{array}{r}0.001 \\
(0.063)\end{array}$ & $\begin{array}{l}-0.074 * * \\
(0.036)\end{array}$ & $\begin{array}{r}0.013 \\
(0.065)\end{array}$ & $\begin{array}{l}-0.074 * * \\
(0.036)\end{array}$ & $\begin{array}{r}0.011 \\
(0.092)\end{array}$ & $\begin{array}{r}-0.066 \\
(0.044)\end{array}$ & $\begin{array}{r}0.030 \\
(0.096)\end{array}$ & $\begin{array}{r}-0.018 \\
(0.043)\end{array}$ & $\begin{array}{r}-0.026 \\
(0.097)\end{array}$ \\
\hline (delta s) $^{*}$ & $\log [$ RGDP(importer)/RGDP(exporter)] & & & & & & & & & $\begin{array}{r}-0.011 \\
(0.016)\end{array}$ & $\begin{array}{r}0.034 \\
(0.025)\end{array}$ & $\begin{array}{l}-0.009 \\
(0.020)\end{array}$ & $\begin{array}{r}0.048 \\
(0.042)\end{array}$ & $\begin{array}{r}-0.001 \\
(0.021)\end{array}$ & $\begin{array}{r}0.046 \\
(0.043)\end{array}$ & $\begin{array}{r}0.028 \\
(0.024)\end{array}$ & $\begin{array}{r}0.022 \\
(0.044)\end{array}$ \\
\hline$(\text { delta s) })^{*}$ & $\log$ real wage $(\$)$ & & & & & & & & & & & $\begin{array}{l}-0.019 * * \\
(0.009)\end{array}$ & $\begin{array}{r}0.012 \\
(0.012)\end{array}$ & $\begin{array}{l}-0.021 * * \\
(0.010)\end{array}$ & $\begin{array}{r}0.016 \\
(0.013)\end{array}$ & $\begin{array}{l}-0.023 * * \\
(0.010)\end{array}$ & $\begin{array}{r}0.018 \\
(0.013)\end{array}$ \\
\hline$\left(\right.$ delta s)* ${ }^{*}$ & long term inflation & & & & & & & & & & & & & $\begin{array}{r}-2.337 \\
(2.341)\end{array}$ & $\begin{array}{r}2.760 \\
(2.352)\end{array}$ & $\begin{array}{r}-0.289 \\
(2.354)\end{array}$ & $\begin{array}{r}0.655 \\
(2.367)\end{array}$ \\
\hline$\left(\right.$ delta s) ${ }^{*}$ & long term exchange rate variability & & & & & & & & & & & & & $\begin{array}{r}-3.522 \\
(4.824)\end{array}$ & $\begin{array}{r}1.822 \\
(5.038)\end{array}$ & $\begin{array}{r}-7.818 \\
(4.791)\end{array}$ & $\begin{array}{r}6.144 \\
(5.005)\end{array}$ \\
\hline$\left(\right.$ delta s) ${ }^{*}$ & US Importer dummy & & & & & & & & & & & & & & & $\begin{array}{l}-0.376 * * \\
(0.167)\end{array}$ & \\
\hline$\left(\right.$ delta s) ${ }^{*}$ & stage of business cycle & & & & & & & & & & & & & & & $\begin{array}{l}-2.313 * * * \\
(0.820)\end{array}$ & $\begin{array}{r}1.545 \\
(1.255)\end{array}$ \\
\hline & Error correction term (Ecm) & $\begin{array}{r}-0.093 \\
(0.008)\end{array}$ & $\begin{array}{r}-0.016 \\
(0.010)\end{array}$ & $\begin{array}{l}-0.093 * * * \\
(0.016)\end{array}$ & $\begin{array}{l}-0.101 * * * \\
(0.025)\end{array}$ & $\begin{array}{l}-0.094 * * * \\
(0.016)\end{array}$ & $\begin{array}{l}-0.098 * * * \\
(0.026)\end{array}$ & $\begin{array}{l}-0.302 * * * \\
(0.081)\end{array}$ & $\begin{array}{r}-0.153 \\
(0.152)\end{array}$ & $\begin{array}{l}-0.301 * * * \\
(0.082)\end{array}$ & $\begin{array}{r}-0.161 \\
(0.154)\end{array}$ & $\begin{array}{l}-0.288 * * * \\
(0.080)\end{array}$ & $\begin{array}{l}-0.477 * * \\
(0.222)\end{array}$ & $\begin{array}{l}-0.288 * * * \\
(0.088)\end{array}$ & $\begin{array}{r}-0.110 \\
(0.238)\end{array}$ & $\begin{array}{l}-0.266 * * * \\
(0.089)\end{array}$ & $\begin{array}{r}-0.211 \\
(0.247)\end{array}$ \\
\hline $\mathrm{Ecm} *$ & * trend & & & $\begin{array}{r}0.000 \\
(0.002)\end{array}$ & $\begin{array}{l}0.011 * * * \\
(0.003)\end{array}$ & $\begin{array}{r}0.000 \\
(0.002)\end{array}$ & $\begin{array}{l}0.011 * * * \\
(0.003)\end{array}$ & $\begin{array}{r}0.001 \\
(0.002)\end{array}$ & $\begin{array}{l}0.009 * * * \\
(0.003)\end{array}$ & $\begin{array}{r}0.001 \\
(0.002)\end{array}$ & $\begin{array}{l}0.009 * * * \\
(0.003)\end{array}$ & $\begin{array}{r}0.001 \\
(0.002)\end{array}$ & $\begin{array}{l}0.009 * * \\
(0.004)\end{array}$ & $\begin{array}{r}0.001 \\
(0.002)\end{array}$ & $\begin{array}{r}0.003 \\
(0.005)\end{array}$ & $\begin{array}{r}0.001 \\
(0.002)\end{array}$ & $\begin{array}{r}0.004 \\
(0.005)\end{array}$ \\
\hline & $\begin{array}{c}* \text { *og[per capitaRGDP(importer) } / \\
\text { per capitaRGDP(exporter) }]\end{array}$ & & & & & $\begin{array}{l}-0.004 * \\
(0.002)\end{array}$ & $\begin{array}{r}0.006 \\
(0.004)\end{array}$ & $\begin{array}{r}-0.002 \\
(0.002)\end{array}$ & $\begin{array}{r}0.005 \\
(0.005)\end{array}$ & $\begin{array}{r}0.000 \\
(0.005)\end{array}$ & $\begin{array}{r}0.005 \\
(0.008)\end{array}$ & $\begin{array}{r}0.001 \\
(0.005)\end{array}$ & $\begin{array}{r}0.012 \\
(0.014)\end{array}$ & $\begin{array}{r}0.000 \\
(0.005)\end{array}$ & $\begin{array}{r}0.011 \\
(0.013)\end{array}$ & $\begin{array}{r}0.000 \\
(0.005)\end{array}$ & $\begin{array}{r}0.015 \\
(0.014)\end{array}$ \\
\hline & *" tariff levels & & & & & & & $\begin{array}{r}0.005 \\
(0.008)\end{array}$ & $\begin{array}{r}0.001 \\
(0.011)\end{array}$ & $\begin{array}{r}0.005 \\
(0.008)\end{array}$ & $\begin{array}{r}0.002 \\
(0.012)\end{array}$ & $\begin{array}{r}0.009 \\
(0.009)\end{array}$ & $\begin{array}{r}-0.020 \\
(0.018)\end{array}$ & $\begin{array}{r}0.012 \\
(0.009)\end{array}$ & $\begin{array}{r}-0.028 \\
(0.017)\end{array}$ & $\begin{array}{r}0.005 \\
(0.009)\end{array}$ & $\begin{array}{r}-0.025 \\
(0.018)\end{array}$ \\
\hline & * log distance & & & & & & & $\begin{array}{l}0.023 * * \\
(0.009)\end{array}$ & $\begin{array}{r}0.007 \\
(0.017)\end{array}$ & $\begin{array}{l}0.022 * * \\
(0.010)\end{array}$ & $\begin{array}{r}0.007 \\
(0.017)\end{array}$ & $\begin{array}{l}0.024 * * \\
(0.010)\end{array}$ & $\begin{array}{r}0.039 \\
(0.026)\end{array}$ & $\begin{array}{l}0.023 * * \\
(0.010)\end{array}$ & $\begin{array}{r}0.004 \\
(0.027)\end{array}$ & $\begin{array}{l}0.024 * * \\
(0.010)\end{array}$ & $\begin{array}{r}0.008 \\
(0.028)\end{array}$ \\
\hline & * $\log [$ RGDP(importer)/RGDP(exporter)] & & & & & & & & & $\begin{array}{r}-0.002 \\
(0.004)\end{array}$ & $\begin{array}{r}0.000 \\
(0.007)\end{array}$ & $\begin{array}{r}-0.004 \\
(0.005)\end{array}$ & $\begin{array}{r}-0.005 \\
(0.012)\end{array}$ & $\begin{array}{l}-0.002 \\
(0.005)\end{array}$ & $\begin{array}{c}-0.005 \\
(0.011)\end{array}$ & $\begin{array}{r}-0.002 \\
(0.005)\end{array}$ & $\begin{array}{r}-0.010 \\
(0.011)\end{array}$ \\
\hline & $n^{*} \log$ real wage $(\$)$ & & & & & & & & & & & $\begin{array}{r}0.017 \\
(0.014)\end{array}$ & $\begin{array}{c}-0.027 \\
(0.019)\end{array}$ & $\begin{array}{r}0.014 \\
(0.014)\end{array}$ & $\begin{array}{l}-0.037 \text { ** } \\
(0.019)\end{array}$ & $\begin{array}{r}0.021 \\
(0.015)\end{array}$ & $\begin{array}{l}-0.049 * * \\
(0.021)\end{array}$ \\
\hline & * long term inflation & & & & & & & & & & & & & $\begin{array}{r}0.005 \\
(0.456)\end{array}$ & $\begin{array}{r}-0.092 \\
(0.458)\end{array}$ & $\begin{aligned}-0.070 \\
(0.461)\end{aligned}$ & $\begin{array}{r}-0.001 \\
(0.464)\end{array}$ \\
\hline & * long term exchange rate variability & & & & & & & & & & & & & $\begin{array}{r}0.098 \\
(0.076)\end{array}$ & $\begin{array}{l}-0.336 * * * \\
(0.124)\end{array}$ & $\begin{array}{r}0.104 \\
(0.077)\end{array}$ & $\begin{array}{l}-0.333 * * * \\
(0.128)\end{array}$ \\
\hline & $1^{*}$ US Importer dummy & & & & & & & & & & & & & & & $\begin{array}{c}-0.022 \\
(0.058)\end{array}$ & \\
\hline & $\mathrm{R}^{*}$ stage of business cycle & & & & & & & & & & & & & & & $\begin{array}{c}0.309 * \\
(0.181)\end{array}$ & $\begin{array}{r}0.094 \\
(0.341)\end{array}$ \\
\hline & $\begin{array}{l}\text { \# of Observations } \\
\text { Adjusted R-squared }\end{array}$ & $\begin{array}{c}5677 \\
0.319\end{array}$ & & $\begin{array}{l}5677 \\
0.333\end{array}$ & & $\begin{aligned} 5677 \\
0.33 .\end{aligned}$ & & $\begin{array}{l}4633 \\
0.367\end{array}$ & & $\begin{array}{l}4633 \\
0.367\end{array}$ & & ${ }_{0.348}^{2947}$ & & $\begin{array}{l}2909 \\
0.308\end{array}$ & & $\begin{array}{l}2893 \\
0.313\end{array}$ & \\
\hline
\end{tabular}

$\approx=10 \%, * *=5 \%$, and ${ }^{* * *}=1 \%$ 


\begin{tabular}{|c|c|c|c|c|c|c|c|c|c|c|c|c|c|c|c|c|c|}
\hline \multirow[b]{3}{*}{ change in } & \multirow[b]{3}{*}{ exchange rate } & \multirow{2}{*}{\multicolumn{2}{|c|}{$\begin{array}{c}\text { Equation 1 } \\
\end{array}$}} & \multicolumn{2}{|c|}{$\begin{array}{c}\text { Equation2 } \\
\end{array}$} & \multicolumn{2}{|c|}{$\begin{array}{l}\text { Equation } 3 \\
\end{array}$} & \multicolumn{2}{|c|}{ Equation 4} & \multicolumn{2}{|c|}{ Equation 5} & \multirow{2}{*}{\multicolumn{2}{|c|}{ Equation 6}} & \multicolumn{2}{|c|}{ Equation 7} & \multicolumn{2}{|c|}{$\begin{array}{l}\text { Equation } 8 \\
\end{array}$} \\
\hline & & & & Rich & D Dev. & Rich & D Dev. & Rich & D Dev. & Rich & D Dev. & & & Rich & D Dev. & Rich & D Dev. \\
\hline & & $\begin{array}{r}0.043 \\
(0.061)\end{array}$ & $\begin{array}{l}0.289^{* *} \\
(0.116)\end{array}$ & $\begin{array}{r}0.131 \\
(0.091)\end{array}$ & $\begin{array}{l}0.555 * * * \\
(0.148)\end{array}$ & $\begin{array}{l}0.152 * * * \\
(0.003)\end{array}$ & $\begin{array}{l}0.541 * * * \\
(0.150)\end{array}$ & $\begin{array}{r}-0.074 \\
(0.341)\end{array}$ & $\begin{array}{l}-0.212 \\
(0.594)\end{array}$ & $\begin{array}{c}0.004 \\
(0.344)\end{array}$ & $\begin{array}{l}-0.344 \\
(0.594)\end{array}$ & $\begin{array}{l}-0.037 \\
(0.354)\end{array}$ & $\begin{array}{r}-0.640 \\
(0.917)\end{array}$ & $\begin{array}{r}-0.207 \\
(0.430)\end{array}$ & $\begin{array}{l}-0.142 \\
(0.924)\end{array}$ & $\begin{aligned}-0.323 \\
(0.441)\end{aligned}$ & $\begin{array}{l}-0.109 \\
(0.967)\end{array}$ \\
\hline & depreciations & $\begin{array}{r}-0.071 \\
(0.101)\end{array}$ & $\begin{array}{r}-0.113 \\
(0.168)\end{array}$ & $\begin{array}{r}-0.044 \\
(0.100)\end{array}$ & $\begin{array}{r}-0.150 \\
(0.170)\end{array}$ & $\begin{array}{l}-0.064 * * * \\
(0.003)\end{array}$ & $\begin{array}{r}-0.134 \\
(0.172)\end{array}$ & $\begin{array}{r}-0.024 \\
(0.121)\end{array}$ & $\begin{array}{r}-0.126 \\
(0.194)\end{array}$ & $\begin{array}{r}0.012 \\
(0.127)\end{array}$ & $\begin{array}{r}-0.169 \\
(0.199)\end{array}$ & $\begin{array}{r}0.018 \\
(0.1322\end{array}$ & $\begin{array}{l}-0.442 * \\
(0.268)\end{array}$ & $\begin{array}{l}-0.003 \\
(0.136)\end{array}$ & $\begin{array}{r}-0.383 \\
(0.274)\end{array}$ & $\begin{array}{r}0.058 \\
(0.138)\end{array}$ & $\begin{array}{r}-0.410 \\
(0.278)\end{array}$ \\
\hline & depreciations above $25 \%$ & $\begin{array}{l}0.276 * * \\
(0.127)\end{array}$ & $\begin{array}{r}0.126 \\
(0.141)\end{array}$ & $\begin{array}{l}0.251 * \\
(0.131)\end{array}$ & $\begin{array}{r}0.072 \\
(0.145)\end{array}$ & $\begin{array}{l}0.244 * \\
(0.131)\end{array}$ & $\begin{array}{r}0.078 \\
(0.146)\end{array}$ & $\begin{array}{l}0.246 * \\
(0.135)\end{array}$ & $\begin{array}{r}0.055 \\
(0.151)\end{array}$ & $\begin{array}{l}0.226 * \\
(0.134)\end{array}$ & $\begin{array}{r}0.079 \\
(0.151)\end{array}$ & $\begin{array}{l}0.224 * \\
(0.136)\end{array}$ & $\begin{array}{r}-0.016 \\
(0.179)\end{array}$ & $\begin{array}{r}0.174 \\
(0.149)\end{array}$ & $\begin{array}{r}-0.011 \\
(0.189)\end{array}$ & $\begin{array}{r}0.171 \\
(0.146)\end{array}$ & $\begin{array}{r}-0.016 \\
(0.189)\end{array}$ \\
\hline change in & exporter's priee & $\begin{array}{r}0.029 \\
(0.033)\end{array}$ & $\begin{array}{r}-0.011 \\
(0.050)\end{array}$ & $\begin{array}{r}0.019 \\
(0.033)\end{array}$ & $\begin{array}{r}-0.012 \\
(0.050)\end{array}$ & $\begin{array}{r}0.019 \\
(0.033)\end{array}$ & $\begin{array}{r}-0.011 \\
(0.051)\end{array}$ & $\begin{array}{r}0.017 \\
(0.039)\end{array}$ & $\begin{array}{r}-0.036 \\
(0.057)\end{array}$ & $\begin{array}{r}0.016 \\
(0.039)\end{array}$ & $\begin{array}{r}-0.037 \\
(0.057)\end{array}$ & $\begin{array}{r}0.015 \\
(0.043)\end{array}$ & $\begin{array}{r}-0.075 \\
(0.080)\end{array}$ & $\begin{array}{r}0.013 \\
(0.043)\end{array}$ & $\begin{array}{r}-0.026 \\
(0.077)\end{array}$ & $\begin{array}{r}0.013 \\
(0.043)\end{array}$ & $\begin{array}{r}-0.025 \\
(0.078)\end{array}$ \\
\hline (delta s)* & trend & & & $\begin{array}{r}-0.013 \\
(0.010)\end{array}$ & $\begin{array}{l}-0.034 * * * \\
(0.013)\end{array}$ & $\begin{array}{r}-0.015 \\
(0.010)\end{array}$ & $\begin{array}{l}-0.033 * * * \\
(0.013)\end{array}$ & $\begin{array}{r}-0.013 \\
(0.011)\end{array}$ & $\begin{array}{l}-0.029 * * \\
(0.014)\end{array}$ & $\begin{array}{r}-0.016 \\
(0.011)\end{array}$ & $\begin{array}{l}-0.027 * \\
(0.014)\end{array}$ & $\begin{array}{r}-0.014 \\
(0.011)\end{array}$ & $\begin{array}{l}-0.060 * * * \\
(0.019)\end{array}$ & $\begin{array}{l}-0.007 \\
(0.014)\end{array}$ & $\begin{array}{r}-0.038 \\
(0.023)\end{array}$ & $\begin{array}{r}-0.008 \\
(0.015)\end{array}$ & $\begin{array}{l}-0.040 * \\
(0.023)\end{array}$ \\
\hline (delta s)* & $\begin{array}{l}\log [\text { per capitaRGDP(importer) / } \\
\text { per capitaRGDP(exporter)] }\end{array}$ & & & & & $\begin{array}{r}-0.011 \\
(0.009)\end{array}$ & $\begin{array}{r}0.010 \\
(0.012)\end{array}$ & $\begin{array}{r}-0.006 \\
(0.009)\end{array}$ & $\begin{array}{r}0.003 \\
(0.012)\end{array}$ & $\begin{array}{l}-0.032 * \\
(0.018)\end{array}$ & $\begin{array}{r}0.030 \\
(0.027)\end{array}$ & $\begin{array}{l}-0.039 * \\
(0.021)\end{array}$ & $\begin{array}{r}0.059 \\
(0.047)\end{array}$ & $\begin{array}{l}-0.044 * \\
(0.026)\end{array}$ & $\begin{array}{r}-0.046 \\
(0.044)\end{array}$ & $\begin{array}{r}-0.032 \\
(0.027)\end{array}$ & $\begin{array}{r}-0.057 \\
(0.045)\end{array}$ \\
\hline (delta s)* & tariff levels & & & & & & & $\begin{array}{l}0.079 * \\
(0.040)\end{array}$ & $\begin{array}{r}0.013 \\
(0.059)\end{array}$ & $\begin{array}{r}0.063 \\
(0.042)\end{array}$ & $\begin{array}{r}0.029 \\
(0.061)\end{array}$ & $\begin{array}{r}0.063 \\
(0.042)\end{array}$ & $\begin{array}{r}0.134 \\
(0.084)\end{array}$ & $\begin{array}{r}0.053 \\
(0.045)\end{array}$ & $\begin{array}{r}0.087 \\
(0.090)\end{array}$ & $\begin{array}{l}0.091 * * \\
(0.045)\end{array}$ & $\begin{array}{r}0.063 \\
(0.096)\end{array}$ \\
\hline (delta s) $^{*}$ & log distance & & & & & & & $\begin{array}{r}0.005 \\
(0.040)\end{array}$ & $\begin{array}{r}0.074 \\
(0.065)\end{array}$ & $\begin{array}{r}0.006 \\
(0.041)\end{array}$ & $\begin{array}{r}0.079 \\
(0.065)\end{array}$ & $\begin{array}{r}0.011 \\
(0.042)\end{array}$ & $\begin{array}{r}0.139 \\
(0.101)\end{array}$ & $\begin{array}{r}0.023 \\
(0.054)\end{array}$ & $\begin{array}{r}0.096 \\
(0.102)\end{array}$ & $\begin{array}{r}0.020 \\
(0.056)\end{array}$ & $\begin{array}{r}0.106 \\
(0.106)\end{array}$ \\
\hline (delta s)* & $\log [$ RGDP(importer)/RGDP(exporter)] & & & & & & & & & $\begin{array}{l}0.026 * \\
(0.016)\end{array}$ & $\begin{array}{r}-0.028 \\
(0.024)\end{array}$ & $\begin{array}{l}0.036 * \\
(0.020)\end{array}$ & $\begin{array}{r}-0.069 \\
(0.044)\end{array}$ & $\begin{array}{l}0.040 * \\
(0.022)\end{array}$ & $\begin{array}{r}0.033 \\
(0.042)\end{array}$ & $\begin{array}{r}0.019 \\
(0.024)\end{array}$ & $\begin{array}{r}0.053 \\
(0.043)\end{array}$ \\
\hline (delta s)* & log real wage (\$) & & & & & & & & & & & $\begin{array}{r}-0.005 \\
(0.008)\end{array}$ & $\begin{array}{r}-0.007 \\
(0.011)\end{array}$ & $\begin{array}{r}-0.006 \\
(0.008)\end{array}$ & $\begin{array}{r}0.001 \\
(0.011)\end{array}$ & $\begin{array}{r}-0.004 \\
(0.008)\end{array}$ & $\begin{array}{r}-0.001 \\
(0.011)\end{array}$ \\
\hline (delta s)* & long term inflation & & & & & & & & & & & & & $\begin{array}{r}2.000 \\
(2.997)\end{array}$ & $\begin{array}{r}-1.381 \\
(3.010)\end{array}$ & $\begin{array}{r}1.229 \\
(3.080)\end{array}$ & $\begin{array}{r}-0.642 \\
(3.090)\end{array}$ \\
\hline (delta s)* & long term exchange rate variability & & & & & & & & & & & & & $\begin{array}{r}-0.681 \\
(5.056)\end{array}$ & $\begin{array}{r}-1.953 \\
(5.382)\end{array}$ & $\begin{array}{r}-0.589 \\
(5.193)\end{array}$ & $\begin{array}{r}-2.109 \\
(5.519)\end{array}$ \\
\hline (delta s)* & US Importer dummy & & & & & & & & & & & & & & & $\begin{array}{l}0.824 * * * \\
(0.200)\end{array}$ & \\
\hline (delta s)* & stage of business cycle & & & & & & & & & & & & & & & $\begin{array}{r}-0.832 \\
(0.801)\end{array}$ & $\begin{array}{r}0.371 \\
(1.336)\end{array}$ \\
\hline & Error correction term (Ecm) & $\begin{array}{l}-0.003 \\
(0.008)\end{array}$ & $\begin{array}{r}-0.014 \\
(0.010)\end{array}$ & $\begin{array}{l}0.035 * * \\
(0.016)\end{array}$ & $\begin{array}{r}-0.036 \\
(0.024)\end{array}$ & $\begin{array}{l}0.034 * * \\
(0.016)\end{array}$ & $\begin{array}{r}-0.037 \\
(0.024)\end{array}$ & $\begin{array}{r}-0.039 \\
(0.076)\end{array}$ & $\begin{array}{r}-0.027 \\
(0.137)\end{array}$ & $\begin{array}{r}-0.045 \\
(0.076)\end{array}$ & $\begin{array}{r}0.024 \\
(0.137)\end{array}$ & $\begin{array}{r}-0.035 \\
(0.082)\end{array}$ & $\begin{array}{r}-0.217 \\
(0.207)\end{array}$ & $\begin{array}{r}-0.076 \\
(0.099)\end{array}$ & $\begin{array}{r}-0.225 \\
(0.223)\end{array}$ & $\begin{array}{r}-0.023 \\
(0.102)\end{array}$ & $\begin{array}{r}-0.302 \\
(0.231)\end{array}$ \\
\hline $\mathrm{Ecm}^{*}$ & t trend & & & $\begin{array}{l}-0.005 * * * \\
(0.002)\end{array}$ & $\begin{array}{r}0.003 \\
(0.003)\end{array}$ & $\begin{array}{l}-0.005 * * * \\
(0.002)\end{array}$ & $\begin{array}{r}0.003 \\
(0.003)\end{array}$ & $\begin{array}{r}-0.003 \\
(0.002)\end{array}$ & $\begin{array}{r}0.002 \\
(0.003)\end{array}$ & $\begin{array}{r}-0.003 \\
(0.002)\end{array}$ & $\begin{array}{r}0.001 \\
(0.003)\end{array}$ & $\begin{array}{r}-0.003 \\
(0.002)\end{array}$ & $\begin{array}{r}0.003 \\
(0.004)\end{array}$ & $\begin{array}{r}-0.001 \\
(0.003)\end{array}$ & $\begin{array}{r}0.001 \\
(0.005)\end{array}$ & $\begin{array}{r}-0.002 \\
(0.003)\end{array}$ & $\begin{array}{r}0.002 \\
(0.006)\end{array}$ \\
\hline & $\begin{array}{l}\text { "log[per capitaRGDP(importer)/ } \\
\text { per capitaRGDP(exporter)] }\end{array}$ & & & & & $\begin{array}{l}-0.005 * * \\
(0.002)\end{array}$ & $\begin{array}{r}0.004 \\
(0.003)\end{array}$ & $\begin{array}{l}-0.006 * * \\
(0.002)\end{array}$ & $\begin{array}{r}0.002 \\
(0.003)\end{array}$ & $\begin{array}{l}-0.016 * * * \\
(0.006)\end{array}$ & $\begin{array}{r}0.000 \\
(0.008)\end{array}$ & $\begin{array}{l}-0.015 * * * \\
(0.006)\end{array}$ & $\begin{array}{r}-0.008 \\
(0.013)\end{array}$ & $\begin{array}{l}-0.014 * * * \\
(0.006)\end{array}$ & $\begin{array}{r}-0.003 \\
(0.012)\end{array}$ & $\begin{array}{l}-0.012 * * \\
(0.006)\end{array}$ & $\begin{array}{r}-0.004 \\
(0.013)\end{array}$ \\
\hline $\mathrm{Ecm}^{*}$ & tariff levels & & & & & & & $\begin{array}{r}-0.003 \\
(0.010)\end{array}$ & $\begin{array}{r}-0.001 \\
(0.013)\end{array}$ & $\begin{array}{r}-0.005 \\
(0.010)\end{array}$ & $\begin{array}{r}-0.006 \\
(0.013)\end{array}$ & $\begin{array}{r}-0.004 \\
(0.011)\end{array}$ & $\begin{array}{r}-0.010 \\
(0.019)\end{array}$ & $\begin{array}{c}-0.002 \\
(0.011)\end{array}$ & $\begin{array}{r}-0.018 \\
(0.018)\end{array}$ & $\begin{array}{r}-0.009 \\
(0.011)\end{array}$ & $\begin{array}{r}-0.009 \\
(0.018)\end{array}$ \\
\hline $\mathrm{Ecm}^{*}$ & " log distance & & & & & & & $\begin{array}{r}0.007 \\
(0.009)\end{array}$ & $\begin{array}{r}-0.001 \\
(0.015)\end{array}$ & $\begin{array}{r}0.011 \\
(0.009)\end{array}$ & $\begin{array}{r}-0.005 \\
(0.015)\end{array}$ & $\begin{array}{r}0.009 \\
(0.010)\end{array}$ & $\begin{array}{r}0.037 \\
(0.023)\end{array}$ & $\begin{array}{r}0.011 \\
(0.011)\end{array}$ & $\begin{array}{r}0.035 \\
(0.024)\end{array}$ & $\begin{array}{r}0.009 \\
(0.011)\end{array}$ & $\begin{array}{r}0.039 \\
(0.025)\end{array}$ \\
\hline Ecm* & " $\log [$ RGDP(importer)/RGDP(exporter)] & & & & & & & & & $\begin{array}{l}0.010 \text { ** } \\
(0.005)\end{array}$ & $\begin{array}{r}0.002 \\
(0.007)\end{array}$ & $\begin{array}{r}0.007 \\
(0.005)\end{array}$ & $\begin{array}{r}0.010 \\
(0.010)\end{array}$ & $\begin{array}{c}0.008 * \text { * } \\
(0.005)\end{array}$ & $\begin{array}{c}0.004 \\
(0.010)\end{array}$ & $\begin{array}{r}0.006 \\
(0.005)\end{array}$ & $\begin{array}{c}0.004 \\
(0.010)\end{array}$ \\
\hline $\mathrm{Ecm}^{*}$ & " log real wage (\$) & & & & & & & & & & & $\begin{array}{r}-0.003 \\
(0.016)\end{array}$ & $\begin{array}{r}0.031 \\
(0.020)\end{array}$ & $\begin{array}{r}0.002 \\
(0.015)\end{array}$ & $\begin{array}{r}0.013 \\
(0.019)\end{array}$ & $\begin{array}{r}0.009 \\
(0.015)\end{array}$ & $\begin{array}{r}0.004 \\
(0.019)\end{array}$ \\
\hline $\mathrm{Ecm}^{*}$ & * long term inflation & & & & & & & & & & & & & $\begin{array}{r}0.557 \\
(0.674)\end{array}$ & $\begin{array}{r}-0.531 \\
(0.675)\end{array}$ & $\begin{array}{r}0.473 \\
(0.686)\end{array}$ & $\begin{array}{r}-0.441 \\
(0.687)\end{array}$ \\
\hline $\mathrm{Ecm}^{*}$ & "long term exchange rate variability & & & & & & & & & & & & & $\begin{array}{r}0.043 \\
(0.072)\end{array}$ & $\begin{array}{l}-0.297 \text { *** } \\
(0.136)\end{array}$ & $\begin{array}{r}0.043 \\
(0.073)\end{array}$ & $\begin{array}{l}-0.301 * * \\
(0.141)\end{array}$ \\
\hline Ecm* & " US Importer dummy & & & & & & & & & & & & & & & $\begin{array}{r}0.026 \\
(0.058)\end{array}$ & \\
\hline $\mathrm{Ecm}^{*}$ & "stage of business cycle & & & & & & & & & & & & & & & $\begin{array}{l}0.425 \text { ** } \\
(0.189)\end{array}$ & $\begin{array}{r}-0.264 \\
(0.298)\end{array}$ \\
\hline & $\begin{array}{l}\text { \# of Observations } \\
\text { Adiusted R-saured }\end{array}$ & $\begin{array}{l}5676 \\
0253\end{array}$ & & $\begin{array}{l}5676 \\
0.262\end{array}$ & & $\begin{array}{l}5676 \\
0.262\end{array}$ & & $\begin{array}{l}4591 \\
0.282\end{array}$ & & $\begin{array}{l}4591 \\
0.283\end{array}$ & & $\begin{array}{l}2947 \\
0.257\end{array}$ & & $\begin{array}{l}2909 \\
0.254\end{array}$ & & $\begin{array}{l}2893 \\
0.256\end{array}$ & \\
\hline
\end{tabular}




\begin{tabular}{|c|c|c|c|c|c|c|c|c|c|c|c|c|c|c|c|c|c|}
\hline \multirow[b]{3}{*}{ change in } & \multirow[b]{3}{*}{ exchange rate } & \multirow{2}{*}{\multicolumn{2}{|c|}{$\begin{array}{c}\text { Equation } 1 \\
\end{array}$}} & \multirow{2}{*}{\multicolumn{2}{|c|}{$\begin{array}{c}\text { Equation2 } \\
\text { Pan }\end{array}$}} & \multirow{2}{*}{\multicolumn{2}{|c|}{ Equation 3}} & \multirow{2}{*}{\multicolumn{2}{|c|}{ Equation 4}} & \multicolumn{2}{|c|}{ Equation 5} & \multirow{2}{*}{\multicolumn{2}{|c|}{ Equation 6}} & \multirow{2}{*}{\multicolumn{2}{|c|}{ Equation 7}} & \multicolumn{2}{|c|}{ Equation 8} \\
\hline & & & & & & & & & D Dev. & Rich & D Dev. & & & & & Rich & D Dev. \\
\hline & & $\begin{array}{l}-0.033 \\
(0.033)\end{array}$ & $\begin{array}{l}-0.516{ }^{* * * *} \\
(0.125)\end{array}$ & \begin{tabular}{|c|}
0.008 \\
$(0.031)$
\end{tabular} & $\begin{array}{l}0.283{ }^{*} \\
(0.171)\end{array}$ & $\begin{array}{l}0.002 * \\
(0.001)\end{array}$ & $\begin{array}{r}0.262 \\
(0.182)\end{array}$ & $\begin{array}{r}0.158 \\
(0.209)\end{array}$ & $\begin{array}{l}-0.987 \\
(0.821)\end{array}$ & $\begin{array}{r}0.070 \\
(0.208)\end{array}$ & $\begin{array}{l}-0.764 \\
(0.809)\end{array}$ & $\begin{array}{r}0.116 \\
(0.180)\end{array}$ & $\begin{array}{l}-5.787 * * * \\
(1.935)\end{array}$ & $\begin{array}{c}0.056 \\
(0.185)\end{array}$ & $\begin{array}{l}-3.299 * \\
(1.956)\end{array}$ & $\begin{array}{r}0.015 \\
(0.173)\end{array}$ & $\begin{array}{l}-1.292 \\
(1.675)\end{array}$ \\
\hline & depreciations & $\begin{array}{r}0.058 \\
(0.051)\end{array}$ & $\begin{array}{l}0.638 * * * \\
(0.167)\end{array}$ & $\begin{array}{r}-0.023 \\
(0.059)\end{array}$ & $\begin{array}{l}0.443 * * * \\
(0.141)\end{array}$ & $\begin{array}{l}-0.026 * * * \\
(0.007)\end{array}$ & $\begin{array}{l}0.425 * * * \\
(0.140)\end{array}$ & $\begin{array}{l}-0.014 \\
(0.061)\end{array}$ & $\begin{array}{l}0.536 * * * \\
(0.151)\end{array}$ & $\begin{array}{l}-0.026 \\
(0.065)\end{array}$ & $\begin{array}{l}0.485 * * * \\
(0.148)\end{array}$ & $\begin{array}{l}-0.036 \\
(0.065)\end{array}$ & $\begin{array}{l}0.791 * * * \\
(0.240)\end{array}$ & $\begin{array}{l}-0.038 \\
(0.072)\end{array}$ & $\begin{array}{r}0.305 \\
(0.230)\end{array}$ & $\begin{array}{l}-0.002 \\
(0.061)\end{array}$ & $\begin{array}{r}0.281 \\
(0.186)\end{array}$ \\
\hline & depreciations above $25 \%$ & $\begin{array}{l}0.072 * * * \\
(0.020)\end{array}$ & $\begin{array}{l}0.278 * * * \\
(0.073)\end{array}$ & $\begin{array}{l}0.078 * * * \\
(0.024)\end{array}$ & $\begin{array}{l}0.175 * * \\
(0.087)\end{array}$ & $\begin{array}{l}0.066 * \\
(0.034)\end{array}$ & $\begin{array}{l}0.185 \text { ** } \\
(0.090)\end{array}$ & $\begin{array}{l}0.078 \text { ** } \\
(0.034)\end{array}$ & $\begin{array}{l}0.177 * \\
(0.096)\end{array}$ & $\begin{array}{l}0.080 \text { ** } \\
(0.035)\end{array}$ & $\begin{array}{l}0.179 * \\
(0.095)\end{array}$ & $\begin{array}{r}0.057 \\
(0.035)\end{array}$ & $\begin{array}{r}-0.022 \\
(0.175)\end{array}$ & $\begin{array}{r}0.029 \\
(0.036)\end{array}$ & $\begin{array}{r}0.005 \\
(0.135)\end{array}$ & $\begin{array}{r}0.042 \\
(0.029)\end{array}$ & $\begin{array}{r}-0.062 \\
(0.114)\end{array}$ \\
\hline change in & exporter's priee & $\begin{array}{l}-0.021 * * * * \\
(0.007)\end{array}$ & $\begin{array}{l}-0.076 * * * \\
(0.024)\end{array}$ & $\begin{array}{c}0.011 * \\
(0.006)\end{array}$ & $\begin{array}{l}-0.037 \\
(0.029)\end{array}$ & $\begin{array}{c}0.012 * \\
(0.006)\end{array}$ & $\begin{array}{r}-0.029 \\
(0.029)\end{array}$ & $\begin{array}{r}0.005 \\
(0.007)\end{array}$ & $\begin{array}{c}-0.005 \\
(0.033)\end{array}$ & $\begin{array}{r}0.005 \\
(0.007)\end{array}$ & $\begin{array}{l}-0.019 \\
(0.035)\end{array}$ & $\begin{array}{r}0.003 \\
(0.006)\end{array}$ & $\begin{array}{r}0.044 \\
(0.046)\end{array}$ & $\begin{array}{r}0.002 \\
(0.006)\end{array}$ & $\begin{array}{l}-0.072 * \\
(0.039)\end{array}$ & $\begin{array}{r}0.003 \\
(0.006)\end{array}$ & $\begin{array}{l}-0.087 * * \\
(0.039)\end{array}$ \\
\hline$(\text { delta s) })^{*}$ & trend & & & $\begin{array}{r}0.005 \\
(0.004)\end{array}$ & $\begin{array}{l}-0.080 * * * \\
(0.018)\end{array}$ & $\begin{array}{r}0.004 \\
(0.004)\end{array}$ & $\begin{array}{l}-0.076 * * * \\
(0.020)\end{array}$ & $\begin{array}{r}0.003 \\
(0.005)\end{array}$ & $\begin{array}{l}-0.074 * * * \\
(0.020)\end{array}$ & $\begin{array}{r}0.003 \\
(0.005)\end{array}$ & $\begin{array}{l}-0.071 * * * \\
(0.020)\end{array}$ & $\begin{array}{r}0.004 \\
(0.005)\end{array}$ & $\begin{array}{l}-0.114 * * * \\
(0.027)\end{array}$ & $\begin{array}{c}0.007 \\
(0.008)\end{array}$ & $\begin{array}{l}-0.036 * \\
(0.020)\end{array}$ & $\begin{array}{r}0.002 \\
(0.007)\end{array}$ & $\begin{array}{r}-0.027 \\
(0.020)\end{array}$ \\
\hline$(\text { delta s) })^{*}$ & $\begin{array}{l}\log [\text { per capitaRGDP(importer) / } \\
\text { per capitaRGDP(exporter)] }\end{array}$ & & & & & $\begin{array}{r}-0.047 \\
(0.068)\end{array}$ & $\begin{array}{r}0.032 \\
(0.085)\end{array}$ & $\begin{array}{r}-0.024 \\
(0.072)\end{array}$ & $\begin{array}{r}0.008 \\
(0.097)\end{array}$ & $\begin{array}{r}-0.030 \\
(0.071)\end{array}$ & $\begin{array}{r}0.021 \\
(0.096)\end{array}$ & $\begin{array}{r}-0.091 \\
(0.086)\end{array}$ & $\begin{array}{l}0.900 * * * \\
(0.280)\end{array}$ & $\begin{array}{r}-0.094 \\
(0.102)\end{array}$ & $\begin{array}{l}0.782 * * * \\
(0.198)\end{array}$ & $\begin{array}{r}-0.106 \\
(0.136)\end{array}$ & $\begin{array}{l}0.685 * * * \\
(0.228)\end{array}$ \\
\hline$(\text { delta s) })^{*}$ & tariff levels & & & & & & & $\begin{array}{r}-0.010 \\
(0.013)\end{array}$ & $\begin{array}{r}0.053 \\
(0.072)\end{array}$ & $\begin{array}{r}-0.008 \\
(0.014)\end{array}$ & $\begin{array}{r}0.074 \\
(0.072)\end{array}$ & $\begin{array}{r}0.001 \\
(0.013)\end{array}$ & $\begin{array}{r}-0.047 \\
(0.077)\end{array}$ & $\begin{array}{r}0.010 \\
(0.014)\end{array}$ & $\begin{array}{r}0.063 \\
(0.062)\end{array}$ & $\begin{array}{r}0.007 \\
(0.014)\end{array}$ & $\begin{array}{l}0.151 * \\
(0.078)\end{array}$ \\
\hline$(\text { delta s) })^{*}$ & $\log$ distance & & & & & & & $\begin{array}{r}-0.015 \\
(0.025)\end{array}$ & $\begin{array}{r}0.112 \\
(0.089)\end{array}$ & $\begin{array}{r}-0.009 \\
(0.023)\end{array}$ & $\begin{array}{r}0.073 \\
(0.092)\end{array}$ & $\begin{array}{r}-0.016 \\
(0.020)\end{array}$ & $\begin{array}{l}0.494 * * \\
(0.211)\end{array}$ & $\begin{array}{r}-0.011 \\
(0.017)\end{array}$ & $\begin{array}{l}0.343 * \\
(0.208)\end{array}$ & $\begin{array}{r}-0.014 \\
(0.016)\end{array}$ & $\begin{array}{r}0.223 \\
(0.194)\end{array}$ \\
\hline$(\text { delta s) })^{*}$ & $\log [R G D P($ importer)/RGDP(exporter) $]$ & & & & & & & & & $\begin{array}{r}-0.010 \\
(0.008)\end{array}$ & $\begin{array}{r}-0.027 \\
(0.036)\end{array}$ & $\begin{array}{r}-0.011 \\
(0.008)\end{array}$ & $\begin{array}{l}-0.198 * * \\
(0.084)\end{array}$ & $\begin{array}{l}-0.011 * \\
(0.007)\end{array}$ & $\begin{array}{r}-0.012 \\
(0.068)\end{array}$ & $\begin{array}{l}-0.016 * * \\
(0.007)\end{array}$ & $\begin{array}{l}0.122 * \\
(0.069)\end{array}$ \\
\hline$(\text { delta s) })^{*}$ & $\log$ real wage $(\$)$ & & & & & & & & & & & $\begin{array}{r}0.021 \\
(0.028)\end{array}$ & $\begin{array}{l}-0.547 * * * \\
(0.122)\end{array}$ & $\begin{array}{r}0.029 \\
(0.030)\end{array}$ & $\begin{array}{l}-0.376 * * * \\
(0.083)\end{array}$ & $\begin{array}{r}0.029 \\
(0.038)\end{array}$ & $\begin{array}{l}-0.233 * * * \\
(0.085)\end{array}$ \\
\hline$\left(\right.$ delta s ) ${ }^{*}$ & long term inflation & & & & & & & & & & & & & $\begin{array}{r}0.580 \\
(1.044)\end{array}$ & $\begin{array}{r}-0.279 \\
(1.069)\end{array}$ & $\begin{array}{r}0.270 \\
(0.947)\end{array}$ & $\begin{array}{r}0.065 \\
(0.967)\end{array}$ \\
\hline$(\text { delta s) })^{*}$ & long term exchange rate variability & & & & & & & & & & & & & $\begin{array}{r}0.544 \\
(0.870)\end{array}$ & $\begin{array}{r}-0.541 \\
(1.281)\end{array}$ & $\begin{array}{l}2.143 * * \\
(0.933)\end{array}$ & $\begin{array}{l}-4.427 * * * \\
(1.339)\end{array}$ \\
\hline$\left(\right.$ delta s)* ${ }^{*}$ & stage of business cycle & & & & & & & & & & & & & & & $\begin{array}{r}0.586 \\
(0.409)\end{array}$ & $\begin{array}{l}-3.936 * * * \\
(1.004)\end{array}$ \\
\hline & Error correction term (Ecm) & $\begin{array}{l}-0.037 * * \\
(0.011)\end{array}$ & $\begin{array}{l}0.028 * * \\
(0.013)\end{array}$ & $\begin{array}{l}-0.029 * * * \\
(0.010)\end{array}$ & $\begin{array}{l}0.035 * * * \\
(0.012)\end{array}$ & $\begin{array}{l}-0.029 * * * \\
(0.010)\end{array}$ & $\begin{array}{l}0.036 * * * \\
(0.012)\end{array}$ & $\begin{array}{l}-0.031 * * * \\
(0.011)\end{array}$ & $\begin{array}{l}0.038 * * * \\
(0.013)\end{array}$ & $\begin{array}{l}-0.031 * * * \\
(0.011)\end{array}$ & $\begin{array}{l}0.039 * * * \\
(0.013)\end{array}$ & $\begin{array}{r}-0.013 \\
(0.011)\end{array}$ & $\begin{array}{l}0.031 \text { ** } \\
(0.016)\end{array}$ & $\begin{array}{r}-0.009 \\
(0.010)\end{array}$ & $\begin{array}{r}-0.011 \\
(0.014)\end{array}$ & $\begin{array}{r}-0.007 \\
(0.010)\end{array}$ & $\begin{array}{l}-0.010 \\
(0.013)\end{array}$ \\
\hline Ecm & * trend & & & $\begin{array}{l}-0.001 * * * \\
(0.000)\end{array}$ & $\begin{array}{r}0.000 \\
(0.001)\end{array}$ & $\begin{array}{l}-0.001 * * * \\
(0.000)\end{array}$ & $\begin{array}{l}-0.002 * \\
(0.001)\end{array}$ & $\begin{array}{l}-0.001 * * \\
(0.000)\end{array}$ & $\begin{array}{l}-0.003 * * * \\
(0.001)\end{array}$ & $\begin{array}{l}-0.001 * * \\
(0.000)\end{array}$ & $\begin{array}{l}-0.003 * * \\
(0.001)\end{array}$ & $\begin{array}{l}-0.001 * * * \\
(0.000)\end{array}$ & $\begin{array}{l}-0.004 * * \\
(0.002)\end{array}$ & $\begin{array}{r}0.000 \\
(0.001)\end{array}$ & $\begin{array}{r}0.000 \\
(0.002)\end{array}$ & $\begin{array}{r}0.000 \\
(0.001)\end{array}$ & $\begin{array}{r}0.000 \\
(0.002)\end{array}$ \\
\hline & $\begin{array}{l}\text { * } \log [\text { per capitaRGDP(importer) } / \\
\text { per capitaRGDP(exporter)] }\end{array}$ & & & & & $\begin{array}{r}0.002 \\
(0.005)\end{array}$ & $\begin{array}{r}-0.010 \\
(0.007)\end{array}$ & $\begin{array}{r}0.002 \\
(0.006)\end{array}$ & $\begin{array}{r}0.005 \\
(0.009)\end{array}$ & $\begin{array}{r}0.000 \\
(0.007)\end{array}$ & $\begin{array}{r}0.004 \\
(0.009)\end{array}$ & $\begin{array}{l}0.021 * * \\
(0.008)\end{array}$ & $\begin{array}{l}0.100 * * \\
(0.051)\end{array}$ & $\begin{array}{l}0.014 * \\
(0.008)\end{array}$ & $\begin{array}{r}0.029 \\
(0.042)\end{array}$ & $\begin{array}{r}0.006 \\
(0.009)\end{array}$ & $\begin{array}{r}0.057 \\
(0.037)\end{array}$ \\
\hline & " tariff levels & & & & & & & $\begin{array}{l}0.005 * * \\
(0.002)\end{array}$ & $\begin{array}{r}-0.003 \\
(0.006)\end{array}$ & $\begin{array}{l}0.005 * \\
(0.002)\end{array}$ & $\begin{array}{c}-0.003 \\
(0.006)\end{array}$ & $\begin{array}{r}0.001 \\
(0.002)\end{array}$ & $\begin{array}{r}0.000 \\
(0.009)\end{array}$ & $\begin{array}{r}0.002 \\
(0.003)\end{array}$ & $\begin{array}{c}0.013 * \\
(0.008)\end{array}$ & $\begin{array}{r}0.002 \\
(0.003)\end{array}$ & $\begin{array}{r}0.004 \\
(0.008)\end{array}$ \\
\hline & " log distance & & & & & & & $\begin{array}{r}-0.001 \\
(0.001)\end{array}$ & $\begin{array}{l}0.005 * * \\
(0.003)\end{array}$ & $\begin{array}{r}-0.001 \\
(0.001)\end{array}$ & $\begin{array}{r}0.008 \\
(0.005)\end{array}$ & $\begin{array}{r}-0.002 \\
(0.002)\end{array}$ & $\begin{array}{r}0.013 \\
(0.010)\end{array}$ & $\begin{array}{r}-0.003 \\
(0.002)\end{array}$ & $\begin{array}{r}-0.012 \\
(0.008)\end{array}$ & $\begin{array}{l}-0.004 * * \\
(0.002)\end{array}$ & $\begin{array}{l}-0.003 \\
(0.007)\end{array}$ \\
\hline & " $\log [$ RGDP(importer)/RGDP(exporter)] & & & & & & & & & $\begin{array}{r}0.001 \\
(0.003)\end{array}$ & $\begin{array}{r}0.007 \\
(0.009)\end{array}$ & $\begin{array}{r}0.002 \\
(0.003)\end{array}$ & $\begin{array}{r}0.020 \\
(0.018)\end{array}$ & $\begin{array}{r}0.001 \\
(0.003)\end{array}$ & $\begin{array}{r}-0.017 \\
(0.012)\end{array}$ & $\begin{array}{r}-0.002 \\
(0.002)\end{array}$ & $\begin{array}{r}0.003 \\
(0.012)\end{array}$ \\
\hline & $* \log$ real wage $(\$)$ & & & & & & & & & & & $\begin{array}{l}-0.017 * * * \\
(0.003)\end{array}$ & $\begin{array}{r}-0.031 \\
(0.027)\end{array}$ & $\begin{array}{l}-0.014 * * * \\
(0.004)\end{array}$ & $\begin{array}{r}-0.020 \\
(0.020)\end{array}$ & $\begin{array}{l}-0.013 * * * \\
(0.003)\end{array}$ & $\begin{array}{l}-0.030 * \\
(0.018)\end{array}$ \\
\hline & * long term inflation & & & & & & & & & & & & & $\begin{array}{l}0.135 * \\
(0.078)\end{array}$ & $\begin{array}{l}-0.052 \\
(0.080)\end{array}$ & $\begin{array}{l}0.137 * \\
(0.083)\end{array}$ & $\begin{array}{r}-0.062 \\
(0.085)\end{array}$ \\
\hline & " long term exchange rate variability & & & & & & & & & & & & & $\begin{array}{r}-0.016 \\
(0.015)\end{array}$ & $\begin{array}{l}-0.230 * * \\
(0.102)\end{array}$ & $\begin{array}{r}-0.005 \\
(0.014)\end{array}$ & $\begin{array}{l}-0.257 * * * \\
(0.073)\end{array}$ \\
\hline Ecm & "stage of business cycle & & & & & & & & & & & & & & & $\begin{array}{r}0.039 \\
(0.027)\end{array}$ & $\begin{array}{r}0.023 \\
(0.049)\end{array}$ \\
\hline & $\begin{array}{l}\text { \# of Observations } \\
\text { Ad usted R-squared }\end{array}$ & $\begin{array}{c}732 \\
0.771\end{array}$ & & $\begin{array}{c}516 \\
0.814\end{array}$ & & $\begin{array}{r}516 \\
0.814\end{array}$ & & $\begin{array}{r}425 \\
0.816\end{array}$ & & $\begin{array}{c}425 \\
0.816\end{array}$ & & $\begin{array}{c}299 \\
0.879\end{array}$ & & $\begin{array}{r}297 \\
0.925\end{array}$ & & $\begin{array}{r}295 \\
0.939\end{array}$ & \\
\hline
\end{tabular}


Appendix Table 16. Determination of Passthrough to Unit Values: Developing countries, Relative to Rich countries

\begin{tabular}{|c|c|c|c|c|c|c|c|c|c|c|c|c|c|c|c|c|c|}
\hline \multirow[b]{3}{*}{ change in } & \multirow[b]{3}{*}{ exchange rate } & \multicolumn{2}{|c|}{ Equation 1} & \multirow{2}{*}{\multicolumn{2}{|c|}{$\begin{array}{c}\text { Equation2 } \\
\text { D Dev }\end{array}$}} & \multirow{2}{*}{\multicolumn{2}{|c|}{\begin{tabular}{l}
\multicolumn{2}{c}{ Equation 3} \\
Rich D Dev.
\end{tabular}}} & \multirow{2}{*}{\multicolumn{2}{|c|}{\begin{tabular}{c}
\multicolumn{2}{c}{ Equation 4} \\
Rich $\quad$ D Dev.
\end{tabular}}} & \multirow{2}{*}{\multicolumn{2}{|c|}{$\begin{array}{l}\text { Equation } 5 \\
\text { Rich } \quad \text { D Dev. }\end{array}$}} & \multicolumn{2}{|c|}{ Equation 6} & \multicolumn{2}{|c|}{ Equation 7} & \multicolumn{2}{|c|}{$\begin{array}{l}\text { Equation } 8 \\
\end{array}$} \\
\hline & & Rich & D Dev. & & & & & & & & & Rich & D Dev. & Rich & D Dev. & Rich & D Dev. \\
\hline & & $\begin{array}{l}0.320{ }^{*} \\
(0.195)\end{array}$ & $\begin{array}{l}-0.708 * \\
(0.376)\end{array}$ & $\begin{array}{r}0.208 \\
(0.305)\end{array}$ & $\begin{array}{l}-1.100{ }^{* *} \\
(0.495)\end{array}$ & $\begin{array}{l}0.130 * * * * \\
(0.004)\end{array}$ & $\begin{array}{l}-1.006 * * \\
(0.500)\end{array}$ & $\begin{array}{l}1.376 \\
(1.191)\end{array}$ & $\begin{array}{r}0.802 \\
(1.545)\end{array}$ & $\begin{array}{l}1.300 \\
(1.153)\end{array}$ & $\begin{array}{r}0.896 \\
(1.516)\end{array}$ & $\begin{array}{r}1.123 \\
(1.200)\end{array}$ & $\begin{array}{r}-0.963 \\
(1.642)\end{array}$ & $\begin{array}{r}0.562 \\
(1.646)\end{array}$ & $\begin{array}{r}0.519 \\
(2.024)\end{array}$ & $\begin{array}{r}1.351 \\
(1.545)\end{array}$ & $\begin{array}{r}-0.688 \\
(1.957)\end{array}$ \\
\hline & depreciations & $\begin{array}{l}0.703 * * \\
(0.288)\end{array}$ & $\begin{array}{r}0.621 \\
(0.517)\end{array}$ & $\begin{array}{l}0.782 * * * \\
(0.287)\end{array}$ & $\begin{array}{r}0.642 \\
(0.515)\end{array}$ & $\begin{array}{l}0.861 * * * \\
(0.004)\end{array}$ & $\begin{array}{r}0.599 \\
(0.520)\end{array}$ & $\begin{array}{l}0.6511^{* *} \\
(0.312)\end{array}$ & $\begin{array}{r}0.869 \\
(0.570)\end{array}$ & $\begin{array}{r}0.584 \\
(0.362)\end{array}$ & $\begin{array}{r}0.785 \\
(0.595)\end{array}$ & $\begin{array}{r}0.448 \\
(0.379)\end{array}$ & $\begin{array}{r}0.652 \\
(0.687)\end{array}$ & $\begin{array}{r}0.757 \\
(0.498)\end{array}$ & $\begin{array}{r}0.232 \\
(0.752)\end{array}$ & $\begin{array}{r}0.771 \\
(0.503)\end{array}$ & $\begin{array}{r}0.452 \\
(0.765)\end{array}$ \\
\hline & depreciations above $25 \%$ & $\begin{array}{l}-0.548 * * \\
(0.237)\end{array}$ & $\begin{array}{r}0.469 \\
(0.310)\end{array}$ & $\begin{array}{l}-0.664 * * * \\
(0.248)\end{array}$ & $\begin{array}{l}0.619 * \\
(0.318)\end{array}$ & $\begin{array}{l}-0.652 * * * \\
(0.252)\end{array}$ & $\begin{array}{c}0.621{ }^{*} \\
(0.321)\end{array}$ & $\begin{array}{l}-0.732 * * * \\
(0.276)\end{array}$ & $\begin{array}{r}0.568 \\
(0.352)\end{array}$ & $\begin{array}{l}-0.689 * * \\
(0.293)\end{array}$ & $\begin{array}{r}0.555 \\
(0.365)\end{array}$ & $\begin{array}{l}-0.702 * * \\
(0.305)\end{array}$ & $\begin{array}{r}0.448 \\
(0.399)\end{array}$ & $\begin{array}{l}-0.787 * * \\
(0.329)\end{array}$ & $\begin{array}{r}0.651 \\
(0.429)\end{array}$ & $\begin{array}{l}-0.616 * \\
(0.347)\end{array}$ & $\begin{array}{r}0.368 \\
(0.452)\end{array}$ \\
\hline change in & exporter's priee & $\begin{array}{l}0.234 * * * \\
(0.070)\end{array}$ & $\begin{array}{l}-0.276 * * \\
(0.117)\end{array}$ & $\begin{array}{l}0.340 * * * \\
(0.078)\end{array}$ & $\begin{array}{l}-0.384 * * * \\
(0.123)\end{array}$ & $\begin{array}{l}0.339 * * * \\
(0.078)\end{array}$ & $\begin{array}{l}-0.382 * * * \\
(0.123)\end{array}$ & $\begin{array}{l}0.370 * * * \\
(0.088)\end{array}$ & $\begin{array}{l}-0.450 * * * \\
(0.137)\end{array}$ & $\begin{array}{l}0.367 * * * \\
(0.090)\end{array}$ & $\begin{array}{l}-0.442 * * * \\
(0.138)\end{array}$ & $\begin{array}{l}0.350 * * * \\
(0.092)\end{array}$ & $\begin{array}{r}-0.183 \\
(0.161)\end{array}$ & $\begin{array}{l}0.349 * * * \\
(0.090)\end{array}$ & $\begin{array}{r}-0.188 \\
(0.167)\end{array}$ & $\begin{array}{l}0.348 * * * \\
(0.090)\end{array}$ & $\begin{array}{r}-0.199 \\
(0.165)\end{array}$ \\
\hline$\left(\right.$ delta s) ${ }^{*}$ & trend & & & $\begin{array}{r}0.018 \\
(0.027)\end{array}$ & $\begin{array}{r}0.038 \\
(0.035)\end{array}$ & $\begin{array}{r}0.021 \\
(0.028)\end{array}$ & $\begin{array}{r}0.038 \\
(0.035)\end{array}$ & $\begin{array}{r}0.020 \\
(0.030)\end{array}$ & $\begin{array}{r}0.050 \\
(0.038)\end{array}$ & $\begin{array}{r}0.023 \\
(0.030)\end{array}$ & $\begin{array}{r}0.049 \\
(0.038)\end{array}$ & $\begin{array}{r}0.033 \\
(0.029)\end{array}$ & $\begin{array}{r}0.038 \\
(0.045)\end{array}$ & $\begin{array}{r}-0.003 \\
(0.051)\end{array}$ & $\begin{array}{r}0.046 \\
(0.064)\end{array}$ & $\begin{array}{r}-0.025 \\
(0.055)\end{array}$ & $\begin{array}{r}0.065 \\
(0.068)\end{array}$ \\
\hline$\left(\right.$ delta s) ${ }^{*}$ & $\begin{array}{l}\text { log[per capitaRGDP(importer) / } \\
\text { per capitaRGDP(exporter)] }\end{array}$ & & & & & $\begin{array}{l}0.039 * \\
(0.020)\end{array}$ & $\begin{array}{r}0.000 \\
(0.027)\end{array}$ & $\begin{array}{r}0.015 \\
(0.021)\end{array}$ & $\begin{array}{l}0.049 * \\
(0.028)\end{array}$ & $\begin{array}{r}0.054 \\
(0.060)\end{array}$ & $\begin{array}{r}0.029 \\
(0.077)\end{array}$ & $\begin{array}{r}0.061 \\
(0.066)\end{array}$ & $\begin{array}{r}0.072 \\
(0.104)\end{array}$ & $\begin{array}{l}0.185 * * * \\
(0.062)\end{array}$ & $\begin{array}{r}-0.053 \\
(0.123)\end{array}$ & $\begin{array}{l}0.214 * * * \\
(0.067)\end{array}$ & $\begin{array}{r}-0.095 \\
(0.128)\end{array}$ \\
\hline$\left(\right.$ delta s) ${ }^{*}$ & tariff levels & & & & & & & $\begin{array}{r}0.040 \\
(0.218)\end{array}$ & $\begin{array}{r}0.053 \\
(0.256)\end{array}$ & $\begin{array}{r}0.058 \\
(0.207)\end{array}$ & $\begin{array}{r}0.045 \\
(0.256)\end{array}$ & $\begin{array}{r}0.072 \\
(0.219)\end{array}$ & $\begin{array}{r}-0.045 \\
(0.284)\end{array}$ & $\begin{array}{r}0.193 \\
(0.269)\end{array}$ & $\begin{array}{l}-0.197 \\
(0.330)\end{array}$ & $\begin{array}{r}0.152 \\
(0.258)\end{array}$ & $\begin{array}{r}-0.093 \\
(0.325)\end{array}$ \\
\hline$\left(\right.$ delta s) ${ }^{*}$ & $\log$ distance & & & & & & & $\begin{array}{r}-0.126 \\
(0.128)\end{array}$ & $\begin{array}{r}-0.239 \\
(0.162)\end{array}$ & $\begin{array}{r}-0.130 \\
(0.128)\end{array}$ & $\begin{array}{r}-0.231 \\
(0.163)\end{array}$ & $\begin{array}{r}-0.111 \\
(0.130)\end{array}$ & $\begin{array}{r}0.009 \\
(0.174)\end{array}$ & $\begin{array}{r}-0.110 \\
(0.177)\end{array}$ & $\begin{array}{r}-0.052 \\
(0.212)\end{array}$ & $\begin{array}{r}-0.194 \\
(0.171)\end{array}$ & $\begin{array}{r}0.066 \\
(0.207)\end{array}$ \\
\hline$\left(\right.$ delta s) ${ }^{*}$ & $\log [\operatorname{RGDP}($ importer)/RGDP(exporter)] & & & & & & & & & $\begin{array}{r}-0.042 \\
(0.061)\end{array}$ & $\begin{array}{r}0.022 \\
(0.078)\end{array}$ & $\begin{array}{r}-0.047 \\
(0.070)\end{array}$ & $\begin{array}{r}-0.095 \\
(0.101)\end{array}$ & $\begin{array}{l}-0.159 * * * \\
(0.060)\end{array}$ & $\begin{array}{r}0.004 \\
(0.112)\end{array}$ & $\begin{array}{l}-0.189 * * * \\
(0.068)\end{array}$ & $\begin{array}{r}0.047 \\
(0.119)\end{array}$ \\
\hline$\left(\right.$ delta s) ${ }^{*}$ & log real wage $(\$)$ & & & & & & & & & & & $\begin{array}{r}-0.007 \\
(0.024)\end{array}$ & $\begin{array}{r}0.009 \\
(0.031)\end{array}$ & $\begin{array}{r}-0.060 \\
(0.059)\end{array}$ & $\begin{array}{r}0.066 \\
(0.064)\end{array}$ & $\begin{array}{r}-0.057 \\
(0.058)\end{array}$ & $\begin{array}{r}0.064 \\
(0.063)\end{array}$ \\
\hline$\left(\right.$ delta s) ${ }^{*}$ & long term inflation & & & & & & & & & & & & & $\begin{array}{r}-10.441 \\
(10.097)\end{array}$ & $\begin{array}{r}9.643 \\
(10.099)\end{array}$ & $\begin{array}{r}-12.491 \\
(10.685)\end{array}$ & $\begin{array}{r}11.597 \\
(10.687)\end{array}$ \\
\hline$\left(\right.$ delta s) ${ }^{*}$ & long term exchange rate variability & & & & & & & & & & & & & $\begin{array}{l}18.566 * \\
(10.348)\end{array}$ & $\begin{array}{r}-16.418 \\
(10.515)\end{array}$ & $\begin{array}{l}24.965 * * \\
(11.832)\end{array}$ & $\begin{array}{l}-24.108 * * \\
(11.969)\end{array}$ \\
\hline$\left(\right.$ delta s) ${ }^{*}$ & US Importer dummy & & & & & & & & & & & & & & & $\begin{array}{r}0.023 \\
(0.275)\end{array}$ & \\
\hline$\left(\right.$ delta s) ${ }^{*}$ & stage of business cycle & & & & & & & & & & & & & & & $\begin{array}{r}4.641 \\
(2.936)\end{array}$ & $\begin{array}{l}-8.095 * * \\
(3.392)\end{array}$ \\
\hline & Error correction term (Ecm) & $\begin{array}{r}-0.216 \\
(0.036)\end{array}$ & $\begin{array}{r}-0.008 \\
(0.017)\end{array}$ & $\begin{array}{l}-0.148 * * * \\
(0.035)\end{array}$ & $\begin{array}{l}-0.085 * * \\
(0.035)\end{array}$ & $\begin{array}{l}-0.150 * * * \\
(0.035)\end{array}$ & $\begin{array}{l}-0.096 * * \\
(0.038)\end{array}$ & $\begin{array}{r}0.185 \\
(0.144)\end{array}$ & $\begin{array}{l}-0.382 * \\
(0.223)\end{array}$ & $\begin{array}{r}0.173 \\
(0.145)\end{array}$ & $\begin{array}{l}-0.418 * \\
(0.224)\end{array}$ & $\begin{array}{l}0.239^{*} \\
(0.131)\end{array}$ & $\begin{array}{r}-0.181 \\
(0.194)\end{array}$ & $\begin{array}{l}0.181 * \\
(0.102)\end{array}$ & $\begin{array}{r}-0.074 \\
(0.182)\end{array}$ & $\begin{array}{r}0.151 \\
(0.103)\end{array}$ & $\begin{array}{r}-0.052 \\
(0.186)\end{array}$ \\
\hline & * trend & & & $\begin{array}{l}-0.011 * * * \\
(0.003)\end{array}$ & $\begin{array}{l}0.012 * * * \\
(0.004)\end{array}$ & $\begin{array}{l}-0.011 * * * \\
(0.003)\end{array}$ & $\begin{array}{l}0.012 * * * \\
(0.004)\end{array}$ & $\begin{array}{l}-0.010 * * * \\
(0.003)\end{array}$ & $\begin{array}{l}0.009 * \\
(0.005)\end{array}$ & $\begin{array}{l}-0.009 * * \\
(0.004)\end{array}$ & $\begin{array}{r}0.009 \\
(0.005)\end{array}$ & $\begin{array}{l}-0.010 * * \\
(0.004)\end{array}$ & $\begin{array}{l}0.011{ }^{*} \\
(0.006)\end{array}$ & $\begin{array}{l}-0.006 * \\
(0.004)\end{array}$ & $\begin{array}{r}0.007 \\
(0.006)\end{array}$ & $\begin{array}{r}-0.006 \\
(0.004)\end{array}$ & $\begin{array}{r}0.006 \\
(0.006)\end{array}$ \\
\hline & $\begin{array}{l}* \log [\text { per capitaRGDP(importer) } / \\
\text { per capitaRGDP(exporter)] }\end{array}$ & & & & & $\begin{array}{r}-0.003 \\
(0.003)\end{array}$ & $\begin{array}{r}-0.001 \\
(0.004)\end{array}$ & $\begin{array}{r}-0.001 \\
(0.005)\end{array}$ & $\begin{array}{r}-0.001 \\
(0.006)\end{array}$ & $\begin{array}{l}-0.011 * \\
(0.006)\end{array}$ & $\begin{array}{l}0.020 * * \\
(0.010)\end{array}$ & $\begin{array}{r}-0.006 \\
(0.009)\end{array}$ & $\begin{array}{r}0.011 \\
(0.017)\end{array}$ & $\begin{array}{r}-0.006 \\
(0.008)\end{array}$ & $\begin{array}{r}0.008 \\
(0.018)\end{array}$ & $\begin{array}{r}-0.008 \\
(0.008)\end{array}$ & $\begin{array}{r}0.012 \\
(0.018)\end{array}$ \\
\hline & * tariff levels & & & & & & & $\begin{array}{l}0.052 * * * \\
(0.020)\end{array}$ & $\begin{array}{r}-0.038 \\
(0.025)\end{array}$ & $\begin{array}{l}0.052 * * * \\
(0.020)\end{array}$ & $\begin{array}{r}-0.033 \\
(0.025)\end{array}$ & $\begin{array}{l}0.042 * * \\
(0.019)\end{array}$ & $\begin{array}{r}-0.013 \\
(0.027)\end{array}$ & $\begin{array}{r}0.035 \\
(0.022)\end{array}$ & $\begin{array}{r}-0.007 \\
(0.030)\end{array}$ & $\begin{array}{c}0.037 * \\
(0.022)\end{array}$ & $\begin{array}{r}-0.007 \\
(0.030)\end{array}$ \\
\hline & * log distance & & & & & & & $\begin{array}{l}-0.054 * * * \\
(0.016)\end{array}$ & $\begin{array}{l}0.046 * \\
(0.023)\end{array}$ & $\begin{array}{l}-0.050 * * * \\
(0.018)\end{array}$ & $\begin{array}{c}0.043 * \\
(0.025)\end{array}$ & $\begin{array}{l}-0.056 * * \\
(0.022)\end{array}$ & $\begin{array}{r}0.029 \\
(0.026)\end{array}$ & $\begin{array}{l}-0.051 * * * \\
(0.018)\end{array}$ & $\begin{array}{r}0.020 \\
(0.025)\end{array}$ & $\begin{array}{l}-0.048 * * * \\
(0.018)\end{array}$ & $\begin{array}{r}0.017 \\
(0.024)\end{array}$ \\
\hline & * $\log [$ RGDP(importer)/RGDP(exporter)] & & & & & & & & & $\begin{array}{r}0.011 \\
(0.009)\end{array}$ & $\begin{array}{l}-0.023 * \\
(0.012)\end{array}$ & $\begin{array}{r}0.008 \\
(0.008)\end{array}$ & $\begin{array}{r}-0.007 \\
(0.016)\end{array}$ & $\begin{array}{r}0.009 \\
(0.008)\end{array}$ & $\begin{array}{r}-0.005 \\
(0.016)\end{array}$ & $\begin{array}{r}0.011 \\
(0.007)\end{array}$ & $\begin{array}{r}-0.009 \\
(0.016)\end{array}$ \\
\hline & $*$ log real wage $(\$)$ & & & & & & & & & & & $\begin{array}{r}-0.022 \\
(0.030)\end{array}$ & $\begin{array}{r}0.040 \\
(0.034)\end{array}$ & $\begin{array}{r}-0.019 \\
(0.026)\end{array}$ & $\begin{array}{r}0.038 \\
(0.032)\end{array}$ & $\begin{array}{r}-0.022 \\
(0.026)\end{array}$ & $\begin{array}{r}0.039 \\
(0.032)\end{array}$ \\
\hline & * long term inflation & & & & & & & & & & & & & $\begin{array}{r}0.558 \\
(0.605)\end{array}$ & $\begin{array}{r}-0.573 \\
(0.608)\end{array}$ & $\begin{array}{r}0.567 \\
(0.610)\end{array}$ & $\begin{array}{r}-0.574 \\
(0.613)\end{array}$ \\
\hline & * long term exchange rate variability & & & & & & & & & & & & & $\begin{array}{r}-0.375 \\
(0.314)\end{array}$ & $\begin{array}{r}0.443 \\
(0.333)\end{array}$ & $\begin{array}{r}-0.380 \\
(0.316)\end{array}$ & $\begin{array}{r}0.444 \\
(0.335)\end{array}$ \\
\hline & * US Importer dummy & & & & & & & & & & & & & & & $\begin{array}{r}0.068 \\
(0.087)\end{array}$ & \\
\hline Ecm & * stage of business cycle & & & & & & & & & & & & & & & $\begin{array}{r}-0.212 \\
(0.248)\end{array}$ & $\begin{array}{r}0.240 \\
(0.330)\end{array}$ \\
\hline & $\begin{array}{l}\text { \# of Observations } \\
\text { Adjusted R-squared }\end{array}$ & $\begin{array}{l}3774 \\
0.156\end{array}$ & & $\begin{array}{l}3774 \\
0.161\end{array}$ & & $\begin{array}{l}3774 \\
0.162\end{array}$ & & 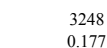 & & $\begin{array}{l}3248 \\
0.178\end{array}$ & & $\begin{array}{l}2423 \\
0.166\end{array}$ & & $\begin{array}{l}2389 \\
0.173\end{array}$ & & $\begin{array}{l}2381 \\
0.175\end{array}$ & \\
\hline
\end{tabular}


Table a1: Pass-through to Import price of Marlboro

\begin{tabular}{|c|c|c|c|c|c|c|c|c|c|c|}
\hline change in & exchange rate & $\begin{array}{c}\text { Eqn 1 } \\
0.981 * * * \\
(0.008)\end{array}$ & $\begin{array}{c}\text { Eqn2 } \\
0.990 \\
(0.018)\end{array}$ & $\begin{array}{c}\text { Eqn3 } \\
0.946^{* * *} \\
(0.038)\end{array}$ & $\begin{array}{c}\text { Eqn } 4 \\
1.040^{* * *} \\
(0.237)\end{array}$ & $\begin{array}{c}\text { Eqn 5 } \\
1.050 * * * \\
(0.245)\end{array}$ & $\begin{array}{r}\text { Eqn 6 } \\
-1.090 \\
(1.347)\end{array}$ & $\begin{array}{r}\text { Eqn } 7 \\
0.936 \\
(1.455)\end{array}$ & $\begin{array}{r}\text { Eqn 8 } \\
0.936 \\
(1.455)\end{array}$ & $\begin{array}{c}\text { Eqn } 9 \\
-1.009 \\
(1.305)\end{array}$ \\
\hline change in & exporter's price & $\begin{array}{r}-0.044 \\
(0.046)\end{array}$ & $\begin{array}{r}-0.016 \\
(0.046)\end{array}$ & $\begin{array}{r}-0.016 \\
(0.045)\end{array}$ & $\begin{array}{r}-0.023 \\
(0.051)\end{array}$ & $\begin{array}{r}-0.019 \\
(0.052)\end{array}$ & $\begin{array}{l}-0.009 \\
(0.059)\end{array}$ & $\begin{array}{r}-0.044 \\
(0.050)\end{array}$ & $\begin{array}{l}-0.044 \\
(0.050)\end{array}$ & $\begin{array}{r}0.007 \\
(0.059)\end{array}$ \\
\hline$($ delta s)* & trend & & $\begin{array}{l}-0.002 \\
(0.003)\end{array}$ & $\begin{array}{r}-0.007 \\
(0.006)\end{array}$ & $\begin{array}{l}-0.013 \\
(0.009)\end{array}$ & $\begin{array}{r}-0.011 \\
(0.009)\end{array}$ & $\begin{array}{l}-0.060 * * * \\
(0.014)\end{array}$ & $\begin{array}{l}-0.062^{* * *} \\
(0.018)\end{array}$ & $\begin{array}{l}-0.062 * * * \\
(0.018)\end{array}$ & $\begin{array}{l}-0.065^{* * *} \\
(0.013)\end{array}$ \\
\hline$($ delta s)* & $\begin{array}{l}\log [\text { per capitaRGDP(importer) / } \\
\text { per capitaRGDP(exporter)] }\end{array}$ & & & $\begin{array}{l}-0.053 \\
(0.045)\end{array}$ & $\begin{array}{r}-0.080 \\
(0.075)\end{array}$ & $\begin{array}{l}-0.075 \\
(0.073)\end{array}$ & $\begin{array}{l}-0.317^{* * *} \\
(0.086)\end{array}$ & $\begin{array}{l}-0.432 * * * \\
(0.064)\end{array}$ & $\begin{array}{l}-0.432 * * * \\
(0.064)\end{array}$ & $\begin{array}{l}-0.337^{* * *} \\
(0.090)\end{array}$ \\
\hline$($ delta s)* & tariff levels & & & & $\begin{array}{r}-0.032 \\
(0.023)\end{array}$ & $\begin{array}{r}-0.034 \\
(0.024)\end{array}$ & $\begin{array}{l}-0.139 * * * \\
(0.041)\end{array}$ & $\begin{array}{l}-0.218 * * * \\
(0.073)\end{array}$ & $\begin{array}{l}-0.218 * * * \\
(0.073)\end{array}$ & $\begin{array}{l}-0.109 * \\
(0.066)\end{array}$ \\
\hline$($ delta s)* & $\log$ distance & & & & $\begin{array}{r}-0.004 \\
(0.024)\end{array}$ & $\begin{array}{r}-0.002 \\
(0.025)\end{array}$ & $\begin{array}{l}0.287 * \\
(0.155)\end{array}$ & $\begin{array}{r}0.020 \\
(0.170)\end{array}$ & $\begin{array}{r}0.020 \\
(0.170)\end{array}$ & $\begin{array}{c}0.280 * \\
(0.155)\end{array}$ \\
\hline$($ delta s)* & $\log [$ RGDP(importer)/RGDP(exporter)] & & & & & $\begin{array}{r}0.008 \\
(0.009)\end{array}$ & $\begin{array}{l}0.106 \text { *** } \\
(0.040)\end{array}$ & $\begin{array}{r}0.035 \\
(0.059)\end{array}$ & $\begin{array}{r}0.035 \\
(0.059)\end{array}$ & $\begin{array}{l}0.117^{* * *} \\
(0.035)\end{array}$ \\
\hline$($ delta s)* & log real wage $(\$)$ & & & & & & $\begin{array}{l}-0.028 \text { *** } \\
(0.009)\end{array}$ & $\begin{array}{l}-0.036^{* * *} \\
(0.006)\end{array}$ & $\begin{array}{l}-0.036 \text { *** } \\
(0.006)\end{array}$ & $\begin{array}{l}-0.029 * * * \\
(0.008)\end{array}$ \\
\hline$($ delta s)* & long term inflation & & & & & & & $\begin{array}{r}0.046 \\
(0.043)\end{array}$ & $\begin{array}{r}0.046 \\
(0.043)\end{array}$ & $\begin{array}{r}-0.028 \\
(0.039)\end{array}$ \\
\hline$($ delta s)* & long term exchange rate variability & & & & & & & $\begin{array}{l}-1.327 * * \\
(0.631)\end{array}$ & $\begin{array}{l}-1.327 * * \\
(0.631)\end{array}$ & \\
\hline$($ delta s)* & US Importer dummy & & & & & & & & & \\
\hline & Error Correction term (ECM) & $\begin{array}{l}-0.180 \text { *** } \\
(0.025)\end{array}$ & $\begin{array}{l}-0.315 * * * \\
(0.050)\end{array}$ & $\begin{array}{l}-0.249 \text { *** } \\
(0.051)\end{array}$ & $\begin{array}{r}-0.771 \\
(0.527)\end{array}$ & $\begin{array}{r}-0.813 \\
(0.533)\end{array}$ & $\begin{array}{r}0.134 \\
(0.610)\end{array}$ & $\begin{array}{r}0.054 \\
(0.647)\end{array}$ & $\begin{array}{r}0.054 \\
(0.647)\end{array}$ & $\begin{array}{r}0.109 \\
(0.603)\end{array}$ \\
\hline $\mathrm{ECM}$ & trend & & $\begin{array}{l}0.013 \text { *** } \\
(0.004)\end{array}$ & $\begin{array}{l}0.015^{* * *} \\
(0.004)\end{array}$ & $\begin{array}{l}0.0144^{* * *} \\
(0.004)\end{array}$ & $\begin{array}{l}0.015 \text { *** } \\
(0.004)\end{array}$ & $\begin{array}{l}0.020^{* * *} \\
(0.005)\end{array}$ & $\begin{array}{l}0.009 \text { ** } \\
(0.004)\end{array}$ & $\begin{array}{l}0.009 \text { ** } \\
(0.004)\end{array}$ & $\begin{array}{l}0.017^{* * *} \\
(0.005)\end{array}$ \\
\hline ECM & $\begin{array}{l}\log [\text { per capitaRGDP(importer) / } \\
\text { per capitaRGDP(exporter)] }\end{array}$ & & & $\begin{array}{l}0.065 * * * \\
(0.023)\end{array}$ & $\begin{array}{l}0.085^{* *} \\
(0.034)\end{array}$ & $\begin{array}{l}0.098 * * * \\
(0.034)\end{array}$ & $\begin{array}{l}0.085 * \\
(0.045)\end{array}$ & $\begin{array}{r}0.059 \\
(0.043)\end{array}$ & $\begin{array}{r}0.059 \\
(0.043)\end{array}$ & $\begin{array}{r}0.068 \\
(0.044)\end{array}$ \\
\hline $\mathrm{ECM}$ & tariff levels & & & & $\begin{array}{r}-0.004 \\
(0.032)\end{array}$ & $\begin{array}{r}0.006 \\
(0.032)\end{array}$ & $\begin{array}{r}0.001 \\
(0.039)\end{array}$ & $\begin{array}{r}-0.021 \\
(0.038)\end{array}$ & $\begin{array}{l}-0.021 \\
(0.038)\end{array}$ & $\begin{array}{l}-0.011 \\
(0.038)\end{array}$ \\
\hline $\mathrm{ECM}$ & $\log$ distance & & & & $\begin{array}{r}0.065 \\
(0.060)\end{array}$ & $\begin{array}{r}0.055 \\
(0.062)\end{array}$ & $\begin{array}{r}-0.051 \\
(0.072)\end{array}$ & $\begin{array}{r}-0.010 \\
(0.073)\end{array}$ & $\begin{array}{r}-0.010 \\
(0.073)\end{array}$ & $\begin{array}{r}-0.048 \\
(0.071)\end{array}$ \\
\hline $\mathrm{ECM}$ & $\log [$ RGDP(importer)/RGDP(exporter)] & & & & & $\begin{array}{l}-0.029 \\
(0.020)\end{array}$ & $\begin{array}{r}-0.032 \\
(0.028)\end{array}$ & $\begin{array}{r}0.007 \\
(0.025)\end{array}$ & $\begin{array}{r}0.007 \\
(0.025)\end{array}$ & $\begin{array}{r}-0.031 \\
(0.028)\end{array}$ \\
\hline $\mathrm{ECM}$ & log real wage $(\$)$ & & & & & & $\begin{array}{r}-0.013 \\
(0.010)\end{array}$ & $\begin{array}{r}-0.009 \\
(0.008)\end{array}$ & $\begin{array}{l}-0.009 \\
(0.008)\end{array}$ & $\begin{array}{r}-0.003 \\
(0.011)\end{array}$ \\
\hline $\mathrm{ECM}$ & long term inflation & & & & & & & $\begin{array}{l}0.175^{* *} \\
(0.073)\end{array}$ & $\begin{array}{l}0.175 \text { ** } \\
(0.073)\end{array}$ & $\begin{array}{l}-0.110 * \\
(0.065)\end{array}$ \\
\hline $\mathrm{ECM}$ & long term exchange rate variability & & & & & & & $\begin{array}{l}-4.717^{* * *} \\
(0.923)\end{array}$ & $\begin{array}{l}-4.717^{* * *} \\
(0.923)\end{array}$ & \\
\hline
\end{tabular}

ECM* US Importer dummy

\begin{tabular}{|c|c|c|c|c|c|c|c|c|c|}
\hline \# of Observations & 782 & 782 & 782 & 636 & 636 & 383 & 374 & 374 & 383 \\
\hline Adjusted R-squared & 0.958 & 0.959 & 0.960 & 0.961 & 0.961 & 0.972 & 0.974 & 0.974 & 0.972 \\
\hline Country dummies & yes & yes & yes & yes & yes & yes & yes & yes & yes \\
\hline Product dummies & yes & yes & yes & yes & yes & yes & yes & yes & yes \\
\hline
\end{tabular}


Table a2: Pass-through to Import price of Coca-Cola

\begin{tabular}{|c|c|c|c|c|c|c|c|c|c|c|}
\hline change in & exchange rate & $\begin{array}{c}\text { Eqn 1 } \\
0.999 * * * \\
(0.009)\end{array}$ & $\begin{array}{c}\text { Eqn2 } \\
0.998 \\
(0.019)\end{array}$ & $\begin{array}{c}\text { Eqn3 } \\
0.995 \text { *** } \\
(0.043)\end{array}$ & $\begin{array}{c}\text { Eqn } 4 \\
1.790 \text { *** } \\
(0.294)\end{array}$ & $\begin{array}{c}\text { Eqn 5 } \\
1.805 \text { *** } \\
(0.301)\end{array}$ & $\begin{array}{c}\text { Eqn 6 } \\
2.548 * \\
(1.533)\end{array}$ & $\begin{array}{r}\text { Eqn } 7 \\
0.267 \\
(1.312)\end{array}$ & $\begin{array}{r}\text { Eqn 8 } \\
0.267 \\
(1.312)\end{array}$ & $\begin{array}{c}\text { Eqn 9 } \\
2.813 * \\
(1.454)\end{array}$ \\
\hline change in & exporter's price & $\begin{array}{l}0.237 * * * \\
(0.048)\end{array}$ & $\begin{array}{l}0.237^{* * *} \\
(0.048)\end{array}$ & $\begin{array}{l}0.234^{* * *} \\
(0.049)\end{array}$ & $\begin{array}{l}0.215^{* * *} \\
(0.054)\end{array}$ & $\begin{array}{l}0.215^{* * *} \\
(0.054)\end{array}$ & $\begin{array}{r}0.087 \\
(0.055)\end{array}$ & $\begin{array}{r}0.043 \\
(0.050)\end{array}$ & $\begin{array}{r}0.043 \\
(0.050)\end{array}$ & $\begin{array}{r}0.080 \\
(0.053)\end{array}$ \\
\hline$($ delta s)* & trend & & $\begin{array}{r}0.000 \\
(0.003)\end{array}$ & $\begin{array}{r}0.000 \\
(0.006)\end{array}$ & $\begin{array}{r}-0.009 \\
(0.008)\end{array}$ & $\begin{array}{r}-0.008 \\
(0.009)\end{array}$ & $\begin{array}{l}-0.047^{* * *} \\
(0.013)\end{array}$ & $\begin{array}{l}-0.044^{* * *} \\
(0.017)\end{array}$ & $\begin{array}{l}-0.044 * * * \\
(0.017)\end{array}$ & $\begin{array}{l}-0.047 \text { ** } \\
(0.013)\end{array}$ \\
\hline$($ delta s)* & $\begin{array}{l}\log [\text { per capitaRGDP(importer) / } \\
\text { per capitaRGDP(exporter)] }\end{array}$ & & & $\begin{array}{r}-0.003 \\
(0.046)\end{array}$ & $\begin{array}{r}-0.010 \\
(0.069)\end{array}$ & $\begin{array}{r}-0.005 \\
(0.069)\end{array}$ & $\begin{array}{l}-0.154 * \\
(0.092)\end{array}$ & $\begin{array}{l}-0.3633^{* * *} \\
(0.076)\end{array}$ & $\begin{array}{l}-0.363^{* * *} \\
(0.076)\end{array}$ & $\begin{array}{r}-0.173 \\
(0.118)\end{array}$ \\
\hline$($ delta s)* & tariff levels & & & & $\begin{array}{r}-0.029 \\
(0.021)\end{array}$ & $\begin{array}{r}-0.030 \\
(0.022)\end{array}$ & $\begin{array}{l}-0.093 * * \\
(0.039)\end{array}$ & $\begin{array}{l}-0.288^{* * *} \\
(0.069)\end{array}$ & $\begin{array}{l}-0.288^{* * *} \\
(0.069)\end{array}$ & $\begin{array}{r}-0.110 \\
(0.070)\end{array}$ \\
\hline$($ delta s)* & log distance & & & & $\begin{array}{l}-0.079^{* * *} \\
(0.030)\end{array}$ & $\begin{array}{l}-0.079 * * \\
(0.031)\end{array}$ & $\begin{array}{r}-0.116 \\
(0.173)\end{array}$ & $\begin{array}{r}-0.006 \\
(0.137)\end{array}$ & $\begin{array}{r}-0.006 \\
(0.137)\end{array}$ & $\begin{array}{r}-0.148 \\
(0.166)\end{array}$ \\
\hline$($ delta s)* & $\log [$ RGDP(importer)/RGDP(exporter)] & & & & & $\begin{array}{r}0.006 \\
(0.009)\end{array}$ & $\begin{array}{l}0.087 \text { ** } \\
(0.037)\end{array}$ & $\begin{array}{l}-0.2322^{* * *} \\
(0.070)\end{array}$ & $\begin{array}{l}-0.232 * * * \\
(0.070)\end{array}$ & $\begin{array}{c}0.086 \text { ** } \\
(0.034)\end{array}$ \\
\hline$($ delta s)* & log real wage $(\$)$ & & & & & & $\begin{array}{l}-0.0211^{* * *} \\
(0.007)\end{array}$ & $\begin{array}{l}-0.007 * \\
(0.004)\end{array}$ & $\begin{array}{l}-0.007 * \\
(0.004)\end{array}$ & $\begin{array}{l}-0.019 \text { ** } \\
(0.007)\end{array}$ \\
\hline$($ delta s)* & long term inflation & & & & & & & $\begin{array}{l}0.162 \text { *** } \\
(0.051)\end{array}$ & $\begin{array}{l}0.1622^{* * *} \\
(0.051)\end{array}$ & $\begin{array}{r}0.025 \\
(0.046)\end{array}$ \\
\hline$($ delta s)* & long term exchange rate variability & & & & & & & $\begin{array}{r}0.744 \\
(0.505)\end{array}$ & $\begin{array}{r}0.744 \\
(0.505)\end{array}$ & \\
\hline$($ delta s)* & US Importer dummy & & & & & & & & & \\
\hline & Error Correction term (ECM) & $\begin{array}{l}-0.466^{* * *} \\
(0.041)\end{array}$ & $\begin{array}{l}-0.457^{* * *} \\
(0.063)^{*}\end{array}$ & $\begin{array}{l}-0.419^{* * *} \\
(0.080)\end{array}$ & $\begin{array}{r}-0.111 \\
(0.576)\end{array}$ & $\begin{array}{r}-0.029 \\
(0.610)\end{array}$ & $\begin{array}{r}-0.201 \\
(0.664)\end{array}$ & $\begin{array}{r}-0.247 \\
(0.633)\end{array}$ & $\begin{array}{r}-0.247 \\
(0.633)\end{array}$ & $\begin{array}{r}-0.030 \\
(0.674)\end{array}$ \\
\hline $\mathrm{ECM}^{*}$ & trend & & $\begin{array}{c}-0.001 \\
(0.008)\end{array}$ & $\begin{array}{r}-0.002 \\
(0.008)\end{array}$ & $\begin{array}{r}-0.002 \\
(0.008)\end{array}$ & $\begin{array}{r}-0.002 \\
(0.008)\end{array}$ & $\begin{array}{r}0.004 \\
(0.008)\end{array}$ & $\begin{array}{r}0.008 \\
(0.008)\end{array}$ & $\begin{array}{r}0.008 \\
(0.008)\end{array}$ & $\begin{array}{r}0.002 \\
(0.008)\end{array}$ \\
\hline ECM* & $\begin{array}{l}\log [\text { per capitaRGDP(importer) } / \\
\text { per capitaRGDP(exporter)] }\end{array}$ & & & $\begin{array}{r}0.025 \\
(0.034)\end{array}$ & $\begin{array}{r}0.040 \\
(0.049)\end{array}$ & $\begin{array}{r}0.033 \\
(0.049)\end{array}$ & $\begin{array}{r}0.089 \\
(0.074)\end{array}$ & $\begin{array}{r}0.113 \\
(0.078)\end{array}$ & $\begin{array}{r}0.113 \\
(0.078)\end{array}$ & $\begin{array}{r}0.062 \\
(0.077)\end{array}$ \\
\hline $\mathrm{ECM}^{*}$ & tariff levels & & & & $\begin{array}{r}0.023 \\
(0.045)\end{array}$ & $\begin{array}{r}0.017 \\
(0.045)\end{array}$ & $\begin{array}{r}0.051 \\
(0.056)\end{array}$ & $\begin{array}{r}0.043 \\
(0.057)\end{array}$ & $\begin{array}{r}0.043 \\
(0.057)\end{array}$ & $\begin{array}{r}0.047 \\
(0.056)\end{array}$ \\
\hline $\mathrm{ECM}^{*}$ & $\log$ distance & & & & $\begin{array}{r}-0.033 \\
(0.066)\end{array}$ & $\begin{array}{r}-0.036 \\
(0.067)\end{array}$ & $\begin{array}{r}-0.025 \\
(0.075)\end{array}$ & $\begin{array}{r}0.006 \\
(0.073)\end{array}$ & $\begin{array}{r}0.006 \\
(0.073)\end{array}$ & $\begin{array}{r}-0.036 \\
(0.076)\end{array}$ \\
\hline $\mathrm{ECM}^{*}$ & $\log [$ RGDP(importer)/RGDP(exporter)] & & & & & $\begin{array}{r}0.015 \\
(0.029)\end{array}$ & $\begin{array}{r}0.033 \\
(0.037)\end{array}$ & $\begin{array}{l}0.082 * * \\
(0.035)\end{array}$ & $\begin{array}{l}0.082 * * \\
(0.035)\end{array}$ & $\begin{array}{r}0.045 \\
(0.039)\end{array}$ \\
\hline $\mathrm{ECM}^{*}$ & log real wage $(\$)$ & & & & & & $\begin{array}{r}0.023 \\
(0.016)\end{array}$ & $\begin{array}{l}0.025 * \\
(0.015)\end{array}$ & $\begin{array}{l}0.025 * \\
(0.015)\end{array}$ & $\begin{array}{r}0.024 \\
(0.017)\end{array}$ \\
\hline $\mathrm{ECM}^{*}$ & long term inflation & & & & & & & $\begin{array}{r}0.039 \\
(0.119)\end{array}$ & $\begin{array}{r}0.039 \\
(0.119)\end{array}$ & $\begin{array}{r}-0.172 \\
(0.130)\end{array}$ \\
\hline $\mathrm{ECM}^{*}$ & long term exchange rate variability & & & & & & & $\begin{array}{l}-2.617^{* * *} \\
(0.821)\end{array}$ & $\begin{array}{l}-2.617^{* * *} \\
(0.821)\end{array}$ & \\
\hline
\end{tabular}

$\mathrm{ECM}^{*}$ US Importer dummy

\begin{tabular}{|c|c|c|c|c|c|c|c|c|c|}
\hline \# of Observations & 775 & 775 & 775 & 629 & 629 & 384 & 376 & 376 & 384 \\
\hline Adjusted R-squared & 0.946 & 0.945 & 0.945 & 0.950 & 0.950 & 0.962 & 0.960 & 0.960 & 0.962 \\
\hline Country dummies & yes & yes & yes & yes & yes & yes & yes & yes & yes \\
\hline Product dummies & yes & yes & yes & yes & yes & yes & yes & yes & yes \\
\hline
\end{tabular}

Full sample

Product dummies

$*=10 \%, * *=5 \%$, and $* * *=1 \%$ levels of significance

product specific (ps) tariffs 
Table a3: Pass-through to Import price of Cognac

\begin{tabular}{|c|c|c|c|c|c|c|c|c|c|c|}
\hline change in & exchange rate & $\begin{array}{r}\text { Eqn 1 } \\
0.000 \\
(0.000)\end{array}$ & $\begin{array}{r}\text { Eqn2 } \\
0.000 \\
(0.000)\end{array}$ & $\begin{array}{r}\text { Eqn3 } \\
0.000 \\
(0.000)\end{array}$ & $\begin{array}{c}\text { Eqn } 4 \\
0.000 * \\
(0.000)\end{array}$ & $\begin{array}{c}\text { Eqn 5 } \\
0.000 * * \\
(0.000)\end{array}$ & $\begin{array}{c}\text { Eqn 6 } \\
0.000 * \\
(0.000)\end{array}$ & $\begin{array}{c}\text { Eqn } 7 \\
0.000 * \\
(0.000)\end{array}$ & $\begin{array}{c}\text { Eqn 8 } \\
0.000 * \\
(0.000)\end{array}$ & $\begin{array}{r}\text { Eqn } 9 \\
0.000 \\
(0.000)\end{array}$ \\
\hline change in & exporter's price & $\begin{array}{r}0.210 \\
(0.170)\end{array}$ & $\begin{array}{l}0.542 * * * \\
(0.097)\end{array}$ & $\begin{array}{l}0.542 * * * \\
(0.099)\end{array}$ & $\begin{array}{l}0.464 \text { *** } \\
(0.071)\end{array}$ & $\begin{array}{l}0.436 * * * \\
(0.069)\end{array}$ & $\begin{array}{l}0.417^{* * *} \\
(0.089)\end{array}$ & $\begin{array}{l}0.403^{* * *} \\
(0.086)\end{array}$ & $\begin{array}{l}0.401 * * * \\
(0.086)\end{array}$ & $\begin{array}{l}0.405^{* * *} \\
(0.089)\end{array}$ \\
\hline$($ delta s)* & trend & & $\begin{array}{l}0.123^{* * *} \\
(0.016)\end{array}$ & $\begin{array}{l}0.123^{* * *} \\
(0.021)\end{array}$ & $\begin{array}{l}-0.005 \\
(0.012)\end{array}$ & $\begin{array}{r}-0.006 \\
(0.006)\end{array}$ & $\begin{array}{l}-0.054^{* * *} \\
(0.018)\end{array}$ & $\begin{array}{l}-0.067 * * \\
(0.031)\end{array}$ & $\begin{array}{l}-0.068 * * \\
(0.031)\end{array}$ & $\begin{array}{l}-0.045 * * \\
(0.018)\end{array}$ \\
\hline$($ delta s)* & $\begin{array}{l}\log [\text { per capitaRGDP(importer) / } \\
\text { per capitaRGDP(exporter)] }\end{array}$ & & & $\begin{array}{r}-0.009 \\
(0.042)\end{array}$ & $\begin{array}{r}-0.005 \\
(0.011)\end{array}$ & $\begin{array}{l}-0.0611^{* * *} \\
(0.018)\end{array}$ & $\begin{array}{l}-0.137^{* * *} \\
(0.043)\end{array}$ & $\begin{array}{r}0.064 \\
(0.051)\end{array}$ & $\begin{array}{r}0.058 \\
(0.052)\end{array}$ & $\begin{array}{l}-0.115^{* *} \\
(0.047)\end{array}$ \\
\hline$($ delta s)* & tariff levels & & & & $\begin{array}{l}-0.229 * * \\
(0.106)\end{array}$ & $\begin{array}{l}-0.338 \text { *** } \\
(0.068)\end{array}$ & $\begin{array}{l}-0.505^{* * *} \\
(0.077)\end{array}$ & $\begin{array}{l}-0.594^{* * *} \\
(0.081)\end{array}$ & $\begin{array}{l}-0.594^{* * *} \\
(0.081)\end{array}$ & $\begin{array}{l}-0.544^{* * *} \\
(0.075)\end{array}$ \\
\hline$($ delta s)* & log distance & & & & $\begin{array}{l}0.167 \text { *** } \\
(0.029)\end{array}$ & $\begin{array}{l}0.198^{* * *} \\
(0.017)\end{array}$ & $\begin{array}{l}0.278^{* * *} \\
(0.028)\end{array}$ & $\begin{array}{l}0.276 \text { *** } \\
(0.035)\end{array}$ & $\begin{array}{l}0.277^{* * *} \\
(0.035)\end{array}$ & $\begin{array}{l}0.272^{* * *} \\
(0.028)\end{array}$ \\
\hline$($ delta s)* & $\log [$ RGDP(importer)/RGDP(exporter)] & & & & & $\begin{array}{l}0.074^{* * *} \\
(0.019)\end{array}$ & $\begin{array}{c}0.082 \text { ** } \\
(0.042)\end{array}$ & $\begin{array}{l}-0.149^{* * *} \\
(0.056)\end{array}$ & $\begin{array}{l}-0.141 * * \\
(0.059)\end{array}$ & $\begin{array}{r}0.054 \\
(0.046)\end{array}$ \\
\hline$($ delta s)* & log real wage $(\$)$ & & & & & & $\begin{array}{l}-0.055^{* * *} \\
(0.019)\end{array}$ & $\begin{array}{l}-0.069^{* * *} \\
(0.016)\end{array}$ & $\begin{array}{l}-0.067^{* * *} \\
(0.016)\end{array}$ & $\begin{array}{l}-0.056^{* * *} \\
(0.017)\end{array}$ \\
\hline$($ delta s)* & long term inflation & & & & & & & $\begin{array}{l}0.191 \text { *** } \\
(0.068)\end{array}$ & $\begin{array}{l}0.184^{* * *} \\
(0.069)\end{array}$ & $\begin{array}{c}0.059 * \\
(0.035)\end{array}$ \\
\hline$($ delta s)* & long term exchange rate variability & & & & & & & $\begin{array}{r}0.000 \\
(0.000)\end{array}$ & $\begin{array}{r}0.000 \\
(0.000)\end{array}$ & \\
\hline$($ delta s)* & US Importer dummy & & & & & & & & $\begin{array}{r}-0.369 \\
(0.228)\end{array}$ & $\begin{array}{l}-0.950 * * * \\
(0.196)\end{array}$ \\
\hline & Error Correction term (ECM) & $\begin{array}{r}-0.380 \\
(0.248)\end{array}$ & $\begin{array}{r}-0.243 \\
(0.158)\end{array}$ & $\begin{array}{r}-0.114 \\
(0.228)\end{array}$ & $\begin{array}{l}-0.856 * * \\
(0.340)\end{array}$ & $\begin{array}{l}-0.767 * * \\
(0.359)\end{array}$ & $\begin{array}{l}-1.312^{* * *} \\
(0.345)\end{array}$ & $\begin{array}{l}-1.1322^{* * *} \\
(0.383)\end{array}$ & $\begin{array}{l}-1.128^{* * *} \\
(0.385)\end{array}$ & $\begin{array}{l}-1.345^{* * *} \\
(0.340)\end{array}$ \\
\hline ECM* & trend & & $\begin{array}{r}-0.004 \\
(0.009)\end{array}$ & $\begin{array}{r}-0.006 \\
(0.011)\end{array}$ & $\begin{array}{r}0.003 \\
(0.006)\end{array}$ & $\begin{array}{r}0.001 \\
(0.007)\end{array}$ & $\begin{array}{r}0.001 \\
(0.007)\end{array}$ & $\begin{array}{r}0.002 \\
(0.007)\end{array}$ & $\begin{array}{r}0.002 \\
(0.007)\end{array}$ & $\begin{array}{r}0.001 \\
(0.007)\end{array}$ \\
\hline $\mathrm{ECM}^{*}$ & $\begin{array}{l}\log [\text { per capitaRGDP(importer) } / \\
\text { per capitaRGDP(exporter)] }\end{array}$ & & & $\begin{array}{r}0.046 \\
(0.031)\end{array}$ & $\begin{array}{l}0.023 * \\
(0.012)\end{array}$ & $\begin{array}{r}0.029 \\
(0.036)\end{array}$ & $\begin{array}{l}0.088 * * \\
(0.044)\end{array}$ & $\begin{array}{r}0.033 \\
(0.047)\end{array}$ & $\begin{array}{r}0.034 \\
(0.050)\end{array}$ & $\begin{array}{l}0.097^{* *} \\
(0.045)\end{array}$ \\
\hline ECM* & tariff levels & & & & $\begin{array}{l}0.137^{* * *} \\
(0.040)\end{array}$ & $\begin{array}{l}0.134 * * * \\
(0.041)\end{array}$ & $\begin{array}{r}0.082 \\
(0.056)\end{array}$ & $\begin{array}{r}0.068 \\
(0.055)\end{array}$ & $\begin{array}{r}0.068 \\
(0.057)\end{array}$ & $\begin{array}{l}0.096 * \\
(0.056)\end{array}$ \\
\hline $\mathrm{ECM}^{*}$ & log distance & & & & $\begin{array}{r}0.024 \\
(0.040)\end{array}$ & $\begin{array}{r}0.017 \\
(0.040)\end{array}$ & $\begin{array}{l}0.092 * * \\
(0.039)\end{array}$ & $\begin{array}{r}0.078 * \\
(0.041)\end{array}$ & $\begin{array}{r}0.078 * \\
(0.041)\end{array}$ & $\begin{array}{l}0.092 * * \\
(0.039)\end{array}$ \\
\hline ECM* & $\log [$ RGDP(importer)/RGDP(exporter)] & & & & & $\begin{array}{r}-0.004 \\
(0.034)\end{array}$ & $\begin{array}{r}-0.045 \\
(0.041)\end{array}$ & $\begin{array}{r}0.006 \\
(0.042)\end{array}$ & $\begin{array}{r}0.004 \\
(0.047)\end{array}$ & $\begin{array}{r}-0.054 \\
(0.043)\end{array}$ \\
\hline ECM* & log real wage $(\$)$ & & & & & & $\begin{array}{r}0.019 \\
(0.016)\end{array}$ & $\begin{array}{r}0.018 \\
(0.016)\end{array}$ & $\begin{array}{r}0.018 \\
(0.016)\end{array}$ & $\begin{array}{r}0.018 \\
(0.015)\end{array}$ \\
\hline ECM* & long term inflation & & & & & & & $\begin{array}{r}0.002 \\
(0.061)\end{array}$ & $\begin{array}{r}0.002 \\
(0.061)\end{array}$ & $\begin{array}{r}0.012 \\
(0.067)\end{array}$ \\
\hline ECM* & long term exchange rate variability & & & & & & & $\begin{array}{r}0.412 \\
(0.256)\end{array}$ & $\begin{array}{r}0.399 \\
(0.255)\end{array}$ & \\
\hline $\mathrm{ECM}^{*}$ & US Importer dummy & & & & & & & & $\begin{array}{r}0.017 \\
(0.190)\end{array}$ & $\begin{array}{r}0.155 \\
(0.191)\end{array}$ \\
\hline & \# of Observations & 669 & 669 & 669 & 569 & 569 & 384 & 376 & 376 & 384 \\
\hline & Adjusted R-squared & -0.011 & 0.750 & 0.753 & 0.945 & 0.950 & 0.955 & 0.952 & 0.951 & 0.956 \\
\hline & Country dummies & yes & yes & yes & yes & yes & yes & yes & yes & yes \\
\hline & Product dummies & yes & yes & yes & yes & yes & yes & yes & yes & yes \\
\hline
\end{tabular}

Full sample

$*=10 \%, * *=5 \%$, and $* * *=1 \%$ levels of significance

product specific (ps) tariffs 
Table a4: Pass-through to Import price of Gilbey's Gin

\begin{tabular}{|c|c|c|c|c|c|c|c|c|c|c|}
\hline change in & exchange rate & $\begin{array}{c}\text { Eqn 1 } \\
0.957 * * * \\
(0.020)\end{array}$ & $\begin{array}{c}\text { Eqn2 } \\
0.876 * * * \\
(0.030)\end{array}$ & $\begin{array}{c}\text { Eqn3 } \\
0.839 \\
(0.064)\end{array}$ & $\begin{array}{c}\text { Eqn } 4 \\
0.852 * * * \\
(0.243)\end{array}$ & $\begin{array}{c}\text { Eqn 5 } \\
0.8277^{* * *} \\
(0.250)\end{array}$ & $\begin{array}{r}\text { Eqn 6 } \\
0.770 \\
(1.160)\end{array}$ & $\begin{array}{r}\text { Eqn } 7 \\
-1.795 \\
(1.800)\end{array}$ & $\begin{array}{r}\text { Eqn } 8 \\
-1.795 \\
(1.800)\end{array}$ & $\begin{array}{r}\text { Eqn 9 } \\
0.563 \\
(1.241)\end{array}$ \\
\hline change in & exporter's price & $\begin{array}{l}0.7411^{* * *} \\
(0.109)\end{array}$ & $\begin{array}{l}0.644 \text { *** } \\
(0.116)\end{array}$ & $\begin{array}{l}0.5933^{* * *} \\
(0.103)\end{array}$ & $\begin{array}{l}0.427^{* * *} \\
(0.103)\end{array}$ & $\begin{array}{l}0.420 \text { *** } \\
(0.101)\end{array}$ & $\begin{array}{c}0.232 * \\
(0.134)\end{array}$ & $\begin{array}{l}0.316^{* * *} \\
(0.109)\end{array}$ & $\begin{array}{l}0.316 \text { *** } \\
(0.109)\end{array}$ & $\begin{array}{c}0.258 \text { ** } \\
(0.132)\end{array}$ \\
\hline$($ delta s)* & trend & & $\begin{array}{l}0.013 \text { *** } \\
(0.004)\end{array}$ & $\begin{array}{r}0.010 \\
(0.010)\end{array}$ & $\begin{array}{r}-0.001 \\
(0.015)\end{array}$ & $\begin{array}{r}-0.013 \\
(0.014)\end{array}$ & $\begin{array}{l}-0.0388^{* * *} \\
(0.010)\end{array}$ & $\begin{array}{r}-0.043 \\
(0.031)\end{array}$ & $\begin{array}{r}-0.043 \\
(0.031)\end{array}$ & $\begin{array}{l}-0.026^{* * *} \\
(0.009)\end{array}$ \\
\hline$($ delta s)* & $\begin{array}{l}\log [\text { per capitaRGDP(importer) / } \\
\text { per capitaRGDP(exporter)] }\end{array}$ & & & $\begin{array}{r}-0.039 \\
(0.076)\end{array}$ & $\begin{array}{l}-0.186 * * * \\
(0.072)\end{array}$ & $\begin{array}{l}-0.159 * * \\
(0.072)\end{array}$ & $\begin{array}{l}-0.585^{* * *} \\
(0.073)\end{array}$ & $\begin{array}{l}-0.532 * * * \\
(0.093)\end{array}$ & $\begin{array}{l}-0.532 * * * \\
(0.093)\end{array}$ & $\begin{array}{l}-0.494^{* * *} \\
(0.073)\end{array}$ \\
\hline$($ delta s)* & tariff levels & & & & $\begin{array}{l}-0.081 * * \\
(0.034)\end{array}$ & $\begin{array}{l}-0.135 \text { *** } \\
(0.038)\end{array}$ & $\begin{array}{l}-0.3233^{* * *} \\
(0.051)\end{array}$ & $\begin{array}{l}-0.300 * * * \\
(0.087)\end{array}$ & $\begin{array}{l}-0.300 * * * \\
(0.087)\end{array}$ & $\begin{array}{l}-0.397^{* * *} \\
(0.067)^{*}\end{array}$ \\
\hline$($ delta s)* & $\log$ distance & & & & $\begin{array}{r}-0.002 \\
(0.024)\end{array}$ & $\begin{array}{r}0.038 \\
(0.031)\end{array}$ & $\begin{array}{r}0.062 \\
(0.137)\end{array}$ & $\begin{array}{r}0.288 \\
(0.207)\end{array}$ & $\begin{array}{r}0.288 \\
(0.207)\end{array}$ & $\begin{array}{r}0.091 \\
(0.151)\end{array}$ \\
\hline$($ delta s)* & $\log [$ RGDP(importer)/RGDP(exporter)] & & & & & $\begin{array}{l}0.045^{* * *} \\
(0.016)\end{array}$ & $\begin{array}{l}0.169 \text { *** } \\
(0.029)\end{array}$ & $\begin{array}{r}-0.028 \\
(0.081)\end{array}$ & $\begin{array}{r}-0.028 \\
(0.081)\end{array}$ & $\begin{array}{l}0.148^{* * *} \\
(0.029)\end{array}$ \\
\hline$($ delta s)* & log real wage (\$) & & & & & & $\begin{array}{r}-0.001 \\
(0.007)\end{array}$ & $\begin{array}{r}0.001 \\
(0.007)\end{array}$ & $\begin{array}{r}0.001 \\
(0.007)\end{array}$ & $\begin{array}{r}0.005 \\
(0.007)\end{array}$ \\
\hline$($ delta s)* & long term inflation & & & & & & & $\begin{array}{l}0.175 * \\
(0.102)\end{array}$ & $\begin{array}{l}0.175 * \\
(0.102)\end{array}$ & $\begin{array}{c}0.054 * \\
(0.029)\end{array}$ \\
\hline$($ delta s)* & long term exchange rate variability & & & & & & & $\begin{array}{r}-2.101 \\
(1.598)\end{array}$ & $\begin{array}{r}-2.101 \\
(1.598)\end{array}$ & \\
\hline$($ delta s)* & US Importer dummy & & & & & & & & & \\
\hline & Error Correction term (ECM) & $\begin{array}{l}-0.459 \text { *** } \\
(0.062)\end{array}$ & $\begin{array}{l}-0.401 * * * \\
(0.063)\end{array}$ & $\begin{array}{l}-0.1588^{* *} \\
(0.080)\end{array}$ & $\begin{array}{r}-0.303 \\
(0.738)\end{array}$ & $\begin{array}{c}-0.449 \\
(0.710)\end{array}$ & $\begin{array}{r}-0.021 \\
(0.707)\end{array}$ & $\begin{array}{r}0.460 \\
(0.569)\end{array}$ & $\begin{array}{r}0.460 \\
(0.569)\end{array}$ & $\begin{array}{r}0.198 \\
(0.700)\end{array}$ \\
\hline $\mathrm{ECM}^{*}$ & trend & & $\begin{array}{l}-0.014 \text { *** } \\
(0.004)\end{array}$ & $\begin{array}{l}-0.012^{* * *} \\
(0.004)\end{array}$ & $\begin{array}{l}-0.010 \text { ** } \\
(0.005)\end{array}$ & $\begin{array}{l}-0.008 * \\
(0.005)\end{array}$ & $\begin{array}{l}-0.010 * \\
(0.005)\end{array}$ & $\begin{array}{l}-0.014^{* * *} \\
(0.005)\end{array}$ & $\begin{array}{l}-0.0144^{* * *} \\
(0.005)\end{array}$ & $\begin{array}{l}-0.014^{* * *} \\
(0.005)\end{array}$ \\
\hline ECM* & $\begin{array}{l}\log [\text { per capitaRGDP(importer) / } \\
\text { per capitaRGDP(exporter)] }\end{array}$ & & & $\begin{array}{l}0.161 * * \\
(0.071)\end{array}$ & $\begin{array}{l}0.184^{* * *} \\
(0.053)\end{array}$ & $\begin{array}{l}0.175^{* * *} \\
(0.056)\end{array}$ & $\begin{array}{l}0.285 * * * \\
(0.079)\end{array}$ & $\begin{array}{l}0.146 * \\
(0.076)\end{array}$ & $\begin{array}{l}0.146 * \\
(0.076)\end{array}$ & $\begin{array}{l}0.188^{* * *} \\
(0.072)\end{array}$ \\
\hline $\mathrm{ECM}^{*}$ & tariff levels & & & & $\begin{array}{l}0.068^{* *} \\
(0.026)\end{array}$ & $\begin{array}{l}0.057 * * \\
(0.027)\end{array}$ & $\begin{array}{r}0.002 \\
(0.029)\end{array}$ & $\begin{array}{r}0.001 \\
(0.031)\end{array}$ & $\begin{array}{r}0.001 \\
(0.031)\end{array}$ & $\begin{array}{r}0.006 \\
(0.028)\end{array}$ \\
\hline $\mathrm{ECM}^{*}$ & $\log$ distance & & & & $\begin{array}{r}0.006 \\
(0.085)\end{array}$ & $\begin{array}{r}0.020 \\
(0.082)\end{array}$ & $\begin{array}{r}-0.013 \\
(0.087)\end{array}$ & $\begin{array}{r}-0.071 \\
(0.072)\end{array}$ & $\begin{array}{r}-0.071 \\
(0.072)\end{array}$ & $\begin{array}{l}-0.043 \\
(0.088)\end{array}$ \\
\hline $\mathrm{ECM}^{*}$ & $\log [$ RGDP(importer)/RGDP(exporter)] & & & & & $\begin{array}{r}-0.014 \\
(0.032)\end{array}$ & $\begin{array}{r}-0.023 \\
(0.047)\end{array}$ & $\begin{array}{r}0.007 \\
(0.044)\end{array}$ & $\begin{array}{r}0.007 \\
(0.044)\end{array}$ & $\begin{array}{r}-0.015 \\
(0.043)\end{array}$ \\
\hline $\mathrm{ECM}^{*}$ & log real wage $(\$)$ & & & & & & $\begin{array}{r}0.005 \\
(0.009)\end{array}$ & $\begin{array}{r}0.015 \\
(0.015)\end{array}$ & $\begin{array}{r}0.015 \\
(0.015)\end{array}$ & $\begin{array}{r}0.009 \\
(0.010)\end{array}$ \\
\hline $\mathrm{ECM}^{*}$ & long term inflation & & & & & & & $\begin{array}{r}-0.123 \\
(0.088)\end{array}$ & $\begin{array}{r}-0.123 \\
(0.088)\end{array}$ & $\begin{array}{l}-0.110 * \\
(0.062)\end{array}$ \\
\hline ECM* & long term exchange rate variability & & & & & & & $\begin{array}{r}-0.714 \\
(1.682)\end{array}$ & $\begin{array}{r}-0.714 \\
(1.682)\end{array}$ & \\
\hline \multicolumn{11}{|c|}{ ECM* US Importer dummy } \\
\hline & \# of Observations & 729 & 729 & 729 & 587 & 587 & 382 & 374 & 374 & 382 \\
\hline & Adjusted R-squared & 0.924 & 0.926 & 0.929 & 0.943 & 0.945 & 0.962 & 0.951 & 0.951 & 0.963 \\
\hline & Country dummies & yes & yes & yes & yes & yes & yes & yes & yes & yes \\
\hline & Product dummies & yes & yes & yes & yes & yes & yes & yes & yes & yes \\
\hline
\end{tabular}

Full sample

Product dummies

$*=10 \%, * *=5 \%$, and $* * *=1 \%$ levels of significance

product specific (ps) tariffs 
Table a5: Pass-through to Import price of Time magazine

\begin{tabular}{|c|c|c|c|c|c|c|c|c|c|c|}
\hline change in & exchange rate & $\begin{array}{c}\text { Eqn 1 } \\
0.985 * * * \\
(0.011)\end{array}$ & $\begin{array}{c}\text { Eqn2 } \\
1.011^{* * *} \\
(0.014)\end{array}$ & $\begin{array}{c}\text { Eqn3 } \\
0.932 * * * \\
(0.044)\end{array}$ & $\begin{array}{c}\text { Eqn } 4 \\
1.110^{* * *} \\
(0.225)\end{array}$ & $\begin{array}{c}\text { Eqn 5 } \\
1.101{ }^{* * *} \\
(0.243)\end{array}$ & $\begin{array}{r}\text { Eqn 6 } \\
0.971 \\
(1.128)\end{array}$ & $\begin{array}{c}\text { Eqn } 7 \\
3.078 * * * \\
(1.080)\end{array}$ & $\begin{array}{c}\text { Eqn 8 } \\
3.078^{* * *} \\
(1.080)\end{array}$ & $\begin{array}{r}\text { Eqn 9 } \\
1.102 \\
(1.072)\end{array}$ \\
\hline change in & exporter's price & $\begin{array}{r}0.000 \\
(0.080)\end{array}$ & $\begin{array}{r}0.018 \\
(0.081)\end{array}$ & $\begin{array}{r}0.024 \\
(0.080)\end{array}$ & $\begin{array}{r}0.065 \\
(0.091)\end{array}$ & $\begin{array}{r}0.066 \\
(0.091)\end{array}$ & $\begin{array}{r}-0.014 \\
(0.096)\end{array}$ & $\begin{array}{r}-0.050 \\
(0.090)\end{array}$ & $\begin{array}{r}-0.050 \\
(0.090)\end{array}$ & $\begin{array}{r}-0.011 \\
(0.097)\end{array}$ \\
\hline$($ delta s)* & trend & & $\begin{array}{r}-0.004 \\
(0.003)\end{array}$ & $\begin{array}{l}-0.014^{* *} \\
(0.007)\end{array}$ & $\begin{array}{l}-0.026 * * * \\
(0.010)\end{array}$ & $\begin{array}{l}-0.023 * * \\
(0.011)\end{array}$ & $\begin{array}{l}-0.047^{* * *} \\
(0.018)\end{array}$ & $\begin{array}{l}-0.082 * * * \\
(0.020)\end{array}$ & $\begin{array}{l}-0.082 * * * \\
(0.020)\end{array}$ & $\begin{array}{l}-0.050^{* * *} \\
(0.017)\end{array}$ \\
\hline$($ delta s)* & $\begin{array}{l}\log [\text { per capitaRGDP(importer) / } \\
\text { per capitaRGDP(exporter)] }\end{array}$ & & & $\begin{array}{l}-0.094 * \\
(0.051)\end{array}$ & $\begin{array}{l}-0.156^{* *} \\
(0.071)\end{array}$ & $\begin{array}{l}-0.156 * * \\
(0.070)\end{array}$ & $\begin{array}{l}-0.250 * * \\
(0.097)\end{array}$ & $\begin{array}{l}-0.435 * * * \\
(0.124)\end{array}$ & $\begin{array}{l}-0.435 * * * \\
(0.124)\end{array}$ & $\begin{array}{l}-0.276^{* * *} \\
(0.086)\end{array}$ \\
\hline$($ delta s)* & tariff levels & & & & $\begin{array}{l}-0.075^{* *} \\
(0.031)\end{array}$ & $\begin{array}{l}-0.077^{* *} \\
(0.033)\end{array}$ & $\begin{array}{l}-0.118^{* * *} \\
(0.040)\end{array}$ & $\begin{array}{l}-0.269 * * * \\
(0.061)\end{array}$ & $\begin{array}{l}-0.269 * * * \\
(0.061)\end{array}$ & $\begin{array}{l}-0.093 * \\
(0.052)\end{array}$ \\
\hline$($ delta s)* & log distance & & & & $\begin{array}{r}-0.006 \\
(0.026)\end{array}$ & $\begin{array}{r}-0.001 \\
(0.029)\end{array}$ & $\begin{array}{r}0.042 \\
(0.130)\end{array}$ & $\begin{array}{r}-0.193 \\
(0.123)\end{array}$ & $\begin{array}{r}-0.193 \\
(0.123)\end{array}$ & $\begin{array}{r}0.024 \\
(0.123)\end{array}$ \\
\hline$($ delta s)* & $\log [$ RGDP(importer)/RGDP(exporter)] & & & & & $\begin{array}{r}0.014 \\
(0.013)\end{array}$ & $\begin{array}{r}0.064 \\
(0.041)\end{array}$ & $\begin{array}{r}0.061 \\
(0.069)\end{array}$ & $\begin{array}{r}0.061 \\
(0.069)\end{array}$ & $\begin{array}{c}0.067 * \\
(0.039)\end{array}$ \\
\hline$($ delta s)* & log real wage $(\$)$ & & & & & & $\begin{array}{l}-0.015 * \\
(0.008)\end{array}$ & $\begin{array}{l}-0.007 * \\
(0.004)\end{array}$ & $\begin{array}{l}-0.007 * \\
(0.004)\end{array}$ & $\begin{array}{l}-0.016 * * \\
(0.007)\end{array}$ \\
\hline$($ delta s)* & long term inflation & & & & & & & $\begin{array}{r}-0.003 \\
(0.037)\end{array}$ & $\begin{array}{r}-0.003 \\
(0.037)\end{array}$ & $\begin{array}{r}-0.019 \\
(0.031)\end{array}$ \\
\hline$($ delta s)* & long term exchange rate variability & & & & & & & $\begin{array}{l}1.299 * * \\
(0.573)\end{array}$ & $\begin{array}{l}1.299 * * \\
(0.573)\end{array}$ & \\
\hline$($ delta s)* & US Importer dummy & & & & & & & & & \\
\hline & Error Correction term (ECM) & $\begin{array}{l}-0.314 * * * \\
(0.042)\end{array}$ & $\begin{array}{l}-0.367 * * * \\
(0.075)\end{array}$ & $\begin{array}{l}-0.404^{* * *} \\
(0.081)\end{array}$ & $\begin{array}{l}-1.757 * \\
(0.927)\end{array}$ & $\begin{array}{l}-2.160 * * \\
(0.947)\end{array}$ & $\begin{array}{l}-2.9011^{* * *} \\
(0.825)\end{array}$ & $\begin{array}{l}-2.310 * * * \\
(0.735)\end{array}$ & $\begin{array}{l}-2.310 * * * \\
(0.735)\end{array}$ & $\begin{array}{l}-2.932 \text { *** } \\
(0.800)\end{array}$ \\
\hline ECM* & trend & & $\begin{array}{r}0.006 \\
(0.006)\end{array}$ & $\begin{array}{r}0.005 \\
(0.006)\end{array}$ & $\begin{array}{r}0.004 \\
(0.007)\end{array}$ & $\begin{array}{r}0.006 \\
(0.008)\end{array}$ & $\begin{array}{c}0.020 * \\
(0.012)\end{array}$ & $\begin{array}{r}0.012 \\
(0.012)\end{array}$ & $\begin{array}{r}0.012 \\
(0.012)\end{array}$ & $\begin{array}{c}0.021 * \\
(0.012)\end{array}$ \\
\hline $\mathrm{ECM}^{*}$ & $\begin{array}{l}\log [\text { per capitaRGDP(importer) / } \\
\text { per capitaRGDP(exporter)] }\end{array}$ & & & $\begin{array}{r}-0.026 \\
(0.033)\end{array}$ & $\begin{array}{r}-0.044 \\
(0.042)\end{array}$ & $\begin{array}{r}-0.013 \\
(0.047)\end{array}$ & $\begin{array}{l}0.120 * \\
(0.068)\end{array}$ & $\begin{array}{r}0.061 \\
(0.056)\end{array}$ & $\begin{array}{r}0.061 \\
(0.056)\end{array}$ & $\begin{array}{l}0.128 * \\
(0.069)\end{array}$ \\
\hline $\mathrm{ECM}^{*}$ & tariff levels & & & & $\begin{array}{r}0.032 \\
(0.041)\end{array}$ & $\begin{array}{r}0.037 \\
(0.040)\end{array}$ & $\begin{array}{l}0.1300^{* *} \\
(0.053)\end{array}$ & $\begin{array}{l}0.083 * \\
(0.044)\end{array}$ & $\begin{array}{l}0.083 * \\
(0.044)\end{array}$ & $\begin{array}{l}0.128 * * \\
(0.053)\end{array}$ \\
\hline $\mathrm{ECM}^{*}$ & $\log$ distance & & & & $\begin{array}{r}0.142 \\
(0.106)\end{array}$ & $\begin{array}{r}0.168 \\
(0.105)\end{array}$ & $\begin{array}{l}0.245^{* *} \\
(0.100)\end{array}$ & $\begin{array}{l}0.216^{* *} \\
(0.091)\end{array}$ & $\begin{array}{l}0.216 \text { ** } \\
(0.091)\end{array}$ & $\begin{array}{l}0.249 \text { ** } \\
(0.098)\end{array}$ \\
\hline $\mathrm{ECM}^{*}$ & $\log [$ RGDP(importer)/RGDP(exporter)] & & & & & $\begin{array}{r}-0.047 \\
(0.036)\end{array}$ & $\begin{array}{r}0.012 \\
(0.052)\end{array}$ & $\begin{array}{r}0.038 \\
(0.044)\end{array}$ & $\begin{array}{r}0.038 \\
(0.044)\end{array}$ & $\begin{array}{r}0.010 \\
(0.054)\end{array}$ \\
\hline $\mathrm{ECM}^{*}$ & log real wage $(\$)$ & & & & & & $\begin{array}{r}0.018 \\
(0.014)\end{array}$ & $\begin{array}{r}0.003 \\
(0.015)\end{array}$ & $\begin{array}{r}0.003 \\
(0.015)\end{array}$ & $\begin{array}{r}0.015 \\
(0.014)\end{array}$ \\
\hline $\mathrm{ECM}^{*}$ & long term inflation & & & & & & & $\begin{array}{r}-0.204 \\
(0.148)\end{array}$ & $\begin{array}{r}-0.204 \\
(0.148)\end{array}$ & $\begin{array}{r}0.030 \\
(0.120)\end{array}$ \\
\hline $\mathrm{ECM}^{*}$ & long term exchange rate variability & & & & & & & $\begin{array}{r}-1.418 \\
(2.579)\end{array}$ & $\begin{array}{r}-1.418 \\
(2.579)\end{array}$ & \\
\hline
\end{tabular}

ECM* US Importer dummy

\begin{tabular}{|c|c|c|c|c|c|c|c|c|c|}
\hline \# of Observations & 766 & 766 & 766 & 625 & 625 & 386 & 377 & 377 & 386 \\
\hline Adjusted R-squared & 0.961 & 0.961 & 0.962 & 0.964 & 0.964 & 0.975 & 0.976 & 0.976 & 0.975 \\
\hline Country dummies & yes & yes & yes & yes & yes & yes & yes & yes & yes \\
\hline Product dummies & yes & yes & yes & yes & yes & yes & yes & yes & yes \\
\hline
\end{tabular}

Full sample

$*=10 \%, * *=5 \%$, and $* * *=1 \%$ levels of significance

product specific $(\mathrm{ps})$ tariffs 
Table a6: Pass-through to Import price of Kodak film

\begin{tabular}{|c|c|}
\hline change in & exchange rate \\
\hline change in & exporter's price \\
\hline$(\text { delta } \mathrm{s})^{*}$ & trend \\
\hline$(\text { delta } s)^{*}$ & $\begin{array}{l}\log [\text { per capitaRGDP(importer) / } \\
\text { per capitaRGDP(exporter)] }\end{array}$ \\
\hline$($ delta s)* & tariff levels \\
\hline$(\text { delta } s)^{*}$ & log distance \\
\hline$(\text { delta } \mathrm{s})^{*}$ & $\log [$ RGDP(importer)/RGDP(exporter)] \\
\hline$(\text { delta } s)^{*}$ & log real wage $(\$)$ \\
\hline$($ delta s)* & long term inflation \\
\hline$($ delta s)* & long term exchange rate variability \\
\hline$($ delta s)* & US Importer dummy \\
\hline
\end{tabular}

\begin{tabular}{|c|c|c|c|c|c|c|c|c|}
\hline $\begin{array}{l}\text { Eqn 1 } \\
1.001 * * * \\
(0.009)\end{array}$ & $\begin{array}{c}\text { Eqn2 } \\
0.996 * * * \\
(0.013)\end{array}$ & $\begin{array}{c}\text { Eqn3 } \\
0.9866^{* * *} \\
(0.033)\end{array}$ & $\begin{array}{l}\text { Eqn 4 } \\
1.2844^{* * *} \\
(0.285)\end{array}$ & $\begin{array}{c}\text { Eqn 5 } \\
1.2699^{* * *} \\
(0.304)\end{array}$ & $\begin{array}{c}\text { Eqn 6 } \\
4.530 * * * \\
(1.696)\end{array}$ & $\begin{array}{l}\text { Eqn } 7 \\
5.6644^{* * *} \\
(1.728)\end{array}$ & $\begin{array}{c}\text { Eqn 8 } \\
5.6644^{* * *} \\
(1.728)\end{array}$ & $\begin{array}{c}\text { Eqn 9 } \\
4.5355^{* * *} \\
(1.607)\end{array}$ \\
\hline \multirow[t]{9}{*}{$\begin{array}{l}-0.551 \text { *** } \\
(0.129)\end{array}$} & $\begin{array}{l}-0.321{ }^{* *} \\
(0.131)\end{array}$ & $\begin{array}{l}-0.313 \text { ** } \\
(0.137)\end{array}$ & $\begin{array}{l}-0.355 * * \\
(0.164)\end{array}$ & $\begin{array}{l}-0.375 * * \\
(0.152)\end{array}$ & $\begin{array}{l}-0.291 * \\
(0.170)\end{array}$ & $\begin{array}{r}0.001 \\
(0.126)\end{array}$ & $\begin{array}{r}0.001 \\
(0.126)\end{array}$ & $\begin{array}{l}-0.288 * \\
(0.165)\end{array}$ \\
\hline & $\begin{array}{r}0.001 \\
(0.003)\end{array}$ & $\begin{array}{l}-0.001 \\
(0.005)\end{array}$ & $\begin{array}{r}-0.004 \\
(0.007)\end{array}$ & $\begin{array}{r}-0.002 \\
(0.008)\end{array}$ & $\begin{array}{l}-0.038 * * \\
(0.017)\end{array}$ & $\begin{array}{l}-0.089 * * * \\
(0.019)\end{array}$ & $\begin{array}{l}-0.089 * * * \\
(0.019)\end{array}$ & $\begin{array}{l}-0.038 * * \\
(0.015)\end{array}$ \\
\hline & & $\begin{array}{r}-0.012 \\
(0.036)\end{array}$ & $\begin{array}{l}-0.041 \\
(0.057)\end{array}$ & $\begin{array}{r}-0.040 \\
(0.055)\end{array}$ & $\begin{array}{l}-0.185 * \\
(0.099)\end{array}$ & $\begin{array}{l}-0.380 * * * \\
(0.095)\end{array}$ & $\begin{array}{l}-0.380 * * * \\
(0.095)\end{array}$ & $\begin{array}{l}-0.187 * \\
(0.097)\end{array}$ \\
\hline & & & $\begin{array}{l}-0.007 \\
(0.020)\end{array}$ & $\begin{array}{l}-0.007 \\
(0.022)\end{array}$ & $\begin{array}{l}-0.027 \\
(0.045)\end{array}$ & $\begin{array}{l}-0.127 * \\
(0.073)\end{array}$ & $\begin{array}{l}-0.127 * \\
(0.073)\end{array}$ & $\begin{array}{l}-0.029 \\
(0.067)\end{array}$ \\
\hline & & & $\begin{array}{c}-0.034 \\
(0.030)\end{array}$ & $\begin{array}{r}-0.030 \\
(0.032)\end{array}$ & $\begin{array}{l}-0.356 * \\
(0.193)\end{array}$ & $\begin{array}{l}-0.489 * * \\
(0.216)\end{array}$ & $\begin{array}{l}-0.489 * * \\
(0.216)\end{array}$ & $\begin{array}{l}-0.356 * \\
(0.184)\end{array}$ \\
\hline & & & & $\begin{array}{r}0.011 \\
(0.011)\end{array}$ & $\begin{array}{l}0.099 \text { ** } \\
(0.042)\end{array}$ & $\begin{array}{r}0.051 \\
(0.082)\end{array}$ & $\begin{array}{r}0.051 \\
(0.082)\end{array}$ & $\begin{array}{l}0.1011^{* * *} \\
(0.039)\end{array}$ \\
\hline & & & & & $\begin{array}{l}-0.018 * * \\
(0.009)\end{array}$ & $\begin{array}{l}-0.015 * * * \\
(0.006)\end{array}$ & $\begin{array}{l}-0.015 * * * \\
(0.006)\end{array}$ & $\begin{array}{l}-0.018 * * \\
(0.008)\end{array}$ \\
\hline & & & & & & $\begin{array}{r}0.002 \\
(0.043)\end{array}$ & $\begin{array}{r}0.002 \\
(0.043)\end{array}$ & $\begin{array}{r}0.001 \\
(0.035)\end{array}$ \\
\hline & & & & & & $\begin{array}{r}0.412 \\
(0.691)\end{array}$ & $\begin{array}{r}0.412 \\
(0.691)\end{array}$ & \\
\hline
\end{tabular}

(delta s) * US Importer dumny

\section{Error Correction term (ECM)}

$\mathrm{ECM}^{*}$ trend

$\mathrm{ECM}^{*} \log [$ per capitaRGDP(importer) $/$
per capitaRGDP(exporter)]

ECM* tariff levels

$\mathrm{ECM}^{*} \log$ distance

ECM* $\log [$ RGDP(importer)/RGDP(exporter)]

$\mathrm{ECM}^{*} \log$ real wage (\$)

$\mathrm{ECM}^{*}$ long term inflation

$\mathrm{ECM}^{*}$ long term exchange rate variability

ECM* US Importer dummy

\begin{tabular}{|c|c|c|c|c|c|c|c|c|}
\hline \multirow[t]{9}{*}{$\begin{array}{l}-0.250^{* * *} \\
(0.035)\end{array}$} & $\begin{array}{l}-0.520 * * * \\
(0.076)\end{array}$ & $\begin{array}{l}-0.492 * * * \\
(0.071)\end{array}$ & $\begin{array}{l}-1.2611^{* *} \\
(0.585)\end{array}$ & $\begin{array}{l}-1.5300^{* *} \\
(0.613)\end{array}$ & $\begin{array}{r}-0.956 \\
(0.660)\end{array}$ & $\begin{array}{l}-1.5422^{* *} \\
(0.618)\end{array}$ & $\begin{array}{l}-1.5422^{* *} \\
(0.618)\end{array}$ & $\begin{array}{r}-0.888 \\
(0.677)\end{array}$ \\
\hline & $\begin{array}{l}0.029 * * * \\
(0.006)\end{array}$ & $\begin{array}{l}0.030 \text { *** } \\
(0.006)\end{array}$ & $\begin{array}{l}0.027 \text { *** } \\
(0.007)\end{array}$ & $\begin{array}{l}0.027^{* * *} \\
(0.007)\end{array}$ & $\begin{array}{l}0.029 \text { *** } \\
(0.008)\end{array}$ & $\begin{array}{l}0.025^{* * *} \\
(0.008)\end{array}$ & $\begin{array}{l}0.025^{* * *} \\
(0.008)\end{array}$ & $\begin{array}{l}0.0288^{* * *} \\
(0.008)\end{array}$ \\
\hline & & $\begin{array}{r}0.024 \\
(0.034)\end{array}$ & $\begin{array}{r}0.057 \\
(0.037)\end{array}$ & $\begin{array}{c}0.080 * \\
(0.044)\end{array}$ & $\begin{array}{r}0.011 \\
(0.047)\end{array}$ & $\begin{array}{r}0.067 \\
(0.049)\end{array}$ & $\begin{array}{r}0.067 \\
(0.049)\end{array}$ & $\begin{array}{r}0.003 \\
(0.052)\end{array}$ \\
\hline & & & $\begin{array}{r}-0.015 \\
(0.031)\end{array}$ & $\begin{array}{r}-0.003 \\
(0.026)\end{array}$ & $\begin{array}{r}-0.008 \\
(0.039)\end{array}$ & $\begin{array}{r}0.014 \\
(0.040)\end{array}$ & $\begin{array}{r}0.014 \\
(0.040)\end{array}$ & $\begin{array}{r}-0.011 \\
(0.039)\end{array}$ \\
\hline & & & $\begin{array}{r}0.096 \\
(0.067)\end{array}$ & $\begin{array}{r}0.109 \\
(0.068)\end{array}$ & $\begin{array}{r}0.019 \\
(0.078)\end{array}$ & $\begin{array}{r}0.093 \\
(0.073)\end{array}$ & $\begin{array}{r}0.093 \\
(0.073)\end{array}$ & $\begin{array}{r}0.013 \\
(0.079)\end{array}$ \\
\hline & & & & $\begin{array}{r}-0.042 \\
(0.029)\end{array}$ & $\begin{array}{l}-0.074^{* *} \\
(0.033)\end{array}$ & $\begin{array}{l}-0.059 * \\
(0.033)\end{array}$ & $\begin{array}{l}-0.059 * \\
(0.033)\end{array}$ & $\begin{array}{l}-0.073 \text { ** } \\
(0.034)\end{array}$ \\
\hline & & & & & $\begin{array}{r}0.019 \\
(0.013)\end{array}$ & $\begin{array}{r}0.022 \\
(0.013)\end{array}$ & $\begin{array}{r}0.022 \\
(0.013)\end{array}$ & $\begin{array}{r}0.020 \\
(0.013)\end{array}$ \\
\hline & & & & & & $\begin{array}{r}0.037 \\
(0.158)\end{array}$ & $\begin{array}{r}0.037 \\
(0.158)\end{array}$ & $\begin{array}{r}-0.047 \\
(0.095)\end{array}$ \\
\hline & & & & & & $\begin{array}{r}0.085 \\
(1.270)\end{array}$ & $\begin{array}{r}0.085 \\
(1.270)\end{array}$ & \\
\hline
\end{tabular}

\begin{tabular}{|c|c|c|c|c|c|c|c|c|c|}
\hline \# of Observations & 782 & 782 & 782 & 636 & 636 & 387 & 378 & 378 & 387 \\
\hline Adjusted R-squared & 0.968 & 0.970 & 0.970 & 0.971 & 0.971 & 0.977 & 0.977 & 0.977 & 0.976 \\
\hline Country dummies & yes & yes & yes & yes & yes & yes & yes & yes & yes \\
\hline Product dummies & yes & yes & yes & yes & yes & yes & yes & yes & yes \\
\hline
\end{tabular}

Full sample

$*=10 \%, * *=5 \%$, and $* * *=1 \%$ levels of significance

product specific (ps) tariffs 
Table a7: Pass-through to Import price of Cointreau

\begin{tabular}{|c|c|c|c|c|c|c|c|c|c|c|}
\hline change in & exchange rate & $\begin{array}{r}\text { Eqn 1 } \\
0.000 \\
(0.000)\end{array}$ & $\begin{array}{r}\text { Eqn2 } \\
0.000 \\
(0.000)\end{array}$ & $\begin{array}{r}\text { Eqn3 } \\
0.000 \\
(0.000)\end{array}$ & $\begin{array}{c}\text { Eqn } 4 \\
0.000 \text { *** } \\
(0.000)\end{array}$ & $\begin{array}{c}\text { Eqn 5 } \\
0.000^{* * *} \\
(0.000)\end{array}$ & $\begin{array}{r}\text { Eqn 6 } \\
0.000 \\
(0.000)\end{array}$ & $\begin{array}{c}\text { Eqn } 7 \\
0.000 * \\
(0.000)\end{array}$ & $\begin{array}{c}\text { Eqn 8 } \\
0.000 * \\
(0.000)\end{array}$ & $\begin{array}{r}\text { Eqn 9 } \\
0.000 \\
(0.000)\end{array}$ \\
\hline change in & exporter's price & $\begin{array}{r}-0.346 \\
(0.762)\end{array}$ & $\begin{array}{l}0.935 * * \\
(0.386)\end{array}$ & $\begin{array}{l}0.9088^{* * *} \\
(0.349)\end{array}$ & $\begin{array}{r}0.160 \\
(0.152)\end{array}$ & $\begin{array}{r}0.143 \\
(0.128)\end{array}$ & $\begin{array}{r}0.097 \\
(0.153)\end{array}$ & $\begin{array}{r}0.145 \\
(0.144)\end{array}$ & $\begin{array}{r}0.152 \\
(0.142)\end{array}$ & $\begin{array}{r}0.100 \\
(0.150)\end{array}$ \\
\hline$($ delta s)* & trend & & $\begin{array}{l}0.122 * * * \\
(0.017)\end{array}$ & $\begin{array}{l}0.122 * * * \\
(0.023)\end{array}$ & $\begin{array}{r}-0.008 \\
(0.014)\end{array}$ & $\begin{array}{l}-0.011^{* *} \\
(0.005)\end{array}$ & $\begin{array}{l}-0.048^{* * *} \\
(0.013)\end{array}$ & $\begin{array}{r}-0.035 \\
(0.023)\end{array}$ & $\begin{array}{r}-0.035 \\
(0.023)\end{array}$ & $\begin{array}{l}-0.048^{* * *} \\
(0.014)\end{array}$ \\
\hline$($ delta s)* & $\begin{array}{l}\log [\text { per capitaRGDP(importer) / } \\
\text { per capitaRGDP(exporter)] }\end{array}$ & & & $\begin{array}{r}-0.003 \\
(0.043)\end{array}$ & $\begin{array}{r}-0.003 \\
(0.012)\end{array}$ & $\begin{array}{l}-0.079^{* * *} \\
(0.016)\end{array}$ & $\begin{array}{l}-0.188 * * * \\
(0.030)\end{array}$ & $\begin{array}{l}-0.089 * * \\
(0.042)\end{array}$ & $\begin{array}{l}-0.107 * * * \\
(0.041)\end{array}$ & $\begin{array}{l}-0.1911^{* * *} \\
(0.032)\end{array}$ \\
\hline$($ delta s)* & tariff levels & & & & $\begin{array}{l}-0.223 \text { ** } \\
(0.114)\end{array}$ & $\begin{array}{l}-0.373^{* * *} \\
(0.048)\end{array}$ & $\begin{array}{l}-0.502 \text { *** } \\
(0.049)\end{array}$ & $\begin{array}{l}-0.569 * * * \\
(0.057)\end{array}$ & $\begin{array}{l}-0.573 \text { *** } \\
(0.055)\end{array}$ & $\begin{array}{l}-0.510^{* * *} \\
(0.051)\end{array}$ \\
\hline$($ delta s)* & log distance & & & & $\begin{array}{l}0.167^{* * *} \\
(0.033)\end{array}$ & $\begin{array}{l}0.210^{* * *} \\
(0.013)^{*}\end{array}$ & $\begin{array}{l}0.275^{* * *} \\
(0.020)\end{array}$ & $\begin{array}{l}0.259^{* * *} \\
(0.023)^{*}\end{array}$ & $\begin{array}{l}0.2622^{* * *} \\
(0.023)^{2}\end{array}$ & $\begin{array}{l}0.277^{* * *} \\
(0.020)\end{array}$ \\
\hline$($ delta s)* & $\log [$ RGDP(importer)/RGDP(exporter)] & & & & & $\begin{array}{l}0.1011^{* * *} \\
(0.017)\end{array}$ & $\begin{array}{l}0.157^{* * *} \\
(0.030)\end{array}$ & $\begin{array}{r}0.024 \\
(0.044)\end{array}$ & $\begin{array}{r}0.051 \\
(0.043)\end{array}$ & $\begin{array}{l}0.160 \text { *** } \\
(0.035)\end{array}$ \\
\hline$($ delta s)* & log real wage $(\$)$ & & & & & & $\begin{array}{l}-0.0511^{* * *} \\
(0.013)\end{array}$ & $\begin{array}{l}-0.067^{* * *} \\
(0.012)\end{array}$ & $\begin{array}{l}-0.0622^{* * *} \\
(0.012)\end{array}$ & $\begin{array}{l}-0.0511^{* * *} \\
(0.013)\end{array}$ \\
\hline$($ delta s)* & long term inflation & & & & & & & $\begin{array}{l}0.144^{* * *} \\
(0.045)\end{array}$ & $\begin{array}{l}0.119^{* * *} \\
(0.043)\end{array}$ & $\begin{array}{r}-0.005 \\
(0.036)\end{array}$ \\
\hline$($ delta s)* & long term exchange rate variability & & & & & & & $\begin{array}{c}0.000 * \\
(0.000)\end{array}$ & $\begin{array}{c}0.000 * \\
(0.000)\end{array}$ & \\
\hline$($ delta s)* & US Importer dummy & & & & & & & & $\begin{array}{l}-1.259^{* * *} \\
(0.246)\end{array}$ & $\begin{array}{l}-1.644^{* * *} \\
(0.242)\end{array}$ \\
\hline & Error Correction term (ECM) & $\begin{array}{r}-0.265 \\
(0.224)\end{array}$ & $\begin{array}{l}-0.492 * * * \\
(0.126)\end{array}$ & $\begin{array}{r}-0.266 \\
(0.193)\end{array}$ & $\begin{array}{r}-0.041 \\
(0.391)\end{array}$ & $\begin{array}{r}0.115 \\
(0.339)\end{array}$ & $\begin{array}{r}-0.102 \\
(0.405)\end{array}$ & $\begin{array}{r}-0.602 \\
(0.416)\end{array}$ & $\begin{array}{r}-0.570 \\
(0.408)\end{array}$ & $\begin{array}{r}-0.076 \\
(0.403)\end{array}$ \\
\hline $\mathrm{ECM}^{*}$ & trend & & $\begin{array}{r}-0.013 \\
(0.010)\end{array}$ & $\begin{array}{r}-0.013 \\
(0.010)\end{array}$ & $\begin{array}{r}0.001 \\
(0.005)\end{array}$ & $\begin{array}{r}0.002 \\
(0.005)\end{array}$ & $\begin{array}{r}-0.001 \\
(0.005)\end{array}$ & $\begin{array}{r}0.003 \\
(0.005)\end{array}$ & $\begin{array}{r}0.002 \\
(0.004)\end{array}$ & $\begin{array}{r}0.001 \\
(0.005)\end{array}$ \\
\hline ECM* & $\begin{array}{l}\log [\text { per capitaRGDP(importer) / } \\
\text { per capitaRGDP(exporter)] }\end{array}$ & & & $\begin{array}{l}0.068 * \\
(0.038)\end{array}$ & $\begin{array}{l}0.023 * \\
(0.013)\end{array}$ & $\begin{array}{r}0.025 \\
(0.035)\end{array}$ & $\begin{array}{r}0.044 \\
(0.050)\end{array}$ & $\begin{array}{r}0.071 \\
(0.055)\end{array}$ & $\begin{array}{r}0.064 \\
(0.058)\end{array}$ & $\begin{array}{r}0.075 \\
(0.054)\end{array}$ \\
\hline $\mathrm{ECM}^{*}$ & tariff levels & & & & $\begin{array}{l}0.159 * * * \\
(0.051)\end{array}$ & $\begin{array}{l}0.155^{* * *} \\
(0.046)\end{array}$ & $\begin{array}{l}0.102 * \\
(0.059)\end{array}$ & $\begin{array}{l}0.150 * * \\
(0.060)\end{array}$ & $\begin{array}{l}0.146^{* *} \\
(0.061)\end{array}$ & $\begin{array}{l}0.102 * \\
(0.060)\end{array}$ \\
\hline $\mathrm{ECM}^{*}$ & log distance & & & & $\begin{array}{l}-0.091 * * \\
(0.045)\end{array}$ & $\begin{array}{l}-0.110^{* * *} \\
(0.040)\end{array}$ & $\begin{array}{r}-0.075 \\
(0.047)\end{array}$ & $\begin{array}{r}-0.059 \\
(0.045)\end{array}$ & $\begin{array}{r}-0.058 \\
(0.045)\end{array}$ & $\begin{array}{l}-0.084 * \\
(0.047)\end{array}$ \\
\hline $\mathrm{ECM}^{*}$ & $\log [$ RGDP(importer)/RGDP(exporter) $]$ & & & & & $\begin{array}{r}-0.005 \\
(0.035)\end{array}$ & $\begin{array}{r}0.009 \\
(0.055)\end{array}$ & $\begin{array}{r}0.018 \\
(0.050)\end{array}$ & $\begin{array}{r}0.024 \\
(0.057)\end{array}$ & $\begin{array}{r}-0.013 \\
(0.057)\end{array}$ \\
\hline $\mathrm{ECM}^{*}$ & log real wage $(\$)$ & & & & & & $\begin{array}{r}0.034 \\
(0.031)\end{array}$ & $\begin{array}{l}0.092^{* * *} \\
(0.031)\end{array}$ & $\begin{array}{l}0.089^{* * *} \\
(0.031)\end{array}$ & $\begin{array}{r}0.036 \\
(0.030)\end{array}$ \\
\hline $\mathrm{ECM}^{*}$ & long term inflation & & & & & & & $\begin{array}{r}0.130 \\
(0.112)\end{array}$ & $\begin{array}{r}0.116 \\
(0.110)\end{array}$ & $\begin{array}{r}0.120 \\
(0.109)\end{array}$ \\
\hline $\mathrm{ECM}^{*}$ & long term exchange rate variability & & & & & & & $\begin{array}{r}0.058 \\
(0.527)\end{array}$ & $\begin{array}{r}-0.075 \\
(0.522)\end{array}$ & \\
\hline $\mathrm{ECM}^{*}$ & US Importer dummy & & & & & & & & $\begin{array}{r}0.065 \\
(0.225)\end{array}$ & $\begin{array}{r}0.153 \\
(0.242)\end{array}$ \\
\hline & \# of Observations & 647 & 647 & 647 & 540 & 540 & 368 & 360 & 360 & 368 \\
\hline & Adjusted R-squared & -0.027 & 0.748 & 0.751 & 0.961 & 0.971 & 0.974 & 0.972 & 0.972 & 0.975 \\
\hline & Country dummies & yes & yes & yes & yes & yes & yes & yes & yes & yes \\
\hline & Product dummies & yes & yes & yes & yes & yes & yes & yes & yes & yes \\
\hline
\end{tabular}

Full sample

Product dummies

$*=10 \%, * *=5 \%$, and $* * *=1 \%$ levels of significance

product specific (ps) tariffs 
Table a8: Pass-through to Import price of Vermouth

\begin{tabular}{|c|c|c|c|c|c|c|c|c|c|c|}
\hline change in & exchange rate & $\begin{array}{r}\text { Eqn 1 } \\
0.000 \\
(0.000)\end{array}$ & $\begin{array}{r}\text { Eqn2 } \\
0.000 \\
(0.000)\end{array}$ & $\begin{array}{c}\text { Eqn3 } \\
0.000 * \\
(0.000)\end{array}$ & $\begin{array}{r}\text { Eqn } 4 \\
0.000 \\
(0.000)\end{array}$ & $\begin{array}{r}\text { Eqn } 5 \\
0.000 \\
(0.000)\end{array}$ & $\begin{array}{r}\text { Eqn 6 } \\
0.000 \\
(0.000)\end{array}$ & $\begin{array}{c}\text { Eqn } 7 \\
0.000^{* * *} \\
(0.000)\end{array}$ & $\begin{array}{c}\text { Eqn 8 } \\
0.000 * * * \\
(0.000)\end{array}$ & $\begin{array}{r}\text { Eqn 9 } \\
0.000 \\
(0.000)\end{array}$ \\
\hline change in & exporter's price & $\begin{array}{r}0.198 \\
(0.276)\end{array}$ & $\begin{array}{r}0.129 \\
(0.109)\end{array}$ & $\begin{array}{l}0.138 * \\
(0.077)\end{array}$ & $\begin{array}{r}-0.087 \\
(0.065)\end{array}$ & $\begin{array}{r}-0.100 \\
(0.063)\end{array}$ & $\begin{array}{r}0.026 \\
(0.070)\end{array}$ & $\begin{array}{r}0.012 \\
(0.066)\end{array}$ & $\begin{array}{r}0.021 \\
(0.066)\end{array}$ & $\begin{array}{r}0.030 \\
(0.070)\end{array}$ \\
\hline$($ delta s)* & trend & & $\begin{array}{l}0.1433^{* * *} \\
(0.023)^{*}\end{array}$ & $\begin{array}{l}0.104^{* * *} \\
(0.008)\end{array}$ & $\begin{array}{r}0.014 \\
(0.020)\end{array}$ & $\begin{array}{r}-0.009 \\
(0.018)\end{array}$ & $\begin{array}{l}-0.077^{* * *} \\
(0.018)\end{array}$ & $\begin{array}{l}-0.1988^{* * *} \\
(0.038)\end{array}$ & $\begin{array}{l}-0.197 * * * \\
(0.038)\end{array}$ & $\begin{array}{l}-0.088^{* * *} \\
(0.020)\end{array}$ \\
\hline$($ delta s)* & $\begin{array}{l}\log [\text { per capitaRGDP(importer) / } \\
\text { per capitaRGDP(exporter)] }\end{array}$ & & & $\begin{array}{l}0.089 * * * \\
(0.013)\end{array}$ & $\begin{array}{l}-0.021 \\
(0.017)\end{array}$ & $\begin{array}{l}-0.076^{* *} \\
(0.032)\end{array}$ & $\begin{array}{l}-0.126^{* *} \\
(0.052)\end{array}$ & $\begin{array}{r}-0.025 \\
(0.066)\end{array}$ & $\begin{array}{l}-0.042 \\
(0.068)\end{array}$ & $\begin{array}{l}-0.155^{* * *} \\
(0.050)\end{array}$ \\
\hline$($ delta s)* & tariff levels & & & & $\begin{array}{l}-0.207 \\
(0.135)\end{array}$ & $\begin{array}{l}-0.361 * * * \\
(0.139)\end{array}$ & $\begin{array}{l}-0.804^{* * *} \\
(0.088)\end{array}$ & $\begin{array}{l}-0.8088^{* * *} \\
(0.096)\end{array}$ & $\begin{array}{l}-0.807 * * * \\
(0.097)\end{array}$ & $\begin{array}{l}-0.789 \text { *** } \\
(0.086)\end{array}$ \\
\hline$($ delta s)* & $\log$ distance & & & & $\begin{array}{l}0.163 \text { *** } \\
(0.043)\end{array}$ & $\begin{array}{l}0.209 \text { *** } \\
(0.042)\end{array}$ & $\begin{array}{l}0.438^{* * *} \\
(0.041)\end{array}$ & $\begin{array}{l}0.526 \text { *** } \\
(0.051)\end{array}$ & $\begin{array}{l}0.5222^{* * *} \\
(0.052)\end{array}$ & $\begin{array}{l}0.449 \text { *** } \\
(0.047)\end{array}$ \\
\hline$($ delta s)* & $\log [$ RGDP(importer)/RGDP(exporter)] & & & & & $\begin{array}{l}0.0611^{* *} \\
(0.027)\end{array}$ & $\begin{array}{r}0.000 \\
(0.057)\end{array}$ & $\begin{array}{r}-0.090 \\
(0.075)\end{array}$ & $\begin{array}{r}-0.068 \\
(0.078)\end{array}$ & $\begin{array}{r}0.029 \\
(0.049)\end{array}$ \\
\hline$($ delta s)* & log real wage $(\$)$ & & & & & & $\begin{array}{l}-0.0611^{* * *} \\
(0.016)\end{array}$ & $\begin{array}{l}-0.0622^{* * *} \\
(0.019)\end{array}$ & $\begin{array}{l}-0.058 * * * \\
(0.020)\end{array}$ & $\begin{array}{l}-0.065^{* * *} \\
(0.019)\end{array}$ \\
\hline$($ delta s)* & long term inflation & & & & & & & $\begin{array}{l}-0.119^{* *} \\
(0.058)\end{array}$ & $\begin{array}{l}-0.130 * * \\
(0.058)\end{array}$ & $\begin{array}{r}-0.050 \\
(0.040)\end{array}$ \\
\hline$($ delta s)* & long term exchange rate variability & & & & & & & $\begin{array}{l}0.000 \text { *** } \\
(0.000)\end{array}$ & $\begin{array}{l}0.000 \text { *** } \\
(0.000)\end{array}$ & \\
\hline$($ delta s)* & US Importer dummy & & & & & & & & $\begin{array}{l}-0.999 * * \\
(0.421)\end{array}$ & $\begin{array}{l}-1.240 * * * \\
(0.322)\end{array}$ \\
\hline & Error Correction term (ECM) & $\begin{array}{l}-0.228 * \\
(0.128)\end{array}$ & $\begin{array}{l}-0.254 * \\
(0.133)\end{array}$ & $\begin{array}{l}-0.4844^{* * *} \\
(0.106)\end{array}$ & $\begin{array}{l}-0.556 * \\
(0.314)\end{array}$ & $\begin{array}{l}-0.608^{* *} \\
(0.289)\end{array}$ & $\begin{array}{l}-0.8477^{* *} \\
(0.387)\end{array}$ & $\begin{array}{l}-1.047^{* * *} \\
(0.373)\end{array}$ & $\begin{array}{l}-1.139 \text { *** } \\
(0.374)\end{array}$ & $\begin{array}{l}-0.693 * \\
(0.401)\end{array}$ \\
\hline ECM* & trend & & $\begin{array}{r}-0.006 \\
(0.012)\end{array}$ & $\begin{array}{r}0.006 \\
(0.005)\end{array}$ & $\begin{array}{r}0.002 \\
(0.005)\end{array}$ & $\begin{array}{r}0.003 \\
(0.005)\end{array}$ & $\begin{array}{r}0.000 \\
(0.007)\end{array}$ & $\begin{array}{r}-0.005 \\
(0.005)\end{array}$ & $\begin{array}{r}-0.006 \\
(0.005)\end{array}$ & $\begin{array}{r}0.000 \\
(0.006)\end{array}$ \\
\hline ECM* & $\begin{array}{l}\log [\text { per capitaRGDP(importer) / } \\
\text { per capitaRGDP(exporter)] }\end{array}$ & & & $\begin{array}{l}0.040 * * \\
(0.016)\end{array}$ & $\begin{array}{r}0.010 \\
(0.011)\end{array}$ & $\begin{array}{l}0.047 * \\
(0.026)\end{array}$ & $\begin{array}{r}0.054 \\
(0.042)\end{array}$ & $\begin{array}{r}0.008 \\
(0.034)\end{array}$ & $\begin{array}{r}0.001 \\
(0.035)\end{array}$ & $\begin{array}{r}0.056 \\
(0.042)\end{array}$ \\
\hline $\mathrm{ECM}^{*}$ & tariff levels & & & & $\begin{array}{r}-0.002 \\
(0.035)\end{array}$ & $\begin{array}{r}0.013 \\
(0.033)\end{array}$ & $\begin{array}{l}0.078 * \\
(0.042)\end{array}$ & $\begin{array}{l}0.089^{* *} \\
(0.039)\end{array}$ & $\begin{array}{l}0.086^{* *} \\
(0.039)\end{array}$ & $\begin{array}{l}0.071 * \\
(0.041)\end{array}$ \\
\hline $\mathrm{ECM}^{*}$ & $\log$ distance & & & & $\begin{array}{r}0.031 \\
(0.034)\end{array}$ & $\begin{array}{r}0.030 \\
(0.032)\end{array}$ & $\begin{array}{r}0.016 \\
(0.037)\end{array}$ & $\begin{array}{r}0.035 \\
(0.036)\end{array}$ & $\begin{array}{r}0.042 \\
(0.036)\end{array}$ & $\begin{array}{r}0.006 \\
(0.037)\end{array}$ \\
\hline $\mathrm{ECM}^{*}$ & $\log [$ RGDP(importer)/RGDP(exporter)] & & & & & $\begin{array}{r}-0.035 \\
(0.029)\end{array}$ & $\begin{array}{r}-0.018 \\
(0.038)\end{array}$ & $\begin{array}{r}0.038 \\
(0.033)\end{array}$ & $\begin{array}{r}0.053 \\
(0.036)\end{array}$ & $\begin{array}{r}-0.024 \\
(0.041)\end{array}$ \\
\hline $\mathrm{ECM}^{*}$ & log real wage $(\$)$ & & & & & & $\begin{array}{r}0.032 \\
(0.024)\end{array}$ & $\begin{array}{l}0.050 * * \\
(0.021)\end{array}$ & $\begin{array}{l}0.059 * * * \\
(0.020)\end{array}$ & $\begin{array}{r}0.023 \\
(0.022)\end{array}$ \\
\hline $\mathrm{ECM}^{*}$ & long term inflation & & & & & & & $\begin{array}{r}-0.093 \\
(0.096)\end{array}$ & $\begin{array}{r}-0.098 \\
(0.094)\end{array}$ & $\begin{array}{r}-0.006 \\
(0.104)\end{array}$ \\
\hline $\mathrm{ECM}^{*}$ & long term exchange rate variability & & & & & & & $\begin{array}{c}0.763 * \\
(0.412)\end{array}$ & $\begin{array}{c}0.704 * \\
(0.422)\end{array}$ & \\
\hline $\mathrm{ECM}^{*}$ & US Importer dummy & & & & & & & & $\begin{array}{r}-0.257 \\
(0.187)\end{array}$ & $\begin{array}{r}0.043 \\
(0.202)\end{array}$ \\
\hline & \# of Observations & 679 & 679 & 679 & 547 & 547 & 372 & 363 & 363 & 372 \\
\hline & Adjusted R-squared & -0.023 & 0.743 & 0.866 & 0.935 & 0.938 & 0.950 & 0.948 & 0.948 & 0.950 \\
\hline & Country dummies & yes & yes & yes & yes & yes & yes & yes & yes & yes \\
\hline & Product dummies & yes & yes & yes & yes & yes & yes & yes & yes & yes \\
\hline
\end{tabular}

Full sample

Product dummies

$-0.228 *$
$(0.128)$

-0.254
$(0.133)$

-0.484 ***

-0.556 *

-0.608 **

-0.847 *

$-1.047$

$-1.139$

$-0.693 *$

$-0.006$

0.006
$(0.005)$

0.002

0.003

0.000

$-0.005$

$-0.006$

0.000

$0.040 * *$

(0.011)

$0.047 *$

0.054

0.008

0.001

0.056

-0.002
$(0.035)$

0.01

0.078

0.089

$0.086 *$

0.071

0.031
$(0.034)$

yes

product specific (ps) tariffs 
Table b1: Pass-through to Local competitor's price of Marlboro

\begin{tabular}{|c|c|c|c|c|c|c|c|c|c|c|}
\hline change in & exchange rate & $\begin{array}{c}\text { Eqn 1 } \\
0.973 * * * \\
(0.010)\end{array}$ & $\begin{array}{c}\text { Eqn2 } \\
0.999 \\
(0.022)\end{array}$ & $\begin{array}{c}\text { Eqn3 } \\
0.900 * * * \\
(0.049)\end{array}$ & $\begin{array}{c}\text { Eqn 4 } \\
1.095^{* * *} \\
(0.286)\end{array}$ & $\begin{array}{c}\text { Eqn 5 } \\
1.1222^{* * *} \\
(0.299)\end{array}$ & $\begin{array}{c}\text { Eqn 6 } \\
2.539 * \\
(1.515)\end{array}$ & $\begin{array}{c}\text { Eqn } 7 \\
2.928 * \\
(1.559)\end{array}$ & $\begin{array}{c}\text { Eqn 8 } \\
2.928 * \\
(1.559)\end{array}$ & $\begin{array}{c}\text { Eqn 9 } \\
2.592 * \\
(1.506)\end{array}$ \\
\hline change in & exporter's price & $\begin{array}{r}0.023 \\
(0.049)\end{array}$ & $\begin{array}{r}0.047 \\
(0.049)\end{array}$ & $\begin{array}{r}0.049 \\
(0.048)\end{array}$ & $\begin{array}{r}0.052 \\
(0.050)\end{array}$ & $\begin{array}{r}0.051 \\
(0.049)\end{array}$ & $\begin{array}{l}0.112 * * \\
(0.051)\end{array}$ & $\begin{array}{c}0.095 * \\
(0.050)\end{array}$ & $\begin{array}{c}0.095 * \\
(0.050)\end{array}$ & $\begin{array}{l}0.129 * * \\
(0.053)\end{array}$ \\
\hline$($ delta s)* & trend & & $\begin{array}{r}-0.004 \\
(0.003)\end{array}$ & $\begin{array}{l}-0.016^{* *} \\
(0.008)\end{array}$ & $\begin{array}{l}-0.030 * * * \\
(0.010)\end{array}$ & $\begin{array}{l}-0.033^{* * *} \\
(0.010)\end{array}$ & $\begin{array}{l}-0.116^{* * *} \\
(0.015)\end{array}$ & $\begin{array}{l}-0.118 * * * \\
(0.020)\end{array}$ & $\begin{array}{l}-0.118^{* * *} \\
(0.020)\end{array}$ & $\begin{array}{l}-0.127^{* * *} \\
(0.018)\end{array}$ \\
\hline$($ delta s)* & $\begin{array}{l}\log [\text { per capitaRGDP(importer) / } \\
\text { per capitaRGDP(exporter)] }\end{array}$ & & & $\begin{array}{l}-0.115^{* *} \\
(0.057)\end{array}$ & $\begin{array}{l}-0.205 * * \\
(0.091)\end{array}$ & $\begin{array}{l}-0.215^{* *} \\
(0.089)\end{array}$ & $\begin{array}{l}-0.4911^{* * *} \\
(0.098)\end{array}$ & $\begin{array}{l}-0.439 * * * \\
(0.099)\end{array}$ & $\begin{array}{l}-0.439 * * * \\
(0.099)\end{array}$ & $\begin{array}{l}-0.506 * * * \\
(0.098)\end{array}$ \\
\hline$($ delta s)* & tariff levels & & & & $\begin{array}{r}-0.042 \\
(0.032)\end{array}$ & $\begin{array}{r}-0.040 \\
(0.034)\end{array}$ & $\begin{array}{l}-0.130 * * \\
(0.054)\end{array}$ & $\begin{array}{r}-0.008 \\
(0.089)\end{array}$ & $\begin{array}{r}-0.008 \\
(0.089)\end{array}$ & $\begin{array}{r}-0.077 \\
(0.078)\end{array}$ \\
\hline$($ delta s)* & $\log$ distance & & & & $\begin{array}{r}-0.019 \\
(0.027)\end{array}$ & $\begin{array}{r}-0.026 \\
(0.031)\end{array}$ & $\begin{array}{r}-0.135 \\
(0.164)\end{array}$ & $\begin{array}{r}-0.159 \\
(0.171)\end{array}$ & $\begin{array}{r}-0.159 \\
(0.171)\end{array}$ & $\begin{array}{r}-0.115 \\
(0.166)\end{array}$ \\
\hline$($ delta s)* & $\log [$ RGDP(importer)/RGDP(exporter)] & & & & & $\begin{array}{r}-0.009 \\
(0.011)\end{array}$ & $\begin{array}{r}0.040 \\
(0.052)\end{array}$ & $\begin{array}{r}0.098 \\
(0.071)\end{array}$ & $\begin{array}{r}0.098 \\
(0.071)\end{array}$ & $\begin{array}{r}0.106 \\
(0.073)\end{array}$ \\
\hline$($ delta s)* & log real wage $(\$)$ & & & & & & $\begin{array}{l}-0.035^{* * *} \\
(0.006)\end{array}$ & $\begin{array}{l}-0.053 * * * \\
(0.006)\end{array}$ & $\begin{array}{l}-0.053 * * * \\
(0.006)\end{array}$ & $\begin{array}{l}-0.037 * * * \\
(0.006)\end{array}$ \\
\hline$($ delta s)* & long term inflation & & & & & & & $\begin{array}{r}-0.079 \\
(0.073)\end{array}$ & $\begin{array}{r}-0.079 \\
(0.073)\end{array}$ & $\begin{array}{r}-0.075 \\
(0.072)\end{array}$ \\
\hline$($ delta s)* & long term exchange rate variability & & & & & & & $\begin{array}{l}-2.165 * * * \\
(0.584)\end{array}$ & $\begin{array}{l}-2.165 * * * \\
(0.584)\end{array}$ & \\
\hline$($ delta s)* & US Importer dummy & & & & & & & & & \\
\hline & Error Correction term (ECM) & $\begin{array}{l}-0.143 \text { *** } \\
(0.026)\end{array}$ & $\begin{array}{l}-0.278^{* * * *} \\
(0.058)\end{array}$ & $\begin{array}{l}-0.247^{* * * *} \\
(0.065)\end{array}$ & $\begin{array}{r}-0.928 \\
(0.575)\end{array}$ & $\begin{array}{r}-0.912 \\
(0.571)\end{array}$ & $\begin{array}{r}-0.344 \\
(0.628)\end{array}$ & $\begin{array}{r}-0.333 \\
(0.633)\end{array}$ & $\begin{array}{r}-0.333 \\
(0.633)\end{array}$ & $\begin{array}{r}-0.351 \\
(0.628)\end{array}$ \\
\hline ECM* & trend & & $\begin{array}{l}0.012 \text { *** } \\
(0.004)\end{array}$ & $\begin{array}{l}0.012 \text { *** } \\
(0.004)\end{array}$ & $\begin{array}{l}0.010 \text { ** } \\
(0.005)\end{array}$ & $\begin{array}{c}0.009 * \\
(0.005)\end{array}$ & $\begin{array}{c}0.009 \\
(0.005)\end{array}$ & $\begin{array}{r}0.003 \\
(0.005)\end{array}$ & $\begin{array}{r}0.003 \\
(0.005)\end{array}$ & $\begin{array}{r}0.008 \\
(0.005)\end{array}$ \\
\hline $\mathrm{ECM}^{*}$ & $\begin{array}{l}\log [\text { per capitaRGDP(importer) / } \\
\text { per capitaRGDP(exporter)] }\end{array}$ & & & $\begin{array}{r}0.023 \\
(0.024)\end{array}$ & $\begin{array}{r}0.041 \\
(0.030)\end{array}$ & $\begin{array}{r}0.033 \\
(0.032)\end{array}$ & $\begin{array}{r}-0.006 \\
(0.045)\end{array}$ & $\begin{array}{r}-0.036 \\
(0.040)\end{array}$ & $\begin{array}{r}-0.036 \\
(0.040)\end{array}$ & $\begin{array}{r}-0.017 \\
(0.044)\end{array}$ \\
\hline $\mathrm{ECM}^{*}$ & tariff levels & & & & $\begin{array}{r}-0.018 \\
(0.031)\end{array}$ & $\begin{array}{r}-0.025 \\
(0.031)\end{array}$ & $\begin{array}{r}-0.053 \\
(0.039)\end{array}$ & $\begin{array}{r}-0.046 \\
(0.040)\end{array}$ & $\begin{array}{r}-0.046 \\
(0.040)\end{array}$ & $\begin{array}{r}-0.059 \\
(0.039)\end{array}$ \\
\hline ECM* & $\log$ distance & & & & $\begin{array}{r}0.088 \\
(0.066)\end{array}$ & $\begin{array}{r}0.095 \\
(0.065)\end{array}$ & $\begin{array}{r}0.041 \\
(0.074)\end{array}$ & $\begin{array}{r}0.048 \\
(0.073)\end{array}$ & $\begin{array}{r}0.048 \\
(0.073)\end{array}$ & $\begin{array}{r}0.040 \\
(0.074)\end{array}$ \\
\hline $\mathrm{ECM}^{*}$ & $\log [$ RGDP(importer)/RGDP(exporter)] & & & & & $\begin{array}{r}0.017 \\
(0.020)\end{array}$ & $\begin{array}{r}0.014 \\
(0.021)\end{array}$ & $\begin{array}{c}0.027 * \\
(0.016)\end{array}$ & $\begin{array}{c}0.027 \text { * } \\
(0.016)\end{array}$ & $\begin{array}{r}0.013 \\
(0.020)\end{array}$ \\
\hline $\mathrm{ECM}^{*}$ & log real wage $(\$)$ & & & & & & $\begin{array}{r}-0.013 \\
(0.014)\end{array}$ & $\begin{array}{r}-0.009 \\
(0.013)\end{array}$ & $\begin{array}{r}-0.009 \\
(0.013)\end{array}$ & $\begin{array}{r}-0.007 \\
(0.017)\end{array}$ \\
\hline $\mathrm{ECM}^{*}$ & long term inflation & & & & & & & $\begin{array}{c}0.141 * \\
(0.076)\end{array}$ & $\begin{array}{c}0.1411^{*} \\
(0.076)\end{array}$ & $\begin{array}{r}-0.055 \\
(0.095)\end{array}$ \\
\hline ECM* & long term exchange rate variability & & & & & & & $\begin{array}{l}-4.712^{* * *} \\
(0.865)\end{array}$ & $\begin{array}{l}-4.712 \text { **** } \\
(0.865)\end{array}$ & \\
\hline \multicolumn{11}{|c|}{ ECM* US Importer dummy } \\
\hline & \# of Observations & 731 & 731 & 731 & 595 & 595 & 363 & 362 & 362 & 363 \\
\hline & Adjusted R-squared & 0.940 & 0.941 & 0.942 & 0.945 & 0.945 & 0.962 & 0.967 & 0.967 & 0.962 \\
\hline & Country dummies & yes & yes & yes & yes & yes & yes & yes & yes & yes \\
\hline & Product dummies & yes & yes & yes & yes & yes & yes & yes & yes & yes \\
\hline
\end{tabular}


Table b2: Pass-through to Local competitor's price of Coca-Cola

\begin{tabular}{|c|c|c|c|c|c|c|c|c|c|c|}
\hline change in & exchange rate & $\begin{array}{c}\text { Eqn 1 } \\
0.987 * * * \\
(0.031)\end{array}$ & $\begin{array}{c}\text { Eqn2 } \\
0.977^{* * *} \\
(0.055)\end{array}$ & $\begin{array}{c}\text { Eqn3 } \\
0.972 \\
(0.095)\end{array}$ & $\begin{array}{c}\text { Eqn } 4 \\
2.146^{* * *} \\
(0.712)\end{array}$ & $\begin{array}{c}\text { Eqn 5 } \\
2.2033^{* * *} \\
(0.732)\end{array}$ & $\begin{array}{r}\text { Eqn 6 } \\
-2.628 \\
(4.570)\end{array}$ & $\begin{array}{r}\text { Eqn } 7 \\
3.491 \\
(3.622)\end{array}$ & $\begin{array}{r}\text { Eqn 8 } \\
3.491 \\
(3.622)\end{array}$ & $\begin{array}{r}\text { Eqn 9 } \\
-1.106 \\
(4.535)\end{array}$ \\
\hline change in & exporter's price & $\begin{array}{r}0.090 \\
(0.086)\end{array}$ & $\begin{array}{r}0.090 \\
(0.086)\end{array}$ & $\begin{array}{r}0.096 \\
(0.090)\end{array}$ & $\begin{array}{r}0.036 \\
(0.097)\end{array}$ & $\begin{array}{r}0.035 \\
(0.096)\end{array}$ & $\begin{array}{r}0.037 \\
(0.139)\end{array}$ & $\begin{array}{r}-0.068 \\
(0.123)\end{array}$ & $\begin{array}{r}-0.068 \\
(0.123)\end{array}$ & $\begin{array}{r}-0.019 \\
(0.129)\end{array}$ \\
\hline$($ delta s)* & trend & & $\begin{array}{r}0.002 \\
(0.006)\end{array}$ & $\begin{array}{r}0.001 \\
(0.020)\end{array}$ & $\begin{array}{r}-0.005 \\
(0.019)\end{array}$ & $\begin{array}{r}-0.002 \\
(0.020)\end{array}$ & $\begin{array}{r}0.007 \\
(0.044)\end{array}$ & $\begin{array}{l}-0.097^{* *} \\
(0.047)\end{array}$ & $\begin{array}{l}-0.097 * * \\
(0.047)\end{array}$ & $\begin{array}{r}-0.006 \\
(0.037)\end{array}$ \\
\hline$($ delta s)* & $\begin{array}{l}\log [\text { per capitaRGDP(importer) / } \\
\text { per capitaRGDP(exporter)] }\end{array}$ & & & $\begin{array}{r}-0.007 \\
(0.150)\end{array}$ & $\begin{array}{r}0.005 \\
(0.168)\end{array}$ & $\begin{array}{r}0.023 \\
(0.168)\end{array}$ & $\begin{array}{r}0.038 \\
(0.295)\end{array}$ & $\begin{array}{l}-0.565^{* * *} \\
(0.157)^{*}\end{array}$ & $\begin{array}{l}-0.565 * * * \\
(0.157)\end{array}$ & $\begin{array}{r}-0.175 \\
(0.249)\end{array}$ \\
\hline$($ delta s)* & tariff levels & & & & $\begin{array}{r}-0.065 \\
(0.066)\end{array}$ & $\begin{array}{r}-0.066 \\
(0.066)\end{array}$ & $\begin{array}{r}-0.118 \\
(0.115)\end{array}$ & $\begin{array}{l}-0.499^{* *} \\
(0.203)\end{array}$ & $\begin{array}{l}-0.499 * * \\
(0.203)\end{array}$ & $\begin{array}{r}-0.043 \\
(0.171)\end{array}$ \\
\hline$($ delta s)* & log distance & & & & $\begin{array}{l}-0.113 * \\
(0.065)\end{array}$ & $\begin{array}{r}-0.117 \\
(0.075)\end{array}$ & $\begin{array}{r}0.402 \\
(0.511)\end{array}$ & $\begin{array}{r}-0.239 \\
(0.364)\end{array}$ & $\begin{array}{r}-0.239 \\
(0.364)\end{array}$ & $\begin{array}{r}0.200 \\
(0.504)\end{array}$ \\
\hline$($ delta s)* & log real wage $(\$)$ & & & & & & $\begin{array}{r}0.027 \\
(0.029)\end{array}$ & $\begin{array}{l}0.057^{* * *} \\
(0.016)\end{array}$ & $\begin{array}{l}0.057^{* * *} \\
(0.016)\end{array}$ & $\begin{array}{r}0.029 \\
(0.025)\end{array}$ \\
\hline$($ delta s)* & long term inflation & & & & & & & $\begin{array}{r}-0.223 \\
(0.165)\end{array}$ & $\begin{array}{r}-0.223 \\
(0.165)\end{array}$ & $\begin{array}{r}-0.025 \\
(0.113)\end{array}$ \\
\hline$($ delta s)* & long term exchange rate variability & & & & & & & $\begin{array}{l}3.556 \text { *** } \\
(1.049)\end{array}$ & $\begin{array}{l}3.556 \text { *** } \\
(1.049)\end{array}$ & \\
\hline$($ delta s)* & US Importer dummy & & & & & & & & & \\
\hline & Error Correction term (ECM) & $\begin{array}{l}-0.179^{* * *} \\
(0.055)\end{array}$ & $\begin{array}{r}-0.137 \\
(0.096)\end{array}$ & $\begin{array}{l}-0.251 * * \\
(0.117)\end{array}$ & $\begin{array}{r}-0.330 \\
(0.835)\end{array}$ & $\begin{array}{r}-0.104 \\
(0.844)\end{array}$ & $\begin{array}{r}0.128 \\
(0.959)\end{array}$ & $\begin{array}{r}0.139 \\
(0.946)\end{array}$ & $\begin{array}{r}0.139 \\
(0.946)\end{array}$ & $\begin{array}{r}0.652 \\
(0.992)\end{array}$ \\
\hline ECM* & trend & & $\begin{array}{r}-0.007 \\
(0.012)\end{array}$ & $\begin{array}{r}-0.007 \\
(0.012)\end{array}$ & $\begin{array}{r}-0.014 \\
(0.014)\end{array}$ & $\begin{array}{r}-0.016 \\
(0.014)\end{array}$ & $\begin{array}{c}-0.002 \\
(0.016)\end{array}$ & $\begin{array}{r}0.006 \\
(0.015)\end{array}$ & $\begin{array}{r}0.006 \\
(0.015)\end{array}$ & $\begin{array}{r}-0.009 \\
(0.016)\end{array}$ \\
\hline $\mathrm{ECM}^{*}$ & tariff levels & & & & $\begin{array}{r}0.090 \\
(0.070)\end{array}$ & $\begin{array}{r}0.078 \\
(0.067)\end{array}$ & $\begin{array}{r}0.036 \\
(0.102)\end{array}$ & $\begin{array}{r}-0.060 \\
(0.086)\end{array}$ & $\begin{array}{r}-0.060 \\
(0.086)\end{array}$ & $\begin{array}{r}0.036 \\
(0.096)\end{array}$ \\
\hline $\mathrm{ECM}^{*}$ & log distance & & & & $\begin{array}{r}-0.013 \\
(0.097)\end{array}$ & $\begin{array}{r}-0.015 \\
(0.097)\end{array}$ & $\begin{array}{r}-0.046 \\
(0.124)\end{array}$ & $\begin{array}{r}0.027 \\
(0.114)\end{array}$ & $\begin{array}{r}0.027 \\
(0.114)\end{array}$ & $\begin{array}{r}-0.078 \\
(0.124)\end{array}$ \\
\hline $\mathrm{ECM}^{*}$ & $\log [$ RGDP(importer)/RGDP(exporter)] & & & & & $\begin{array}{r}0.050 \\
(0.047)\end{array}$ & $\begin{array}{r}-0.007 \\
(0.076)\end{array}$ & $\begin{array}{r}0.046 \\
(0.081)\end{array}$ & $\begin{array}{r}0.046 \\
(0.081)\end{array}$ & $\begin{array}{r}0.030 \\
(0.072)\end{array}$ \\
\hline $\mathrm{ECM}^{*}$ & log real wage $(\$)$ & & & & & & $\begin{array}{r}-0.016 \\
(0.032)\end{array}$ & $\begin{array}{l}-0.046 * \\
(0.028)\end{array}$ & $\begin{array}{l}-0.046 * \\
(0.028)\end{array}$ & $\begin{array}{r}-0.011 \\
(0.032)\end{array}$ \\
\hline $\mathrm{ECM}^{*}$ & long term inflation & & & & & & & $\begin{array}{r}-0.072 \\
(0.227)\end{array}$ & $\begin{array}{r}-0.072 \\
(0.227)\end{array}$ & $\begin{array}{c}-0.503 * \\
(0.299)\end{array}$ \\
\hline $\mathrm{ECM}^{*}$ & long term exchange rate variability & & & & & & & $\begin{array}{l}-4.022 \\
(2.577)\end{array}$ & $\begin{array}{l}-4.022 \\
(2.577)\end{array}$ & \\
\hline
\end{tabular}

$\mathrm{ECM}^{*}$ US Importer dummy

\begin{tabular}{|c|c|c|c|c|c|c|c|c|c|}
\hline \# of Observations & 762 & 762 & 762 & 614 & 614 & 380 & 372 & 372 & 380 \\
\hline Adjusted R-squared & 0.851 & 0.850 & 0.850 & 0.860 & 0.860 & 0.845 & 0.842 & 0.842 & 0.849 \\
\hline Country dummies & yes & yes & yes & yes & yes & yes & yes & yes & yes \\
\hline Product dummies & yes & yes & yes & yes & yes & yes & yes & yes & yes \\
\hline
\end{tabular}


Table b3: Pass-through to Local competitor's price of Cognac

\begin{tabular}{|c|c|c|c|c|c|c|c|c|c|c|}
\hline change in & exchange rate & $\begin{array}{r}\text { Eqn 1 } \\
0.000 \\
(0.000)\end{array}$ & $\begin{array}{c}\text { Eqn2 } \\
0.000 * * \\
(0.000)\end{array}$ & $\begin{array}{r}\text { Eqn3 } \\
0.000 \\
(0.000)\end{array}$ & $\begin{array}{r}\text { Eqn } 4 \\
0.000 \\
(0.000)\end{array}$ & $\begin{array}{r}\text { Eqn 5 } \\
0.000 \\
(0.000)\end{array}$ & $\begin{array}{r}\text { Eqn 6 } \\
0.000 \\
(0.000)\end{array}$ & $\begin{array}{r}\text { Eqn } 7 \\
0.000 \\
(0.000)\end{array}$ & $\begin{array}{r}\text { Eqn 8 } \\
0.000 \\
(0.000)\end{array}$ & $\begin{array}{r}\text { Eqn 9 } \\
0.000 \\
(0.000)\end{array}$ \\
\hline change in & exporter's price & $\begin{array}{r}-0.044 \\
(0.167)\end{array}$ & $\begin{array}{l}0.278 * * * \\
(0.086)\end{array}$ & $\begin{array}{l}0.279^{* * *} \\
(0.082)\end{array}$ & $\begin{array}{l}0.216^{* * *} \\
(0.069)\end{array}$ & $\begin{array}{l}0.156 * * \\
(0.067)\end{array}$ & $\begin{array}{r}0.089 \\
(0.084)\end{array}$ & $\begin{array}{r}0.069 \\
(0.076)\end{array}$ & $\begin{array}{r}0.069 \\
(0.076)\end{array}$ & $\begin{array}{r}0.080 \\
(0.085)\end{array}$ \\
\hline$($ delta s)* & trend & & $\begin{array}{l}0.144^{* * * *} \\
(0.022)\end{array}$ & $\begin{array}{l}0.167^{* * *} \\
(0.022)^{*}\end{array}$ & $\begin{array}{l}0.030 * \\
(0.017)\end{array}$ & $\begin{array}{r}-0.001 \\
(0.018)\end{array}$ & $\begin{array}{l}-0.077^{* * *} \\
(0.018)\end{array}$ & $\begin{array}{l}-0.1211^{* * *} \\
(0.031)\end{array}$ & $\begin{array}{l}-0.121 * * * \\
(0.031)\end{array}$ & $\begin{array}{l}-0.072 * * * \\
(0.017)\end{array}$ \\
\hline$($ delta s)* & $\begin{array}{l}\log [\text { per capitaRGDP(importer) / } \\
\text { per capitaRGDP(exporter)] }\end{array}$ & & & $\begin{array}{r}0.060 \\
(0.052)\end{array}$ & $\begin{array}{r}0.013 \\
(0.013)\end{array}$ & $\begin{array}{l}-0.067^{* * *} \\
(0.023)\end{array}$ & $\begin{array}{l}-0.241 * * * \\
(0.040)\end{array}$ & $\begin{array}{r}-0.075 \\
(0.082)\end{array}$ & $\begin{array}{r}-0.070 \\
(0.084)\end{array}$ & $\begin{array}{l}-0.226 * * * \\
(0.042)\end{array}$ \\
\hline$($ delta s)* & tariff levels & & & & $\begin{array}{r}-0.048 \\
(0.084)\end{array}$ & $\begin{array}{l}-0.275 * * * \\
(0.080)\end{array}$ & $\begin{array}{l}-0.552 * * * \\
(0.074)\end{array}$ & $\begin{array}{l}-0.537 * * * \\
(0.086)\end{array}$ & $\begin{array}{l}-0.537 * * * \\
(0.087)\end{array}$ & $\begin{array}{l}-0.569 * * * \\
(0.078)\end{array}$ \\
\hline$($ delta s)* & log distance & & & & $\begin{array}{l}0.112^{* * *} \\
(0.026)\end{array}$ & $\begin{array}{l}0.183^{* * *} \\
(0.028)\end{array}$ & $\begin{array}{l}0.307 \text { *** } \\
(0.027)\end{array}$ & $\begin{array}{l}0.3233^{* * *} \\
(0.032)\end{array}$ & $\begin{array}{l}0.322 * * * \\
(0.032)\end{array}$ & $\begin{array}{l}0.303 * * * \\
(0.025)\end{array}$ \\
\hline$($ delta s)* & $\log [$ RGDP(importer)/RGDP(exporter)] & & & & & $\begin{array}{l}0.088^{* * *} \\
(0.022)\end{array}$ & $\begin{array}{l}0.184 * * * \\
(0.028)\end{array}$ & $\begin{array}{r}-0.020 \\
(0.076)\end{array}$ & $\begin{array}{r}-0.027 \\
(0.081)\end{array}$ & $\begin{array}{l}0.165^{* * *} \\
(0.036)\end{array}$ \\
\hline$($ delta s)* & log real wage $(\$)$ & & & & & & $\begin{array}{l}-0.070^{* * *} \\
(0.025)\end{array}$ & $\begin{array}{l}-0.0977^{* * *} \\
(0.019)\end{array}$ & $\begin{array}{l}-0.098 * * * \\
(0.019)\end{array}$ & $\begin{array}{l}-0.070 * * * \\
(0.025)\end{array}$ \\
\hline$($ delta s)* & long term inflation & & & & & & & $\begin{array}{r}0.097 \\
(0.073)\end{array}$ & $\begin{array}{r}0.102 \\
(0.077)\end{array}$ & $\begin{array}{r}0.032 \\
(0.039)\end{array}$ \\
\hline$($ delta s)* & long term exchange rate variability & & & & & & & $\begin{array}{r}0.000 \\
(0.000)\end{array}$ & $\begin{array}{r}0.000 \\
(0.000)\end{array}$ & \\
\hline$($ delta s)* & US Importer dummy & & & & & & & & $\begin{array}{r}0.343 \\
(0.288)\end{array}$ & $\begin{array}{r}-0.247 \\
(0.296)\end{array}$ \\
\hline & Error Correction term (ECM) & $\begin{array}{r}0.078 \\
(0.189)\end{array}$ & $\begin{array}{r}0.117 \\
(0.121)\end{array}$ & $\begin{array}{r}0.189 \\
(0.147)\end{array}$ & $\begin{array}{r}0.143 \\
(0.220)\end{array}$ & $\begin{array}{r}0.166 \\
(0.237)\end{array}$ & $\begin{array}{r}0.188 \\
(0.288)\end{array}$ & $\begin{array}{r}0.030 \\
(0.285)\end{array}$ & $\begin{array}{r}0.019 \\
(0.284)\end{array}$ & $\begin{array}{r}0.151 \\
(0.299)\end{array}$ \\
\hline $\mathrm{ECM}$ & trend & & $\begin{array}{l}-0.017 \text { * } \\
(0.009)\end{array}$ & $\begin{array}{l}-0.023 \text { ** } \\
(0.011)\end{array}$ & $\begin{array}{r}-0.002 \\
(0.006)\end{array}$ & $\begin{array}{r}-0.003 \\
(0.006)\end{array}$ & $\begin{array}{r}-0.002 \\
(0.006)\end{array}$ & $\begin{array}{r}0.000 \\
(0.006)\end{array}$ & $\begin{array}{r}0.000 \\
(0.006)\end{array}$ & $\begin{array}{r}-0.003 \\
(0.007)\end{array}$ \\
\hline ECM & $\begin{array}{l}\log [\text { per capitaRGDP(importer) / } \\
\text { per capitaRGDP(exporter)] }\end{array}$ & & & $\begin{array}{r}0.027 \\
(0.025)\end{array}$ & $\begin{array}{r}-0.014 \\
(0.010)\end{array}$ & $\begin{array}{r}-0.018 \\
(0.026)\end{array}$ & $\begin{array}{r}-0.005 \\
(0.038)\end{array}$ & $\begin{array}{r}-0.014 \\
(0.043)\end{array}$ & $\begin{array}{r}-0.011 \\
(0.045)\end{array}$ & $\begin{array}{r}0.001 \\
(0.040)\end{array}$ \\
\hline $\mathrm{ECM}$ & tariff levels & & & & $\begin{array}{r}0.017 \\
(0.035)\end{array}$ & $\begin{array}{r}0.018 \\
(0.036)\end{array}$ & $\begin{array}{r}-0.019 \\
(0.054)\end{array}$ & $\begin{array}{r}0.009 \\
(0.046)\end{array}$ & $\begin{array}{r}0.012 \\
(0.048)\end{array}$ & $\begin{array}{r}-0.011 \\
(0.058)\end{array}$ \\
\hline $\mathrm{ECM}$ & log distance & & & & $\begin{array}{r}-0.034 \\
(0.028)\end{array}$ & $\begin{array}{r}-0.032 \\
(0.028)\end{array}$ & $\begin{array}{r}-0.025 \\
(0.037)\end{array}$ & $\begin{array}{r}-0.014 \\
(0.036)\end{array}$ & $\begin{array}{r}-0.015 \\
(0.037)\end{array}$ & $\begin{array}{r}-0.023 \\
(0.039)\end{array}$ \\
\hline $\mathrm{ECM}$ & $\log [$ RGDP(importer)/RGDP(exporter)] & & & & & $\begin{array}{r}0.008 \\
(0.024)\end{array}$ & $\begin{array}{r}-0.007 \\
(0.039)\end{array}$ & $\begin{array}{r}0.004 \\
(0.039)\end{array}$ & $\begin{array}{r}0.000 \\
(0.043)\end{array}$ & $\begin{array}{r}-0.012 \\
(0.044)\end{array}$ \\
\hline $\mathrm{ECM}$ & log real wage $(\$)$ & & & & & & $\begin{array}{r}0.005 \\
(0.017)\end{array}$ & $\begin{array}{r}0.010 \\
(0.016)\end{array}$ & $\begin{array}{r}0.011 \\
(0.016)\end{array}$ & $\begin{array}{r}0.007 \\
(0.016)\end{array}$ \\
\hline $\mathrm{ECM}$ & long term inflation & & & & & & & $\begin{array}{r}-0.045 \\
(0.074)\end{array}$ & $\begin{array}{r}-0.044 \\
(0.074)\end{array}$ & $\begin{array}{r}-0.032 \\
(0.079)\end{array}$ \\
\hline $\mathrm{ECM}$ & long term exchange rate variability & & & & & & & $\begin{array}{l}0.774 \text { ** } \\
(0.349)\end{array}$ & $\begin{array}{l}0.784 \text { ** } \\
(0.355)\end{array}$ & \\
\hline $\mathrm{ECM}$ & US Importer dummy & & & & & & & & $\begin{array}{r}0.128 \\
(0.175)\end{array}$ & $\begin{array}{r}0.146 \\
(0.206)\end{array}$ \\
\hline & \# of Observations & 677 & 677 & 677 & 568 & 568 & 387 & 376 & 376 & 387 \\
\hline & Adjusted R-squared & -0.025 & 0.747 & 0.765 & 0.942 & 0.947 & 0.953 & 0.952 & 0.952 & 0.952 \\
\hline & Country dummies & yes & yes & yes & yes & yes & yes & yes & yes & yes \\
\hline & Product dummies & yes & yes & yes & yes & yes & yes & yes & yes & yes \\
\hline
\end{tabular}


Table b4: Pass-through to Local competitor's price of Gilbey's Gin

\begin{tabular}{|c|c|c|c|c|c|c|c|c|c|c|}
\hline change in & exchange rate & $\begin{array}{c}\text { Eqn 1 } \\
0.998^{* * *} \\
(0.016)\end{array}$ & $\begin{array}{c}\text { Eqn2 } \\
0.992 \\
(0.028)\end{array}$ & $\begin{array}{c}\text { Eqn3 } \\
0.948^{* * *} \\
(0.038)\end{array}$ & $\begin{array}{c}\text { Eqn } 4 \\
1.348 \\
(0.328)\end{array}$ & $\begin{array}{c}\text { Eqn 5 } \\
1.327 \text { *** } \\
(0.320)\end{array}$ & $\begin{array}{c}\text { Eqn 6 } \\
4.293 * * \\
(1.767)\end{array}$ & $\begin{array}{c}\text { Eqn } 7 \\
5.2588^{* * *} \\
(1.209)\end{array}$ & $\begin{array}{c}\text { Eqn 8 } \\
5.2588^{* * *} \\
(1.209)\end{array}$ & $\begin{array}{c}\text { Eqn 9 } \\
3.838 \text { *** } \\
(1.330)\end{array}$ \\
\hline change in & exporter's price & $\begin{array}{l}0.4777^{* * *} \\
(0.090)\end{array}$ & $\begin{array}{l}0.393^{* * * *} \\
(0.091)\end{array}$ & $\begin{array}{l}0.3644^{* * *} \\
(0.099)^{2}\end{array}$ & $\begin{array}{l}0.389^{* * *} \\
(0.115)^{2}\end{array}$ & $\begin{array}{l}0.385^{* * *} \\
(0.112)^{*}\end{array}$ & $\begin{array}{r}0.204 \\
(0.139)\end{array}$ & $\begin{array}{l}0.232 \text { ** } \\
(0.118)\end{array}$ & $\begin{array}{l}0.232 \text { ** } \\
(0.118)\end{array}$ & $\begin{array}{r}0.177 \\
(0.137)\end{array}$ \\
\hline$($ delta s)* & trend & & $\begin{array}{r}0.001 \\
(0.004)\end{array}$ & $\begin{array}{r}-0.004 \\
(0.010)\end{array}$ & $\begin{array}{r}-0.002 \\
(0.020)\end{array}$ & $\begin{array}{r}-0.009 \\
(0.019)\end{array}$ & $\begin{array}{l}-0.040 * * \\
(0.017)\end{array}$ & $\begin{array}{l}-0.047 * \\
(0.025)\end{array}$ & $\begin{array}{l}-0.047 * \\
(0.025)\end{array}$ & $\begin{array}{l}-0.035 * * \\
(0.015)\end{array}$ \\
\hline$($ delta s)* & $\begin{array}{l}\log [\text { per capitaRGDP(importer) / } \\
\text { per capitaRGDP(exporter)] }\end{array}$ & & & $\begin{array}{r}-0.051 \\
(0.062)\end{array}$ & $\begin{array}{r}-0.062 \\
(0.099)\end{array}$ & $\begin{array}{r}-0.044 \\
(0.095)\end{array}$ & $\begin{array}{l}-0.339 * * * \\
(0.100)\end{array}$ & $\begin{array}{l}-0.536 * * * \\
(0.091)\end{array}$ & $\begin{array}{l}-0.536 * * * \\
(0.091)\end{array}$ & $\begin{array}{l}-0.2811^{* * *} \\
(0.100)\end{array}$ \\
\hline$($ delta s)* & tariff levels & & & & $\begin{array}{r}0.009 \\
(0.036)\end{array}$ & $\begin{array}{r}-0.024 \\
(0.039)\end{array}$ & $\begin{array}{r}0.023 \\
(0.043)\end{array}$ & $\begin{array}{r}0.083 \\
(0.076)\end{array}$ & $\begin{array}{r}0.083 \\
(0.076)\end{array}$ & $\begin{array}{r}-0.081 \\
(0.054)\end{array}$ \\
\hline$($ delta s)* & log distance & & & & $\begin{array}{l}-0.049 * * \\
(0.023)\end{array}$ & $\begin{array}{r}-0.025 \\
(0.030)\end{array}$ & $\begin{array}{l}-0.352 * \\
(0.192)\end{array}$ & $\begin{array}{l}-0.526 * * * \\
(0.133)\end{array}$ & $\begin{array}{l}-0.526 * * * \\
(0.133)\end{array}$ & $\begin{array}{l}-0.272 * \\
(0.146)\end{array}$ \\
\hline$($ delta s)* & $\log [$ RGDP(importer)/RGDP(exporter)] & & & & & $\begin{array}{r}0.027 \\
(0.020)\end{array}$ & $\begin{array}{l}0.1188^{* * *} \\
(0.039)\end{array}$ & $\begin{array}{r}0.022 \\
(0.071)\end{array}$ & $\begin{array}{r}0.022 \\
(0.071)\end{array}$ & $\begin{array}{l}0.1522^{* * *} \\
(0.031)\end{array}$ \\
\hline$($ delta s)* & log real wage $(\$)$ & & & & & & $\begin{array}{l}-0.034 * * * \\
(0.009)\end{array}$ & $\begin{array}{l}-0.026^{* * *} \\
(0.005)\end{array}$ & $\begin{array}{l}-0.026^{* * *} \\
(0.005)\end{array}$ & $\begin{array}{l}-0.035^{* * *} \\
(0.007)\end{array}$ \\
\hline$($ delta s)* & long term inflation & & & & & & & $\begin{array}{l}0.149 * * \\
(0.060)\end{array}$ & $\begin{array}{l}0.149 * * \\
(0.060)\end{array}$ & $\begin{array}{r}0.062 \\
(0.039)\end{array}$ \\
\hline$($ delta s)* & long term exchange rate variability & & & & & & & $\begin{array}{l}-4.7344^{* * *} \\
(1.155)\end{array}$ & $\begin{array}{l}-4.734 * * * \\
(1.155)\end{array}$ & \\
\hline$($ delta s)* & US Importer dummy & & & & & & & & & \\
\hline & Error Correction term (ECM) & $\begin{array}{r}-0.044 \\
(0.033)\end{array}$ & $\begin{array}{r}0.000 \\
(0.036)\end{array}$ & $\begin{array}{r}0.039 \\
(0.053)\end{array}$ & $\begin{array}{r}-0.067 \\
(0.464)\end{array}$ & $\begin{array}{r}-0.094 \\
(0.462)\end{array}$ & $\begin{array}{r}-0.340 \\
(0.659)\end{array}$ & $\begin{array}{l}-1.276 * * \\
(0.645)\end{array}$ & $\begin{array}{l}-1.276 * * \\
(0.645)\end{array}$ & $\begin{array}{r}-0.773 \\
(0.642)\end{array}$ \\
\hline $\mathrm{ECM}$ & trend & & $\begin{array}{l}-0.010 * * * \\
(0.004)\end{array}$ & $\begin{array}{l}-0.010 * * * \\
(0.004)\end{array}$ & $\begin{array}{r}-0.006 \\
(0.004)\end{array}$ & $\begin{array}{r}-0.005 \\
(0.004)\end{array}$ & $\begin{array}{r}-0.003 \\
(0.006)\end{array}$ & $\begin{array}{r}0.001 \\
(0.005)\end{array}$ & $\begin{array}{r}0.001 \\
(0.005)\end{array}$ & $\begin{array}{r}0.000 \\
(0.006)\end{array}$ \\
\hline $\mathrm{ECM}$ & $\begin{array}{l}\log [\text { per capitaRGDP(importer) / } \\
\text { per capitaRGDP(exporter)] }\end{array}$ & & & $\begin{array}{r}0.027 \\
(0.025)\end{array}$ & $\begin{array}{c}0.078 * \\
(0.043)\end{array}$ & $\begin{array}{r}0.063 \\
(0.057)\end{array}$ & $\begin{array}{r}0.082 \\
(0.107)\end{array}$ & $\begin{array}{l}0.2222^{* *} \\
(0.105)\end{array}$ & $\begin{array}{l}0.222 * * \\
(0.105)\end{array}$ & $\begin{array}{l}0.190 * \\
(0.107)\end{array}$ \\
\hline $\mathrm{ECM}$ & tariff levels & & & & $\begin{array}{r}0.021 \\
(0.032)\end{array}$ & $\begin{array}{r}0.011 \\
(0.035)\end{array}$ & $\begin{array}{r}0.003 \\
(0.039)\end{array}$ & $\begin{array}{r}0.018 \\
(0.032)\end{array}$ & $\begin{array}{r}0.018 \\
(0.032)\end{array}$ & $\begin{array}{r}-0.005 \\
(0.038)\end{array}$ \\
\hline $\mathrm{ECM}$ & log distance & & & & $\begin{array}{r}0.010 \\
(0.054)\end{array}$ & $\begin{array}{r}0.016 \\
(0.052)\end{array}$ & $\begin{array}{r}0.023 \\
(0.066)\end{array}$ & $\begin{array}{l}0.123 * \\
(0.068)\end{array}$ & $\begin{array}{c}0.123 * \\
(0.068)\end{array}$ & $\begin{array}{r}0.088 \\
(0.064)\end{array}$ \\
\hline $\mathrm{ECM}$ & $\log [$ RGDP(importer)/RGDP(exporter)] & & & & & $\begin{array}{r}0.003 \\
(0.037)\end{array}$ & $\begin{array}{r}-0.010 \\
(0.061)\end{array}$ & $\begin{array}{r}-0.048 \\
(0.051)\end{array}$ & $\begin{array}{r}-0.048 \\
(0.051)\end{array}$ & $\begin{array}{r}-0.001 \\
(0.060)\end{array}$ \\
\hline $\mathrm{ECM}$ & log real wage $(\$)$ & & & & & & $\begin{array}{l}0.031 * \\
(0.017)\end{array}$ & $\begin{array}{r}0.015 \\
(0.012)\end{array}$ & $\begin{array}{r}0.015 \\
(0.012)\end{array}$ & $\begin{array}{r}0.020 \\
(0.013)\end{array}$ \\
\hline $\mathrm{ECM}$ & long term inflation & & & & & & & $\begin{array}{l}0.2811^{* * * *} \\
(0.066)\end{array}$ & $\begin{array}{l}0.281 * * * \\
(0.066)\end{array}$ & $\begin{array}{l}0.191 \text { ** } \\
(0.088)\end{array}$ \\
\hline $\mathrm{ECM}$ & long term exchange rate variability & & & & & & & $\begin{array}{l}1.381 \text { ** } \\
(0.629)\end{array}$ & $\begin{array}{l}1.381 * * \\
(0.629)\end{array}$ & \\
\hline \multicolumn{11}{|c|}{ ECM* US Importer dummy } \\
\hline & \# of Observations & 723 & 723 & 723 & 577 & 577 & 383 & 374 & 374 & 383 \\
\hline & Adjusted R-squared & 0.946 & 0.946 & 0.947 & 0.950 & 0.950 & 0.960 & 0.943 & 0.943 & 0.961 \\
\hline & Country dummies & yes & yes & yes & yes & yes & yes & yes & yes & yes \\
\hline & Product dummies & yes & yes & yes & yes & yes & yes & yes & yes & yes \\
\hline
\end{tabular}


Table b5: Pass-through to Local competitor's price of Time magazine

\begin{tabular}{|c|c|c|c|c|c|c|c|c|c|c|}
\hline change in & exchange rate & $\begin{array}{c}\text { Eqn 1 } \\
0.972 * * * \\
(0.012)\end{array}$ & $\begin{array}{c}\text { Eqn2 } \\
0.980 \\
(0.022)\end{array}$ & $\begin{array}{c}\text { Eqn3 } \\
0.995 \text { *** } \\
(0.053)\end{array}$ & $\begin{array}{c}\text { Eqn } 4 \\
1.583^{* * *} \\
(0.448)\end{array}$ & $\begin{array}{c}\text { Eqn 5 } \\
1.5788^{* * *} \\
(0.464)\end{array}$ & $\begin{array}{c}\text { Eqn 6 } \\
3.6377^{*} \\
(1.970)\end{array}$ & $\begin{array}{c}\text { Eqn } 7 \\
4.193 \text { ** } \\
(1.662)\end{array}$ & $\begin{array}{c}\text { Eqn 8 } \\
4.193 \\
(1.662)\end{array}$ & $\begin{array}{c}\text { Eqn 9 } \\
3.1844^{*} \\
(1.906)\end{array}$ \\
\hline change in & exporter's price & $\begin{array}{r}0.019 \\
(0.104)\end{array}$ & $\begin{array}{r}0.027 \\
(0.104)\end{array}$ & $\begin{array}{r}0.027 \\
(0.104)\end{array}$ & $\begin{array}{r}-0.050 \\
(0.117)\end{array}$ & $\begin{array}{r}-0.048 \\
(0.117)\end{array}$ & $\begin{array}{r}0.000 \\
(0.140)\end{array}$ & $\begin{array}{r}-0.007 \\
(0.132)\end{array}$ & $\begin{array}{r}-0.007 \\
(0.132)\end{array}$ & $\begin{array}{r}0.012 \\
(0.135)\end{array}$ \\
\hline$($ delta s)* & trend & & $\begin{array}{r}-0.001 \\
(0.004)\end{array}$ & $\begin{array}{r}0.001 \\
(0.008)\end{array}$ & $\begin{array}{r}-0.006 \\
(0.011)\end{array}$ & $\begin{array}{r}-0.004 \\
(0.013)\end{array}$ & $\begin{array}{l}-0.071 * * * \\
(0.021)\end{array}$ & $\begin{array}{l}-0.071 * * * \\
(0.021)\end{array}$ & $\begin{array}{l}-0.071^{* * *} \\
(0.021)^{2}\end{array}$ & $\begin{array}{l}-0.061 * * \\
(0.024)\end{array}$ \\
\hline$($ delta s)* & $\begin{array}{l}\log [\text { per capitaRGDP(importer) / } \\
\text { per capitaRGDP(exporter)] }\end{array}$ & & & $\begin{array}{r}0.017 \\
(0.059)\end{array}$ & $\begin{array}{r}0.017 \\
(0.090)\end{array}$ & $\begin{array}{r}0.018 \\
(0.090)\end{array}$ & $\begin{array}{r}-0.063 \\
(0.128)\end{array}$ & $\begin{array}{l}-0.353^{* * *} \\
(0.103)^{*}\end{array}$ & $\begin{array}{l}-0.353^{* * *} \\
(0.103)^{*}\end{array}$ & $\begin{array}{r}-0.044 \\
(0.129)\end{array}$ \\
\hline$($ delta s)* & tariff levels & & & & $\begin{array}{r}-0.037 \\
(0.033)\end{array}$ & $\begin{array}{r}-0.037 \\
(0.035)\end{array}$ & $\begin{array}{l}-0.175^{* * *} \\
(0.053)\end{array}$ & $\begin{array}{l}-0.440 * * * \\
(0.093)\end{array}$ & $\begin{array}{l}-0.440 * * * \\
(0.093)\end{array}$ & $\begin{array}{l}-0.206^{* *} \\
(0.094)\end{array}$ \\
\hline$($ delta s)* & $\log$ distance & & & & $\begin{array}{r}-0.055 \\
(0.046)\end{array}$ & $\begin{array}{r}-0.052 \\
(0.049)\end{array}$ & $\begin{array}{r}-0.152 \\
(0.210)\end{array}$ & $\begin{array}{r}-0.298 \\
(0.190)\end{array}$ & $\begin{array}{r}-0.298 \\
(0.190)\end{array}$ & $\begin{array}{r}-0.115 \\
(0.207)\end{array}$ \\
\hline$($ delta s)* & $\log [$ RGDP(importer)/RGDP(exporter)] & & & & & $\begin{array}{r}0.007 \\
(0.015)\end{array}$ & $\begin{array}{l}0.211^{* * *} \\
(0.071)\end{array}$ & $\begin{array}{r}0.048 \\
(0.078)\end{array}$ & $\begin{array}{r}0.048 \\
(0.078)\end{array}$ & $\begin{array}{l}0.179^{* *} \\
(0.084)\end{array}$ \\
\hline$($ delta s)* & log real wage (\$) & & & & & & $\begin{array}{l}-0.029 * * * \\
(0.010)\end{array}$ & $\begin{array}{l}-0.013 \text { ** } \\
(0.006)\end{array}$ & $\begin{array}{l}-0.013 \text { ** } \\
(0.006)\end{array}$ & $\begin{array}{l}-0.027 * * * \\
(0.010)\end{array}$ \\
\hline$($ delta s)* & long term inflation & & & & & & & $\begin{array}{l}0.1311^{*} \\
(0.071)\end{array}$ & $\begin{array}{c}0.131 * \\
(0.071)\end{array}$ & $\begin{array}{r}0.042 \\
(0.103)\end{array}$ \\
\hline$($ delta s)* & long term exchange rate variability & & & & & & & $\begin{array}{r}0.900 \\
(0.579)\end{array}$ & $\begin{array}{r}0.900 \\
(0.579)\end{array}$ & \\
\hline$($ delta s)* & US Importer dummy & & & & & & & & & \\
\hline & Error Correction term (ECM) & $\begin{array}{r}-0.059 \\
(0.052)\end{array}$ & $\begin{array}{r}-0.098 \\
(0.096)\end{array}$ & $\begin{array}{r}-0.120 \\
(0.101)\end{array}$ & $\begin{array}{r}0.192 \\
(0.873)\end{array}$ & $\begin{array}{r}-0.030 \\
(0.765)\end{array}$ & $\begin{array}{r}-1.821 \\
(1.265)\end{array}$ & $\begin{array}{r}-1.894 \\
(1.173)\end{array}$ & $\begin{array}{r}-1.894 \\
(1.173)\end{array}$ & $\begin{array}{r}-1.942 \\
(1.260)\end{array}$ \\
\hline $\mathrm{ECM}^{*}$ & trend & & $\begin{array}{r}0.005 \\
(0.008)\end{array}$ & $\begin{array}{r}0.004 \\
(0.008)\end{array}$ & $\begin{array}{r}0.008 \\
(0.010)\end{array}$ & $\begin{array}{r}0.009 \\
(0.011)\end{array}$ & $\begin{array}{r}-0.001 \\
(0.016)\end{array}$ & $\begin{array}{r}-0.004 \\
(0.017)\end{array}$ & $\begin{array}{r}-0.004 \\
(0.017)\end{array}$ & $\begin{array}{r}0.001 \\
(0.016)\end{array}$ \\
\hline $\mathrm{ECM}^{*}$ & $\begin{array}{l}\log [\text { per capitaRGDP(importer) / } \\
\text { per capitaRGDP(exporter)] }\end{array}$ & & & $\begin{array}{r}-0.014 \\
(0.042)\end{array}$ & $\begin{array}{r}-0.015 \\
(0.045)\end{array}$ & $\begin{array}{r}0.003 \\
(0.058)\end{array}$ & $\begin{array}{r}0.066 \\
(0.097)\end{array}$ & $\begin{array}{r}0.094 \\
(0.107)\end{array}$ & $\begin{array}{r}0.094 \\
(0.107)\end{array}$ & $\begin{array}{r}0.091 \\
(0.117)\end{array}$ \\
\hline $\mathrm{ECM}^{*}$ & tariff levels & & & & $\begin{array}{r}-0.001 \\
(0.059)\end{array}$ & $\begin{array}{r}0.002 \\
(0.058)\end{array}$ & $\begin{array}{r}0.137 \\
(0.084)\end{array}$ & $\begin{array}{r}0.086 \\
(0.076)\end{array}$ & $\begin{array}{r}0.086 \\
(0.076)\end{array}$ & $\begin{array}{r}0.137 \\
(0.084)\end{array}$ \\
\hline $\mathrm{ECM}^{*}$ & log distance & & & & $\begin{array}{r}-0.038 \\
(0.106)\end{array}$ & $\begin{array}{r}-0.024 \\
(0.092)\end{array}$ & $\begin{array}{r}0.165 \\
(0.156)\end{array}$ & $\begin{array}{r}0.210 \\
(0.151)\end{array}$ & $\begin{array}{r}0.210 \\
(0.151)\end{array}$ & $\begin{array}{r}0.179 \\
(0.157)\end{array}$ \\
\hline $\mathrm{ECM}^{*}$ & $\log [$ RGDP(importer)/RGDP(exporter)] & & & & & $\begin{array}{r}-0.027 \\
(0.051)\end{array}$ & $\begin{array}{r}-0.026 \\
(0.071)\end{array}$ & $\begin{array}{r}0.024 \\
(0.072)\end{array}$ & $\begin{array}{r}0.024 \\
(0.072)\end{array}$ & $\begin{array}{r}-0.025 \\
(0.070)\end{array}$ \\
\hline $\mathrm{ECM}^{*}$ & log real wage $(\$)$ & & & & & & $\begin{array}{r}0.001 \\
(0.025)\end{array}$ & $\begin{array}{r}0.003 \\
(0.023)\end{array}$ & $\begin{array}{r}0.003 \\
(0.023)\end{array}$ & $\begin{array}{r}-0.001 \\
(0.022)\end{array}$ \\
\hline $\mathrm{ECM}^{*}$ & long term inflation & & & & & & & $\begin{array}{r}-0.102 \\
(0.229)\end{array}$ & $\begin{array}{r}-0.102 \\
(0.229)\end{array}$ & $\begin{array}{r}0.070 \\
(0.294)\end{array}$ \\
\hline $\mathrm{ECM}^{*}$ & long term exchange rate variability & & & & & & & $\begin{array}{r}1.378 \\
(2.056)\end{array}$ & $\begin{array}{r}1.378 \\
(2.056)\end{array}$ & \\
\hline \multicolumn{11}{|c|}{ ECM* US Importer dummy } \\
\hline & \# of Observations & 773 & 773 & 773 & 623 & 623 & 384 & 377 & 377 & 384 \\
\hline & Adjusted R-squared & 0.923 & 0.923 & 0.923 & 0.928 & 0.927 & 0.939 & 0.947 & 0.947 & 0.939 \\
\hline & Country dummies & yes & yes & yes & yes & yes & yes & yes & yes & yes \\
\hline & Product dummies & yes & yes & yes & yes & yes & yes & yes & yes & yes \\
\hline
\end{tabular}


Table b6: Pass-through to Local competitor's price of Kodak film

\begin{tabular}{|c|c|c|c|c|c|c|c|c|c|c|}
\hline change in & exchange rate & $\begin{array}{c}\text { Eqn 1 } \\
0.975^{* * *} \\
(0.010)\end{array}$ & $\begin{array}{c}\text { Eqn2 } \\
0.979 \\
(0.017)\end{array}$ & $\begin{array}{c}\text { Eqn3 } \\
1.016^{* * *} \\
(0.046)\end{array}$ & $\begin{array}{c}\text { Eqn 4 } \\
1.242 \\
(0.205)\end{array}$ & $\begin{array}{c}\text { Eqn 5 } \\
1.242^{* * *} \\
(0.188)\end{array}$ & $\begin{array}{c}\text { Eqn 6 } \\
1.937 * \\
(1.051)\end{array}$ & $\begin{array}{c}\text { Eqn } 7 \\
3.3855^{* * *} \\
(0.884)\end{array}$ & $\begin{array}{c}\text { Eqn 8 } \\
3.385 \\
(0.884)\end{array}$ & $\begin{array}{c}\text { Eqn 9 } \\
1.989 \\
(0.879)\end{array}$ \\
\hline change in & exporter's price & $\begin{array}{l}-0.670 * * * \\
(0.121)\end{array}$ & $\begin{array}{l}-0.636 * * * \\
(0.129)\end{array}$ & $\begin{array}{l}-0.659 * * * \\
(0.131)\end{array}$ & $\begin{array}{l}-0.721 * * * \\
(0.156)\end{array}$ & $\begin{array}{l}-0.714^{* * *} \\
(0.147)\end{array}$ & $\begin{array}{l}-0.523 * * * \\
(0.149)\end{array}$ & $\begin{array}{l}-0.231^{* *} \\
(0.106)\end{array}$ & $\begin{array}{l}-0.2311^{* *} \\
(0.106)\end{array}$ & $\begin{array}{l}-0.499 * * * \\
(0.129)\end{array}$ \\
\hline$($ delta s)* & trend & & $\begin{array}{r}-0.001 \\
(0.003)\end{array}$ & $\begin{array}{r}0.004 \\
(0.007)\end{array}$ & $\begin{array}{r}-0.010 \\
(0.008)\end{array}$ & $\begin{array}{r}-0.006 \\
(0.009)\end{array}$ & $\begin{array}{l}-0.038^{* *} \\
(0.016)\end{array}$ & $\begin{array}{l}-0.039 * * \\
(0.017)\end{array}$ & $\begin{array}{l}-0.039 * * \\
(0.017)\end{array}$ & $\begin{array}{l}-0.038^{* *} \\
(0.018)\end{array}$ \\
\hline$($ delta s)* & $\begin{array}{l}\log [\text { per capitaRGDP(importer) / } \\
\text { per capitaRGDP(exporter)] }\end{array}$ & & & $\begin{array}{r}0.043 \\
(0.057)\end{array}$ & $\begin{array}{r}-0.047 \\
(0.069)\end{array}$ & $\begin{array}{r}-0.041 \\
(0.069)\end{array}$ & $\begin{array}{l}-0.359 * * * \\
(0.091)\end{array}$ & $\begin{array}{l}-0.563 * * * \\
(0.051)\end{array}$ & $\begin{array}{l}-0.563 * * * \\
(0.051)\end{array}$ & $\begin{array}{l}-0.351 * * * \\
(0.082)\end{array}$ \\
\hline$($ delta s)* & tariff levels & & & & $\begin{array}{l}-0.049 * * \\
(0.020)\end{array}$ & $\begin{array}{l}-0.050 * * \\
(0.022)\end{array}$ & $\begin{array}{r}-0.037 \\
(0.041)\end{array}$ & $\begin{array}{l}-0.306 * * * \\
(0.062)\end{array}$ & $\begin{array}{l}-0.306 * * * \\
(0.062)\end{array}$ & $\begin{array}{r}-0.062 \\
(0.060)\end{array}$ \\
\hline$($ delta s)* & $\log$ distance & & & & $\begin{array}{r}-0.020 \\
(0.021)\end{array}$ & $\begin{array}{r}-0.015 \\
(0.021)\end{array}$ & $\begin{array}{r}-0.102 \\
(0.117)\end{array}$ & $\begin{array}{l}-0.303 * * * \\
(0.087)\end{array}$ & $\begin{array}{l}-0.303 * * * \\
(0.087)\end{array}$ & $\begin{array}{r}-0.095 \\
(0.099)\end{array}$ \\
\hline$($ delta s)* & $\log [$ RGDP(importer)/RGDP(exporter)] & & & & & $\begin{array}{r}0.017 \\
(0.012)\end{array}$ & $\begin{array}{r}0.062 \\
(0.048)\end{array}$ & $\begin{array}{r}-0.012 \\
(0.067)\end{array}$ & $\begin{array}{r}-0.012 \\
(0.067)\end{array}$ & $\begin{array}{r}0.086 \\
(0.056)\end{array}$ \\
\hline$($ delta s)* & log real wage $(\$)$ & & & & & & $\begin{array}{l}-0.029 * * * \\
(0.008)\end{array}$ & $\begin{array}{l}-0.014 * * * \\
(0.004)\end{array}$ & $\begin{array}{l}-0.0144^{* * *} \\
(0.004)\end{array}$ & $\begin{array}{l}-0.029 * * * \\
(0.007)\end{array}$ \\
\hline$($ delta s)* & long term inflation & & & & & & & $\begin{array}{l}0.079 * \\
(0.043)\end{array}$ & $\begin{array}{c}0.079 * \\
(0.043)\end{array}$ & $\begin{array}{r}0.006 \\
(0.060)\end{array}$ \\
\hline$($ delta s)* & long term exchange rate variability & & & & & & & $\begin{array}{l}1.757^{* * *} \\
(0.501)^{*}\end{array}$ & $\begin{array}{l}1.757^{* * *} \\
(0.501)^{*}\end{array}$ & \\
\hline$($ delta s)* & US Importer dummy & & & & & & & & & \\
\hline & Error Correction term (ECM) & $\begin{array}{l}-0.048 * \\
(0.027)\end{array}$ & $\begin{array}{l}-0.105 * \\
(0.063)\end{array}$ & $\begin{array}{l}-0.165^{* * *} \\
(0.063)\end{array}$ & $\begin{array}{r}0.004 \\
(0.484)\end{array}$ & $\begin{array}{r}0.288 \\
(0.520)\end{array}$ & $\begin{array}{r}0.183 \\
(0.472)\end{array}$ & $\begin{array}{r}-0.196 \\
(0.421)\end{array}$ & $\begin{array}{r}-0.196 \\
(0.421)\end{array}$ & $\begin{array}{r}0.651 \\
(0.491)\end{array}$ \\
\hline $\mathrm{ECM}^{*}$ & trend & & $\begin{array}{r}0.006 \\
(0.005)\end{array}$ & $\begin{array}{r}0.005 \\
(0.005)\end{array}$ & $\begin{array}{r}0.009 \\
(0.006)\end{array}$ & $\begin{array}{r}0.010 \\
(0.006)\end{array}$ & $\begin{array}{r}0.006 \\
(0.006)\end{array}$ & $\begin{array}{r}-0.002 \\
(0.006)\end{array}$ & $\begin{array}{r}-0.002 \\
(0.006)\end{array}$ & $\begin{array}{r}-0.001 \\
(0.006)\end{array}$ \\
\hline $\mathrm{ECM}^{*}$ & $\begin{array}{l}\log [\text { per capitaRGDP(importer) / } \\
\text { per capitaRGDP(exporter)] }\end{array}$ & & & $\begin{array}{r}-0.051 \\
(0.032)\end{array}$ & $\begin{array}{r}-0.039 \\
(0.034)\end{array}$ & $\begin{array}{r}-0.058 \\
(0.037)\end{array}$ & $\begin{array}{r}-0.023 \\
(0.042)\end{array}$ & $\begin{array}{r}-0.004 \\
(0.043)\end{array}$ & $\begin{array}{r}-0.004 \\
(0.043)\end{array}$ & $\begin{array}{l}-0.081 * \\
(0.045)\end{array}$ \\
\hline $\mathrm{ECM}^{*}$ & tariff levels & & & & $\begin{array}{r}0.055 \\
(0.038)\end{array}$ & $\begin{array}{r}0.049 \\
(0.037)\end{array}$ & $\begin{array}{r}-0.009 \\
(0.035)\end{array}$ & $\begin{array}{r}-0.007 \\
(0.033)\end{array}$ & $\begin{array}{r}-0.007 \\
(0.033)\end{array}$ & $\begin{array}{r}-0.032 \\
(0.034)\end{array}$ \\
\hline $\mathrm{ECM}^{*}$ & log distance & & & & $\begin{array}{r}-0.036 \\
(0.054)\end{array}$ & $\begin{array}{r}-0.058 \\
(0.056)\end{array}$ & $\begin{array}{r}-0.039 \\
(0.058)\end{array}$ & $\begin{array}{r}0.025 \\
(0.051)\end{array}$ & $\begin{array}{r}0.025 \\
(0.051)\end{array}$ & $\begin{array}{r}-0.077 \\
(0.058)\end{array}$ \\
\hline $\mathrm{ECM}^{*}$ & $\log [$ RGDP(importer)/RGDP(exporter)] & & & & & $\begin{array}{r}0.030 \\
(0.025)\end{array}$ & $\begin{array}{r}-0.020 \\
(0.023)\end{array}$ & $\begin{array}{r}0.002 \\
(0.020)\end{array}$ & $\begin{array}{r}0.002 \\
(0.020)\end{array}$ & $\begin{array}{r}-0.008 \\
(0.022)\end{array}$ \\
\hline $\mathrm{ECM}^{*}$ & log real wage $(\$)$ & & & & & & $\begin{array}{r}0.000 \\
(0.012)\end{array}$ & $\begin{array}{r}-0.001 \\
(0.010)\end{array}$ & $\begin{array}{r}-0.001 \\
(0.010)\end{array}$ & $\begin{array}{r}0.001 \\
(0.011)\end{array}$ \\
\hline $\mathrm{ECM}^{*}$ & long term inflation & & & & & & & $\begin{array}{r}-0.034 \\
(0.103)\end{array}$ & $\begin{array}{r}-0.034 \\
(0.103)\end{array}$ & $\begin{array}{l}-0.324 * * * \\
(0.106)\end{array}$ \\
\hline $\mathrm{ECM}^{*}$ & long term exchange rate variability & & & & & & & $\begin{array}{r}-1.055 \\
(0.721)\end{array}$ & $\begin{array}{r}-1.055 \\
(0.721)\end{array}$ & \\
\hline \multicolumn{11}{|c|}{ ECM* US Importer dummy } \\
\hline & \# of Observations & 756 & 756 & 756 & 608 & 608 & 378 & 371 & 371 & 378 \\
\hline & Adjusted R-squared & 0.963 & 0.963 & 0.963 & 0.966 & 0.967 & 0.980 & 0.984 & 0.984 & 0.981 \\
\hline & Country dummies & yes & yes & yes & yes & yes & yes & yes & yes & yes \\
\hline & Product dummies & yes & yes & yes & yes & yes & yes & yes & yes & yes \\
\hline
\end{tabular}


Table b7: Pass-through to Local competitor's price of Cointreau

\begin{tabular}{|c|c|c|c|c|c|c|c|c|c|c|}
\hline change in & exchange rate & $\begin{array}{r}\text { Eqn 1 } \\
0.000 \\
(0.000)\end{array}$ & $\begin{array}{c}\text { Eqn2 } \\
0.000 \\
(0.000)\end{array}$ & $\begin{array}{r}\text { Eqn3 } \\
0.000 \\
(0.000)\end{array}$ & $\begin{array}{r}\text { Eqn 4 } \\
0.000 \\
(0.000)\end{array}$ & $\begin{array}{r}\text { Eqn 5 } \\
0.000 \\
(0.000)\end{array}$ & $\begin{array}{r}\text { Eqn 6 } \\
0.000 \\
(0.000)\end{array}$ & $\begin{array}{r}\text { Eqn } 7 \\
0.000 \\
(0.000)\end{array}$ & $\begin{array}{r}\text { Eqn 8 } \\
0.000 \\
(0.000)\end{array}$ & $\begin{array}{r}\text { Eqn 9 } \\
0.000 \\
(0.000)\end{array}$ \\
\hline change in & exporter's price & $\begin{array}{r}-0.213 \\
(0.719)\end{array}$ & $\begin{array}{l}0.773^{* *} \\
(0.366)\end{array}$ & $\begin{array}{l}0.9066^{* * *} \\
(0.301)\end{array}$ & $\begin{array}{r}0.169 \\
(0.163)\end{array}$ & $\begin{array}{r}-0.019 \\
(0.150)\end{array}$ & $\begin{array}{r}-0.207 \\
(0.171)\end{array}$ & $\begin{array}{r}-0.158 \\
(0.167)\end{array}$ & $\begin{array}{r}-0.162 \\
(0.167)\end{array}$ & $\begin{array}{r}-0.207 \\
(0.169)\end{array}$ \\
\hline$($ delta s)* & trend & & $\begin{array}{l}0.145^{* * *} \\
(0.022)\end{array}$ & $\begin{array}{l}0.170^{* * *} \\
(0.023)^{*}\end{array}$ & $\begin{array}{l}0.031 * \\
(0.018)\end{array}$ & $\begin{array}{r}-0.006 \\
(0.019)\end{array}$ & $\begin{array}{l}-0.084^{* * *} \\
(0.017)\end{array}$ & $\begin{array}{l}-0.077 * * * \\
(0.025)\end{array}$ & $\begin{array}{l}-0.073^{* * *} \\
(0.026)\end{array}$ & $\begin{array}{l}-0.075^{* * *} \\
(0.017)\end{array}$ \\
\hline$($ delta s)* & $\begin{array}{l}\log [\text { per capitaRGDP(importer) / } \\
\text { per capitaRGDP(exporter)] }\end{array}$ & & & $\begin{array}{r}0.061 \\
(0.051)\end{array}$ & $\begin{array}{r}0.012 \\
(0.013)\end{array}$ & $\begin{array}{l}-0.075^{* * *} \\
(0.024)\end{array}$ & $\begin{array}{l}-0.265^{* * *} \\
(0.036)\end{array}$ & $\begin{array}{l}-0.152 * \\
(0.082)\end{array}$ & $\begin{array}{l}-0.154 * \\
(0.084)\end{array}$ & $\begin{array}{l}-0.244^{* * *} \\
(0.037)\end{array}$ \\
\hline$($ delta s)* & tariff levels & & & & $\begin{array}{r}-0.057 \\
(0.089)\end{array}$ & $\begin{array}{l}-0.309 \text { *** } \\
(0.087)\end{array}$ & $\begin{array}{l}-0.6099^{* * *} \\
(0.072)\end{array}$ & $\begin{array}{l}-0.651 * * * \\
(0.084)\end{array}$ & $\begin{array}{l}-0.648^{* * *} \\
(0.084)\end{array}$ & $\begin{array}{l}-0.618^{* * *} \\
(0.069)\end{array}$ \\
\hline$($ delta s)* & $\log$ distance & & & & $\begin{array}{l}0.113^{* * *} \\
(0.028)\end{array}$ & $\begin{array}{l}0.194^{* * *} \\
(0.030)\end{array}$ & $\begin{array}{l}0.325^{* * *} \\
(0.026)\end{array}$ & $\begin{array}{l}0.306 * * * \\
(0.028)\end{array}$ & $\begin{array}{l}0.303^{* * *} \\
(0.028)\end{array}$ & $\begin{array}{l}0.3144^{* * *} \\
(0.024)\end{array}$ \\
\hline$($ delta s)* & $\log [$ RGDP(importer)/RGDP(exporter)] & & & & & $\begin{array}{l}0.098^{* * *} \\
(0.024)\end{array}$ & $\begin{array}{l}0.217^{* * *} \\
(0.028)\end{array}$ & $\begin{array}{r}0.087 \\
(0.073)\end{array}$ & $\begin{array}{r}0.091 \\
(0.077)\end{array}$ & $\begin{array}{l}0.199^{* * *} \\
(0.033)^{*}\end{array}$ \\
\hline$($ delta s)* & log real wage $(\$)$ & & & & & & $\begin{array}{l}-0.066 * * * \\
(0.022)\end{array}$ & $\begin{array}{l}-0.069 * * * \\
(0.019)\end{array}$ & $\begin{array}{l}-0.068^{* * *} \\
(0.019)\end{array}$ & $\begin{array}{l}-0.0622^{* * *} \\
(0.022)\end{array}$ \\
\hline$($ delta s)* & long term inflation & & & & & & & $\begin{array}{l}0.146 * \\
(0.079)\end{array}$ & $\begin{array}{l}0.147 * \\
(0.082)\end{array}$ & $\begin{array}{r}0.043 \\
(0.035)\end{array}$ \\
\hline$($ delta s)* & long term exchange rate variability & & & & & & & $\begin{array}{r}0.000 \\
(0.000)\end{array}$ & $\begin{array}{r}0.000 \\
(0.000)\end{array}$ & \\
\hline$($ delta s)* & US Importer dummy & & & & & & & & $\begin{array}{r}-0.063 \\
(0.350)\end{array}$ & $\begin{array}{r}-0.439 \\
(0.349)\end{array}$ \\
\hline & Error Correction term (ECM) & $\begin{array}{r}0.082 \\
(0.198)\end{array}$ & $\begin{array}{r}-0.027 \\
(0.119)\end{array}$ & $\begin{array}{r}0.199 \\
(0.177)\end{array}$ & $\begin{array}{r}0.135 \\
(0.725)\end{array}$ & $\begin{array}{r}0.155 \\
(0.707)\end{array}$ & $\begin{array}{r}0.059 \\
(0.843)\end{array}$ & $\begin{array}{r}-0.960 \\
(0.775)\end{array}$ & $\begin{array}{r}-0.937 \\
(0.767)\end{array}$ & $\begin{array}{r}0.071 \\
(0.803)\end{array}$ \\
\hline $\mathrm{ECM}$ & trend & & $\begin{array}{l}-0.017 * \\
(0.010)\end{array}$ & $\begin{array}{l}-0.018 * \\
(0.009)\end{array}$ & $\begin{array}{r}-0.003 \\
(0.007)\end{array}$ & $\begin{array}{r}0.000 \\
(0.006)\end{array}$ & $\begin{array}{r}0.005 \\
(0.007)\end{array}$ & $\begin{array}{r}0.005 \\
(0.007)\end{array}$ & $\begin{array}{r}0.006 \\
(0.007)\end{array}$ & $\begin{array}{r}0.003 \\
(0.007)\end{array}$ \\
\hline ECM & $\begin{array}{l}\log [\text { per capitaRGDP(importer) / } \\
\text { per capitaRGDP(exporter)] }\end{array}$ & & & $\begin{array}{c}0.073 * \\
(0.043)\end{array}$ & $\begin{array}{r}-0.005 \\
(0.017)\end{array}$ & $\begin{array}{r}0.031 \\
(0.045)\end{array}$ & $\begin{array}{r}0.065 \\
(0.066)\end{array}$ & $\begin{array}{r}0.101 \\
(0.079)\end{array}$ & $\begin{array}{r}0.127 \\
(0.088)\end{array}$ & $\begin{array}{r}0.036 \\
(0.065)\end{array}$ \\
\hline $\mathrm{ECM}$ & tariff levels & & & & $\begin{array}{r}0.018 \\
(0.072)\end{array}$ & $\begin{array}{r}0.063 \\
(0.068)\end{array}$ & $\begin{array}{r}0.025 \\
(0.083)\end{array}$ & $\begin{array}{r}0.115 \\
(0.083)\end{array}$ & $\begin{array}{r}0.128 \\
(0.085)\end{array}$ & $\begin{array}{r}0.043 \\
(0.084)\end{array}$ \\
\hline $\mathrm{ECM}$ & log distance & & & & $\begin{array}{r}-0.032 \\
(0.084)\end{array}$ & $\begin{array}{r}-0.050 \\
(0.079)\end{array}$ & $\begin{array}{r}-0.030 \\
(0.093)\end{array}$ & $\begin{array}{r}0.060 \\
(0.084)\end{array}$ & $\begin{array}{r}0.050 \\
(0.084)\end{array}$ & $\begin{array}{r}-0.029 \\
(0.092)\end{array}$ \\
\hline $\mathrm{ECM}$ & $\log [$ RGDP(importer)/RGDP(exporter)] & & & & & $\begin{array}{r}-0.038 \\
(0.038)\end{array}$ & $\begin{array}{r}-0.085 \\
(0.060)\end{array}$ & $\begin{array}{r}-0.088 \\
(0.066)\end{array}$ & $\begin{array}{r}-0.122 \\
(0.080)\end{array}$ & $\begin{array}{r}-0.072 \\
(0.061)\end{array}$ \\
\hline $\mathrm{ECM}$ & log real wage $(\$)$ & & & & & & $\begin{array}{r}-0.005 \\
(0.031)\end{array}$ & $\begin{array}{r}0.025 \\
(0.031)\end{array}$ & $\begin{array}{r}0.018 \\
(0.031)\end{array}$ & $\begin{array}{r}-0.012 \\
(0.028)\end{array}$ \\
\hline $\mathrm{ECM}$ & long term inflation & & & & & & & $\begin{array}{l}-0.148 \\
(0.110)\end{array}$ & $\begin{array}{r}-0.120 \\
(0.117)\end{array}$ & $\begin{array}{r}-0.143 \\
(0.106)\end{array}$ \\
\hline $\mathrm{ECM}$ & long term exchange rate variability & & & & & & & $\begin{array}{r}0.320 \\
(0.524)\end{array}$ & $\begin{array}{r}0.253 \\
(0.545)\end{array}$ & \\
\hline $\mathrm{ECM}$ & US Importer dummy & & & & & & & & $\begin{array}{c}0.437 \text { * } \\
(0.266)\end{array}$ & $\begin{array}{c}0.386 * \\
(0.227)\end{array}$ \\
\hline & \# of Observations & 660 & 660 & 660 & 544 & 544 & 373 & 360 & 360 & 373 \\
\hline & Adjusted R-squared & -0.046 & 0.742 & 0.767 & 0.942 & 0.949 & 0.956 & 0.957 & 0.957 & 0.957 \\
\hline & Country dummies & yes & yes & yes & yes & yes & yes & yes & yes & yes \\
\hline & Product dummies & yes & yes & yes & yes & yes & yes & yes & yes & yes \\
\hline
\end{tabular}


Table b8: Pass-through to Local competitor's price of Vermouth

\begin{tabular}{|c|c|c|c|c|c|c|c|c|c|c|}
\hline change in & exchange rate & $\begin{array}{r}\text { Eqn 1 } \\
0.000 \\
(0.000)\end{array}$ & $\begin{array}{r}\text { Eqn2 } \\
0.000 \\
(0.000)\end{array}$ & $\begin{array}{c}\text { Eqn3 } \\
0.000 \\
(0.000)\end{array}$ & $\begin{array}{c}\text { Eqn 4 } \\
0.000 * \\
(0.000)\end{array}$ & $\begin{array}{c}\text { Eqn 5 } \\
0.0000^{* * *} \\
(0.000)\end{array}$ & $\begin{array}{r}\text { Eqn 6 } \\
0.000 \\
(0.000)\end{array}$ & $\begin{array}{r}\text { Eqn } 7 \\
0.000 \\
(0.000)\end{array}$ & $\begin{array}{r}\text { Eqn 8 } \\
0.000 \\
(0.000)\end{array}$ & $\begin{array}{r}\text { Eqn 9 } \\
0.000 \\
(0.000)\end{array}$ \\
\hline change in & exporter's price & $\begin{array}{r}0.239 \\
(0.273)\end{array}$ & $\begin{array}{r}0.155 \\
(0.109)\end{array}$ & $\begin{array}{l}0.174 * * \\
(0.077)\end{array}$ & $\begin{array}{r}0.010 \\
(0.071)\end{array}$ & $\begin{array}{r}-0.012 \\
(0.069)\end{array}$ & $\begin{array}{r}-0.063 \\
(0.084)\end{array}$ & $\begin{array}{r}0.009 \\
(0.070)\end{array}$ & $\begin{array}{r}0.014 \\
(0.071)\end{array}$ & $\begin{array}{r}-0.058 \\
(0.084)\end{array}$ \\
\hline$($ delta s)* & trend & & $\begin{array}{l}0.1433^{* * *} \\
(0.023)^{2}\end{array}$ & $\begin{array}{l}0.100 * * * \\
(0.006)\end{array}$ & $\begin{array}{l}0.035^{* *} \\
(0.016)\end{array}$ & $\begin{array}{r}0.003 \\
(0.018)\end{array}$ & $\begin{array}{l}-0.057 * * * \\
(0.019)\end{array}$ & $\begin{array}{l}-0.054 * \\
(0.030)\end{array}$ & $\begin{array}{l}-0.053 * \\
(0.030)\end{array}$ & $\begin{array}{l}-0.064 * * * \\
(0.020)\end{array}$ \\
\hline$($ delta s)* & $\begin{array}{l}\log [\text { per capitaRGDP(importer) / } \\
\text { per capitaRGDP(exporter)] }\end{array}$ & & & $\begin{array}{l}0.099 * * * \\
(0.009)\end{array}$ & $\begin{array}{r}0.019 \\
(0.014)\end{array}$ & $\begin{array}{l}-0.061 * * \\
(0.026)\end{array}$ & $\begin{array}{l}-0.219 \text { *** } \\
(0.039)\end{array}$ & $\begin{array}{l}-0.165 * * * \\
(0.063)\end{array}$ & $\begin{array}{l}-0.175^{* * *} \\
(0.064)\end{array}$ & $\begin{array}{l}-0.2399^{* * *} \\
(0.042)\end{array}$ \\
\hline$($ delta s)* & tariff levels & & & & $\begin{array}{r}-0.031 \\
(0.075)\end{array}$ & $\begin{array}{l}-0.249 * * * \\
(0.081)\end{array}$ & $\begin{array}{l}-0.576^{* * *} \\
(0.111)\end{array}$ & $\begin{array}{l}-0.678^{* * *} \\
(0.108)\end{array}$ & $\begin{array}{l}-0.676^{* * *} \\
(0.108)\end{array}$ & $\begin{array}{l}-0.574^{* * *} \\
(0.120)\end{array}$ \\
\hline$($ delta s)* & $\log$ distance & & & & $\begin{array}{l}0.092 * * * \\
(0.028)\end{array}$ & $\begin{array}{l}0.158 \text { *** } \\
(0.031)\end{array}$ & $\begin{array}{l}0.3111^{* * *} \\
(0.058)\end{array}$ & $\begin{array}{l}0.3411^{* * *} \\
(0.051)\end{array}$ & $\begin{array}{l}0.3388^{* * *} \\
(0.051)\end{array}$ & $\begin{array}{l}0.320{ }^{* * *} \\
(0.057)\end{array}$ \\
\hline$($ delta s)* & log real wage $(\$)$ & & & & & & $\begin{array}{l}-0.0433^{* *} \\
(0.019)\end{array}$ & $\begin{array}{l}-0.055^{* * *} \\
(0.017)\end{array}$ & $\begin{array}{l}-0.053^{* * *} \\
(0.017)\end{array}$ & $\begin{array}{l}-0.0455^{* *} \\
(0.019)\end{array}$ \\
\hline$($ delta s)* & long term inflation & & & & & & & $\begin{array}{r}0.078 \\
(0.069)\end{array}$ & $\begin{array}{r}0.071 \\
(0.069)\end{array}$ & $\begin{array}{r}-0.028 \\
(0.044)\end{array}$ \\
\hline$($ delta s)* & long term exchange rate variability & & & & & & & $\begin{array}{r}0.000 \\
(0.000)\end{array}$ & $\begin{array}{r}0.000 \\
(0.000)\end{array}$ & \\
\hline$($ delta s)* & US Importer dummy & & & & & & & & $\begin{array}{l}-0.6711^{* *} \\
(0.319)\end{array}$ & $\begin{array}{l}-0.9322^{* * *} \\
(0.321)\end{array}$ \\
\hline & Error Correction term (ECM) & $\begin{array}{r}-0.091 \\
(0.126)\end{array}$ & $\begin{array}{r}-0.031 \\
(0.124)\end{array}$ & $\begin{array}{l}-0.275^{* * *} \\
(0.092)\end{array}$ & $\begin{array}{r}-0.420 \\
(0.286)\end{array}$ & $\begin{array}{r}-0.416 \\
(0.271)\end{array}$ & $\begin{array}{r}-0.516 \\
(0.394)\end{array}$ & $\begin{array}{r}-0.569 \\
(0.413)\end{array}$ & $\begin{array}{r}-0.583 \\
(0.412)\end{array}$ & $\begin{array}{r}-0.419 \\
(0.417)\end{array}$ \\
\hline $\mathrm{ECM}^{*}$ & trend & & $\begin{array}{r}-0.017 \\
(0.012)\end{array}$ & $\begin{array}{r}-0.004 \\
(0.005)\end{array}$ & $\begin{array}{r}-0.003 \\
(0.004)\end{array}$ & $\begin{array}{r}-0.004 \\
(0.004)\end{array}$ & $\begin{array}{r}-0.006 \\
(0.005)\end{array}$ & $\begin{array}{r}-0.004 \\
(0.005)\end{array}$ & $\begin{array}{r}-0.005 \\
(0.005)\end{array}$ & $\begin{array}{r}-0.005 \\
(0.005)\end{array}$ \\
\hline $\mathrm{ECM}^{*}$ & tariff levels & & & & $\begin{array}{r}0.020 \\
(0.027)\end{array}$ & $\begin{array}{r}0.035 \\
(0.026)\end{array}$ & $\begin{array}{l}0.075 * * \\
(0.033)\end{array}$ & $\begin{array}{l}0.082 * * \\
(0.034)\end{array}$ & $\begin{array}{l}0.082 * * \\
(0.034)\end{array}$ & $\begin{array}{l}0.069 \text { ** } \\
(0.034)\end{array}$ \\
\hline $\mathrm{ECM}^{*}$ & log distance & & & & $\begin{array}{r}0.025 \\
(0.032)\end{array}$ & $\begin{array}{r}0.021 \\
(0.030)\end{array}$ & $\begin{array}{r}0.003 \\
(0.040)\end{array}$ & $\begin{array}{r}0.001 \\
(0.042)\end{array}$ & $\begin{array}{r}0.002 \\
(0.042)\end{array}$ & $\begin{array}{r}-0.007 \\
(0.043)\end{array}$ \\
\hline $\mathrm{ECM}^{*}$ & $\log [$ RGDP(importer)/RGDP(exporter)] & & & & & $\begin{array}{r}-0.019 \\
(0.020)\end{array}$ & $\begin{array}{r}0.017 \\
(0.032)\end{array}$ & $\begin{array}{r}0.024 \\
(0.029)\end{array}$ & $\begin{array}{r}0.024 \\
(0.033)\end{array}$ & $\begin{array}{r}0.008 \\
(0.034)\end{array}$ \\
\hline $\mathrm{ECM}^{*}$ & log real wage $(\$)$ & & & & & & $\begin{array}{r}0.029 \\
(0.021)\end{array}$ & $\begin{array}{c}0.036 \\
(0.020)\end{array}$ & $\begin{array}{c}0.037 \text { * } \\
(0.020)\end{array}$ & $\begin{array}{r}0.026 \\
(0.023)\end{array}$ \\
\hline $\mathrm{ECM}^{*}$ & long term inflation & & & & & & & $\begin{array}{r}0.017 \\
(0.040)\end{array}$ & $\begin{array}{r}0.018 \\
(0.041)\end{array}$ & $\begin{array}{r}0.025 \\
(0.046)\end{array}$ \\
\hline $\mathrm{ECM}^{*}$ & long term exchange rate variability & & & & & & & $\begin{array}{r}-0.020 \\
(0.230)\end{array}$ & $\begin{array}{r}-0.059 \\
(0.228)\end{array}$ & \\
\hline $\mathrm{ECM}^{*}$ & US Importer dummy & & & & & & & & $\begin{array}{r}0.040 \\
(0.152)\end{array}$ & $\begin{array}{r}0.065 \\
(0.152)\end{array}$ \\
\hline & \# of Observations & 687 & 687 & 687 & 550 & 550 & 374 & 363 & 363 & 374 \\
\hline & Adjusted R-squared & -0.042 & 0.741 & 0.895 & 0.944 & 0.950 & 0.955 & 0.952 & 0.952 & 0.955 \\
\hline & Country dummies & yes & yes & yes & yes & yes & yes & yes & yes & yes \\
\hline & Product dummies & yes & yes & yes & yes & yes & yes & yes & yes & yes \\
\hline
\end{tabular}


Table c1: Pass-through to Unit Value of Marlboro

\begin{tabular}{|c|c|c|c|c|c|c|c|c|c|c|}
\hline change in & exchange rate & $\begin{array}{c}\text { Eqn 1 } \\
1.036^{* * *} \\
(0.041)\end{array}$ & $\begin{array}{l}\text { Eqn2 } \\
1.093 * * * \\
(0.128)\end{array}$ & $\begin{array}{c}\text { Eqn3 } \\
1.121 * * * \\
(0.215)\end{array}$ & $\begin{array}{c}\text { Eqn 4 } \\
3.102 * * * \\
(0.616)\end{array}$ & $\begin{array}{c}\text { Eqn 5 } \\
3.075^{* * *} \\
(0.796)\end{array}$ & $\begin{array}{r}\text { Eqn 6 } \\
1.213 \\
(2.322)\end{array}$ & $\begin{array}{r}\text { Eqn } 7 \\
2.201 \\
(1.934)\end{array}$ & $\begin{array}{r}\text { Eqn 8 } \\
2.201 \\
(1.934)\end{array}$ & $\begin{array}{r}\text { Eqn 9 } \\
1.021 \\
(2.534)\end{array}$ \\
\hline change in & exporter's price & $\begin{array}{r}0.040 \\
(0.136)\end{array}$ & $\begin{array}{r}0.191 \\
(0.142)\end{array}$ & $\begin{array}{r}0.202 \\
(0.140)\end{array}$ & $\begin{array}{r}0.148 \\
(0.154)\end{array}$ & $\begin{array}{r}0.092 \\
(0.151)\end{array}$ & $\begin{array}{r}0.112 \\
(0.171)\end{array}$ & $\begin{array}{r}0.092 \\
(0.127)\end{array}$ & $\begin{array}{r}0.092 \\
(0.127)\end{array}$ & $\begin{array}{r}0.115 \\
(0.172)\end{array}$ \\
\hline$($ delta s)* & trend & & $\begin{array}{r}-0.007 \\
(0.013)\end{array}$ & $\begin{array}{r}-0.005 \\
(0.019)\end{array}$ & $\begin{array}{r}-0.030 \\
(0.026)\end{array}$ & $\begin{array}{r}-0.007 \\
(0.042)\end{array}$ & $\begin{array}{r}-0.003 \\
(0.054)\end{array}$ & $\begin{array}{r}-0.080 \\
(0.052)\end{array}$ & $\begin{array}{r}-0.080 \\
(0.052)\end{array}$ & $\begin{array}{r}0.017 \\
(0.060)\end{array}$ \\
\hline$($ delta s)* & $\begin{array}{l}\log [\text { per capitaRGDP(importer) / } \\
\text { per capitaRGDP(exporter)] }\end{array}$ & & & $\begin{array}{r}0.027 \\
(0.167)\end{array}$ & $\begin{array}{r}-0.158 \\
(0.222)\end{array}$ & $\begin{array}{r}-0.208 \\
(0.217)\end{array}$ & $\begin{array}{r}0.043 \\
(0.234)\end{array}$ & $\begin{array}{r}0.002 \\
(0.229)\end{array}$ & $\begin{array}{r}0.002 \\
(0.229)\end{array}$ & $\begin{array}{r}-0.019 \\
(0.231)\end{array}$ \\
\hline$($ delta s)* & tariff levels & & & & $\begin{array}{r}-0.204 \\
(0.148)\end{array}$ & $\begin{array}{r}-0.089 \\
(0.191)\end{array}$ & $\begin{array}{r}-0.103 \\
(0.208)\end{array}$ & $\begin{array}{r}-0.250 \\
(0.235)\end{array}$ & $\begin{array}{r}-0.250 \\
(0.235)\end{array}$ & $\begin{array}{r}-0.236 \\
(0.205)\end{array}$ \\
\hline$($ delta s)* & log distance & & & & $\begin{array}{l}-0.197 * * * \\
(0.066)\end{array}$ & $\begin{array}{l}-0.229 * * \\
(0.115)\end{array}$ & $\begin{array}{r}0.033 \\
(0.278)\end{array}$ & $\begin{array}{r}-0.055 \\
(0.173)\end{array}$ & $\begin{array}{r}-0.055 \\
(0.173)\end{array}$ & $\begin{array}{r}0.015 \\
(0.282)\end{array}$ \\
\hline$($ delta s)* & $\log [$ RGDP(importer)/RGDP(exporter)] & & & & & $\begin{array}{r}0.058 \\
(0.066)\end{array}$ & $\begin{array}{r}0.144 \\
(0.136)\end{array}$ & $\begin{array}{r}-0.060 \\
(0.105)\end{array}$ & $\begin{array}{r}-0.060 \\
(0.105)\end{array}$ & $\begin{array}{r}0.046 \\
(0.135)\end{array}$ \\
\hline$($ delta s)* & log real wage $(\$)$ & & & & & & $\begin{array}{r}0.019 \\
(0.015)\end{array}$ & $\begin{array}{r}-0.014 \\
(0.017)\end{array}$ & $\begin{array}{r}-0.014 \\
(0.017)\end{array}$ & $\begin{array}{l}0.036 * \\
(0.019)\end{array}$ \\
\hline$($ delta s)* & long term inflation & & & & & & & $\begin{array}{l}-0.188 * * \\
(0.086)\end{array}$ & $\begin{array}{l}-0.188 * * \\
(0.086)\end{array}$ & $\begin{array}{r}0.025 \\
(0.134)\end{array}$ \\
\hline$($ delta s)* & long term exchange rate variability & & & & & & & $\begin{array}{l}-4.997 * \\
(2.626)\end{array}$ & $\begin{array}{l}-4.997 * \\
(2.626)\end{array}$ & \\
\hline$($ delta s)* & US Importer dummy & & & & & & & & & \\
\hline & Error Correction term (ECM) & $\begin{array}{l}-0.660 * * * \\
(0.079)\end{array}$ & $\begin{array}{l}-0.6177^{* * *} \\
(0.082)\end{array}$ & $\begin{array}{l}-0.464 * * * \\
(0.140)\end{array}$ & $\begin{array}{r}-1.016 \\
(1.184)\end{array}$ & $\begin{array}{r}-0.628 \\
(1.233)\end{array}$ & $\begin{array}{r}-0.790 \\
(1.284)\end{array}$ & $\begin{array}{r}0.302 \\
(0.773)\end{array}$ & $\begin{array}{r}0.302 \\
(0.773)\end{array}$ & $\begin{array}{r}-0.215 \\
(1.197)\end{array}$ \\
\hline ECM* & trend & & $\begin{array}{l}-0.009 * * \\
(0.004)\end{array}$ & $\begin{array}{l}-0.008 * * \\
(0.004)\end{array}$ & $\begin{array}{r}-0.007 \\
(0.005)\end{array}$ & $\begin{array}{r}-0.008 \\
(0.005)\end{array}$ & $\begin{array}{l}-0.010 * * \\
(0.004)\end{array}$ & $\begin{array}{l}-0.008 * * \\
(0.003)\end{array}$ & $\begin{array}{l}-0.008 * * \\
(0.003)\end{array}$ & $\begin{array}{l}-0.013 * * * \\
(0.005)\end{array}$ \\
\hline $\mathrm{ECM}^{*}$ & $\begin{array}{l}\log [\text { per capitaRGDP(importer) / } \\
\text { per capitaRGDP(exporter)] }\end{array}$ & & & $\begin{array}{r}0.107 \\
(0.085)\end{array}$ & $\begin{array}{r}0.134 \\
(0.091)\end{array}$ & $\begin{array}{r}0.093 \\
(0.094)\end{array}$ & $\begin{array}{l}-0.231 * \\
(0.119)\end{array}$ & $\begin{array}{r}-0.158 \\
(0.102)\end{array}$ & $\begin{array}{r}-0.158 \\
(0.102)\end{array}$ & $\begin{array}{l}-0.275 * * \\
(0.119)\end{array}$ \\
\hline $\mathrm{ECM}^{*}$ & tariff levels & & & & $\begin{array}{r}0.006 \\
(0.039)\end{array}$ & $\begin{array}{r}-0.007 \\
(0.038)\end{array}$ & $\begin{array}{r}0.021 \\
(0.045)\end{array}$ & $\begin{array}{r}0.018 \\
(0.034)\end{array}$ & $\begin{array}{r}0.018 \\
(0.034)\end{array}$ & $\begin{array}{r}0.017 \\
(0.046)\end{array}$ \\
\hline $\mathrm{ECM}^{*}$ & $\log$ distance & & & & $\begin{array}{r}0.066 \\
(0.138)\end{array}$ & $\begin{array}{r}0.057 \\
(0.136)\end{array}$ & $\begin{array}{r}-0.007 \\
(0.137)\end{array}$ & $\begin{array}{r}-0.058 \\
(0.090)\end{array}$ & $\begin{array}{r}-0.058 \\
(0.090)\end{array}$ & $\begin{array}{r}-0.061 \\
(0.133)\end{array}$ \\
\hline ECM* & $\log [$ RGDP(importer)/RGDP(exporter)] & & & & & $\begin{array}{r}0.092 \\
(0.057)\end{array}$ & $\begin{array}{r}0.003 \\
(0.075)\end{array}$ & $\begin{array}{l}0.078^{* *} \\
(0.039)\end{array}$ & $\begin{array}{l}0.078^{* *} \\
(0.039)\end{array}$ & $\begin{array}{r}0.047 \\
(0.061)\end{array}$ \\
\hline $\mathrm{ECM}^{*}$ & log real wage $(\$)$ & & & & & & $\begin{array}{r}0.024 \\
(0.019)\end{array}$ & $\begin{array}{r}-0.011 \\
(0.020)\end{array}$ & $\begin{array}{r}-0.011 \\
(0.020)\end{array}$ & $\begin{array}{r}0.044 \\
(0.028)\end{array}$ \\
\hline $\mathrm{ECM}^{*}$ & long term inflation & & & & & & & $\begin{array}{r}0.096 \\
(0.127)\end{array}$ & $\begin{array}{r}0.096 \\
(0.127)\end{array}$ & $\begin{array}{r}-0.183 \\
(0.143)\end{array}$ \\
\hline $\mathrm{ECM}^{*}$ & long term exchange rate variability & & & & & & & $\begin{array}{l}4.599 \text { *** } \\
(0.861)\end{array}$ & $\begin{array}{l}4.599 \text { *** } \\
(0.861)\end{array}$ & \\
\hline
\end{tabular}

ECM* US Importer dummy

\begin{tabular}{|c|c|c|c|c|c|c|c|c|c|}
\hline \# of Observations & 494 & 494 & 494 & 421 & 421 & 307 & 304 & 304 & 307 \\
\hline Adjusted R-squared & 0.727 & 0.730 & 0.732 & 0.755 & 0.758 & 0.664 & 0.789 & 0.789 & 0.673 \\
\hline Country dummies & yes & yes & yes & yes & yes & yes & yes & yes & yes \\
\hline Product dummies & yes & yes & yes & yes & yes & yes & yes & yes & yes \\
\hline
\end{tabular}


Table c2: Pass-through to Unit Value of Coca-Cola

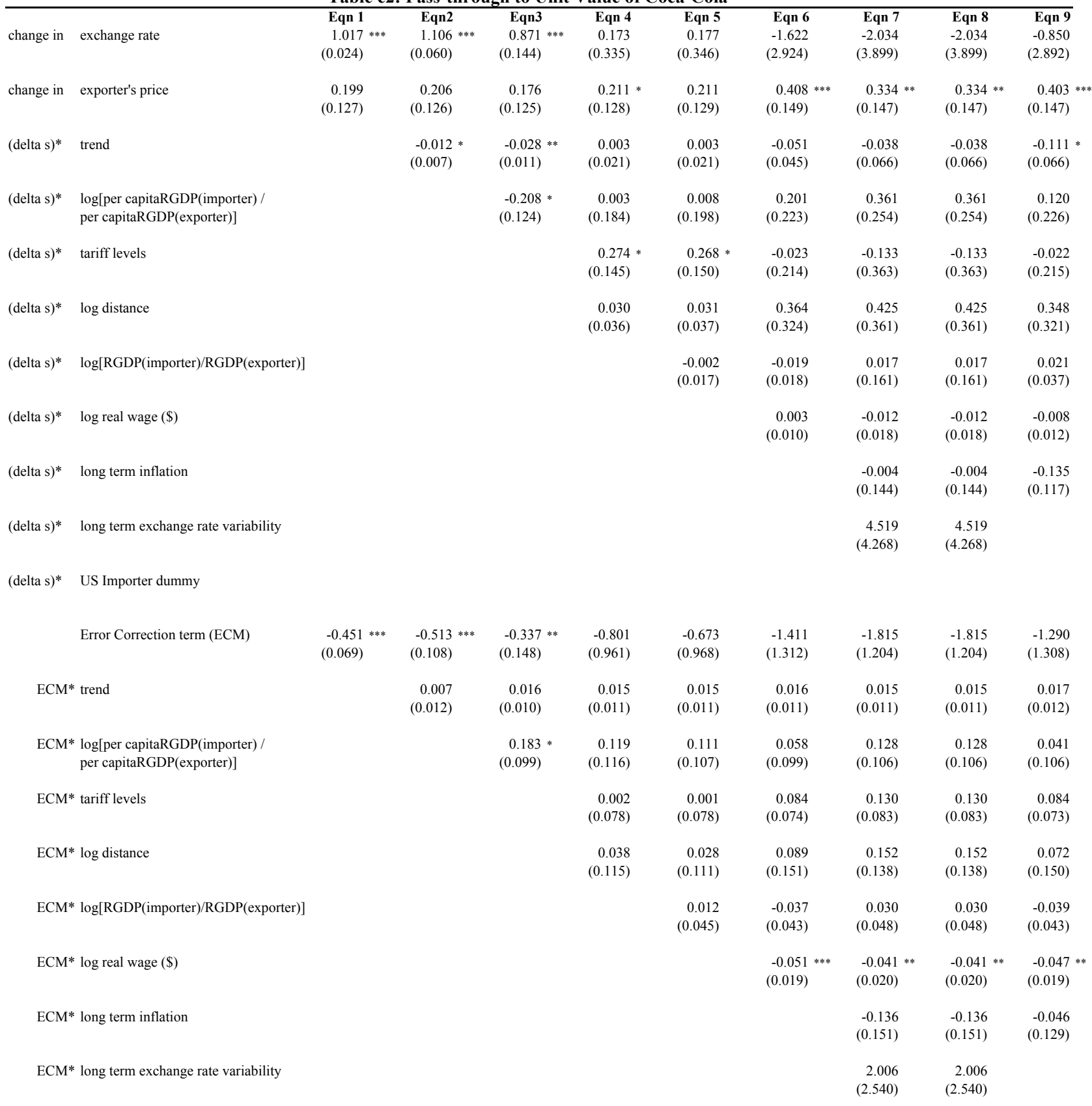

ECM* US Importer dummy

\begin{tabular}{|c|c|c|c|c|c|c|c|c|c|}
\hline \# of Observations & 506 & 506 & 506 & 444 & 444 & 319 & 312 & 312 & 319 \\
\hline Adjusted R-squared & 0.745 & 0.745 & 0.753 & 0.777 & 0.775 & 0.751 & 0.619 & 0.619 & 0.751 \\
\hline Country dummies & yes & yes & yes & yes & yes & yes & yes & yes & yes \\
\hline Product dummies & yes & yes & yes & yes & yes & yes & yes & yes & yes \\
\hline
\end{tabular}


Table c3: Pass-through to Unit Value of Cognac

\begin{tabular}{|c|c|c|c|c|c|c|c|c|c|c|}
\hline change in & exchange rate & $\begin{array}{c}\text { Eqn 1 } \\
0.000 * \\
(0.000)\end{array}$ & $\begin{array}{c}\text { Eqn2 } \\
0.000 * * * \\
(0.000)\end{array}$ & $\begin{array}{c}\text { Eqn3 } \\
0.000 * * * \\
(0.000)\end{array}$ & $\begin{array}{c}\text { Eqn } 4 \\
0.0000^{* *} \\
(0.000)\end{array}$ & $\begin{array}{c}\text { Eqn } 5 \\
0.000 \\
(0.000)\end{array}$ & $\begin{array}{c}\text { Eqn 6 } \\
0.0000^{* * *} \\
(0.000)\end{array}$ & $\begin{array}{c}\text { Eqn } 7 \\
0.000 \\
(0.000)\end{array}$ & $\begin{array}{c}\text { Eqn } 8 \\
0.000^{* * *} \\
(0.000)\end{array}$ & $\begin{array}{c}\text { Eqn 9 } \\
0.000 \\
(0.000)\end{array} * *$ \\
\hline change in & exporter's price & $\begin{array}{r}0.005 \\
(0.274)\end{array}$ & $\begin{array}{l}0.565 \text { *** } \\
(0.141)\end{array}$ & $\begin{array}{l}0.560 \text { *** } \\
(0.140)\end{array}$ & $\begin{array}{l}0.385 \text { *** } \\
(0.124)\end{array}$ & $\begin{array}{l}0.378^{* * *} \\
(0.125)\end{array}$ & $\begin{array}{l}0.493 \text { *** } \\
(0.123)\end{array}$ & $\begin{array}{l}0.350 \text { *** } \\
(0.111)\end{array}$ & $\begin{array}{l}0.342 \text { *** } \\
(0.111)\end{array}$ & $\begin{array}{l}0.419^{* * *} \\
(0.117)\end{array}$ \\
\hline$($ delta s)* & trend & & $\begin{array}{l}0.112^{* * * *} \\
(0.013)\end{array}$ & $\begin{array}{l}0.113^{* * * *} \\
(0.015)\end{array}$ & $\begin{array}{r}-0.002 \\
(0.017)\end{array}$ & $\begin{array}{r}-0.011 \\
(0.021)\end{array}$ & $\begin{array}{r}0.022 \\
(0.023)\end{array}$ & $\begin{array}{c}0.078 * \\
(0.042)\end{array}$ & $\begin{array}{c}0.077 \text { * } \\
(0.042)\end{array}$ & $\begin{array}{l}0.063 * * * \\
(0.023)\end{array}$ \\
\hline$($ delta s)* & tariff levels & & & & $\begin{array}{r}-0.037 \\
(0.143)\end{array}$ & $\begin{array}{r}-0.093 \\
(0.126)\end{array}$ & $\begin{array}{r}-0.071 \\
(0.044)\end{array}$ & $\begin{array}{l}-0.627 \text { *** } \\
(0.094)\end{array}$ & $\begin{array}{l}-0.626^{* * *} \\
(0.093)\end{array}$ & $\begin{array}{l}-0.398^{* * *} \\
(0.078)\end{array}$ \\
\hline$($ delta s)* & $\log$ distance & & & & $\begin{array}{l}0.129 \text { *** } \\
(0.042)\end{array}$ & $\begin{array}{l}0.147^{* * *} \\
(0.041)\end{array}$ & $\begin{array}{l}0.130 \text { *** } \\
(0.027)\end{array}$ & $\begin{array}{l}0.120^{* * *} \\
(0.044)\end{array}$ & $\begin{array}{l}0.122 \text { *** } \\
(0.045)\end{array}$ & $\begin{array}{l}0.129 \text { *** } \\
(0.022)\end{array}$ \\
\hline$($ delta s)* & $\log [$ RGDP(importer)/RGDP(exporter) $]$ & & & & & $\begin{array}{r}-0.035 \\
(0.043)\end{array}$ & $\begin{array}{l}-0.051^{* * *} \\
(0.016)\end{array}$ & $\begin{array}{r}0.061 \\
(0.087)\end{array}$ & $\begin{array}{r}0.077 \\
(0.090)\end{array}$ & $\begin{array}{l}-0.110 \text { *** } \\
(0.021)\end{array}$ \\
\hline$($ delta s)* & log real wage $(\$)$ & & & & & & $\begin{array}{r}0.025 \\
(0.026)\end{array}$ & $\begin{array}{l}0.024 * * \\
(0.012)\end{array}$ & $\begin{array}{l}0.027 \text { ** } \\
(0.013)\end{array}$ & $\begin{array}{r}0.016 \\
(0.020)\end{array}$ \\
\hline$($ delta s)* & long term inflation & & & & & & & $\begin{array}{r}0.142 \\
(0.102)\end{array}$ & $\begin{array}{r}0.129 \\
(0.104)\end{array}$ & $\begin{array}{l}0.235 \text { *** } \\
(0.060)\end{array}$ \\
\hline$($ delta s)* & long term exchange rate variability & & & & & & & $\begin{array}{l}-0.001 * * \\
(0.000)\end{array}$ & $\begin{array}{l}-0.001 \text { ** } \\
(0.000)\end{array}$ & \\
\hline$($ delta s)* & US Importer dummy & & & & & & & & $\begin{array}{l}-0.782 * * \\
(0.322)\end{array}$ & $\begin{array}{l}-0.805 * * * \\
(0.217)\end{array}$ \\
\hline & Error Correction term (ECM) & $\begin{array}{l}-0.414^{* * *} \\
(0.125)\end{array}$ & $\begin{array}{l}-0.457 \text { *** } \\
(0.090)\end{array}$ & $\begin{array}{l}-0.428 \text { *** } \\
(0.084)\end{array}$ & $\begin{array}{l}1.520 \text { *** } \\
(0.478)\end{array}$ & $\begin{array}{l}1.533 \text { *** } \\
(0.482)\end{array}$ & $\begin{array}{c}0.850 * \\
(0.469)\end{array}$ & $\begin{array}{l}0.855 \text { ** } \\
(0.367)\end{array}$ & $\begin{array}{l}0.853 \text { ** } \\
(0.368)\end{array}$ & $\begin{array}{l}1.032 * * \\
(0.440)\end{array}$ \\
\hline $\mathrm{ECM}^{*}$ & trend & & $\begin{array}{l}-0.012 * \\
(0.006)\end{array}$ & $\begin{array}{l}-0.012 \text { ** } \\
(0.006)\end{array}$ & $\begin{array}{l}-0.012 * * * \\
(0.004)\end{array}$ & $\begin{array}{l}-0.012^{* * * *} \\
(0.004)\end{array}$ & $\begin{array}{l}-0.012 * * * \\
(0.004)\end{array}$ & $\begin{array}{r}-0.002 \\
(0.004)\end{array}$ & $\begin{array}{r}-0.001 \\
(0.004)\end{array}$ & $\begin{array}{r}-0.004 \\
(0.005)\end{array}$ \\
\hline $\mathrm{ECM}^{*}$ & tariff levels & & & & $\begin{array}{r}0.035 \\
(0.045)\end{array}$ & $\begin{array}{r}0.030 \\
(0.046)\end{array}$ & $\begin{array}{r}-0.002 \\
(0.057)\end{array}$ & $\begin{array}{r}0.033 \\
(0.049)\end{array}$ & $\begin{array}{r}0.035 \\
(0.049)\end{array}$ & $\begin{array}{r}-0.080 \\
(0.060)\end{array}$ \\
\hline $\mathrm{ECM}^{*}$ & log distance & & & & $\begin{array}{l}-0.2411^{* * *} \\
(0.058)\end{array}$ & $\begin{array}{l}-0.240 * * * \\
(0.058)\end{array}$ & $\begin{array}{l}-0.148^{* * *} \\
(0.056)\end{array}$ & $\begin{array}{l}-0.148 \text { *** } \\
(0.046)\end{array}$ & $\begin{array}{l}-0.149^{* * *} \\
(0.046)\end{array}$ & $\begin{array}{l}-0.161 \text { *** } \\
(0.056)\end{array}$ \\
\hline $\mathrm{ECM}^{*}$ & $\log [$ RGDP(importer)/RGDP(exporter)] & & & & & $\begin{array}{r}0.024 \\
(0.037)\end{array}$ & $\begin{array}{r}-0.048 \\
(0.044)\end{array}$ & $\begin{array}{l}-0.081 * * \\
(0.034)\end{array}$ & $\begin{array}{l}-0.085 * * \\
(0.035)\end{array}$ & $\begin{array}{r}-0.061 \\
(0.045)\end{array}$ \\
\hline $\mathrm{ECM}^{*}$ & log real wage $(\$)$ & & & & & & $\begin{array}{r}-0.019 \\
(0.022)\end{array}$ & $\begin{array}{l}-0.043^{* * *} \\
(0.014)\end{array}$ & $\begin{array}{l}-0.044^{* * *} \\
(0.015)\end{array}$ & $\begin{array}{l}-0.051 \text { *** } \\
(0.018)\end{array}$ \\
\hline $\mathrm{ECM}^{*}$ & long term inflation & & & & & & & $\begin{array}{l}0.1411^{* * *} \\
(0.044)\end{array}$ & $\begin{array}{l}0.142^{* * * *} \\
(0.045)\end{array}$ & $\begin{array}{l}0.177^{* * * *} \\
(0.053)\end{array}$ \\
\hline $\mathrm{ECM}^{*}$ & long term exchange rate variability & & & & & & & $\begin{array}{l}-2.939 \text { *** } \\
(0.515)\end{array}$ & $\begin{array}{l}-2.9388^{* * *} \\
(0.511)\end{array}$ & \\
\hline $\mathrm{ECM}^{*}$ & US Importer dummy & & & & & & & & $\begin{array}{l}0.432 * * \\
(0.190)\end{array}$ & $\begin{array}{l}0.534 \text { ** } \\
(0.209)\end{array}$ \\
\hline & \# of Observations & 542 & 542 & 542 & 470 & 470 & 360 & 353 & 353 & 360 \\
\hline & Adjusted R-squared & 0.011 & 0.739 & 0.738 & 0.871 & 0.871 & 0.880 & 0.875 & 0.875 & 0.892 \\
\hline & Country dummies & yes & yes & yes & yes & yes & yes & yes & yes & yes \\
\hline & Product dummies & yes & yes & yes & yes & yes & yes & yes & yes & yes \\
\hline
\end{tabular}

$*=10 \%, * *=5 \%$, and $* * *=1 \%$ levels of significance

product specific (ps) tariffs 
Table c4: Pass-through to Unit Value of Gilbey's Gin

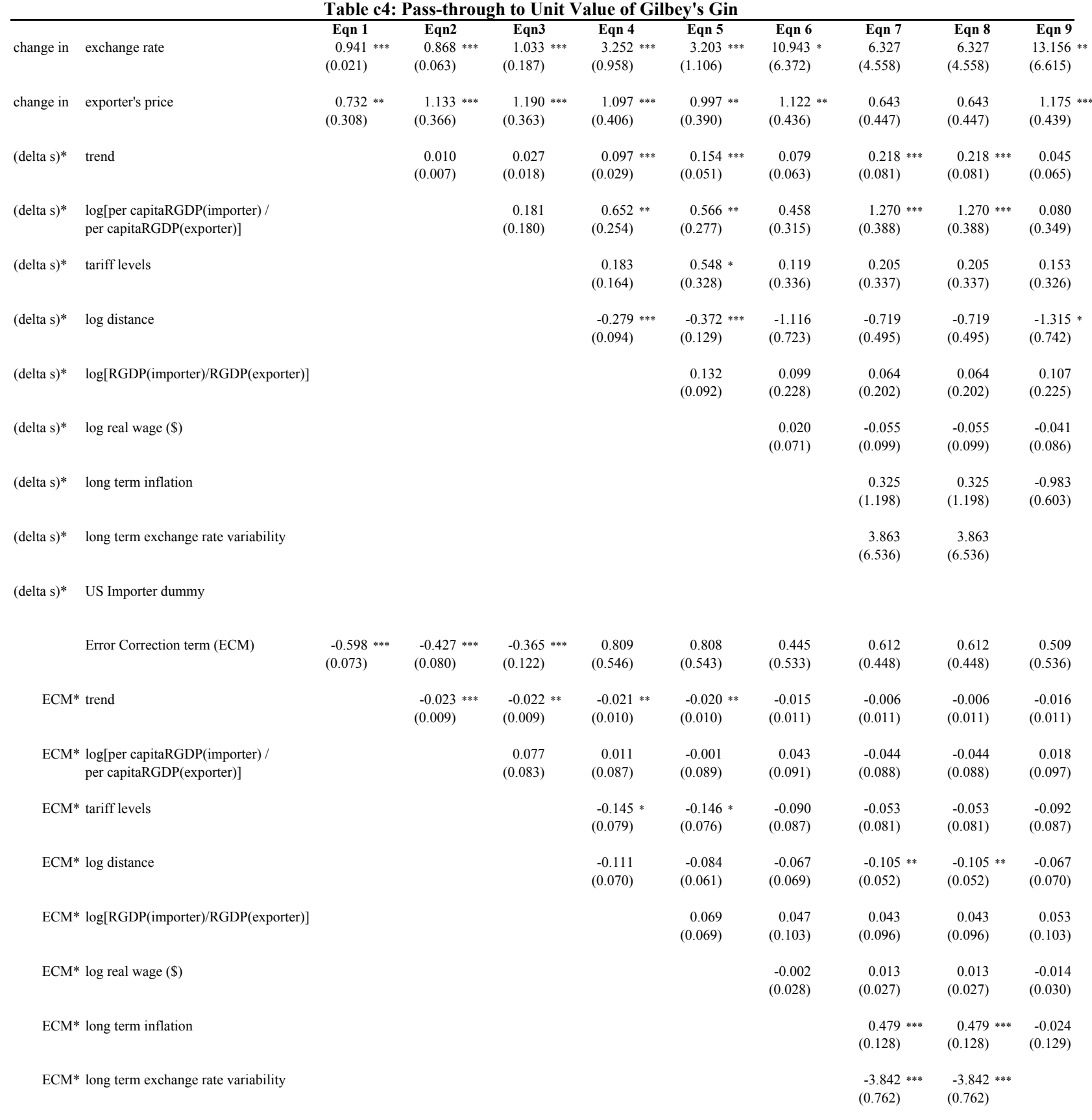

ECM* US Importer dummy

\begin{tabular}{|c|c|c|c|c|c|c|c|c|c|}
\hline \# of Observations & 339 & 339 & 339 & 295 & 295 & 238 & 235 & 235 & 238 \\
\hline Adjusted R-squared & 0.721 & 0.726 & 0.725 & 0.750 & 0.751 & 0.514 & 0.438 & 0.438 & 0.513 \\
\hline Country dummies & yes & yes & yes & yes & yes & yes & yes & yes & yes \\
\hline Product dummies & yes & yes & yes & yes & yes & yes & yes & yes & yes \\
\hline
\end{tabular}


Table c5: Pass-through to Unit Value of Time magazine

\begin{tabular}{|c|c|c|c|c|c|c|c|c|c|c|}
\hline change in & exchange rate & $\begin{array}{c}\text { Eqn 1 } \\
1.013^{* * *} \\
(0.010)\end{array}$ & $\begin{array}{c}\text { Eqn2 } \\
0.9955^{* * *} \\
(0.020)\end{array}$ & $\begin{array}{c}\text { Eqn3 } \\
1.249^{* * *} \\
(0.206)\end{array}$ & $\begin{array}{c}\text { Eqn 4 } \\
1.129 * * \\
(0.470)\end{array}$ & $\begin{array}{c}\text { Eqn 5 } \\
1.1677^{* * *} \\
(0.396)\end{array}$ & $\begin{array}{r}\text { Eqn 6 } \\
-2.222 \\
(4.239)\end{array}$ & $\begin{array}{r}\text { Eqn } 7 \\
3.168 \\
(2.682)\end{array}$ & $\begin{array}{r}\text { Eqn 8 } \\
3.168 \\
(2.682)\end{array}$ & $\begin{array}{r}\text { Eqn 9 } \\
-2.115 \\
(4.035)\end{array}$ \\
\hline change in & exporter's price & $\begin{array}{r}-0.263 \\
(0.289)\end{array}$ & $\begin{array}{r}-0.049 \\
(0.285)\end{array}$ & $\begin{array}{r}-0.077 \\
(0.264)\end{array}$ & $\begin{array}{r}-0.084 \\
(0.292)\end{array}$ & $\begin{array}{r}-0.124 \\
(0.290)\end{array}$ & $\begin{array}{r}0.103 \\
(0.310)\end{array}$ & $\begin{array}{r}0.391 \\
(0.282)\end{array}$ & $\begin{array}{r}0.391 \\
(0.282)\end{array}$ & $\begin{array}{r}0.100 \\
(0.313)\end{array}$ \\
\hline$($ delta s)* & trend & & $\begin{array}{r}0.003 \\
(0.003)\end{array}$ & $\begin{array}{r}0.018 \\
(0.015)\end{array}$ & $\begin{array}{r}0.008 \\
(0.020)\end{array}$ & $\begin{array}{r}0.008 \\
(0.020)\end{array}$ & $\begin{array}{r}0.017 \\
(0.017)\end{array}$ & $\begin{array}{r}-0.028 \\
(0.058)\end{array}$ & $\begin{array}{r}-0.028 \\
(0.058)\end{array}$ & $\begin{array}{r}0.013 \\
(0.027)\end{array}$ \\
\hline$($ delta s)* & $\begin{array}{l}\log [\text { per capitaRGDP(importer) / } \\
\text { per capitaRGDP(exporter)] }\end{array}$ & & & $\begin{array}{r}0.219 \\
(0.183)\end{array}$ & $\begin{array}{r}0.130 \\
(0.220)\end{array}$ & $\begin{array}{r}0.242 \\
(0.265)\end{array}$ & $\begin{array}{r}0.461 \\
(0.312)\end{array}$ & $\begin{array}{r}-0.041 \\
(0.131)\end{array}$ & $\begin{array}{r}-0.041 \\
(0.131)\end{array}$ & $\begin{array}{r}0.453 \\
(0.307)\end{array}$ \\
\hline$($ delta s)* & tariff levels & & & & $\begin{array}{r}0.024 \\
(0.082)\end{array}$ & $\begin{array}{r}0.031 \\
(0.082)\end{array}$ & $\begin{array}{r}0.027 \\
(0.052)\end{array}$ & $\begin{array}{r}0.089 \\
(0.162)\end{array}$ & $\begin{array}{r}0.089 \\
(0.162)\end{array}$ & $\begin{array}{r}0.052 \\
(0.165)\end{array}$ \\
\hline$($ delta s)* & $\log$ distance & & & & $\begin{array}{r}0.001 \\
(0.057)\end{array}$ & $\begin{array}{r}0.002 \\
(0.053)\end{array}$ & $\begin{array}{r}0.399 \\
(0.491)\end{array}$ & $\begin{array}{r}-0.222 \\
(0.256)\end{array}$ & $\begin{array}{r}-0.222 \\
(0.256)\end{array}$ & $\begin{array}{r}0.389 \\
(0.470)\end{array}$ \\
\hline$($ delta s)* & $\log [$ RGDP(importer)/RGDP(exporter) $]$ & & & & & $\begin{array}{l}-0.033^{*} \\
(0.018)\end{array}$ & $\begin{array}{l}-0.060 * \\
(0.034)\end{array}$ & $\begin{array}{r}0.012 \\
(0.176)\end{array}$ & $\begin{array}{r}0.012 \\
(0.176)\end{array}$ & $\begin{array}{r}-0.055 \\
(0.043)\end{array}$ \\
\hline$($ delta s)* & log real wage (\$) & & & & & & $\begin{array}{r}0.009 \\
(0.007)\end{array}$ & $\begin{array}{l}0.015^{*} \\
(0.009)\end{array}$ & $\begin{array}{l}0.015^{*} \\
(0.009)\end{array}$ & $\begin{array}{r}0.008 \\
(0.009)\end{array}$ \\
\hline$($ delta s)* & long term inflation & & & & & & & $\begin{array}{r}0.116 \\
(0.127)\end{array}$ & $\begin{array}{r}0.116 \\
(0.127)\end{array}$ & $\begin{array}{r}-0.018 \\
(0.111)\end{array}$ \\
\hline$($ delta s)* & long term exchange rate variability & & & & & & & $\begin{array}{r}-1.786 \\
(2.570)\end{array}$ & $\begin{array}{r}-1.786 \\
(2.570)\end{array}$ & \\
\hline$($ delta s)* & US Importer dummy & & & & & & & & & \\
\hline & Error Correction term (ECM) & $\begin{array}{l}-0.322 * * * \\
(0.123)\end{array}$ & $\begin{array}{r}-0.139 \\
(0.106)\end{array}$ & $\begin{array}{r}-0.041 \\
(0.102)\end{array}$ & $\begin{array}{r}1.049 \\
(2.601)\end{array}$ & $\begin{array}{r}0.650 \\
(2.598)\end{array}$ & $\begin{array}{r}-4.741 \\
(5.678)\end{array}$ & $\begin{array}{l}-8.961 * * \\
(4.002)\end{array}$ & $\begin{array}{l}-8.961 * * \\
(4.002)\end{array}$ & $\begin{array}{r}-4.807 \\
(6.189)\end{array}$ \\
\hline $\mathrm{ECM}^{*}$ & trend & & $\begin{array}{r}-0.030 \\
(0.019)\end{array}$ & $\begin{array}{r}-0.026 \\
(0.022)\end{array}$ & $\begin{array}{r}-0.018 \\
(0.015)\end{array}$ & $\begin{array}{r}-0.018 \\
(0.015)\end{array}$ & $\begin{array}{r}-0.011 \\
(0.010)\end{array}$ & $\begin{array}{r}0.001 \\
(0.006)\end{array}$ & $\begin{array}{r}0.001 \\
(0.006)\end{array}$ & $\begin{array}{r}-0.011 \\
(0.010)\end{array}$ \\
\hline $\mathrm{ECM}^{*}$ & $\begin{array}{l}\log [\text { per capitaRGDP(importer) / } \\
\text { per capitaRGDP(exporter)] }\end{array}$ & & & $\begin{array}{l}0.198 * \\
(0.113)\end{array}$ & $\begin{array}{r}0.135 \\
(0.097)\end{array}$ & $\begin{array}{r}0.180 \\
(0.138)\end{array}$ & $\begin{array}{r}0.158 \\
(0.253)\end{array}$ & $\begin{array}{l}0.408^{* *} \\
(0.170)\end{array}$ & $\begin{array}{l}0.408 * * \\
(0.170)\end{array}$ & $\begin{array}{r}0.162 \\
(0.278)\end{array}$ \\
\hline $\mathrm{ECM}^{*}$ & tariff levels & & & & $\begin{array}{l}0.4433^{* *} \\
(0.207)\end{array}$ & $\begin{array}{l}0.450 * * \\
(0.208)\end{array}$ & $\begin{array}{l}0.642 \text { ** } \\
(0.323)\end{array}$ & $\begin{array}{r}0.115 \\
(0.110)\end{array}$ & $\begin{array}{r}0.115 \\
(0.110)\end{array}$ & $\begin{array}{l}0.641^{* *} \\
(0.322)\end{array}$ \\
\hline $\mathrm{ECM}^{*}$ & $\log$ distance & & & & $\begin{array}{r}-0.278 \\
(0.349)\end{array}$ & $\begin{array}{r}-0.239 \\
(0.343)\end{array}$ & $\begin{array}{r}0.250 \\
(0.652)\end{array}$ & $\begin{array}{l}0.918 * * \\
(0.447)\end{array}$ & $\begin{array}{l}0.918 * * \\
(0.447)\end{array}$ & $\begin{array}{r}0.258 \\
(0.710)\end{array}$ \\
\hline $\mathrm{ECM}^{*}$ & $\log [$ RGDP(importer)/RGDP(exporter)] & & & & & $\begin{array}{r}-0.028 \\
(0.063)\end{array}$ & $\begin{array}{r}-0.211 \\
(0.162)\end{array}$ & $\begin{array}{r}-0.040 \\
(0.084)\end{array}$ & $\begin{array}{r}-0.040 \\
(0.084)\end{array}$ & $\begin{array}{r}-0.211 \\
(0.163)\end{array}$ \\
\hline $\mathrm{ECM}^{*}$ & log real wage (\$) & & & & & & $\begin{array}{r}0.001 \\
(0.014)\end{array}$ & $\begin{array}{r}0.013 \\
(0.022)\end{array}$ & $\begin{array}{r}0.013 \\
(0.022)\end{array}$ & $\begin{array}{r}0.001 \\
(0.014)\end{array}$ \\
\hline $\mathrm{ECM}^{*}$ & long term inflation & & & & & & & $\begin{array}{r}0.062 \\
(0.187)\end{array}$ & $\begin{array}{r}0.062 \\
(0.187)\end{array}$ & $\begin{array}{r}0.008 \\
(0.096)\end{array}$ \\
\hline $\mathrm{ECM}^{*}$ & long term exchange rate variability & & & & & & & $\begin{array}{l}-4.100^{* * * *} \\
(0.819)\end{array}$ & $\begin{array}{l}-4.100 * * * \\
(0.819)\end{array}$ & \\
\hline
\end{tabular}

ECM* US Importer dummy

\begin{tabular}{|c|c|c|c|c|c|c|c|c|c|}
\hline \# of Observations & 480 & 480 & 480 & 413 & 413 & 299 & 292 & 292 & 299 \\
\hline Adjusted R-squared & 0.724 & 0.732 & 0.744 & 0.812 & 0.812 & 0.827 & 0.863 & 0.863 & 0.826 \\
\hline Country dummies & yes & yes & yes & yes & yes & yes & yes & yes & yes \\
\hline Product dummies & yes & yes & yes & yes & yes & yes & yes & yes & yes \\
\hline
\end{tabular}


Table c6: Pass-through to Unit Value of Kodak film

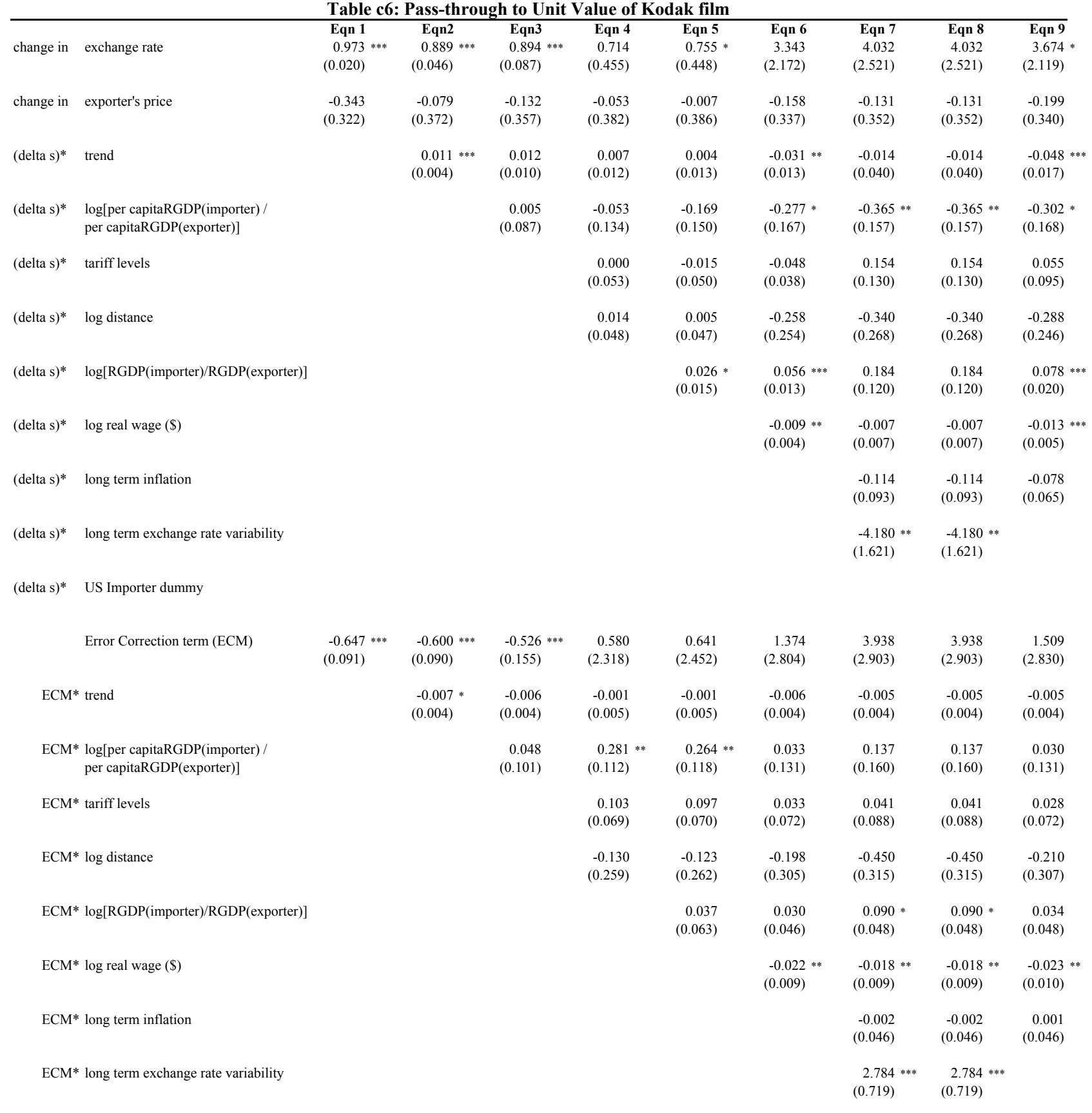

ECM* US Importer dummy

\begin{tabular}{|c|c|c|c|c|c|c|c|c|c|}
\hline \# of Observations & 469 & 469 & 469 & 395 & 395 & 280 & 273 & 273 & 280 \\
\hline Adjusted R-squared & 0.865 & 0.866 & 0.866 & 0.881 & 0.881 & 0.905 & 0.885 & 0.885 & 0.904 \\
\hline Country dummies & yes & yes & yes & yes & yes & yes & yes & yes & yes \\
\hline Product dummies & yes & yes & yes & yes & yes & yes & yes & yes & yes \\
\hline
\end{tabular}


Table c7: Pass-through to Unit Value of Cointreau

\begin{tabular}{|c|c|c|c|c|c|c|c|c|c|c|}
\hline change in & exchange rate & $\begin{array}{c}\text { Eqn 1 } \\
0.000 * \\
(0.000)\end{array}$ & $\begin{array}{c}\text { Eqn2 } \\
0.000 * * * \\
(0.000)\end{array}$ & $\begin{array}{c}\text { Eqn3 } \\
0.000^{* * *} \\
(0.000)\end{array}$ & $\begin{array}{c}\text { Eqn } 4 \\
0.000 \\
(0.000)\end{array}$ & $\begin{array}{c}\text { Eqn 5 } \\
0.000 * * \\
(0.000)\end{array}$ & $\begin{array}{c}\text { Eqn 6 } \\
0.0000^{* * *} \\
(0.000)\end{array}$ & $\begin{array}{c}\text { Eqn } 7 \\
0.000 * * * \\
(0.000)\end{array}$ & $\begin{array}{c}\text { Eqn } 8 \\
0.000^{* * *} \\
(0.000)\end{array}$ & $\begin{array}{c}\text { Eqn 9 } \\
0.000 \\
(0.000)\end{array}$ \\
\hline change in & exporter's price & $\begin{array}{r}0.005 \\
(0.274)\end{array}$ & $\begin{array}{l}0.565 \text { *** } \\
(0.141)\end{array}$ & $\begin{array}{l}0.5600^{* * *} \\
(0.140)\end{array}$ & $\begin{array}{l}0.385 \text { *** } \\
(0.124)\end{array}$ & $\begin{array}{l}0.378^{* * * *} \\
(0.125)\end{array}$ & $\begin{array}{l}0.4933^{* * *} \\
(0.123)\end{array}$ & $\begin{array}{l}0.350 * * * \\
(0.111)\end{array}$ & $\begin{array}{l}0.342^{* * * *} \\
(0.111)\end{array}$ & $\begin{array}{l}0.419 \text { *** } \\
(0.117)\end{array}$ \\
\hline$($ delta s)* & trend & & $\begin{array}{l}0.112^{* * * *} \\
(0.013)\end{array}$ & $\begin{array}{l}0.113^{* * * *} \\
(0.015)\end{array}$ & $\begin{array}{r}-0.002 \\
(0.017)\end{array}$ & $\begin{array}{r}-0.011 \\
(0.021)\end{array}$ & $\begin{array}{r}0.022 \\
(0.023)\end{array}$ & $\begin{array}{c}0.078 * \\
(0.042)\end{array}$ & $\begin{array}{c}0.077 \text { * } \\
(0.042)\end{array}$ & $\begin{array}{l}0.063 * * * \\
(0.023)\end{array}$ \\
\hline$($ delta s)* & tariff levels & & & & $\begin{array}{r}-0.037 \\
(0.143)\end{array}$ & $\begin{array}{r}-0.093 \\
(0.126)\end{array}$ & $\begin{array}{r}-0.071 \\
(0.044)\end{array}$ & $\begin{array}{l}-0.627 \text { *** } \\
(0.094)\end{array}$ & $\begin{array}{l}-0.626^{* * *} \\
(0.093)\end{array}$ & $\begin{array}{l}-0.398^{* * *} \\
(0.078)\end{array}$ \\
\hline$($ delta s)* & log distance & & & & $\begin{array}{l}0.129 * * * \\
(0.042)\end{array}$ & $\begin{array}{l}0.147^{* * *} \\
(0.041)\end{array}$ & $\begin{array}{l}0.130 \text { *** } \\
(0.027)\end{array}$ & $\begin{array}{l}0.120 * * * \\
(0.044)\end{array}$ & $\begin{array}{l}0.122 \text { *** } \\
(0.045)\end{array}$ & $\begin{array}{l}0.129 \text { *** } \\
(0.022)\end{array}$ \\
\hline$($ delta s)* & $\log [$ RGDP(importer)/RGDP(exporter) $]$ & & & & & $\begin{array}{r}-0.035 \\
(0.043)\end{array}$ & $\begin{array}{l}-0.051^{* * *} \\
(0.016)\end{array}$ & $\begin{array}{r}0.061 \\
(0.087)\end{array}$ & $\begin{array}{r}0.077 \\
(0.090)\end{array}$ & $\begin{array}{l}-0.110^{* * *} \\
(0.021)\end{array}$ \\
\hline$($ delta s)* & log real wage (\$) & & & & & & $\begin{array}{r}0.025 \\
(0.026)\end{array}$ & $\begin{array}{l}0.024 * * \\
(0.012)\end{array}$ & $\begin{array}{l}0.027^{* *} \\
(0.013)\end{array}$ & $\begin{array}{r}0.016 \\
(0.020)\end{array}$ \\
\hline$($ delta s)* & long term inflation & & & & & & & $\begin{array}{r}0.142 \\
(0.102)\end{array}$ & $\begin{array}{r}0.129 \\
(0.104)\end{array}$ & $\begin{array}{l}0.235 \text { *** } \\
(0.060)\end{array}$ \\
\hline$($ delta s)* & long term exchange rate variability & & & & & & & $\begin{array}{l}-0.001 * * \\
(0.000)\end{array}$ & $\begin{array}{l}-0.0011^{* *} \\
(0.000)\end{array}$ & \\
\hline$($ delta s)* & US Importer dummy & & & & & & & & $\begin{array}{l}-0.782 * * \\
(0.322)\end{array}$ & $\begin{array}{l}-0.805 \text { *** } \\
(0.217)\end{array}$ \\
\hline & Error Correction term (ECM) & $\begin{array}{l}-0.414^{* * *} \\
(0.125)\end{array}$ & $\begin{array}{l}-0.457 \text { *** } \\
(0.090)\end{array}$ & $\begin{array}{l}-0.428 \text { *** } \\
(0.084)\end{array}$ & $\begin{array}{l}1.520 * * * \\
(0.478)\end{array}$ & $\begin{array}{l}1.5333^{* * * *} \\
(0.482)\end{array}$ & $\begin{array}{c}0.850 * \\
(0.469)\end{array}$ & $\begin{array}{l}0.855 * * \\
(0.367)\end{array}$ & $\begin{array}{l}0.853 \text { ** } \\
(0.368)\end{array}$ & $\begin{array}{l}1.032 \text { ** } \\
(0.440)\end{array}$ \\
\hline $\mathrm{ECM}^{*}$ & trend & & $\begin{array}{l}-0.012 * \\
(0.006)\end{array}$ & $\begin{array}{l}-0.012 \text { ** } \\
(0.006)\end{array}$ & $\begin{array}{l}-0.012 * * * \\
(0.004)\end{array}$ & $\begin{array}{l}-0.012^{* * * *} \\
(0.004)\end{array}$ & $\begin{array}{l}-0.012 \text { *** } \\
(0.004)\end{array}$ & $\begin{array}{r}-0.002 \\
(0.004)\end{array}$ & $\begin{array}{r}-0.001 \\
(0.004)\end{array}$ & $\begin{array}{r}-0.004 \\
(0.005)\end{array}$ \\
\hline $\mathrm{ECM}^{*}$ & tariff levels & & & & $\begin{array}{r}0.035 \\
(0.045)\end{array}$ & $\begin{array}{r}0.030 \\
(0.046)\end{array}$ & $\begin{array}{r}-0.002 \\
(0.057)\end{array}$ & $\begin{array}{r}0.033 \\
(0.049)\end{array}$ & $\begin{array}{r}0.035 \\
(0.049)\end{array}$ & $\begin{array}{r}-0.080 \\
(0.060)\end{array}$ \\
\hline $\mathrm{ECM}^{*}$ & log distance & & & & $\begin{array}{l}-0.2411^{* * *} \\
(0.058)\end{array}$ & $\begin{array}{l}-0.240 * * * \\
(0.058)\end{array}$ & $\begin{array}{l}-0.148^{* * *} \\
(0.056)\end{array}$ & $\begin{array}{l}-0.148 \text { *** } \\
(0.046)\end{array}$ & $\begin{array}{l}-0.149^{* * *} \\
(0.046)\end{array}$ & $\begin{array}{l}-0.161 \text { *** } \\
(0.056)\end{array}$ \\
\hline $\mathrm{ECM}^{*}$ & $\log [$ RGDP(importer)/RGDP(exporter)] & & & & & $\begin{array}{r}0.024 \\
(0.037)\end{array}$ & $\begin{array}{r}-0.048 \\
(0.044)\end{array}$ & $\begin{array}{l}-0.081 * * \\
(0.034)\end{array}$ & $\begin{array}{l}-0.085 * * \\
(0.035)\end{array}$ & $\begin{array}{r}-0.061 \\
(0.045)\end{array}$ \\
\hline $\mathrm{ECM}^{*}$ & log real wage (\$) & & & & & & $\begin{array}{r}-0.019 \\
(0.022)\end{array}$ & $\begin{array}{l}-0.043 * * * \\
(0.014)\end{array}$ & $\begin{array}{l}-0.044^{* * *} \\
(0.015)\end{array}$ & $\begin{array}{l}-0.0511^{* * *} \\
(0.018)\end{array}$ \\
\hline $\mathrm{ECM}^{*}$ & long term inflation & & & & & & & $\begin{array}{l}0.141 \text { *** } \\
(0.044)\end{array}$ & $\begin{array}{l}0.142^{* * * *} \\
(0.045)\end{array}$ & $\begin{array}{l}0.177 \text { **** } \\
(0.053)\end{array}$ \\
\hline $\mathrm{ECM}^{*}$ & long term exchange rate variability & & & & & & & $\begin{array}{l}-2.939 * * * \\
(0.515)\end{array}$ & $\begin{array}{l}-2.9388^{* * *} \\
(0.511)\end{array}$ & \\
\hline $\mathrm{ECM}^{*}$ & US Importer dummy & & & & & & & & $\begin{array}{l}0.432 * * \\
(0.190)\end{array}$ & $\begin{array}{l}0.534 \text { ** } \\
(0.209)\end{array}$ \\
\hline & \# of Observations & 542 & 542 & 542 & 470 & 470 & 360 & 353 & 353 & 360 \\
\hline & Adjusted R-squared & 0.011 & 0.739 & 0.738 & 0.871 & 0.871 & 0.880 & 0.875 & 0.875 & 0.892 \\
\hline & Country dummies & yes & yes & yes & yes & yes & yes & yes & yes & yes \\
\hline & Product dummies & yes & yes & yes & yes & yes & yes & yes & yes & yes \\
\hline
\end{tabular}

$*=10 \%, * *=5 \%$, and $* * *=1 \%$ levels of significance

product specific (ps) tariffs 
Table c8: Pass-through to Unit Value of Vermouth

\begin{tabular}{|c|c|c|c|c|c|c|c|c|c|c|}
\hline change in & exchange rate & $\begin{array}{r}\text { Eqn 1 } \\
0.000 \\
(0.000)\end{array}$ & $\begin{array}{c}\text { Eqn2 } \\
0.000 * * * \\
(0.000)\end{array}$ & $\begin{array}{c}\text { Eqn3 } \\
0.000 \\
(0.000)\end{array}$ & $\begin{array}{c}\text { Eqn } 4 \\
0.000 * * \\
(0.000)\end{array}$ & $\begin{array}{r}\text { Eqn 5 } \\
0.000 \\
(0.000)\end{array}$ & $\begin{array}{r}\text { Eqn 6 } \\
0.000 \\
(0.000)\end{array}$ & $\begin{array}{c}\text { Eqn } 7 \\
0.000 * \\
(0.000)\end{array}$ & $\begin{array}{c}\text { Eqn 8 } \\
0.000 * \\
(0.000)\end{array}$ & $\begin{array}{r}\text { Eqn 9 } \\
0.000 \\
(0.000)\end{array}$ \\
\hline change in & exporter's price & $\begin{array}{r}0.107 \\
(0.528)\end{array}$ & $\begin{array}{l}1.023 * * * \\
(0.232)\end{array}$ & $\begin{array}{l}0.886 \text { *** } \\
(0.200)\end{array}$ & $\begin{array}{l}1.013^{* * * *} \\
(0.204)\end{array}$ & $\begin{array}{l}1.072 * * * \\
(0.207)\end{array}$ & $\begin{array}{l}1.259^{* * * *} \\
(0.204)^{2}\end{array}$ & $\begin{array}{c}0.361 * \\
(0.218)\end{array}$ & $\begin{array}{r}0.355 \\
(0.219)\end{array}$ & $\begin{array}{l}1.228 \text { *** } \\
(0.209)\end{array}$ \\
\hline$($ delta s)* & trend & & $\begin{array}{l}0.125 * * * \\
(0.018)\end{array}$ & $\begin{array}{l}0.065 \text { ** } \\
(0.027)\end{array}$ & $\begin{array}{r}0.006 \\
(0.015)\end{array}$ & $\begin{array}{r}-0.014 \\
(0.021)\end{array}$ & $\begin{array}{l}0.030 * \\
(0.016)\end{array}$ & $\begin{array}{r}-0.050 \\
(0.044)\end{array}$ & $\begin{array}{r}-0.049 \\
(0.045)\end{array}$ & $\begin{array}{c}0.033 * \\
(0.019)\end{array}$ \\
\hline$($ delta s)* & tariff levels & & & & $\begin{array}{r}0.112 \\
(0.116)\end{array}$ & $\begin{array}{r}-0.010 \\
(0.119)\end{array}$ & $\begin{array}{r}-0.046 \\
(0.055)\end{array}$ & $\begin{array}{l}-0.382 * * * \\
(0.109)\end{array}$ & $\begin{array}{l}-0.383^{* * *} \\
(0.110)\end{array}$ & $\begin{array}{r}-0.119 \\
(0.106)\end{array}$ \\
\hline$($ delta s)* & log distance & & & & $\begin{array}{l}0.073 \text { ** } \\
(0.033)\end{array}$ & $\begin{array}{l}0.124 \text { *** } \\
(0.040)\end{array}$ & $\begin{array}{r}0.097 \\
(0.061)\end{array}$ & $\begin{array}{l}0.189 * * * \\
(0.070)\end{array}$ & $\begin{array}{l}0.187 \text { *** } \\
(0.070)\end{array}$ & $\begin{array}{c}0.113 * \\
(0.059)\end{array}$ \\
\hline$($ delta s)* & $\log [$ RGDP(importer)/RGDP(exporter)] & & & & & $\begin{array}{r}-0.075 \\
(0.048)\end{array}$ & $\begin{array}{l}-0.104^{* * *} \\
(0.033)\end{array}$ & $\begin{array}{r}-0.059 \\
(0.079)\end{array}$ & $\begin{array}{r}-0.058 \\
(0.084)\end{array}$ & $\begin{array}{l}-0.122 \text { *** } \\
(0.036)\end{array}$ \\
\hline$($ delta s)* & log real wage $(\$)$ & & & & & & $\begin{array}{r}0.012 \\
(0.032)\end{array}$ & $\begin{array}{l}-0.051 \text { ** } \\
(0.023)\end{array}$ & $\begin{array}{l}-0.051 \text { ** } \\
(0.024)\end{array}$ & $\begin{array}{r}0.005 \\
(0.031)\end{array}$ \\
\hline$($ delta s)* & long term inflation & & & & & & & $\begin{array}{r}0.087 \\
(0.090)\end{array}$ & $\begin{array}{r}0.087 \\
(0.094)\end{array}$ & $\begin{array}{r}0.042 \\
(0.066)\end{array}$ \\
\hline$($ delta s)* & long term exchange rate variability & & & & & & & $\begin{array}{c}0.000 * \\
(0.000)\end{array}$ & $\begin{array}{c}0.000 * \\
(0.000)\end{array}$ & \\
\hline$($ delta s)* & US Importer dummy & & & & & & & & $\begin{array}{r}-0.124 \\
(0.274)\end{array}$ & $\begin{array}{r}-0.121 \\
(0.259)\end{array}$ \\
\hline & Error Correction term (ECM) & $\begin{array}{l}-0.800^{* * *} \\
(0.221)\end{array}$ & $\begin{array}{l}-0.599 * * * \\
(0.173)\end{array}$ & $\begin{array}{l}-0.588^{* * *} \\
(0.174)\end{array}$ & $\begin{array}{r}1.279 \\
(0.784)\end{array}$ & $\begin{array}{r}1.090 \\
(0.739)\end{array}$ & $\begin{array}{r}0.570 \\
(0.738)\end{array}$ & $\begin{array}{r}0.510 \\
(0.748)\end{array}$ & $\begin{array}{r}0.520 \\
(0.748)\end{array}$ & $\begin{array}{r}0.781 \\
(0.743)\end{array}$ \\
\hline $\mathrm{ECM}^{*}$ & trend & & $\begin{array}{l}-0.023 * * \\
(0.009)\end{array}$ & $\begin{array}{l}-0.016 * \\
(0.008)\end{array}$ & $\begin{array}{l}-0.017 \text { ** } \\
(0.007)\end{array}$ & $\begin{array}{l}-0.017 \text { ** } \\
(0.007)\end{array}$ & $\begin{array}{l}-0.020 * * * \\
(0.007)\end{array}$ & $\begin{array}{r}-0.010 \\
(0.006)\end{array}$ & $\begin{array}{r}-0.010 \\
(0.006)\end{array}$ & $\begin{array}{l}-0.016 \text { ** } \\
(0.007)\end{array}$ \\
\hline $\mathrm{ECM}^{*}$ & tariff levels & & & & $\begin{array}{l}-0.249 \text { ** } \\
(0.101)\end{array}$ & $\begin{array}{l}-0.229 * * \\
(0.106)\end{array}$ & $\begin{array}{r}-0.163 \\
(0.107)\end{array}$ & $\begin{array}{r}-0.033 \\
(0.079)\end{array}$ & $\begin{array}{r}-0.031 \\
(0.079)\end{array}$ & $\begin{array}{l}-0.171 \\
(0.104)\end{array}$ \\
\hline $\mathrm{ECM}^{*}$ & log distance & & & & $\begin{array}{l}-0.167 * \\
(0.096)\end{array}$ & $\begin{array}{l}-0.154 * \\
(0.089)\end{array}$ & $\begin{array}{r}-0.032 \\
(0.110)\end{array}$ & $\begin{array}{r}-0.076 \\
(0.086)\end{array}$ & $\begin{array}{r}-0.078 \\
(0.086)\end{array}$ & $\begin{array}{r}-0.050 \\
(0.109)\end{array}$ \\
\hline $\mathrm{ECM}^{*}$ & $\log [$ RGDP(importer)/RGDP(exporter)] & & & & & $\begin{array}{r}-0.046 \\
(0.069)\end{array}$ & $\begin{array}{l}-0.076 \\
(0.072)\end{array}$ & $\begin{array}{r}-0.073 \\
(0.063)\end{array}$ & $\begin{array}{l}-0.076 \\
(0.064)\end{array}$ & $\begin{array}{r}-0.094 \\
(0.072)\end{array}$ \\
\hline $\mathrm{ECM}^{*}$ & log real wage $(\$)$ & & & & & & $\begin{array}{l}-0.058 \text { ** } \\
(0.026)\end{array}$ & $\begin{array}{l}-0.027 \\
(0.029)\end{array}$ & $\begin{array}{l}-0.028 \\
(0.029)\end{array}$ & $\begin{array}{l}-0.077^{* * *} \\
(0.028)\end{array}$ \\
\hline $\mathrm{ECM}^{*}$ & long term inflation & & & & & & & $\begin{array}{r}0.081 \\
(0.084)\end{array}$ & $\begin{array}{r}0.082 \\
(0.084)\end{array}$ & $\begin{array}{l}0.186 * * * \\
(0.071)\end{array}$ \\
\hline $\mathrm{ECM}^{*}$ & long term exchange rate variability & & & & & & & $\begin{array}{l}-7.447^{* * *} \\
(1.029)\end{array}$ & $\begin{array}{l}-7.445 * * * \\
(1.029)\end{array}$ & \\
\hline $\mathrm{ECM}^{*}$ & US Importer dummy & & & & & & & & $\begin{array}{c}0.400 \\
(0.229)\end{array}$ & $\begin{array}{l}0.615 \text { ** } \\
(0.295)\end{array}$ \\
\hline & \# of Observations & 493 & 493 & 493 & 428 & 428 & 331 & 324 & 324 & 331 \\
\hline & Adjusted R-squared & 0.029 & 0.747 & 0.782 & 0.860 & 0.862 & 0.856 & 0.869 & 0.868 & 0.856 \\
\hline & Country dummies & yes & yes & yes & yes & yes & yes & yes & yes & yes \\
\hline & Product dummies & yes & yes & yes & yes & yes & yes & yes & yes & yes \\
\hline
\end{tabular}

Full sample

Country dummie

$*=10 \%, * *=5 \%$, and $* * *=1 \%$ levels of significance

product specific (ps) tariffs 